\section{RECFIVED \\ JAN 301998 \\ OSTI}

Mechanical and Tribological Properties of Ion Beam-Processed Surfaces

\section{Los Alamos}

NATIONAL LABORATORY

Los Alamos National Laboratory is operated by the University of California for the United States Department of Energy under contract W-7405-ENG-36. 


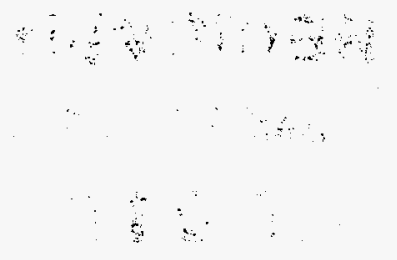

This thesis was accepted by the Department of Materials Engineering, University of Maryland at College Park, College Park, Maryland 20742, in partial fulfillment of the requirements for the degree of Doctor of Philosophy. The text and illustrations are the independent work of the author and only the front matter has been edited by the CIC-1 Writing and Editing Staff to conform with Department of Energy and Los Alamos National Laboratory publication policies.

An Affirmative Action/Equal Opportunity Employer

This report was prepared as an account of work sponsored by an agency of the United States Government. Neither The Regents of the University of California, the United States Government nor any agency thereof, nor any of their employees, makes any warranty, express or implied, or assumes any legal liability or responsibility for the accuracy, completeness, or usefulness of any information, apparatus, product, or process disclosed, or represents that its use would not infringe privately owned rights. Reference herein to any specific commercial product, process, or service by trade name, trademark, manufacturer, or otherwise, does not necessarily constitute or imply its endorsement, recommendation, or favoring by The Regents of the University of California, the United States Government, or any agency thereof. The views and opinions of authors expressed herein do not necessarily state or reflect those of The Regents of the University of California, the United States Government, or any agency thereof. Las Alamos National Laboratory strongly supports academic freedom and a researcher's right to publish; as an institution, however, the Laboratory does not endorse the viewopoint of a publication or guarantee its technical correctness. 
Mechanical and Tribological Properties of

Ion Beam-Processed Surfaces

Padma Kodali*

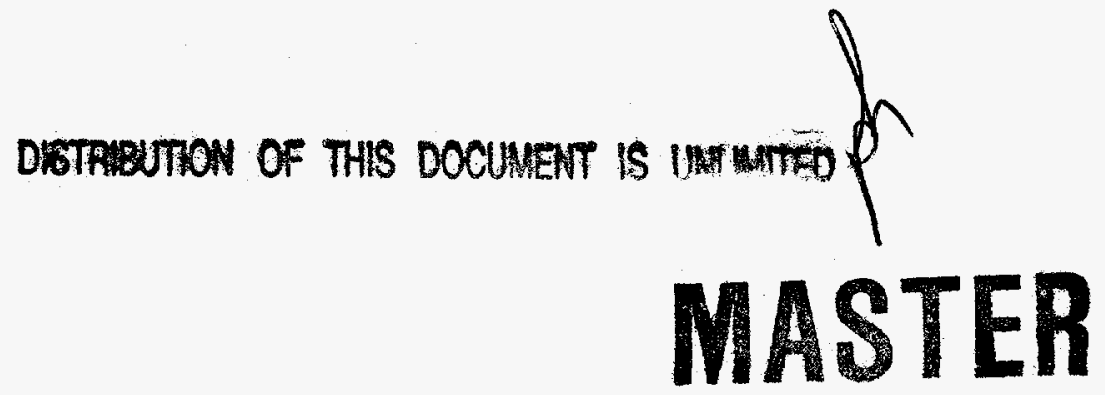

* Graduate Research Assistant at Los Alamos. Currently with Research and Development Facilities of Cummins Engine Company, Inc., Mail Code: CMC-003, 4500 Leeds Avenue, Suite 118, Charleston, SC 29405.

\section{Los Alamos \\ NATIONAL LABORATORY}

Los Alamos, New Mexico 87545 


\section{DISCLAIMER}

Portions of this document may be illegible electronic image products. Images are produced from the best available original document. 


\section{ACKNOWLEDGMENTS}

An experimental thesis at a national laboratory is not completed without help from many people, and this thesis is no exception.

Prof. Aris Christou provided the guidance and encouragement required to complete this thesis. This work would not be in the form of a thesis without his help and support, and I am grateful to him. I am also indebted to Dr. Steve Hsu for his help from time to time in the course of this investigation.

Dr. Michael Nastasi extended excellent supervision, encouragement, and moral support. His thought-provoking discussions and enormous amount of patience helped me not only to complete my thesis, but also to make me an independent researcher. Whenever I approached Mike with a problem, he always had several solutions to a single problem. This influenced my scientific thinking. I cannot find sufficient words to thank Mike, for without his help, this work would not have been possible. I will always carry a profound respect for Mike. Mike also provided the opportunity to interact with several of his collaborators, J.P. Hirvonen and P. Torri of the University of Helsinki, and S. Fayeulle, Laboratoire MMP_URACNRS 447 Ecole Centrale De Lyon, France. I am thankful to all of them for making my research enjoyable.

Dr. Don Parkin and Dr. Mike Stevens at the Center for Materials Science of Los Alamos National Laboratory provided me with the financial assistance to perform this research. Don and Mike have been constant sources of encouragement at all stages of my project and helped the work proceed as smoothly as possible. Despite their busy schedule, they always took time to inquire about my work. They contributed significantly to my professional success. I am deeply indebted to both of them.

Working at the center, I had a unique opportunity to interact with many renowned scientists. I am particularly grateful to Prof. Rishi Raj, University of Colorado, and Prof. David Embury, McMaster University, who took a keen interest in my project and helped in many ways. I would not have been able to finish this work without their guidance. My immense gratitude to David for his recommendations, which were invaluable for the structure of this dissertation.

Most of my work has been performed at the Ion Beam Materials Laboratory (IBML). My sincere and heart felt acknowledgments go to the beam boys:

Dr. Joseph R. Tesmer, Dr. Carl Maggiore, Mark Hollander, Caleb Evans, and Kent Scarborrough. I received immense technical and mechanical training, not only to use the beams, but also to design experimental setups. Joe and Carl made a strong contribution to my professional success.

Acknowledgments also go to Dr. Harriet Kung, Dr. Terrance Mitchell, and Jim Smith, who were always there to help me with technical and operational problems relating to electron microscopes. In addition to Harriet's professional help, she has been a great friend during my stay at LANL.

I am also indebted to Dr. Marilyn Hawley, who always took time out to answer my questions and trained me in AFM. I owe much to Marilyn for her constant encouragement at all stages of my work. 
I am also grateful to Dr. Kevin Walter and Dr. Kris Kern, who always had enough time for my tribology trivia. Kevin's help at many stages of my work cannot be expressed in words. My interactions with Dr. Don Rej, Dr. Blake Wood, Dr. R. Schwarz, Dr. Kevin Hubbard, Dr. T. R. Jervis, Dr. K. Sickafus, and Dr. S. Malloy helped me in the course of my stay at LANL.

I would like to thank my friends Luke Emmert, Chris Heller, Robert Gettings, and Prasad Chaparala for their moral support. Besides being great climbing partners at Los Alamos, Luke and Chris helped me professionally in many ways. I wouldn't have survived graduate school without the great friendship of Rob and Prasad.

Lastly, but most strongly, I acknowledge Dr. Jorn Larsen Basse from NSF, whom I met at the Gordon Conference in Tribology, 1996. His moral support and helpful discussions during the last stages of this dissertation were invaluable. I am grateful to Dr. Steve Shaffer of Battelle Memorial Institute and Prof. S. Ramlingam of the University of Minnesota, whom I met at the Wear of Materials Conference, 1997, for their valuable suggestions and encouragement.

Acknowledgments are not complete with out expressing my gratitude to the Ion Beam guru, Prof. James Mayer, Arizona State University, for his encouragement during his visits to LANL, and to Carol Noones for her untiring help in editing. 


\section{TABLE OF CONTENTS}

LIST OF TABLES

LIST OF FIGURES ...........................................................................................

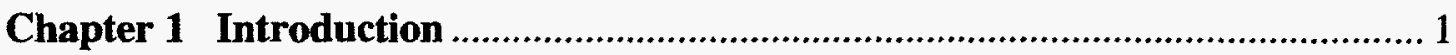

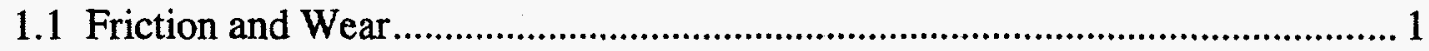

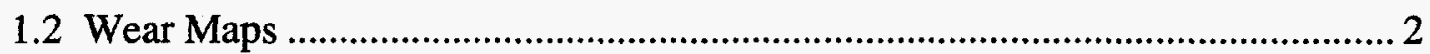

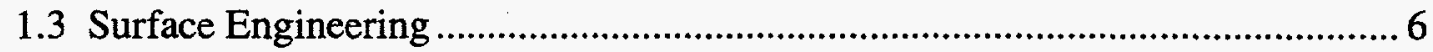

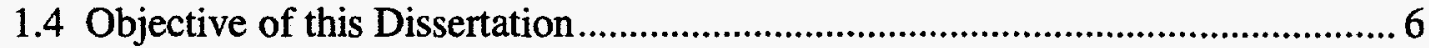

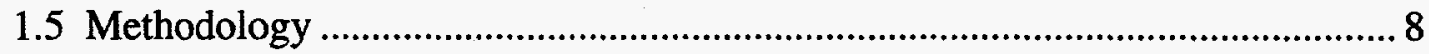

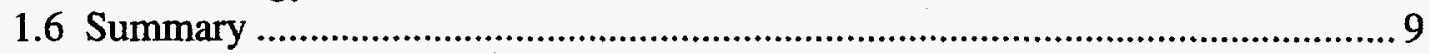

Chapter 2 Overview of Tribology and Surface Engineering ………...................... 10

2.1 Importance of Tribology ............................................................................ 10

2.2 Tribological Contact of Surfaces................................................................ 13

2.3 Theories of Friction and Wear....................................................................... 16

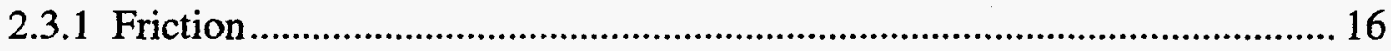

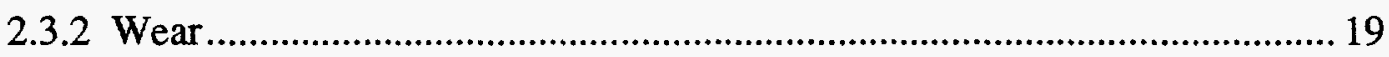

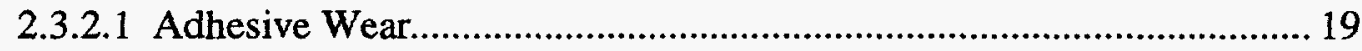

2.3.2.2 Abrasive and Delamination Wear........................................................ 21

2.3.2.3 Role of Fracture Toughness................................................................ 22

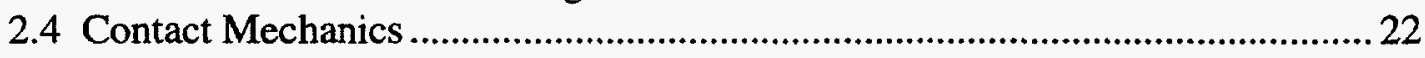

2.4.1 Contact Stress Field Caused by a Spherical Indenter on an Elastic Half-

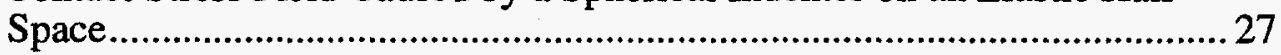

2.4.2 von Mises Yield Criterion...................................................................... 27

2.4.3 Contact Stress Field Caused by a Spherical Indenter on a Layered Elastic Half-Space .............................................................................................. 34

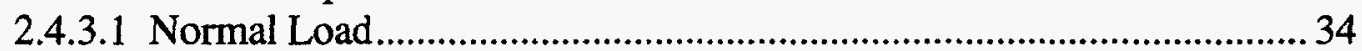

2.4.3.2 Normal Load plus Tangential Load....................................................... 34

2.5 Introduction to Surface Engineering ................................................................... 45

Chapter 3 Experimental Techniques ............................................................ 49

3.1 Surface Engineering Methods ……………........................................................ 49

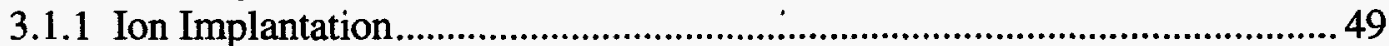

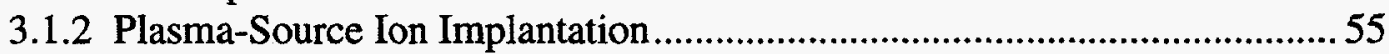

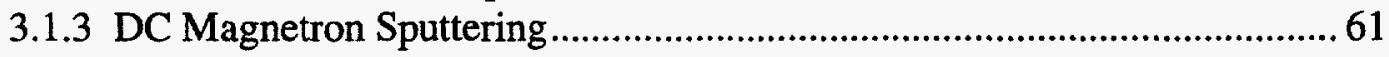

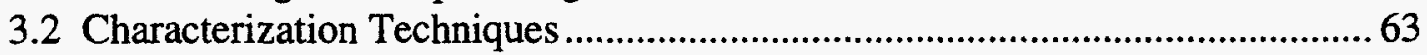

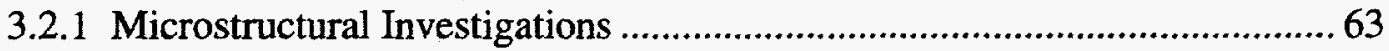

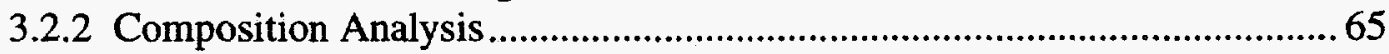

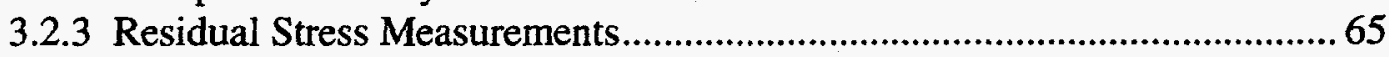

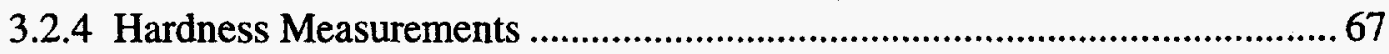

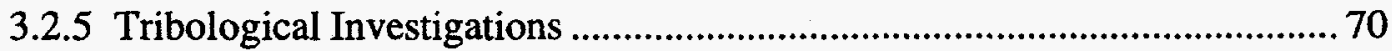

3.2.5.1 Pin-on-Disc Apparatus..................................................................... 70

3.2.5.2 Wear Measurements ...................................................................... 71

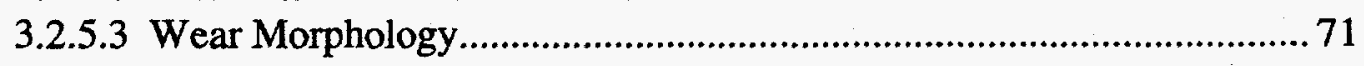


3.2.6 Fracture Toughness Measurements.............................................................. 71

Chapter 4 Implantation Studies of Silicon …………......................................... 76

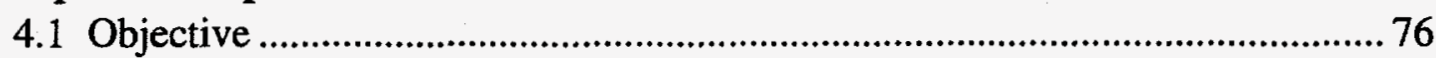

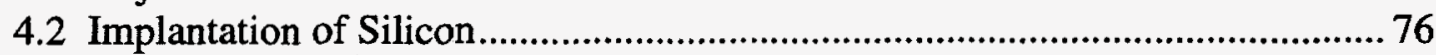

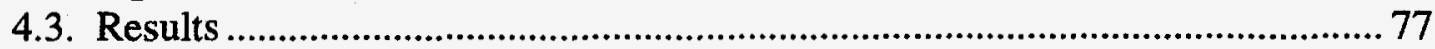

4.3.1. Determination of Projected Ranges and Straggling of the

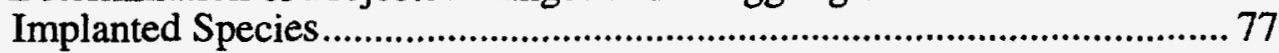

4.3.1.1 Calculations from Analytical Expressions................................................ 77

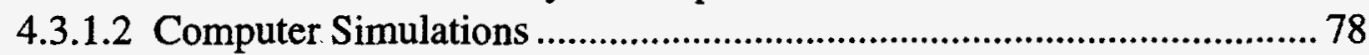

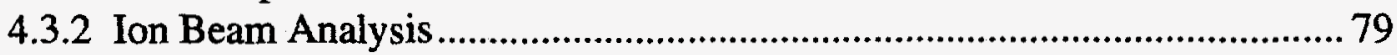

4.3.2.1 Rutherford Backscattering Spectrometry Analysis ................................ 79

4.3.2.2 Channeling Measurements................................................................... 89

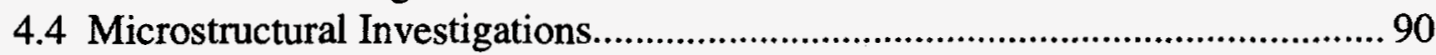

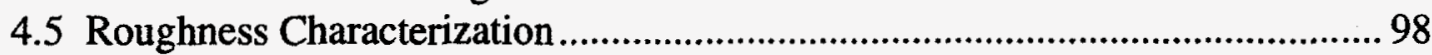

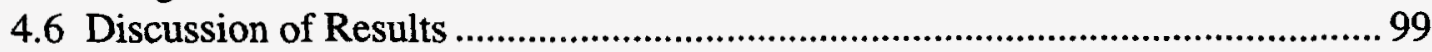

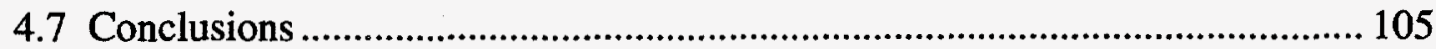

Chapter 5 Mechanical and Tribological Properties of Implanted Silicon........ 107

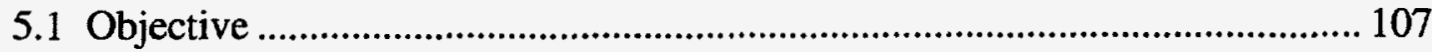

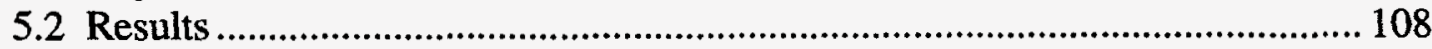

5.2.1 Surface Hardness Measurements ........................................................... 109

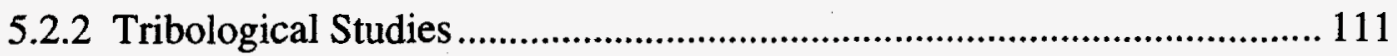

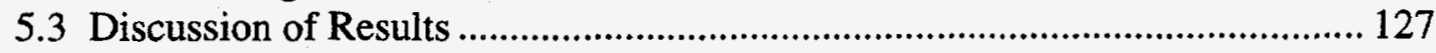

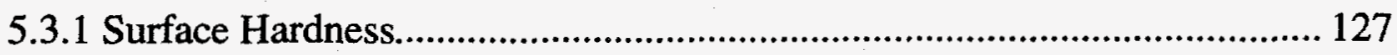

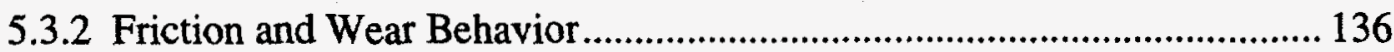

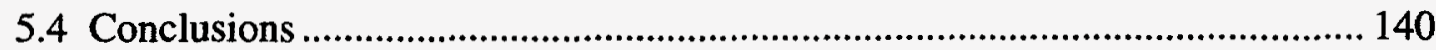

Chapter 6 Investigations of Amorphous Diamond-like Carbon Coatings ........ 142

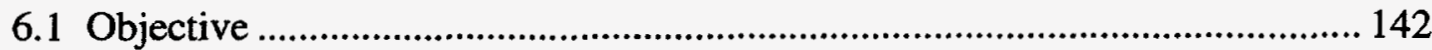

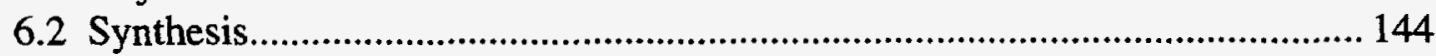

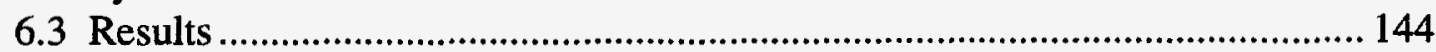

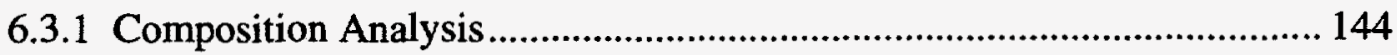

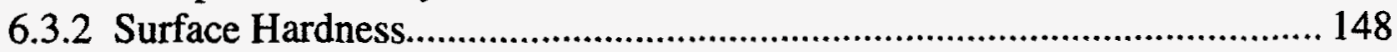

6.3.3 Apparent Fracture Toughness .............................................................. 148

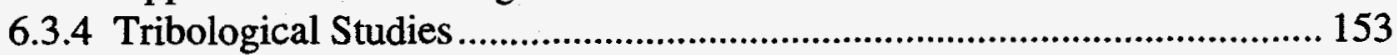

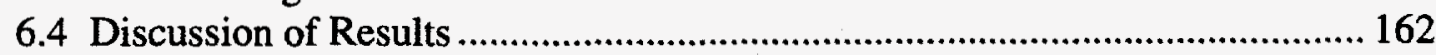

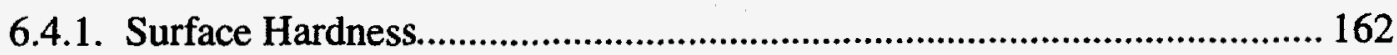

6.4.2. Apparent Fracture Toughness ................................................................. 163

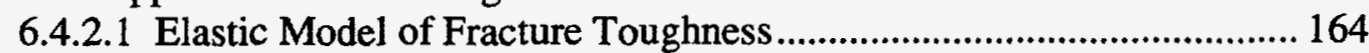

6.4.2.2 Estimation of Surface Energies from Bond Energies............................ 166

6.4.2.3 Estimation of Surface Energies from the Cohesive Strength of a

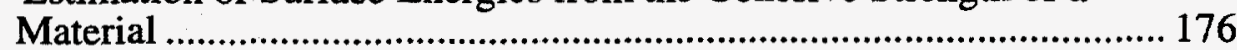

6.4.2.4 Comparison of Surface Energies from Two Models ............................. 179

6.3.2.5 Estimation of $\mathrm{K}_{\mathrm{IC}}$ for Silicon and Diamond.......................................... 180

6.4.2.6 Estimation of Minimum Value of $\mathrm{K}_{\mathrm{lC}}$ for DLC Coatings..................... 183 
6.4.2.7 Estimation of Surface Energy of DLC Coatings .................................... 186

6.4.3. Friction and Wear Behavior.................................................................. 190

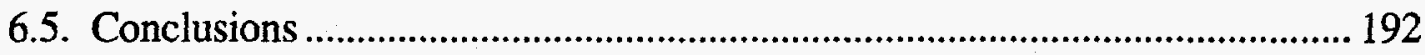

Chapter 7 Investigations of BCN Coatings .......................................................... 194

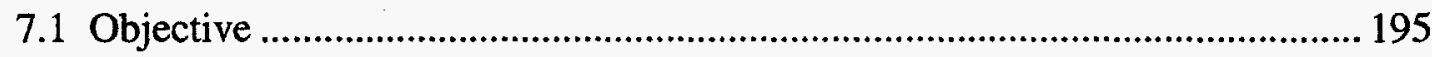

7.2 Synthesis of amorphous BCN Coatings ......................................................... 195

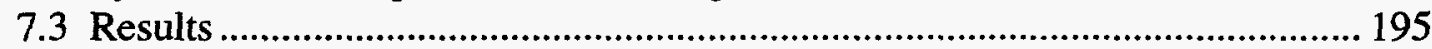

7.3.1 Composition and Microstructure Analysis ................................................ 196

7.3.2 Mechanical Properties......................................................................... 203

7.3.2.1 Surface Hardness ................................................................................203

7.3.2.1 Apparent Fracture Toughness..........................................................203

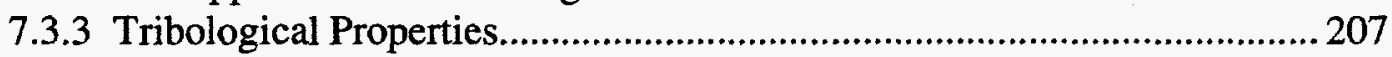

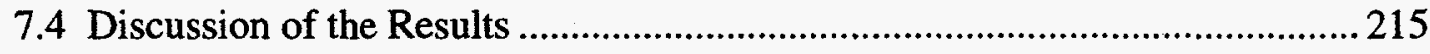

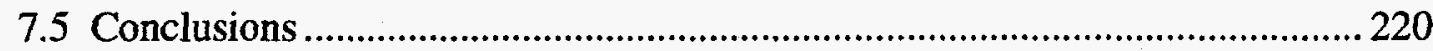

Chapter 8 Summary and Future Proposed Work ................................................ 221

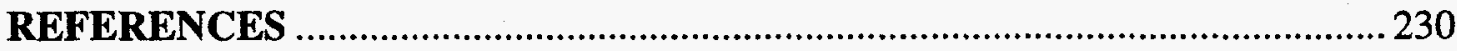




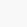




\section{LIST OF TABLES}

Table 2.1 Summary of the parameters used by Sullivan and King [98, 99], Djabella and Amell [100], and Leroy and Villechaise [101] in solving the contact stress fields of layered elastic half-space................................................... 36

Table 2.2 Tribological properties of ion-implanted surfaces.................................... 48

Table $3.1 \rho$ Values as a function of $\varepsilon$ and $k$, from ref. [107] .................................. 56

Table 3.2 Test conditions for tribological investigations. ...................................... 72

Table 3.3 Modulus and Possion's ratio of the sliders............................................... 72

Table 4.1 Results from analytical expressions. ................................................... 81

Table 4.2 Projected range and straggling of carbon and nitrogen ions into $\mathrm{Si}(100)$ from Monte Carlo simulations using TRIM [105]. .................................. 83

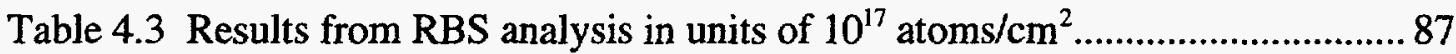

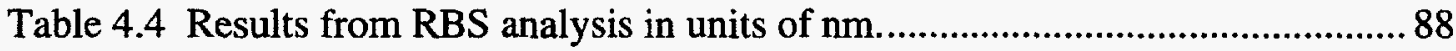

Table 4.5 Width of the ion-modified layer from channeling measurements..............90

Table 4.6 Width of the ion-modified layer from TEM measurements...................... 98

Table 4.7 Roughness values of the implanted and unimplanted $\mathrm{Si}(100)$ samples measured by AFM..................................................................................99

Table 5.1 Hardness, modulus, and wear factor of the unimplanted and implanted $\operatorname{Si}(100)$.

Table 5.2 Roughness values of the implanted and unimplanted $\mathrm{Si}(100)$ samples after wear tests.

Table 5.3 Summary of atomic radii, covalent radii, valency, and electronegativity of $\mathrm{Si}, \mathrm{C}$, and $\mathrm{N}$.

Table 5.4 Summary of activation energy and diffusion constants of $\mathrm{C}$ and $\mathrm{N}$ into $\mathrm{Si}$, $\mathrm{SiC}$, and $\mathrm{Si}_{3} \mathrm{~N}_{4}$......

Table 6.1 Thickness, composition, and intrinsic stresses of various DLC coatings.. 146

Table 6.2 Elastic moduli, hardness, and contact stresses of DLC coatings. 148

Table 6.3 Thickness, hardness, fracture toughness, and wear factor of DLC coatings.151

Table 6.4 Static contact stress, fracture toughness, and wear factor of DLC coatings. 153

Table 6.5 Elastic moduli and hardness values for different coatings. 162 
Table 6.6 Summary of the number of atoms in the plane, area of the plane, and atomic density.

Table 6.7 Summary of the number of bonds broken per atom on one surface and number of bonds per unit area.

Table 6.8 Summary of the number of bonds broken per unit area, estimated surface energy, and measured surface energy.

Table 6.9 Surface energies estimated from the cohesive strength of a material

Table 6.10 Comparison of estimated with measured surface energy values

Table 6.11 Comparison of estimated fracture toughness values and measured fracture toughness.

Table 6.12 Predicted and experimentally determined fracture toughness. 190

Table 7.1 Composition of $\mathrm{BCN}$ coatings. 196

Table 7.2 Summary of hardness and elastic modulus of BCN coatings 204

Table 7.3 Summary of the apparent fracture toughness of $\mathrm{BCN}$ coatings. 206

Table 7.4 Summary of wear factor and the apparent fracture toughness of $\mathrm{BCN}$ coatings.

Table 7.5 Heat of formation data for boron nitride, boron carbide, and born oxide. 218

Table 7.6 Thermodynamic stability of the compound coatings. 219 


\section{LIST OF FIGURES}

Figure 1.1 Wear map of alumina sliding on alumina in dry air from ref. [11]............ 4

Figure 1.2 Comparison of wear transitions for each material under unlubricated

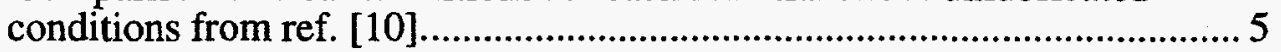

Figure 2.1 Apparent and real area of contact from ref. [1] ................................. 15

Figure 2.2 Relationship between fracture toughness and abrasive wear resistance from ref. [1].

Figure 2.3 Schematic representation of fracture toughness and hardness of plastics, ceramics, and metallic alloys from ref. [1].

Figure 2.4 Illustration of (a) conforming and (b) noncomforming contacts 26

Figure 2.5 Schematic of the stress distribution for the elastic contact of a sphere and a plane. (a) Normal load. (b) Normal load and tangential load.

Figure 2.6 Schematic diagram of the process from loading of the surface to wear..... 30

Figure 2.7 Contour plots of the von Misses stress (normalized to $P_{0}$ ) for the elastic contact of a sphere and a plane: (a) $\mu=0$, (b) $\mu=0.25$, (c) $\mu=0.5$, from ref. $[90,91]$.

Figure 2.8 Influence of friction on the stress distribution of an elastic contact of a sphere and a plane, from ref. $[90,91]$.

Figure 2.9(a) Schematic diagram of an elastic contact of a sphere on a layered medium.

Figure 2.9(b) Illustration of stresses of an elastic contact of a sphere on a layered medium.

Figure 2.10 In-plane stress distribution on the surface of an elastic contact of a sphere with layered elastic half-space: (a) $\mu=0$, (b) $\mu=0.25$, (c) $\mu=0.5$ from ref. $[98,99]$.

Figure 2.11 Shear stress distribution on the surface of an elastic contact of a sphere with layered elastic half-space at $\frac{x}{a_{0}}=0.5$ : (a) $\mu=0$, (b) $\mu=0.25$,

(c) $\mu=0.5$ from ref. $[98,99]$.

Figure 2.12 Stress distribution at $\mathrm{x} / \mathrm{a}=0.5$ for $\mathrm{h} / \mathrm{a}=0.05$ and $\mu=0.5$ from ref. $[98,99] .42$ 
Figure 2.13 Contour plots of the Von Misses stress (normalized to $P_{0}$ ) for $\mu=0.25$ for different coating to substrate modulus ratio and under the same loading conditions. (a) $\mathrm{E}_{\mathrm{c}} / \mathrm{E}_{\mathrm{s}}=0.5$,(b) $\mathrm{E}_{\mathrm{c}} / \mathrm{E}_{\mathrm{s}}=1.0$, (c) $\mathrm{E}_{\mathrm{c}} / \mathrm{E}_{\mathrm{s}}=2.0$ from ref. [98, 99].

Figure 3.1a Illustration of range, $R$, and projected range, $R_{p}$, of an incident ion........51

Figure 3.1b Illustration of Gaussian profile of an implantation process.

Figure 3.1c Relationship between the range, $R$, the projected range, $R_{p}$, and the range straggling, $\Delta R_{p}$, as functions of mass ratio, $M_{2} / M_{1} . \Delta R_{p x}$ is in the direction of the incident ion, and $\Delta R_{p y}$ is in the perpendicular direction of the incident ion. Dashed lines, $m=1 / 3$, solid lines, $m=1 / 2$, from ref. [16]...... 57

Figure 3.1d Schematic diagram of beam-line ion implanter. 58

Figure 3.2 Schematic diagram of plasma-source ion implantation, from ref. [16] .... 60

Figure 3.3 Schematic diagram of DC magnetron sputtering. 62

Figure 3.4 Flow chart to evaluate the tribological performance of a coating. .64

Figure 3.5 Schematic diagram of a backscattering spectrometry accelerator. 66

Figure 3.6a Schematic diagram of the nanoindenter setup, from ref. [129]. 69

Figure 3.6b Typical loading and unloading curve of an indentation test. 69

Figure 3.7 Schematic diagram of a POD apparatus to measure friction and wear...... 73

Figure 3.8(a) Deformation and fracture due to Vickers indentation (side view).

(b) Indentation pattern (plane view).

Figure 4.1 Implanted profile of carbon and nitrogen ions from Monte Carlo simulations.

Figure 4.2a Rutherford backscattering spectra of the sample implanted with $4 \times 10^{17} \mathrm{C}^{+}$ions $/ \mathrm{cm}^{2}$.

Figure 4.2b Rutherford backscattering spectra of the sample implanted with $8 \times 10^{17} \mathrm{C}^{+}$ions $/ \mathrm{cm}^{2}$.

Figure 4.3a Channeling spectra of the sample implanted with $4 \times 10^{17} \mathrm{~N}^{+}$ions $/ \mathrm{cm}^{2}$ and the uncoated silicon.

Figure 4.3b Channeling spectra of the sample implanted with $8 \times 10^{17} \mathrm{~N}^{+}$ions $/ \mathrm{cm}^{2}$ and the uncoated silicon. 92

Figure 4.4 Selected area diffraction pattern of the modified layers for $4 \times 10^{17} \mathrm{C}^{+}$ions $/ \mathrm{cm}^{2}$. .93

Figure 4.5 TEM bright-field image of $4 \times 10^{17} \mathrm{~N}^{+}$ions $/ \mathrm{cm}^{2}$. 94 


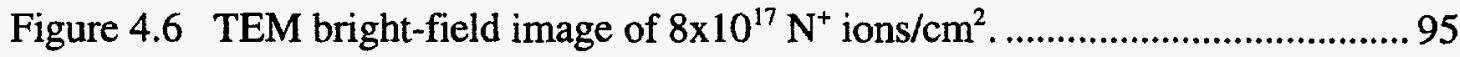

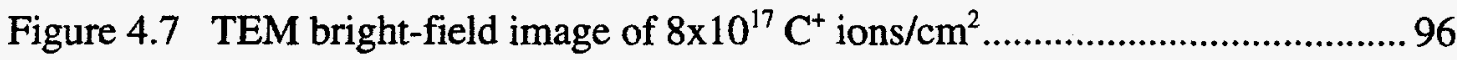

Figure 4.8 TEM bright-field image of $4 \times 10^{17} \mathrm{C}^{+}$ions $/ \mathrm{cm}^{2}+4 \times 10^{17} \mathrm{~N}^{+}$ions $/ \mathrm{cm}^{2} \ldots . .97$

Figure 4.9 Atomic force microscopy images (a) Unimplanted

(b) $4 \times 10^{17} \mathrm{C}^{+}$ions $/ \mathrm{cm}^{2}$

Figure 4.9 Atomic force microscopy images (c) $4 \times 10^{17} \mathrm{~N}^{+}$ions $/ \mathrm{cm}^{2}$

(d) $4 \times 10^{17} \mathrm{C}^{+}$ions $/ \mathrm{cm}^{2}+4 \times 10^{17} \mathrm{~N}^{+}$ions $/ \mathrm{cm}^{2}$

Figure 4.9 Atomic force microscopy images (e) $8 \times 10^{17} \mathrm{C}^{+}$ions $/ \mathrm{cm}^{2}$

(f) $8 \times 10^{17} \mathrm{~N}^{+}$ions $/ \mathrm{cm}^{2}$

Figure 5.1 Surface hardness as a function of depth for nitrogen-implanted samples. 110

Figure 5.2 Surface hardness as a function of depth for carbon-implanted samples. 112

Figure 5.3 Coefficient of friction as a function of cycles for nitrogen-implanted samples.

Figure 5.4 Coefficient of friction as a function of cycles for carbon-implanted samples.

Figure 5.5 SEMs showing the morphology of the wear tracks after 150 cycles

(a) $4 \times 10^{17} \mathrm{C}^{+}$ions $/ \mathrm{cm}^{2}$ (b) $8 \times 10^{17} \mathrm{~N}^{+}$ions $/ \mathrm{cm}^{2}$.

Figure 5.6 SEMs of the wear tracks after 660 cycles (a) Unimplanted

(b) $4 \times 10^{17} \mathrm{C}^{+}$ions $/ \mathrm{cm}^{2}$ (c) $8 \times 10^{17} \mathrm{C}^{+}$ions $/ \mathrm{cm}^{2}$

Figure 5.7 SEMs of the wear tracks after 660 cycles (a) Unimplanted

(b) $4 \times 10^{17} \mathrm{~N}^{+}$ions $/ \mathrm{cm}^{2}$ (c) $8 \times 10^{17} \mathrm{~N}^{+}$ions $/ \mathrm{cm}^{2}$.

Figure 5.8 Wear track profiles for $\mathrm{Si}(100)$ after 150 cycles (a) Unimplanted

(b) $4 \times 10^{17} \mathrm{C}^{+}$ions $/ \mathrm{cm}^{2}$ (c) $4 \times 10^{17} \mathrm{C}^{+}$ions $/ \mathrm{cm}^{2}+4 \times 10^{17} \mathrm{~N}^{+}$ions $/ \mathrm{cm}^{2}$

(d) $4 \times 10^{17} \mathrm{~N}^{+}$ions $/ \mathrm{cm}^{2}$

Figure 5.9 Wear track profiles for $\mathrm{Si}(100)$ after 660 cycles (a) Unimplanted

(b) $4 \times 10^{17} \mathrm{C}^{+}$ions $/ \mathrm{cm}^{2}$ (c) $8 \times 10^{17} \mathrm{C}^{+}$ions $/ \mathrm{cm}^{2}$ (d) $4 \times 10^{17} \mathrm{~N}^{+}$ions $/ \mathrm{cm}^{2}$

(e) $4 \times 10^{17} \mathrm{C}^{+}$ions $/ \mathrm{cm}^{2}+4 \times 10^{17} \mathrm{~N}^{+}$ions $/ \mathrm{cm}^{2}$

Figure 5.10 Wear factor as a function of different species and doses for 150 cycles.124

Figure 5.11 Wear factor as a function of different species and doses for 660 cycles.125

Figure 5.12 Wear factor for 660 cycles as a function of hardness.

Figure 5.13 Structure of silicon.

Figure 6.1 ERD spectra of sample S2.

Figure 6.2 Dependence of hardness on coating thickness. 
Figure 6.3 Scanning electron micrographs of the indents at 1000 grams

(a) Uncoated silicon (b) Sample 4

Figure 6.4 Results from the data of well-defined radial crack in uncoated silicon and DLC coating as a function of an applied load. 152

Figure 6.5 Dependence of apparent fracture toughness on coating thickness.. 154

Figure 6.6 Dependence of apparent fracture toughness on hardness of the coatings.155

Figure 6.7 Scanning electron micrograph of the indent on the sample S6 at 2000 grams.

Figure 6.8 Coefficient of friction of sample 4 (S4) and uncoated silicon at $6.25 \mathrm{~N}$ with ruby pin.

Figure 6.9 Scanning electron micrographs of the wear tracks (a) Uncoated silicon

(b) Sample 4 .

Figure 6.10 Dependence of apparent fracture toughness on wear factor.

Figure 6.11 Wear track profiles of uncoated and coated samples

(a) Uncoated silicon (b) Sample S3 (c) Sample S6.

Figure 6.12(a) Planar atomic configuration of Si(100) viewed along [100].

(b) Cleavage along the face of the $\mathrm{Si}(100)$ between plane 1 and plane 2.168

Figure 6.13(a) Planar atomic configuration of $\mathrm{Si}(110)$ viewed along [110].

(b) Projected perspective view of the above configuration along $[-1,-1,0]$.

(c) Projection of the figure shown in (b) along [001]

Figure 6.14(a) Planar atomic configuration of $\mathrm{Si}(111)$ viewed along [111].

(b) Side view of above configuration projected along $[1,1,-2]$..

Figure 6.15(a) Potential energy between atoms in a crystal as a function of interatomic distance. (b) Force between atoms as a function of inter-atomic distance.

Figure 6.16 Half -penny crack of a coating-substrate system viewed in cross-section.

Figure 7.1a Rutherford backscattering spectra of the sample BCN coating along with simulated spectra for Sample 4 .

Figure 7.1b Rutherford backscattering spectra of the sample BCN coating Sample 4 (expanded to show the lighter elements).

Figure 7.2 Rutherford backscattering spectra of the BCN coatings (Samples 4 and 5).

Figure 7.3 Composition triangle of the $\mathrm{BCN}$ coatings. 201

Figure 7.4 Selected area diffraction pattern of the BCN coatings. 202 
Figure 7.5 Surface hardness at a depth of $47 \mathrm{~nm}$ as a function of at.\% of nitrogen.. 205

Figure 7.6 Results from the data of well-defined radial cracks as a function of an applied load.(a) Uncoated silicon and Sample 1. ........................................ 208

Figure 7.7 Coefficient of friction. (a) Uncoated silicon at $0.8 \mathrm{~N}$ with ruby pin....... 209

Figure 7.7 Coefficient of friction. (b) Sample 1 at $0.8 \mathrm{~N}$ with ruby pin....................2. 210

Figure 7.7 Coefficient of friction. (c) Sample 5 at $0.8 \mathrm{~N}$ with ruby pin.................... 211

Figure 7.8 Scanning electron micrograph of the wear track of the uncoated silicon 212

Figure 7.9 Scanning electron micrographs showing the wear morphology of

(a) Sample 1 (b) Sample 5.

Figure 7.10 Wear track profiles after 10,000 cycles. (a) Uncoated silicon

(b) Sample 1 (c) Sample 2 (d) Sample 3 (e) Sample 4 (f) Sample 5.. 214

Figure 7.11 Wear factor as a function of hardness. 
xviii 


\title{
MECHANICAL AND TRIBOLOGICAL PROPERTIES \\ OF ION BEAM-PROCESSED SURFACES
}

\author{
by \\ Padma Kodali
}

\begin{abstract}
A variety of surface modification and surface coating techniques are currently used in industry to modify the near-surface mechanical properties that influence the friction and wear behavior of metals, metallic alloys, ceramics, and polymers. Nearsurface mechanical properties such as hardness and fracture toughness of a coatingsubstrate system can be tailored economically without changing the bulk properties of the system.

The intent of this work was to broaden the applications of well-established surface modification techniques and to elucidate the various wear mechanisms that occur in sliding contact of ion-beam processed surfaces. The investigation included characterization and evaluation of coatings and modified surfaces synthesized by three surface engineering methods; namely, beam-line ion implantation, plasma-source ion implantation, and DC magnetron sputtering. Correlation among measured properties such as surface hardness, fracture toughness, and wear behavior was also examined.

This dissertation focused on the following areas of research:

- Investigating the mechanical and tribological properties of mixed implantation of carbon and nitrogen into single crystal silicon by beam-line implantation.

- Characterizing the mechanical and tribological properties of diamond-like carbon (DLC) coatings processed by plasma source ion implantation.

- Developing and evaluating metastable boron-carbon-nitrogen (BCN) compound coatings for mechanical and tribological properties.

The surface hardness of a mixed carbon-nitrogen implant sample improved significantly compared to the unimplanted sample. However, the enhancement in the wear factor of this sample was found to be less significant than carbonimplanted samples. The presence of nitrogen might be responsible for the degraded wear behavior since nitrogen-implantation alone resulted in no improvement in the wear factor. Wear mechanisms that occurred in implanted and unimplanted surfaces tested against AISI52100 steel were determined to be adhesive, abrasive, and third body wear.
\end{abstract}


DLC coatings have low friction, low wear factor, and high hardness. The fracture toughness of DLC coatings has been estimated for the first time. The wear mechanism in DLC coatings investigated with a ruby slider under a contact stress of $1 \mathrm{GPa}$ was determined to be plastic deformation.

The preliminary data on metastable $\mathrm{BCN}$ compound coatings indicated high friction, low wear factor, and high hardness. The wear morphology of the coatings that were tested against a ruby slider suggested that abrasive wear, brittle fracture, and third body wear are the mechanisms by which loss of material can occur. 


\section{Chapter 1 Introduction}

Tribology is an interdisciplinary subject involving the science and technology of interacting interfaces in relative motion and embracing the study of friction, wear, and lubrication. Mechanical properties such as hardness, elastic modulus, and fracture toughness influence the friction and wear behavior of interacting surfaces. The ambient environment and loading conditions also influence the friction and wear properties of sliding or moving surfaces. Thus, friction and wear are characteristic properties of an engineering system.

Friction and wear of bulk materials can be modified by applying coatings that can change the near-surface properties. Potential applications of new coatings depend on their tribological performance at the desired operating conditions and the nature of the interacting surfaces. Accordingly, understanding the relationship between tribological and mechanical properties is crucial in developing effective coatings.

\subsection{Friction and Wear}

Issues of friction and wear have been reviewed in a number of text books [1-8]. Friction and wear are complex processes that involve the mechanical and/or chemical interaction of material surfaces in sliding or rolling contact. At a macroscopic level, friction and wear occur simultaneously, whereas at the atomic level, friction can occur without wear [9].

The chemical interaction of contacting surfaces may lead to adhesion. Thermodynamically derived adhesive forces that operate when the contacting surfaces come into contact form bonds between the two surfaces. Both friction and wear involve the separation of these adhesive bonds. The energy losses in breaking these adhesive bonds are concentrated at relatively thin interface zones. 
The energy dissipated in mechanical interactions involve much larger volumes of material compared to adhesion and may extend to the subsurface. The depth and severity of subsurface damage depends on the applied load and type of material. Mechanical interaction of surfaces can be either elastic, plastic, or both. Elastic deformation of the contacting asperities involves no permanent surface deformation, whereas plastic deformation involves surface change. The extent and type of damage depend on the loading conditions and material type.

Attempts have been made to relate the wear damage-related phenomena such as plastic deformation and fracture to macroscopic operating parameters such as pressure and velocity. Correlation between wear and operating parameters led to the construction of maps showing the different regimes of wear [9].

\subsection{Wear Maps}

Wear maps in general indicate the location of different wear regimes with a dominant mechanism of wear. These wear maps imply that wear mechanisms change with tribological test parameters such as velocity, load, and humidity. For example, by increasing the load and velocity of test conditions, the mechanism that is responsible for loss of material can change from plastic deformation to brittle fracture. For a given slider and a set of operating conditions (that is, load and speed), a wear map can be used to help in predicting the underlying mechanisms by which material loss can occur during sliding contact.

Lim and Ashby [9] constructed wear maps for steels against steels under dry sliding conditions. They constructed these maps empirically and by modelling. They identified various wear mechanisms: plasticity-dominated wear, which includes mild, severe, and delamination wear; oxidation-dominated wear; melt-dominated wear; and seizure. They mapped different regimes where each of these wear mechanisms is dominant. In different regimes, the interfacial temperature caused by frictional 
heating is responsible for the various wear mechanisms. The calculated data was in good agreement with experiental observations.

Hsu et al. [10-15] extensively and systematically studied the wear mechanisms that are common to ceramics. They used the data to construct wear maps for ceramics under lubricated and unlubricated conditions. Figure 1.1 is an example of a simplified approach developed by Hsu et al. [11] for relating the applied load and sliding speed to the wear mechanisms that occur in alumina without lubrication. The transition from ultra-mild wear to mild wear occurs either when the operating load exceeds $1 \mathrm{~N}$ or when the sliding speed exceeds $0.1 \mathrm{~m} / \mathrm{sec}$.

Design engineers can use information derived from the wear maps in the initial stages of selecting a coating material. Figure 1.2 shows the transient points where the mild plastic wear mechanism changes to a fracture-controlled mechanism for four different ceramic materials for dry sliding conditions and at a combined load and speed condition. The approximate transition regimes are bounded by the parallel lines. For example, at a load of $5 \mathrm{~N}$ and sliding speed of $0.01 \mathrm{~m} / \mathrm{sec}$, alumina is a better choice than zirconia for minimizing wear under dry sliding conditions.

Thus, identifying the dominant wear mechanism for the desired operating loading conditions from wear maps may help in choosing a suitable material. If similar wear maps were developed for coatings, selection of appropriate materials for desired applications would be simplified.

Friction and wear are not intrinsic material properties, but they are characteristics of an engineering system. As mentioned earlier, these properties are strongly influenced by the ambient environment. Friction and wear of a substrate can be changed by modifying the near-surfaces properties. Surface engineering is the branch of science that deals with tailoring the properties of surfaces to improve tribological or corrosion properties. 


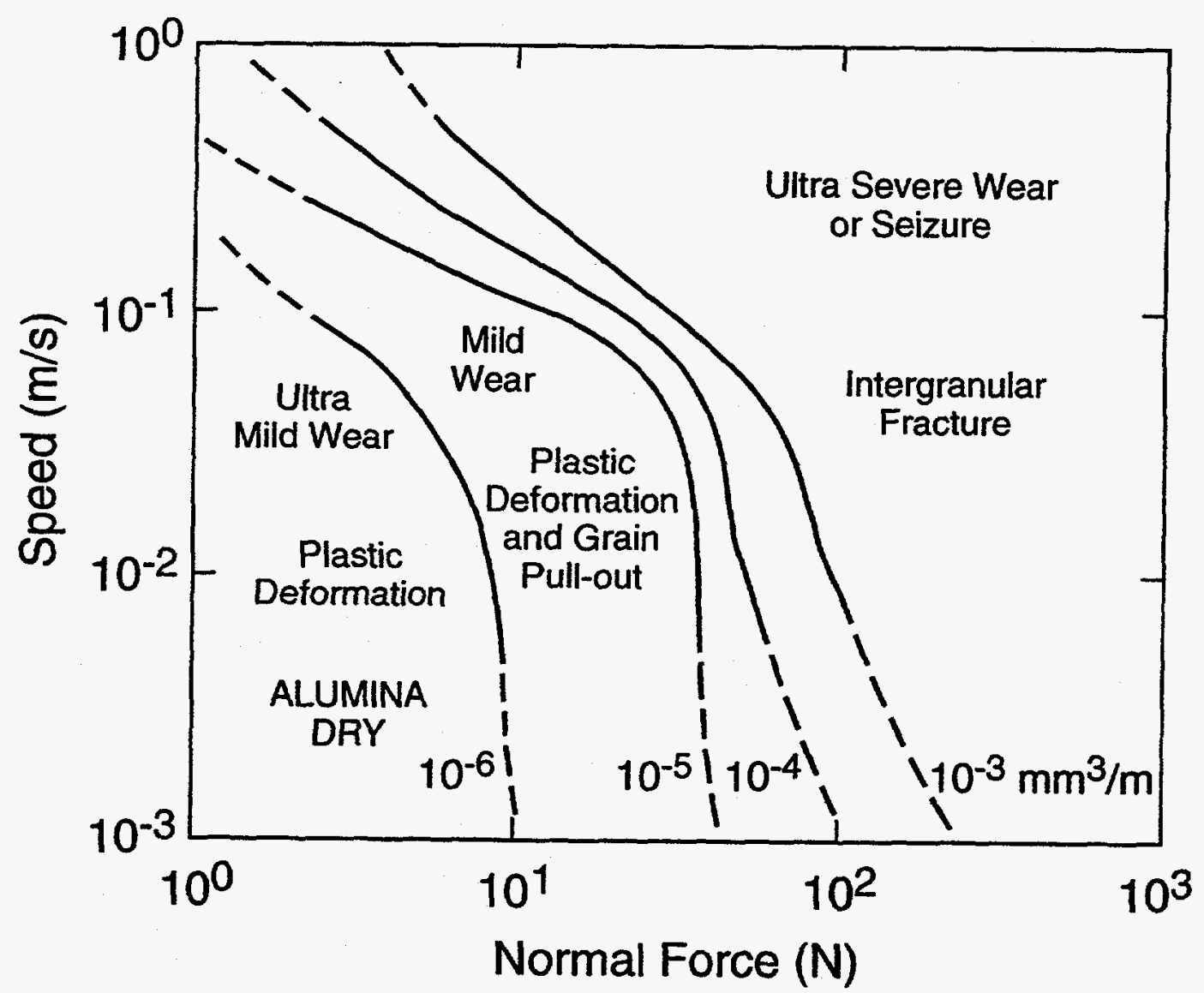

Figure 1.1. Wear map of alumina sliding on alumina in dry air from ref. [11]. 


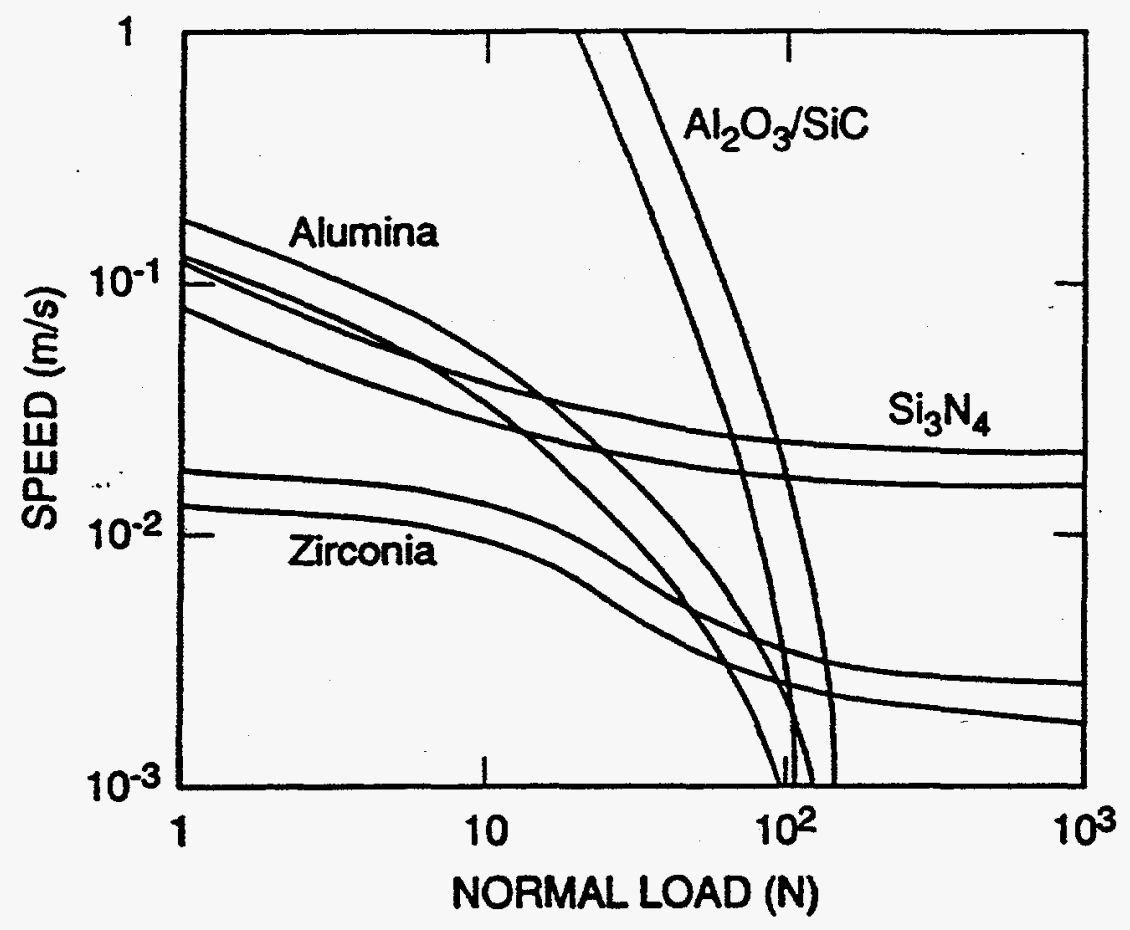

Figure 1.2. Comparison among wear transitions for each material unlubricated conditions from ref. [10]. 


\subsection{Surface Engineering}

Two common approaches to control wear and friction are surface modification and surface coating. In surface modification, the original surface is changed, whereas in surface coating, the new material is added onto the surface. The purpose of surface engineering is to modify the surface to achieve a set of specific properties for an application. For example, the substrate material can be designed for strength and toughness, while the coating is responsible for both wear and corrosion resistance. Improved wear resistance of the modified surfaces is attributed to changes in the composition and microstructure of the near-surface region. These changes, in turn, affect mechanical properties such as hardness, toughness, and flow strength of the surface layer. For example, if the modified layer is hard, wear by abrasion is minimized. By reducing abrasion at the surface, the total frictional force is decreased. If there are any intrinsic residual compressive stresses caused by processing, they retard crack propagation and can reduce the formation of wear debris. Thus, a less expensive substrate like aluminum can be modified at the surface to perform as well as an expensive ceramic such as aluminum nitride. Details of surface engineering are discussed in the next chapter.

\subsection{Objective of this Dissertation}

This research was intended to contribute to an understanding of surface engineering methods and the various wear mechanisms that can occur in the sliding contact of ion beam-processed surfaces. The investigation included characterization and evaluation of coatings synthesized by three surface engineering methods; namely, beam-line ion implantation, plasma-source ion implantation, and DC magnetron sputtering.

Beam-line ion implantation has proven to be a viable technique to tailor friction and wear properties of metals, ceramics, and polymers [16-22]. Processing 
parameters such as ion species, dose, energy, and the temperature at which the implantation was perfomed influence the properties of the modified region.

In the present semiconductor technology, silicon is the dominant material used in microelectronic and micromechanical devices because of its excellent processing properties. There are several reports aimed at understanding the friction and wear behavior of surface-modified silicon by implantation of a single ion species, both at a microscopic and macroscopic level [23-26]. This research was undertaken to study the role of implantation of multiple ion species into silicon. The tribological properties of silicon implanted with single implants of carbon and nitrogen as well as a combination of the two were studied. Carbon and nitrogen ions were chosen because carbides and nitrides have high hardness and good wear resistance.

Plasma-source ion implantation (PSII) is a relatively new processing technique that was initially developed to overcome the limitations of traditional beam-line implantation, such as target manipulation, and to produce high doses of ions in a simple, fast, efficient, and cost-effective manner [27-31]. PSII has been successfully used to improve the tribological properties of a variety of steels and metals [32-42].

Diamond-like carbon coatings (DLC) have attracted great attention in the last two decades. Because of the low friction and wear of DLC coatings, potential applications to increase the performance and lifetime of cutting tools, gears, bearings, seals, cams, etc. are numerous. In addition to the low friction and wear of these coatings, their excellent biocompatibility has had an impact in biomedical applications [43]. Several researchers have performed extensive studies on the tribological properties of amorphous DLC coatings processed by various techniques [33, 44-53]. However, there are no reports on the apparent fracture toughness of these amorphous coatings nor on its role on the tribological properties of these coatings. The present study also investigated amorphous DLC coatings synthesized by PSII on a silicon substrate. The apparent fracture toughness, hardness, and tribological properties of 
these coatings were studied. The wear behavior of the DLC coatings was examined as a function of the hardness, apparent fracture toughness, stress, and thickness.

DC magnetron sputtering is widely used in industries to synthesize ceramic or metallic coatings. In this investigation, an approach to develop novel coatings with high fracture toughness and high hardness using this processing technique was attempted. Hard wear-resistant coatings often fail by brittle fracture because of their low fracture toughness. The addition of a softer phase may improve their fracture toughness. Coatings with a mixture of hard and soft phases are known as compound coatings. Compound coatings can have both a high fracture toughness and a high hardness.

In search of new coatings with high fracture toughness and high hardness, this research explored metastable boron-carbon-nitrogen $(\mathrm{BCN})$ compound coatings to see if the combination of the lubricating nature of crystalline hexagonal boron nitride and the wear resistance of boron carbide could be combined. The hardnesses of polycrystalline boron carbide and hexagonal boron nitride are reported to be $40 \mathrm{GPa}$ and $9 \mathrm{GPa}$, respectively [55]. In addition to high hardness, polycrystalline boron carbide is also reported to have a high wear resistance [55]. Hexagonal boron nitride has a structure similar to graphite and can act as a solid lubricant. In this work, amorphous $\mathrm{BCN}$ coatings synthesized by $\mathrm{DC}$ magnetron sputtering have been characterized for mechanical and tribological properties for the first time.

\subsection{Methodology}

The methodology of this research was to characterize surface coatings for composition, microstructure, hardness, fracture toughness, and tribological properties. For hardness measurements, a nanoindenter was used; for fracture toughness measurement, the Vickers microindenter was used. Tribological tests were conducted with pin-on-disc (POD) tests using both AISI 52100 steel pins and ruby pins under dry sliding conditions and controlled humidity. Composition of the ion-modified surfaces 
was determined using ion-beam analysis, and microstructure was characterized with transmission electron microscopy.

Basic wear mechanisms were determined for each type of coating, and wear rates were compared with those of the unmodified substrate. The results are discussed in relation to current models in the literature. The experimental techniques that were used to characterize the coatings are discussed in detail in the Chapter 3.

\subsection{Summary}

This dissertation focused first on understanding surface engineering methods, then on using these methods to modify friction and wear properties of a silicon substrate. The importance of characterizing these modified surfaces for composition, microstructure, and mechanical properties by appropriate techniques and correlating the measured properties to evaluate a coating performance was demonstrated. 


\section{Chapter 2 Overview of Tribology and Surface Engineering}

Tribology is a branch of science dealing with the interactions of contacting bodies that are in relative motion. These interactions control friction and wear properties of the materials that are in contact. Friction and wear are not intrinsic material properties but are characteristics of an engineering system. Tribological contact induces physical and chemical changes on the contact surfaces, and these changes occur at random. These random occurrences during a contact make the tribological investigations complex.

This chapter describes the importance of tribology, theories of friction and wear, contact mechanics, and surface engineering. As will be described in the experimental portion of this thesis, tribological investigations were performed using a pin-on-disc (POD) apparatus. In this setup, a spherical slider rests on a flat rotating disc. Therefore, the stresses and deformations on an elastic half-space loaded by a sphere will be discussed.

\subsection{Importance of Tribology}

Reliability and longevity of mechanical, electrical, and electronic equipment are continuously challenged for several reasons, including minimum energy use, maximum productivity, and ensured safety of operation. Friction and wear contribute to energy losses in industrial equipment. In general, friction and wear processes occur jointly when two surfaces undergo sliding or rolling contact under load. Friction is the resistance encountered by one body in moving over another. Wear is defined as the progressive loss of material from the operating surface of a body as a result of the relative motion between surfaces. 
Design engineers are challenged to tailor friction and wear to the needs of an application of an engineering component. In certain applications, such as in bearings and gears, low friction is essential; while in others, such as clutches and belt drives, high friction and low wear are desired. In the magnetic recording industry, tribology is the central technical problem. For example, inside a video cassette recorder (VCR), the tape and head are in relative motion. There is an air gap of about $30 \mathrm{~nm}$ between the head and tape. The penetration depth (stored information density) can be increased by decreasing this air gap. While decreasing the air gap yields higher signal strength, it also results in greater tape wear. In high-density tapes, occasionally the head and the tape come in contact, resulting in tape wear. This wear can cause the tape to lose information. With an increase in the number of contacts, wear debris accumulates on the head, resulting in damage to the tape and head. Thus, high-interface reliability between the tape and head through the whole life of operation is critical. Greater advances in information storage density would result from the solution of tribological issues of the tape and the head.

Tribology is also an important problem in the health and medical field. The wear of brackets, pins, and plates in dental bridges and orthopedic implants such as knee and hip replacements illustrate the profound impact of tribological issues on our lives. Materials such as titanium, titanium alloys, cobalt chromium alloys, and stainless steels are widely used as implant materials. Hip implants, for example, can only function for about 15 years. If the hip implant replacement surgery is performed on a younger patient, the patient may need a second implant later in life if the implant wears out too soon. The critical factor limiting the life of an implant is the wear debris accumulated with use.

A common problem in each of these examples is the control of friction and wear. In absence of surface lubricating layers, tribological phenomena are related to the fundamental properties of materials, such as bonding, hardness, modulus, tensile 
strength, toughness, and fatigue limit. The tribology problem needs to be addressed by a multidisciplinary approach. To solve a tribological problem, knowledge in the following area is required.

- A sound knowledge of contact mechanics dealing with stress distribution of loaded contacts. (This part of the problem predicts the areas of the contact, pressure, temperature distribution, flow stress distributions, crack formation, and particle detachment of the loaded contacts.)

- Mechanical properties of materials in contact.

The knowledge of material properties such as Young's modulus, Poisson's ratio, coefficient of thermal expansion, and thermal conductivity is useful to interpret the tribological behavior of a system. For example, the contact radius of an asperity and it's contact pressure that will be discussed later in this chapter are a function of Young's modulus and Poisson's ratio.

Traditionally, the performance of engineering components could be improved by proper material selection and design improvement. Surface-engineered materials are among some of the new materials that have been developed in the last few decades. In this class of materials, only the near-surface properties of the material are modified. The choice of modification technique is based on four important principles:

- the bulk material to be modified should be chemically stable during the processing steps,

- the bulk material should retain its properties even after surface modification,

- the process should be cost effective, and

- environmental hazards should be kept to an acceptable level. 
Depending on the application, a thin and either a soft or hard coating is used in sliding and rolling bearings [55]. Hard coatings like titanium nitride (TiN) improve the lifetime of cutting tools [56]. In the information technology sector, various soft coatings such as molybdenum sulfide $\left(\mathrm{MoS}_{2}\right)$, carbon (C), and silver ( $\left.\mathrm{Ag}\right)$ on the head and the magnetic tape have proved to be efficient in reducing friction and wear [55].

In biomedical components, various coatings such as hydroxyapatite (calcium phosphate) are used to facilitate direct bonding to bone [55]. Titanium and titanium alloys modified by nitrogen ion implantation have also been investigated for improved life and biocompatibilty [55]. Another promising coating is amorphous diamond-like carbon (DLC) coatings that have a low coefficient of friction and are both wear resistant and inert to human tissue [43]. Moreover, DLC coatings adhere strongly to existing implant materials [57].

\subsection{Tribological Contact of Surfaces}

This section gives a brief description of the interactions of surfaces in contact. When two solid surfaces are brought together, contact generally occurs at a few asperities (contact points) as shown in Figure 2.1. The sum of these local contact areas represents the real area of the contact, which is only a fraction of the apparent area of the contact. The number of asperities that come in contact increases with the normal load, $\mathrm{P}$. These asperities are responsible for supporting the normal load on the surface and for generating frictional forces. The contact pressure exerted by a normally loaded surface on the contact depends on the contact geometry. Greenwood and Williamson [58] proposed a measure of the deformation of surface asperities by using a dimensionless quantity; the plasticity index, $\psi$. Greenwood and Williamson [58] assumed that the asperities had a Gaussian height distribution and were all of the same tip radius. They also assumed that the elastic deformation and stresses could be calculated from the Hertzian equations. The plastcity index, $\psi$, is given by 


$$
\psi=\frac{E_{c}}{H}\left(\frac{\sigma_{p}}{\beta}\right)^{0.5}
$$

with composite modulus $\mathrm{E}_{\mathrm{c}}$,

$$
E_{c}=\left[\frac{1-v_{1}^{2}}{E_{1}}+\frac{1-v_{2}^{2}}{E_{2}}\right]^{-1}
$$

where:

$$
\sigma_{p}=\left(\sigma_{1}^{2}+\sigma_{2}^{2}\right)^{1 / 2} \text { is the composite standard deviation of the asperity peak }
$$

heights, where $\sigma_{1}$ and $\sigma_{2}$ are the root mean square (RMS) roughness of the surfaces 1 and 2, respectively.

$\beta=1 /\left(1 / \beta_{1}+1 / \beta_{2}\right)$ is the composite mean radius of curvature, where $\beta_{1}$ and $\beta_{2}$ are the radius of curvature of contacting asperities of the surfaces 1 and 2 , respectively.

$\mathrm{E}_{1}$ and $v_{1}, \mathrm{E}_{2}$ and $v_{2}$ are the Young's modulus and Poisson's ratio of surfaces 1 and 2 , respectively.

$\mathrm{H}$ is the hardness of the softer material.

The plasticity index combines mechanical properties $(\mathrm{E}$ and $\mathrm{H})$ and topographical properties ( $\sigma$ and $\beta$ ) of the surfaces in contact. Greenwood and Williamson [58] found that the value of $\Psi$ may vary from 0.1 to 100 for real surfaces. In practice, it falls in a narrow range. The deformation is predominantly elastic if the value of $\Psi$ is $<0.6$ and plastic if $\Psi \geq 1.0$. The deformation is elasto-plastic for $0.6<\Psi<1.0$.

According to this model, load does not enter the expression for the plasticity index. Surface properties and surface topography play an important role in determining the occurrence of the plastic deformation. If the deformation is initially plastic, surface interaction in repeated passes may smooth the surface during run-in 


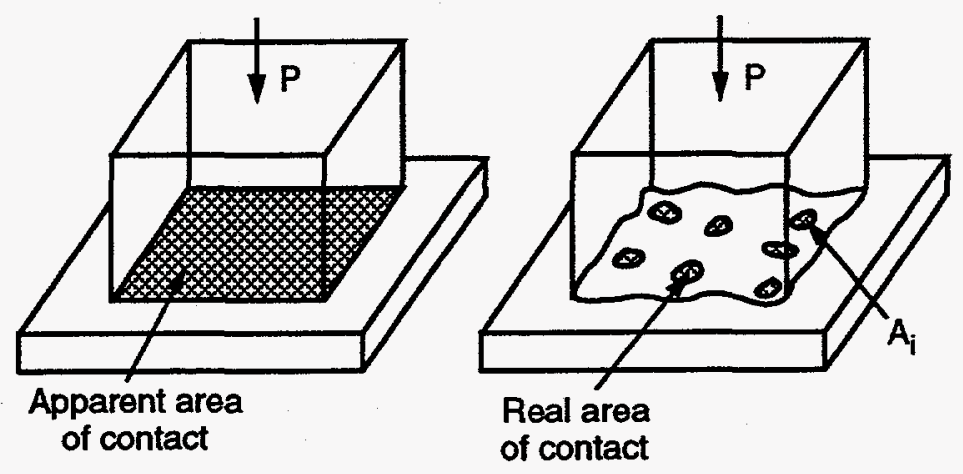

Static Contact

Sliding Contact
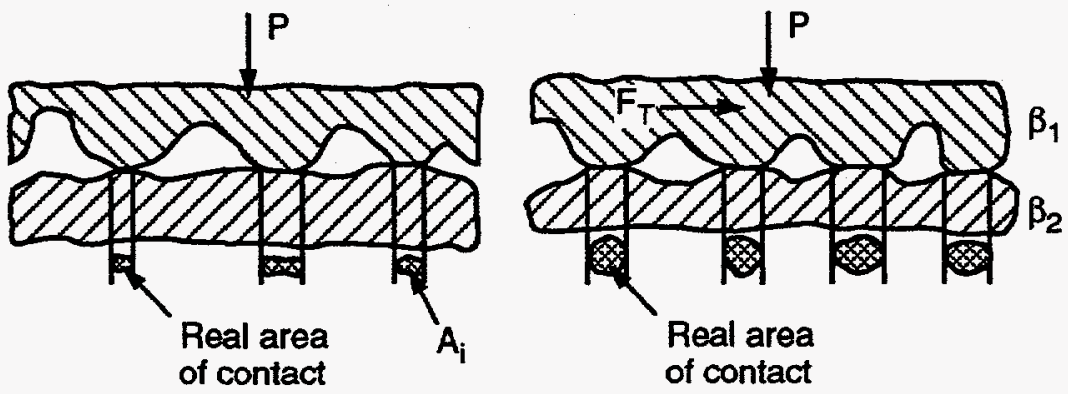

Real area

of contact

Figure 2.1. Apparent and real area of contact from ref. [1]. 
until the standard deviation of the asperity peak height distribution decreases and/or the radius of curvature of the apserities increases, such that the plasticity index falls into the elastic range of contact.

\subsection{Theories of Friction and Wear}

In general, friction and wear processes occur simultaneously when two surfaces undergo sliding or rolling contact under load. Friction is the resistance encountered by one body in moving over the another. Wear, which will be discussed in the later part of this chapter, is defined as the progressive loss of material from the operating surface of a body as a result of the relative motion between surfaces.

\subsubsection{Friction}

In general, no engineering surface is perfectly smooth, regardless of the surface finish method employed. Roughness is a measure of the asperity distribution. Theories of friction are based on adhesion, deformation, or a combination of both. Friction is not a constant for any given material, but it is a function of both materials that are in contact. These two materials may be chemically similar or dissimilar. The environment also has strong influence on friction. Adhesion and deformation are due to the natural chemical and mechanical interaction of the asperities, respectively.

In the adhesion model of friction by Bowden and Tabor $[59,60]$, atomic interactions between asperities subjected to contact loading lead to the formation of strong bonds at the contacts. The area over which the two sliding asperities are bonded is called the adhesive junction. Adhesion of surfaces at ambient conditions is still controversial. Contaminant layers on the surface make the formation of these junctions in air and at room temperature almost impossible with the exception of noble metals. However, even at relatively low loads, contact pressures can be sufficiently high to penetrate these surface layers and initiate plastic deformation locally resulting in adhesive junction formation. When a tangential force is introduced, individual asperities deform under the combined actions of both normal and shear stresses. The 
frictional force caused by adhesion is simply a product of shear strength and contact area. First-order calculations with this relation gives friction coefficients of the order of 0.2 ; however, experimentally determined values are much greater than this value. Several researchers developed a number of theories to explain the discrepancy between theoretically predicted and experimentally observed values of friction [6165]. Even though these models accounted for additional components of friction from ploughing, roughness, and surface energies of adhesion, none of them were successful in explaining experimentally observed values of the friction.

In addition to adhesion, deformation associated with a sliding/rolling contact can contribute to frictional forces. In deformation theory, mechanical interactions caused by the asperities may involve deformation processes that can be elastic, plastic, or both. Several models relate friction with various deformation processes [65-70]. As mentioned earlier, the common features of all these models are adhesion and deformation processes by which the energy is dissipated by generating frictional force. The models deal with several routes possible for each process. One deformation model of friction is summarized as an example. Rigney and Hirth [69] related frictional force for steady state friction under simple sliding situations to the amount of plastic deformation by the following equation.

$$
\mu=\frac{w \tau \varepsilon}{L}
$$

where:

$\mathrm{L}$ is load,

$\tau \varepsilon$ is plastic work done per unit volume,

$t$ is thickness of the deformed zone, $\mu$ is the coefficient friction, 
w is width of deformed zone,

$\tau$ is shear stress, and

$\varepsilon$ is strain per unit cycle.

In Eqn [2.3], w, t, and $L$ can be obtained from the measurements for a given sliding contact. However, shear stress $\tau$ and $\varepsilon$ average strain per cycle are not readily

available. These quantities ( $\tau$ and $\varepsilon$ ) can be estimated from the published data in the literature. Thus, prediction of friction coefficients is possible. This model also predicts that at a fixed load, the metal with lower hardness may have higher friction than a metal with higher hardness.

Suh $[2,66]$ developed a theory that accounts for three components of friction: deformation caused by asperities, plowing, and wear particles remaining in the wear zone. Wear particles in the wear zone may agglomerate, work harden severely, and act as third bodies that deform the contacting surfaces. This model has supporting experimental data for metals that demonstrates that the wear particles contribute to friction.

Earlier theories treat friction, adhesion, and deformation independently. However, current theories treat adhesion and deformation as complimentary to one another. The relative role of the two are still subject to much discussion.

The discussion on friction so far has been limited to macroscopic friction. New experimental techniques such as lateral force microscopy, scanning tunneling microscopy, and the surface force apparatus can probe increasingly smaller surfaces and interfaces. From these techniques a new term was coined: nanotribology, the study of friction and wear at the atomic level. Several models and experiments have sought to explain friction at this scale [72-73]. The laws of macroscopic friction are found to be inapplicable at the microscopic level. For example, at a macroscopic 
level, friction is always accompanied by wear. However, recent experimential studies using lateral force microscopy demonstrate that friction can occur without wear at the atomic level [9].

Experimental studies indicated that friction is strongly influenced by crystal structure, microstructure, temperature, humidity, and compatibility of the sliding surfaces. The limited ductility of ceramics does not allow for the easy formation of adhesive junctions at room temperature. However, ceramics are susceptible to chemical reactions during the test that can change friction drastically. For example, formation of amorphous $\mathrm{SiO}_{2}$ was reported by Fischer et al. [74] for $\mathrm{Si}_{3} \mathrm{~N}_{4}$, sliding in humid air and water mixed hydrocarbons. The low shear strength of this oxide layer resulted in reduction of both friction and wear. Ceramics can also dissipate the mechanical energy created by frictional forces via brittle fracture, in turn increasing friction even more.

\subsubsection{Wear}

Different mechanisms responsible for the material loss during mechanical contact include; adhesion, abrasion, fatigue, oxidation, erosion, and corrosion. This discussion is restricted to adhesion, abrasion, and fatigue. Details of erosive and corrosive wear are discussed in standard books on tribology [1-7].

\subsubsection{Adhesive Wear}

Adhesive wear occurs when the adhesive junctions formed between the asperities of two sliding contacting bodies are stronger than either material. As a result, material transfers from the softer (cohesively weaker) body to the harder (cohesively stronger) body. As the sliding continues, the transferred particles may become loose wear debris and further influence the wear process, through third-body interactions.

After a single pass or a contact, some asperities may deform plastically under the applied load. When the load is removed, these asperities plastically deformed 
under compression, retain residual stresses. These stresses may affect the additional deformation of the asperities by delaying the onset of the yielding. After several passes, it is possible that the deformed asperities carry the load entirely elastically. This process is known as elastic shakedown. At stresses below the shakedown stress, the contact is entirely elastic. Above the shakedown stress, the accumulation of plastic deformation caused by repeated loading can cause fatigue fracture and the generation of wear particles. Thus, fatigue wear proceeds in a sequence of elastic and plastic deformation, work hardening, crack initiation, and crack propagation. Under such repeated loading, cracks can originate at or below the surface. A mathematical expression of wear under such conditions that was derived by Archard [75-76] is given by :

$$
\mathrm{W}=\frac{\mathrm{kF}_{\mathrm{N}}}{\mathrm{H}}
$$

where:

$\mathrm{W}$ is the wear rate (wear volume per unit sliding distance),

$\mathrm{F}_{\mathrm{N}}$ is the normal load,

$\mathrm{H}$ is the hardness, and

$\mathrm{k}$ is the wear coefficient.

The wear coefficient, $\mathrm{k}$, is a measure of severity of wear and has been interpreted as [4] :

- the probability of asperity interaction that produces a wear particle,

- a number that reflects the number of cycles of deformation required to produce a wear particle, and

- the size of the wear particle produced by each contact.

Eqn. [2.4] cannot be used to support or reject any particular mechanism of wear. As explained, the wear coefficient can be interpreted several ways. A wear coefficent of 
$10^{-2}-10^{-3}$ is considered severe wear, whereas a wear coefficent below $10^{-4}-10^{-5}$ is considered mild wear [4]. The size of the wear debris in mild wear is typically $0.01 \mu \mathrm{m}$ to $1 \mu \mathrm{m}$, in contrast to $20 \mu \mathrm{m}$ to $200 \mu \mathrm{m}$ in the case of a severe wear [4]. Acceptable values of the wear coefficient, $\mathrm{k}$, depends on the application.

\subsubsection{Abrasive and Delamination Wear}

Damage to a surface by a harder material is known as abrasive wear. Abrasive wear involves both plastic flow and fracture. Sometimes abrasive wear may occur by plastic flow alone. If wear is caused by the harder surface of the two sliding bodies, the process is known as two-body wear. However, sometimes free particles can be as effective as external abrasive particles. In this case, damage to the surface is said to be caused by three-body wear. The direct proportionality of wear resistance and hardness is also observed in abrasive wear as in adhesive wear. Factors that influence the wear coefficient $\mathrm{k}$ in abrasive wear are:

- the geometry of the abrasive particles,

- the strain hardening of materials,

- the number of particles that deform the surface either by cutting or wedge formation, and

- the volume removed.

Delamination wear typically results from a fatigue-stimulated process. Suh and Jahanmir $[77,78]$ proposed that repeated loading and unloading cycles may induce subsurface cracks that lead to the delamination wear mechanism. These cracks propagate parallel to the surface and eventually extend up to the free surface. Therefore, fatigue should result in plate-like wear particles. Crack formation and propagation in wear are closely associated with fracture toughness. 


\subsubsection{Role of Fracture Toughness}

Fracture toughness is a measure of resistance to crack propagation. Fracture can occur in ductile as well as brittle materials. Microcracking along with other abrasive mechanisms of microploughing and microcutting in a material elucidates a correlation between fracture toughness and abrasive wear resistance. Figure 2.2 shows that abrasive wear resistance of brittle materials initially increases with fracture toughness. For a given testing condition, a linear relationship between wear resistance and fracture toughness is observed below a critical value for fracture toughness. Above this critical fracture toughness, an inverse relationship is observed. The inverse relationship arises when the wear mechanisms are controlled by plastic flow; it is well known that the fracture toughness varies inversely with hardness or yield strength. In summary, there might be a critical value of fracture toughness that initiates the wear process controlled by the crack nucleation, by crack propagation, or by both.

There have been models by several researchers that relate fracture toughness and wear resistance [80-82]. These models have been based on several assumptions about the geometry and the origin of the cracks. All these models predict that wear of brittle materials depends strongly on fracture toughness rather than hardness. Mechanical properties data show that, in general, fracture toughness is inversely related to hardness, as shown in Figure 2.3. In addition, these models also predict that abrasive wear in brittle materials is controlled by nucleation and propagation of cracks above a critical load. The literature involving theories of wear of ductile and brittle materials is vast $[69-71,84-86]$. There is no general model that is universally applicable.

\subsection{Contact Mechanics}

Contact mechanics deals with stresses and deformation that arise when surfaces of two solid bodies are brought into contact. The contact is said to be conforming if the two surfaces fit close together without appreciable deformation. On 


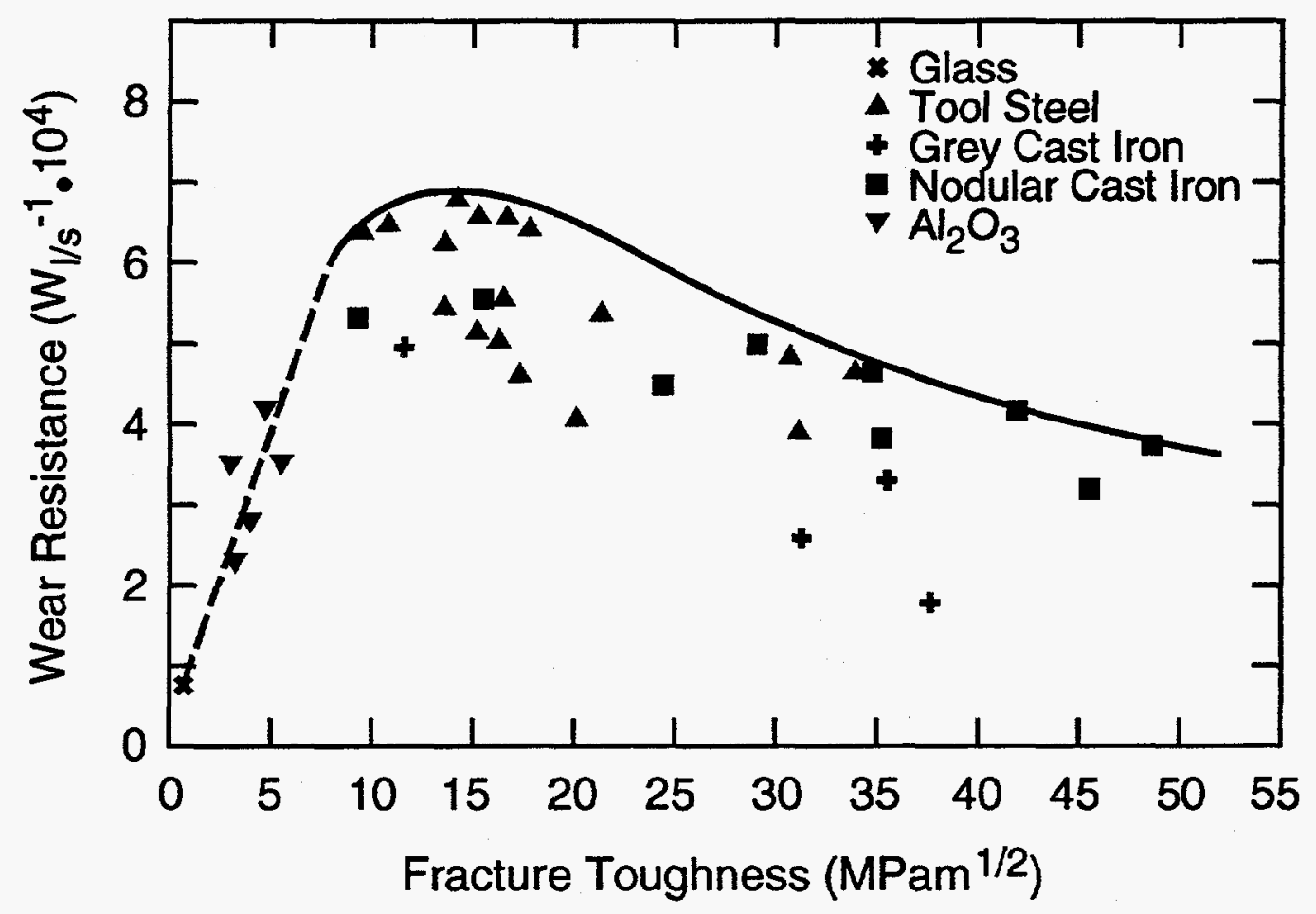

Figure 2.2. Relationship between fracture toughness and abrasive wear resistance from ref. [1]. 


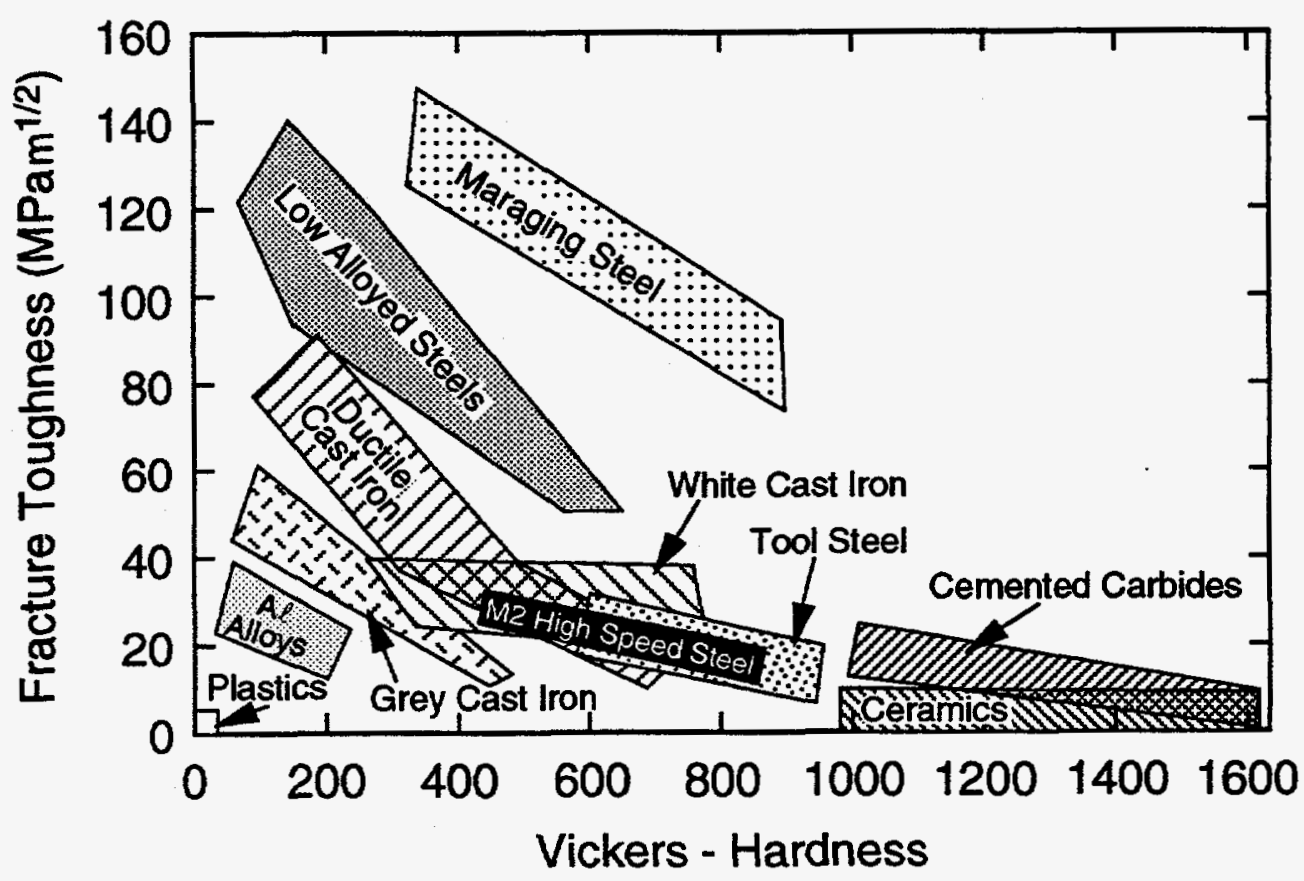

Figure 2.3. Schematic representation of fracture toughness and hardness of plastics, ceramics, and metallic alloys from ref. [1]. 
the other hand, if there is deformation in one of the surfaces, the contact is said to be nonconforming. The two surfaces in a nonconforming contact will have dissimilar pressure profiles. Conforming contacts have large contact area because of their close fit and low contact pressure, whereas nonconforming contacts have small contact area and high contact pressure. Figure 2.4 illustrates conforming and nonconforming contacts.

In most tribological problems, the contacts are nonconforming. Contact stress distributions for different geometries and loads (normal and tangential) contribute significantly to the tribological behavior of a coating. Contact mechanics provides insight into the stress distribution, crack initiation, and crack propagation for surfaces in contact. The use of contact mechanics for industrial problems is complicated because of changes in material properties under working conditions. However, the knowledge of stresses can provide the basis for the first-order design criteria of a coating. In the following section, a brief review of contact mechanics is provided. 


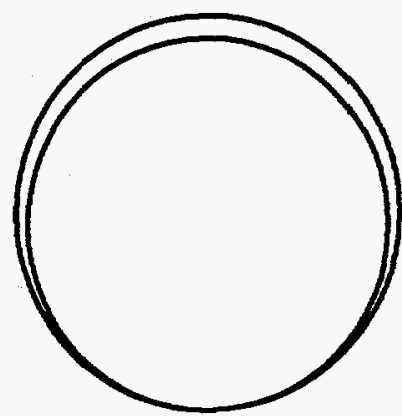

(a)

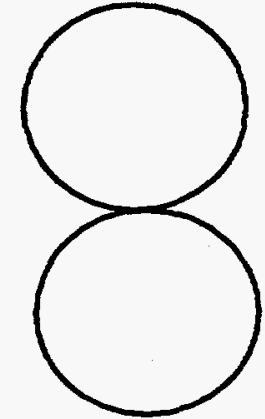

(b)

Figure 2.4. Illustration of (a) conforming and (b) nonconforming contacts. 


\subsubsection{Contact Stress Field Caused by a Spherical Indenter on an Elastic Half-} Space

Hertz derived the expressions for the contact radius and the distribution of pressure for an elastic contact [87]. Results for a sphere loaded elastically against a plane normal to the surface are summarized below. Figures $2.5 \mathrm{a}$ and $2.5 \mathrm{~b}$ show the contact pressure for this geometry without friction and with friction, respectively. Hertz's work was reviewed and extended by Johnson [88]. In the Hertzian limit (that is, the elastic limit), the contact radius for a sphere under normal loading on a plane is given by

$$
a=\left[\frac{3 P R}{4 E_{c}}\right]^{3 / 2}
$$

The variables $P$ and $R$ in Eqn. [2.5] are the load and the radius of the sphere and $E_{c}$ is given by Eqn. [2.2]. The maximum elastic static contact stress for a sphere resting on a plane or a disc is calculated using

$$
P_{\max }=\frac{3}{2} \frac{\mathrm{P}}{\pi \mathrm{a}^{2}}
$$

The contact pressure induces stresses that can lead to deformation in the material close to the contact. Deformation is material-dependent and is responsible for changes in the real area of the contact and the frictional forces. The effect of tangential load on the magnitude of tensile stress at the trailing edge of the sphere is shown in Figure $2.5 \mathrm{~b}$. The symmetry of the maximum contact pressure is disturbed because of the development of a tensile stress at the trailing edge and a compressive stress at the leading edge. The magnitudes of these tensile and compressive stresses increases with increasing friction.

\subsection{2 von Mises Yield Criterion}

Repeated sliding in a tribological contact can lead to severe plastic deformation, fatigue, and eventually crack nucleation at or below the surface. The 

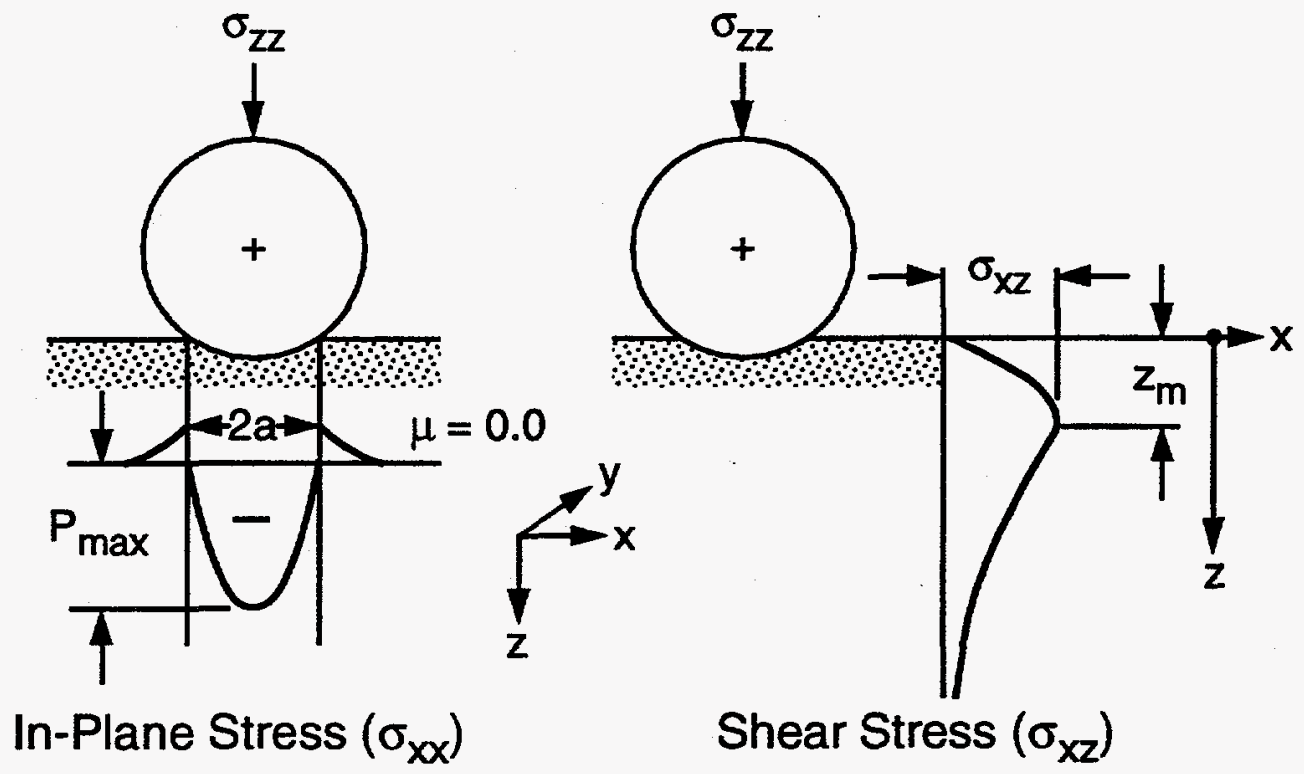

(a)

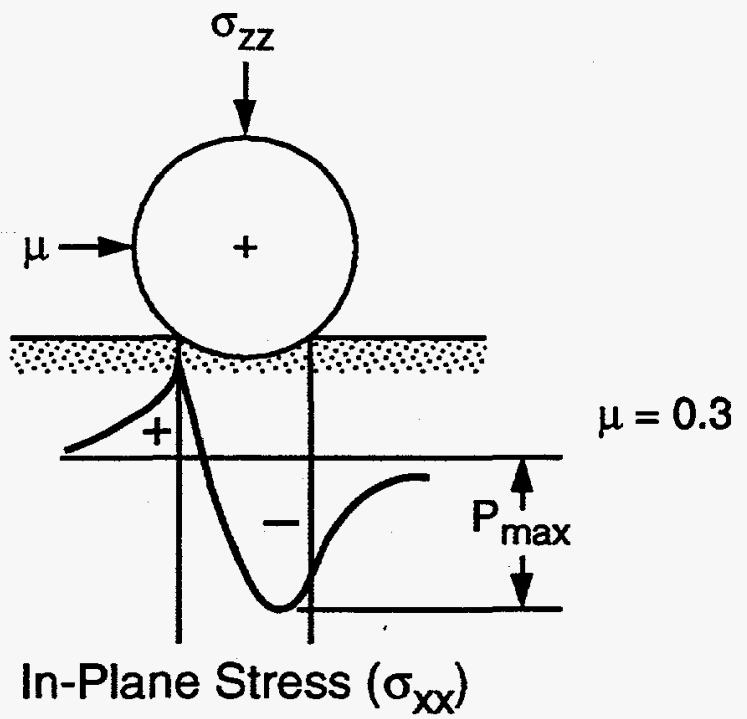

(b)

Figure 2.5. Schematic of the stress distribution for the elastic contact of a sphere and a plane. (a) Normal load. (b) Normal load and tangential load. 
propagation of cracks to the surface results in creation and liberation of wear debris. A flow chart showing the sequence of events in a typical wear process, from loading of the surface to material removal, is shown in the Figure 2.6.

The von Mises stress is accepted as a good criterion for plastic flow, which is the phenomenon that eventually leads for the formation of wear particles. In general, the solids in contact are subjected to a combination of externally applied and internally generated shear and normal stresses. Most materials yield plastically when the shear stress reaches a critical value. The condition for plastic yielding relating to distortion energy is given by the von Mises criterion [89]. The von Mises shear strain-energy criterion is expressed as :

$$
J_{2}=\frac{1}{6}\left[\left(\sigma_{x x}-\sigma_{y y}\right)^{2}+\left(\sigma_{y y}-\sigma_{z z}\right)^{2}+\left(\sigma_{z z}-\sigma_{x x}\right)^{2}\right] \leq \frac{\sigma_{y}^{2}}{3}
$$

where:

$$
\sigma_{\mathrm{xx}}, \sigma_{\mathrm{yy}}, \text { and } \sigma_{\mathrm{zz}} \text { are the principle stresses, and }
$$

$\sigma_{y}$ is the yield strength of the material in uniaxial tension or compression.

When the value of $\left(3 \mathrm{~J}_{2}\right)^{1 / 2}$ exceeds the uniaxial yield strength of the material, $\sigma_{\mathrm{y}}$, yielding occurs.

Hamilton and Goodman $[90,91]$ investigated the stress field of a spherical contact sliding against a rigid plane and used this information to plot the von Mises stresses as a function of depth below the contact. The surface stresses (i.e., at $z=0$ ) in an half-space loaded elastically under plane-strain conditions, where the thickness of the solid is large compared to the width of the loaded region, are summarized in the equations below [ 2.8 to 2.11 ]. Figure $2.5 \mathrm{~b}$ shows the in-plane stress distribution in the elastic half-space of a sliding contact with normal and tangential load. For a 


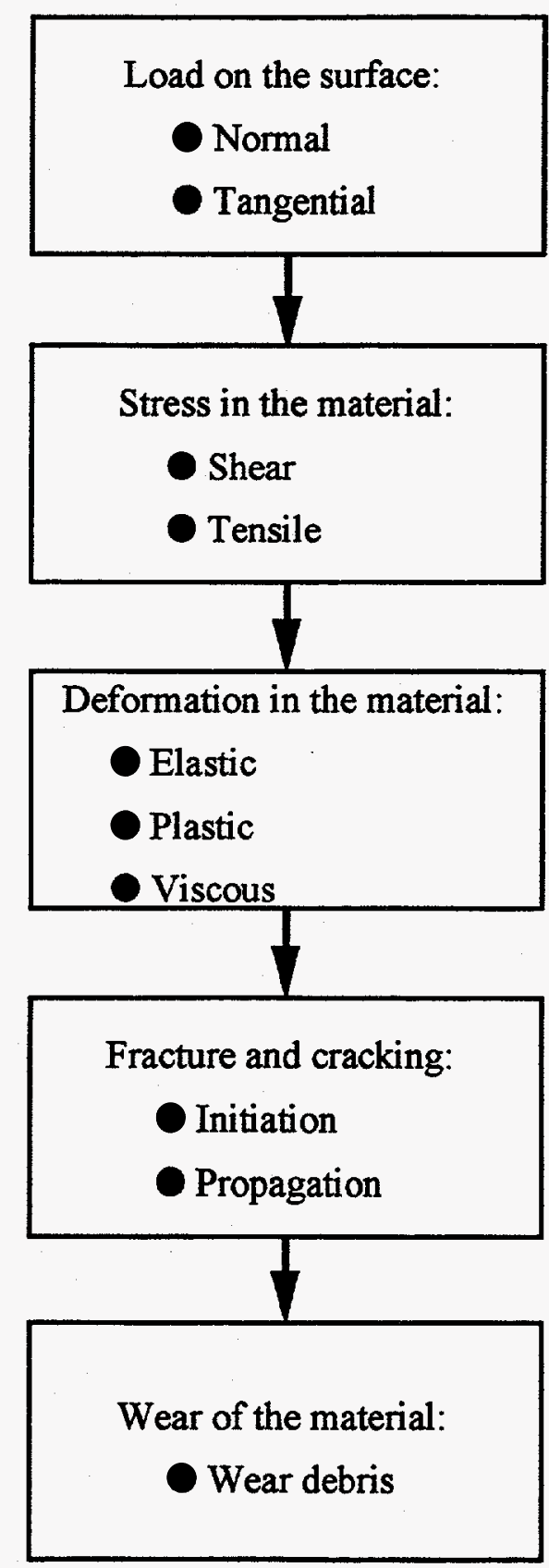

Figure 2.6. Schematic diagram of the process from loading of the surface to wear. 
frictionless contact surface with a normal load $\mathrm{p}$, the shear stress, $\sigma_{\mathrm{x} z}$, and the in-plane normal stress, $\sigma_{\mathrm{xx}}$, are given by

$$
\begin{aligned}
& \sigma_{x z}(x, 0)=0 \\
& \sigma_{x x}(x, 0)=\frac{3 p}{2 \pi a^{3}}\left[-a(1+v)+\frac{a}{2}\right] \quad \text { at } x, y, z=0 \\
& \sigma_{x x}(x, 0)=\frac{3 p}{2 \pi a^{3}}\left[(1-2 v)\left\{x^{2}\right\} \frac{a^{3}}{x^{4}}\right] \quad \text { for } x \geq a \\
& \sigma_{x x}(x, 0)=\frac{3 p}{2 \pi a^{3}}\left[\frac{1}{x^{2}}\left\{\frac{-x^{2}}{x^{2}}\left[\frac{(1-2 v)}{3}\left\{\left(a^{2}-x^{2}\right)^{3 / 2}-a^{3}\right\}\right]-\left(x^{2}\right)\left(a^{2}-x^{2}\right)^{y / 2}\right\}\right]
\end{aligned}
$$

Tangential traction between the two nonconforming bodies with similar elastic constants in a normal contact induces equal and opposite normal displacements at every point on the surface. Thus, the distribution of normal pressure is identical. However, when the elastic constants of two materials are different, the pressure distribution no longer remains identical.

Under combined normal and tangential loading, friction at the contact interface influences the stress field of the contact. When sliding occurs and the coefficient of friction is nonzero, the symmetry of the displacements and the stresses is lost. The effect of tangential traction is to add to the tension on the trailing edge of the contact and the compression on the leading edge as shown in Figure 2.5b. Under these conditions the shear stress, $\sigma_{x x}$, and the in-plane stress, $\sigma_{x x}$, are given by

$$
\begin{array}{ll}
\sigma_{x z}(x, 0)=\frac{3 \mu p}{2 \pi a} & |x| \leq a \\
\sigma_{x z}(x, 0)=0 & |x|>a
\end{array}
$$




$$
\begin{array}{cc}
\sigma_{x x}(x, 0)=\frac{3 \mu p}{2 \pi a^{3}}\left[-x\left(1+\frac{v}{4}\right) \phi+\frac{a x M_{0}}{x^{4}}\left\{-v M_{0}^{2}\left(\frac{3}{2}-\frac{2 x^{2}}{x^{2}}\right)+\frac{7 v x^{2}}{4}-2 v x^{2}+x^{2}\right\}\right] & \text { for } x>a \\
\sigma_{x x}(x, 0) & =\frac{3 \mu p}{2 \pi a^{3}}\left[-\frac{\pi x}{2}\left(1+\frac{v}{4}\right)\right] \quad \text { for } x<a \quad
\end{array}
$$

In Eqn. [2.14], $\mu$ is the friction coefficient, $\phi=\tan ^{1}\left(\frac{a}{M}\right), M=\left(\frac{S+A}{2}\right)^{0.5}, A=x^{2}-a^{2}$, $\mathrm{S}=\mathrm{A}$, and $\mathrm{M}_{0}=\left(\mathrm{x}^{2}-\mathrm{a}^{2}\right)^{0.5}$. The maximum tensile stress on the surface occurs at the trailing edge of the contact $(x=-a)$ and is given by

$$
\sigma_{x x}(-a, 0)=\frac{3 p}{2 \pi a^{2}}\left[\frac{1-2 v}{3}+\frac{4+v}{8} \pi \mu\right]
$$

There are no simple analytical expressions to describe the variation of normal stresses as a function depth. A full description of these stress fields requires computer analysis. Figure 2.7 shows contour plots of the von Mises stress for $\mu=0,0.25$, and 0.5 from Hamilton's and Goodman's [91] work. Their investigation revealed that the maximum value of the von Mises stress occurs beneath the center of the slider for zero friction and low coefficient of friction. As the friction increases, this peak in the contour moves forwards in the direction of sliding and towards the surface.

Equations [2.15] and [2.16] predict the influence of the coefficient of friction on the in-plane stress distribution at the surface and is plotted in Figure 2.8. The highstress tensile region that develops at the trailing edge of the contact is also apparent in Figure 2.8. Tangential traction also produces a compressive stress at the leading edge. Because the trailing edge is in tension, it is more susceptible to crack nucleation. 
(a)
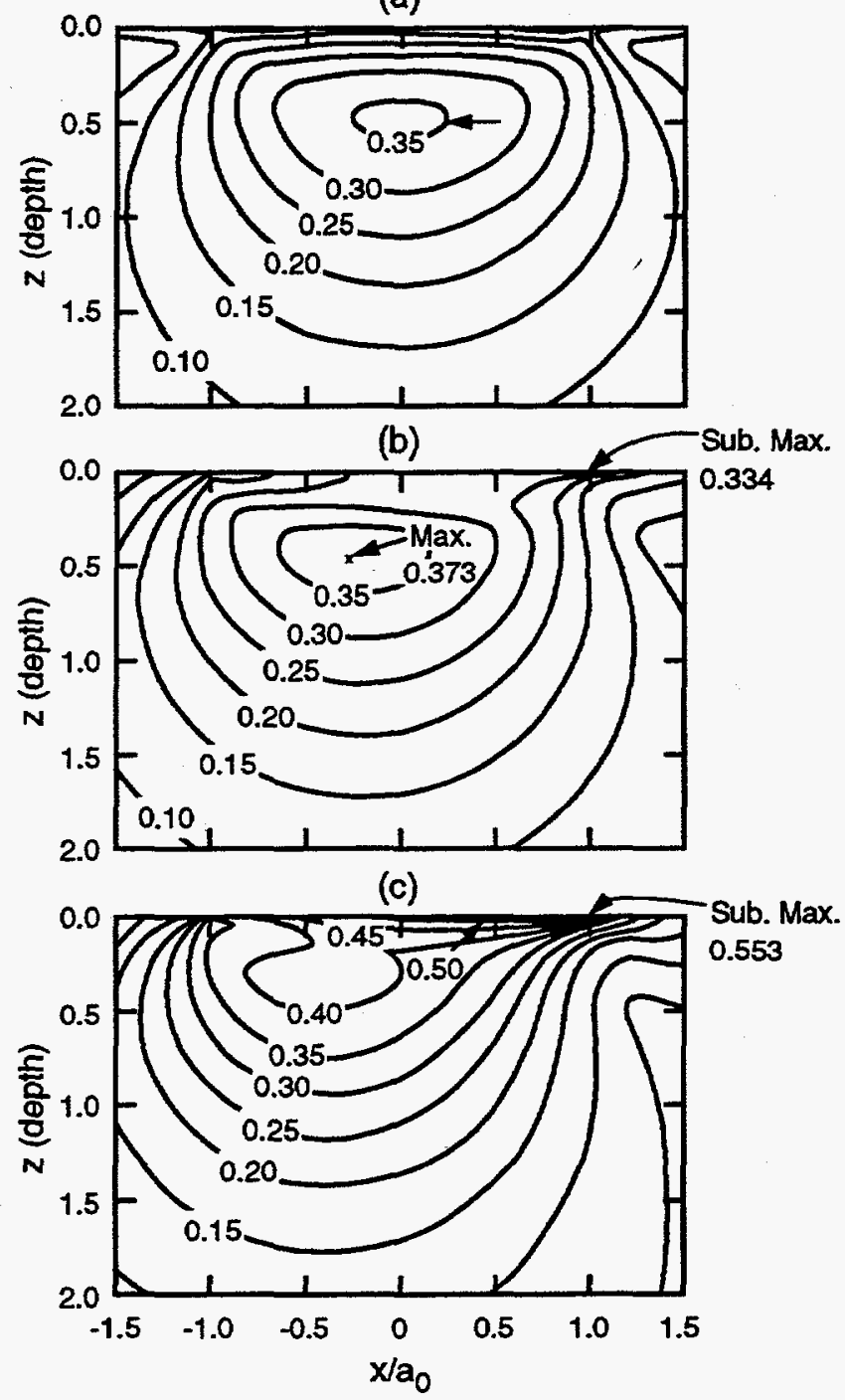

Figure 2.7. Contour plots of the von Misses stress (normalized to $\mathrm{P}_{0}$ ) for the elastic contact of a sphere and a plane:

(a) $\mu=0$, (b) $\mu=0.25$, (c) $\mu=0.5$, from ref. $[90,91]$. 


\subsubsection{Contact Stress Field Caused by a Spherical Indenter on a Layered Elastic Half-Space}

\subsubsection{Normal Load}

Burmister [92] developed the elasticity theory necessary to solve the stress state for the case of a single layer on a bulk substrate for normal contact without sliding. Chen [93] extended this analysis for axisymmetric and nonaxisymmtric normal surface loading. Gupta and Walowait [94] investigated a layered half-space under normal contact with plane-strain. Analytical and finite element solutions for the elastic stress fields produced in thin and relatively stiff layers for the same conditions were considered by van der Zwagg and Field [95], Djabella and Arnell [96], and Komvopolus [97]. Komvopolus [97] also considered the effect of friction at the contact zone. The results for a normal contact from these researchers [95-97] suggested that:

- The ratio of layer thickness to contact width $\mathrm{h} / \mathrm{a}$ has a significant effect on the in-plane stresses at the coating surface and the coating/substrate interface, the maximum shear stress at the coating/substrate interface, and the von Mises equivalent stress.

- Relatively thick stiff layers minimize the stresses in the substrate. On the other hand, if the layer is too thin, stresses in the substrate can be higher than those in the nonlayered case.

- The maximum interfacial shear stress, localized in the case of a thin coating, is spread over a larger area by thick coatings. Komvopolus' [95] results suggest that the effect of the friction coefficient at the contact zone on the stress field is negligible.

\subsubsection{Normal Load plus Tangential Load}

Figures $2.9 \mathrm{a}-\mathrm{b}$ show the various stresses, such as the normal stresses, the inplane stress, and the interfacial stress, for a layered medium under combined normal and tangential loading. Stress analyses of a layered medium under these loading 


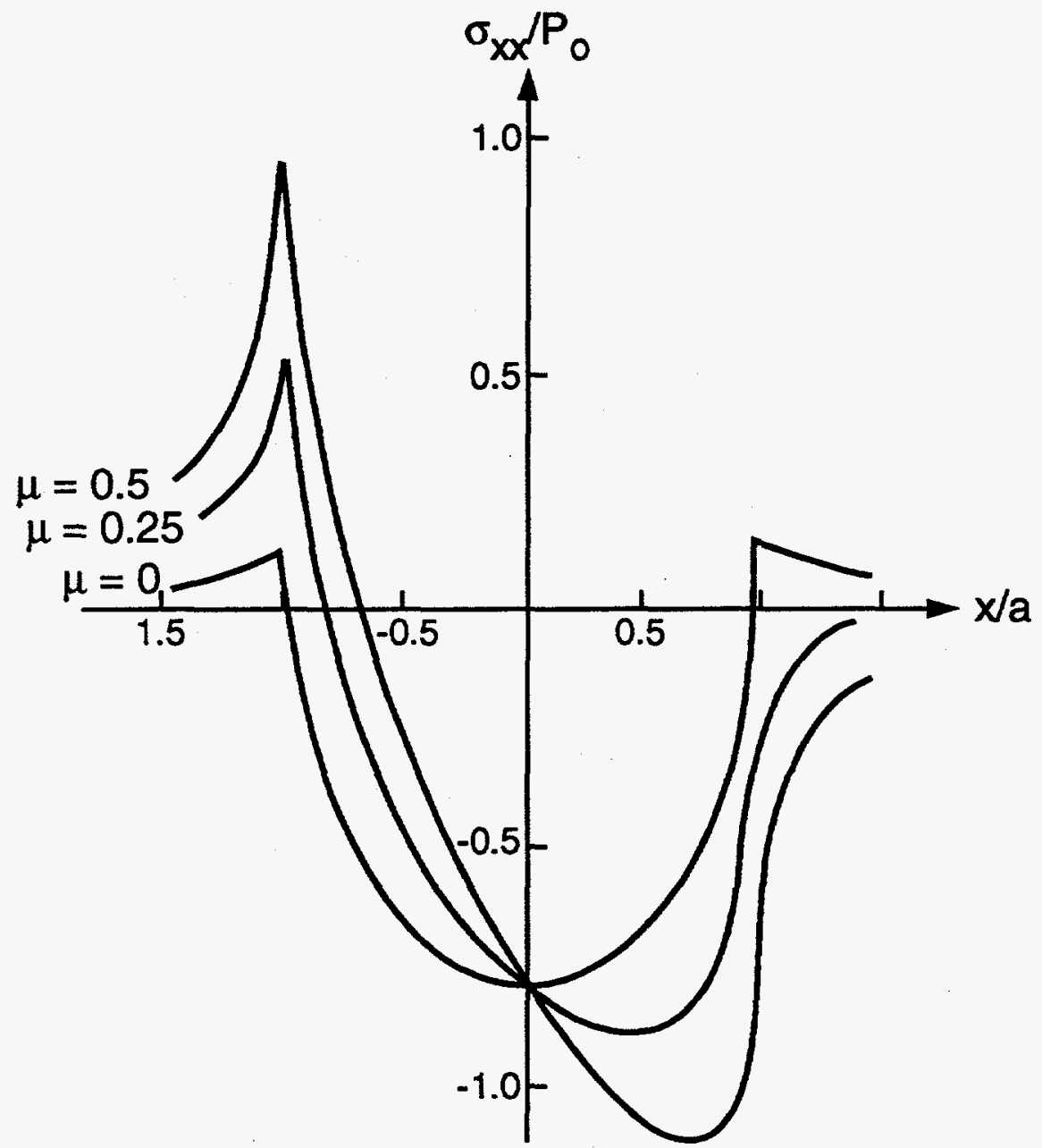

Figure 2.8. Influence of friction on the stress distribution of an elastic contact of a sphere and a plane, from ref. [90, 91]. 
conditions were conducted by Sullivan and King [98, 99], Djabella and Arnell [100], and Leroy and Villechaise [101]. Sullivan and King [98, 99] solved the stresses analytically, whereas Djabella and Arnell [100] and Leroy and Villechaise [101] used finite elemental modeling. The stress state of a single layer system was studied as a function of thickness to contact width ratio $(\mathrm{h} / \mathrm{a})$, coating modulus to substrate modulus ratio $\left(\mathrm{E}_{\mathrm{c}} / \mathrm{E}_{\mathrm{s}}\right)$, and friction $(\mu)$. Table 2.1 summarizes the parameters from these three groups of researchers. Results of their analyses revealed that the modulus of the layer relative to the substrate and coating thickness has a strong influence on:

- the potential for yielding in both the layer and substrate,

- adhesion failure of the substrate-coating interface, and

- crack initiation and propagation caused by in-plane stresses.

Table 2.1 Summary of the parameters used by Sullivan and King [98, 99], Djabella and Arnell [100], and Leroy and Villechaise [101] in solving the contact stress fields of layered elastic half-space.

\begin{tabular}{|c|c|c|c|}
\hline Parameter & $\begin{array}{c}\text { King and Sullivan } \\
{[97,98]}\end{array}$ & $\begin{array}{c}\text { Djabella and Arnell } \\
{[100]}\end{array}$ & $\begin{array}{c}\text { Leroy and } \\
\text { Villechaise [101] }\end{array}$ \\
\hline \hline $\mathrm{h} / \mathrm{a}$ & $0.05-4^{*}$ & $0.1-1.0$ & $0.01-4$ \\
\hline $\mathrm{E}_{\mathrm{c}} / \mathrm{E}_{\mathrm{s}}$ & $0.5,1,2$ & $1,2,3,4$ & $0.5,2,3$ \\
\hline$\mu$ & $0,0.25,0.5$ & $0.15,0.3,0.5$ & $0,0.3,0.5$ \\
\hline & & & \\
\hline
\end{tabular}

The thickness to contact width ratio ( $\mathrm{h} / \mathrm{a})$ and the point where the stresses were calculated in these three studies were different [98-101], which makes the comparison of absolute magnitudes difficult. However, trends for the tensile stress at the surface and the shear stress at the coating-substrate interface can be compared. 


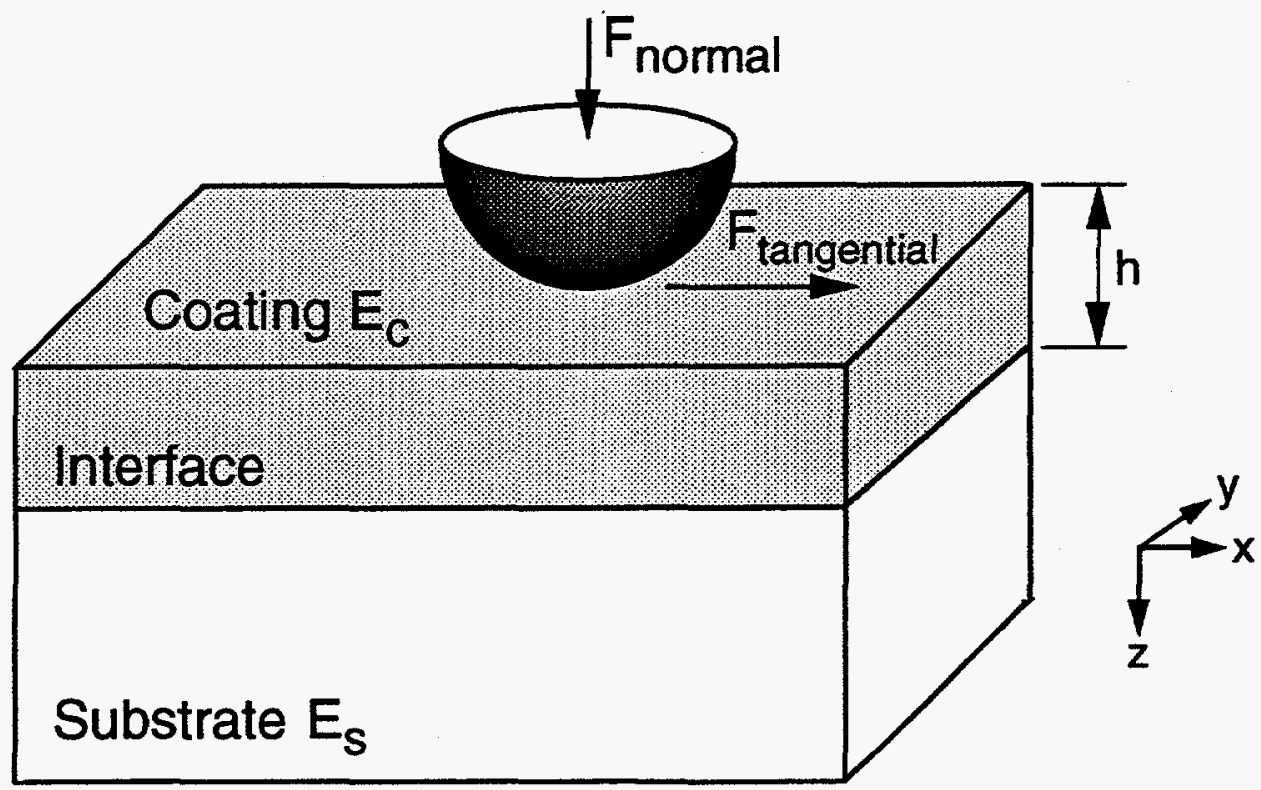

Figure 2.9a. Schematic diagram of an elastic contact of a sphere on a layered medium.

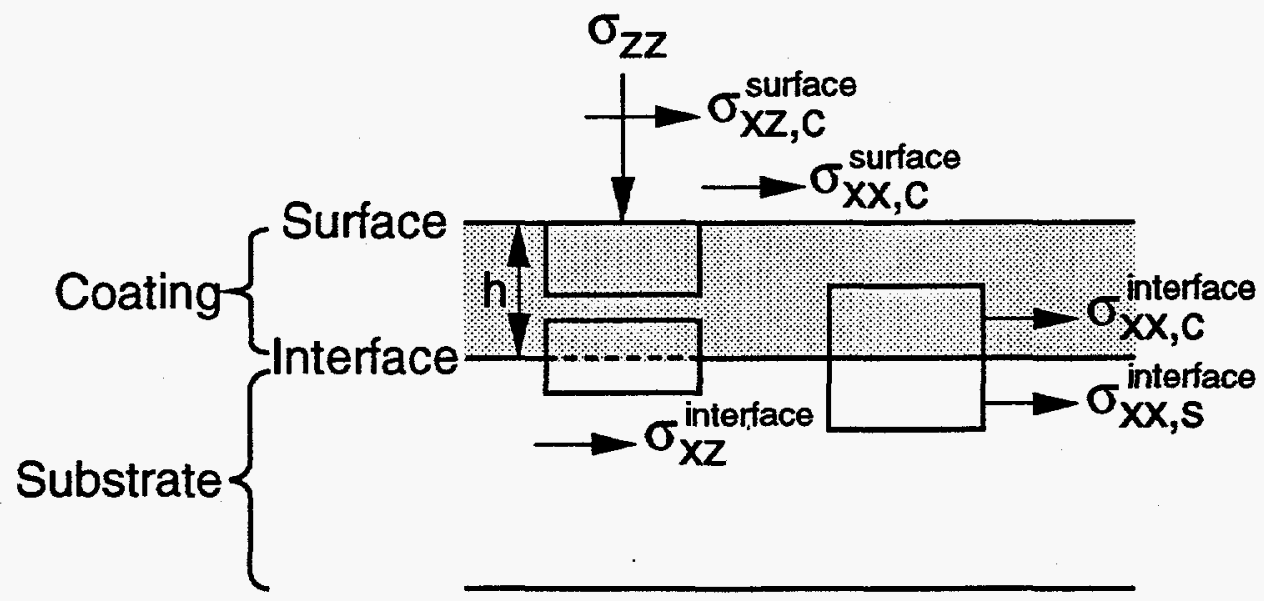

Figure $2.9 \mathrm{~b}$. Illustration of stresses of an elastic contact of a sphere on a layered medium. 
Leroy and Villechaise [101] have shown that for $\mu=0.5$, the in-plane stress at the leading edge, decreased in stiff coatings and increased in compliant coatings, as a function of thickness to contact width ratio. Djabella and Arnell [100] observed similar trends in the in-plane stress, at the leading edge for a coefficient of friction 0.5 . The in-plane stress analysis by King and Sullivan $[98,99]$ for the same value of the friction coefficient $(0.5)$ and at $h / a=1.0$ also indicated that this stress decreases with an increase in modulus. Thus, the results from all three groups [98-101] suggested that for a fixed substrate modulus, the maximum tensile stress at the surface increases significantly with the friction coefficient and the coating modulus. Figure 2.10 shows the normalized in-plane stress, $\sigma_{x x}$, for different ratios of the coating modulus to substrate modulus, and different coefficients of friction from King and Sullivan's work $[98,99]$. This figure shows that at a higher coefficient of friction, the magnitude of tensile stress is low when the modulus of the coating is lower than that of the substrate.

The interfacial shear stress for a given $\mathrm{h} / \mathrm{a}$ and modulus ratio, in general, decreases with increased thickness in both Djabella and Arnell [100] and Leroy and Villechaise's [101] work. In addition, results from both groups suggest that for a given coating thickness, the interfacial shear stress increases with friction. The results of interfacial stresses for three different values of the friction coefficient $(0,0.25$, and 0.5 ) from King and Sullivan's $[98,99]$ work are presented in the Figure 2.11. These results suggest that the interfacial shear stress increases with friction for both the stiff and compliant coatings. The location of the maximum stress shifts towards the surface with increasing friction. This figure also indicates that the magnitude of the maximum interfacial stress for a given friction coefficient increases with modulus ratio. Thus, the trends that were observed in the interfacial stresses by all the three groups are in good agreement. In Figures 2.10 to $2.13, \mathrm{a}_{0}$ and $\mathrm{P}_{0}$ are the contact zone radius and 

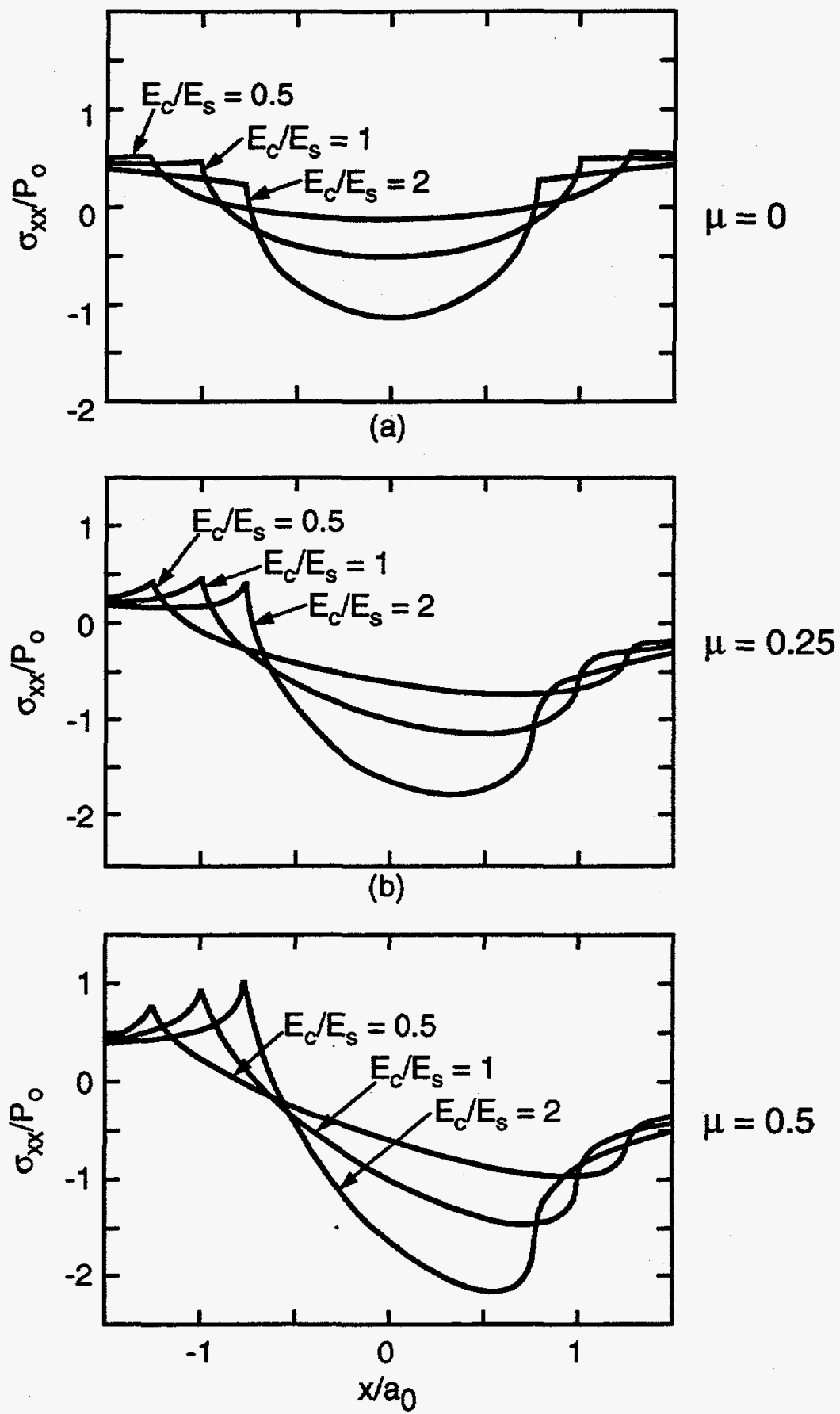

(c)

Figure 2.10. In plane stress distribution on the surface of an elastic contact of a sphere with layered elastic half-space:

(a) $\mu=0$, (b) $\mu=0.25$, (c) $\mu=0.5$ from ref. $[98,99]$. 

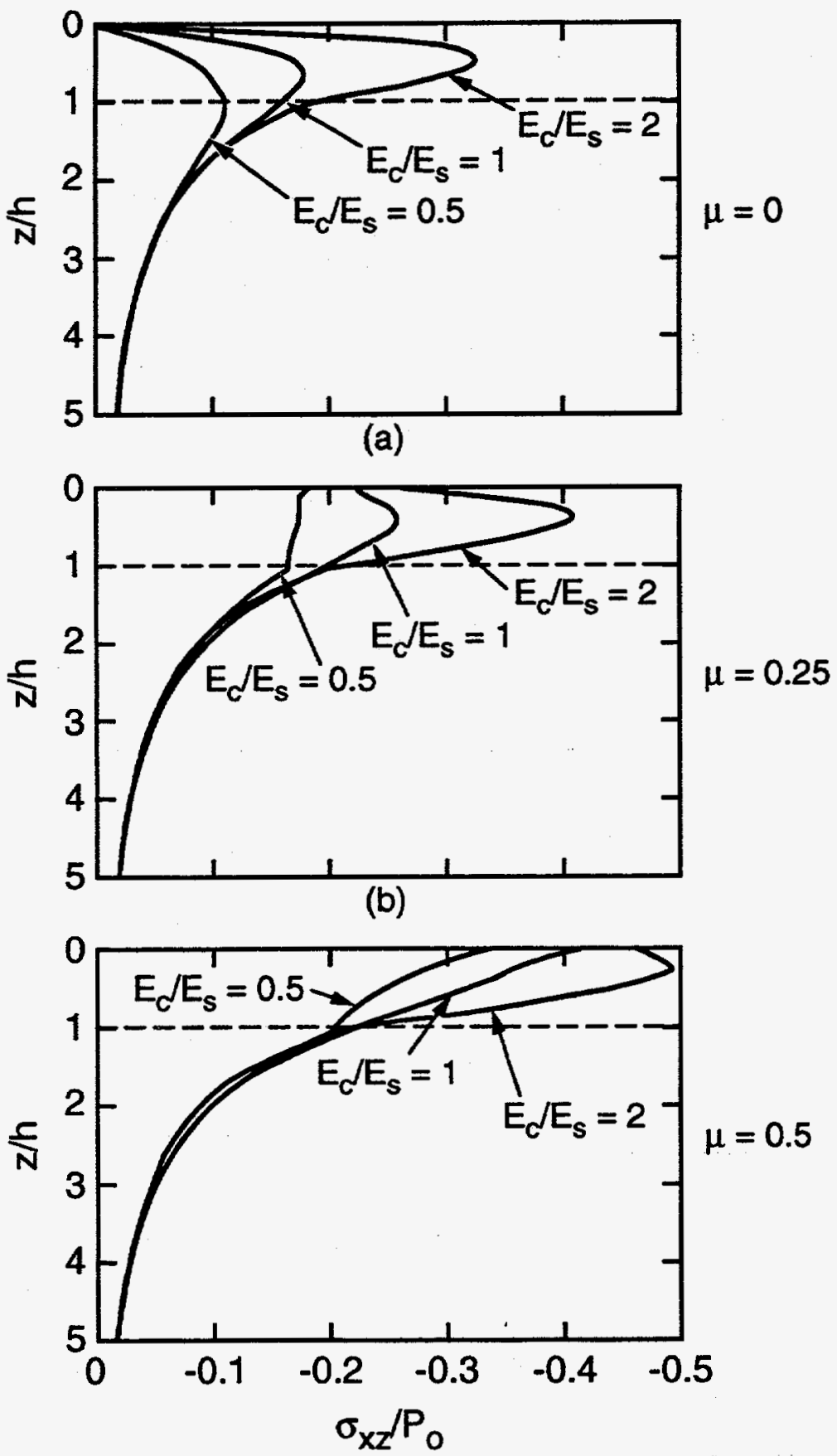

(c)

Figure 2.11. Shear stress distribution on the surface of an elastic contact of a sphere with layered elastic half-space: at $\frac{\mathrm{x}}{\mathrm{a}_{0}}=0.5$, (a) $\mu=0$, (b) $\mu=0.25$, (c) $\mu=0.5$, from ref. $[98,99]$. 
pressure under the spherical indenter for $\mathrm{E}_{\mathrm{c}}=\mathrm{E}_{\mathrm{s}}$, whereas $\mathrm{a}$ is the contact zone radius for $\mathrm{E}_{\mathrm{c}} \neq \mathrm{E}_{\mathrm{s}}$.

Results of the shear stress, $\sigma_{\mathrm{xz}}$, and the normal stress, $\sigma_{\mathrm{zz}}$, and the in-plane stress, $\sigma_{\mathrm{xx}}$ from King and Sullivan's $[98,99]$ work on thin coatings $(\mathrm{h} / \mathrm{a}=0.05)$ for $\mu=0.5$ are shown in the Figure 2.12. In this figure, the stress calculations are presented for $x=a / 2$, where $a$ is the contact radius. These plots indicate that the normal stress and the shear stress do not change significantly with coating modulus (Fig. 2.12a, c). However, the in-plane stress $\sigma_{\mathrm{xx}}$ changes significantly with coating modulus (Fig. 2.12b).

The maxima of the von Mises stress in both stiff and compliant coatings from Leroy and Villechaise's work [101] are approximately double the values of those obtained by King and Sullivan $[98,99]$. On other hand, the stresses agree qualitatively for a stiff coating if comparison is made between Djabella and Arnell [100] and King and Sullivan [98, 99]. Djabella and Arnell [100] did not perform an analysis of soft coatings. All three groups of researchers [98-101] reported that the maximum stress also increases with friction for a given modulus.

The von Mises stress contours for a friction coefficient of 0.25 for three different ratios of the coating to the substrate moduli are shown in Figure 2.13. The von Mises stress is normalized to $\mathrm{P}_{0}$, which is the pressure under the center of the indenter for $\mathrm{E}_{\mathrm{C}}=\mathrm{E}_{\mathrm{S}}$. The trends that were observed in the von Mises stress contours were similar to those observed in bulk materials by Hamilton and Goodman [90, 91]. For example, the magnitude of the maximum tensile stress located at the trailing edge increases with the coefficient of friction. The location of this maximum tensile stress moves towards the surface with the coefficient of friction. In Figure 2.13, the von Mises stresses were compared for the same normal load and the same thickness. The 

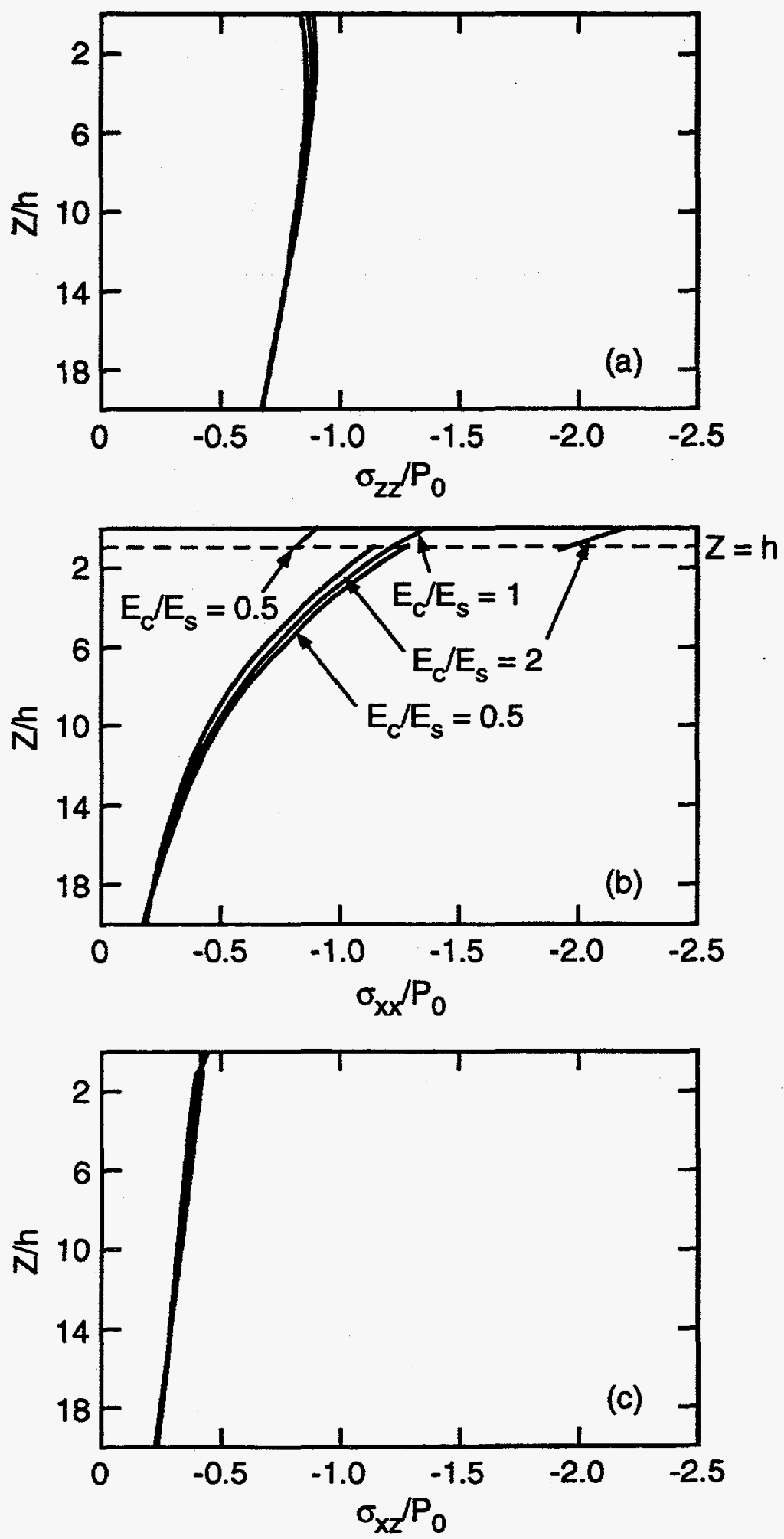

Figure 2.12. Stress distribution at $\mathrm{x} / \mathrm{a}=0.5$, for $\mathrm{h} / \mathrm{a}=0.05$ and $\mu=0.5$ from ref. $[98,99]$. 
(a)

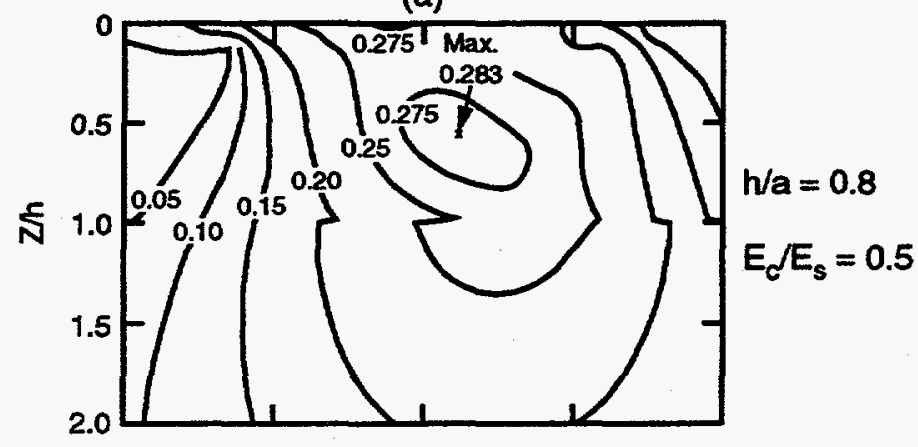

(b)

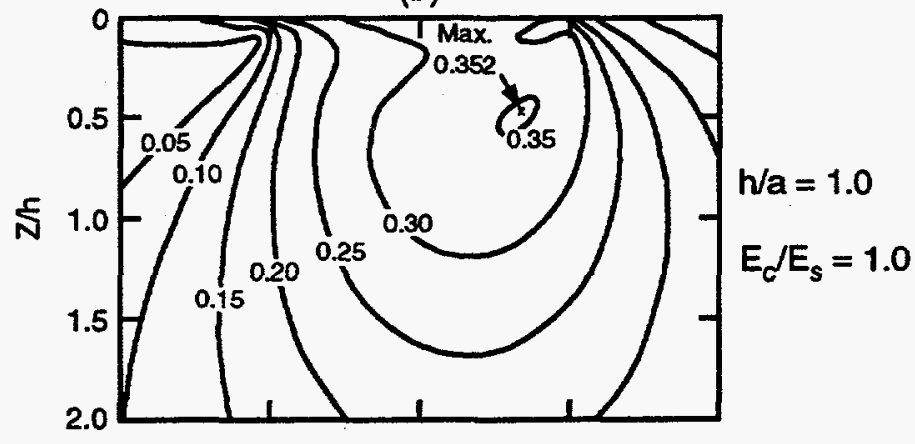

(c)

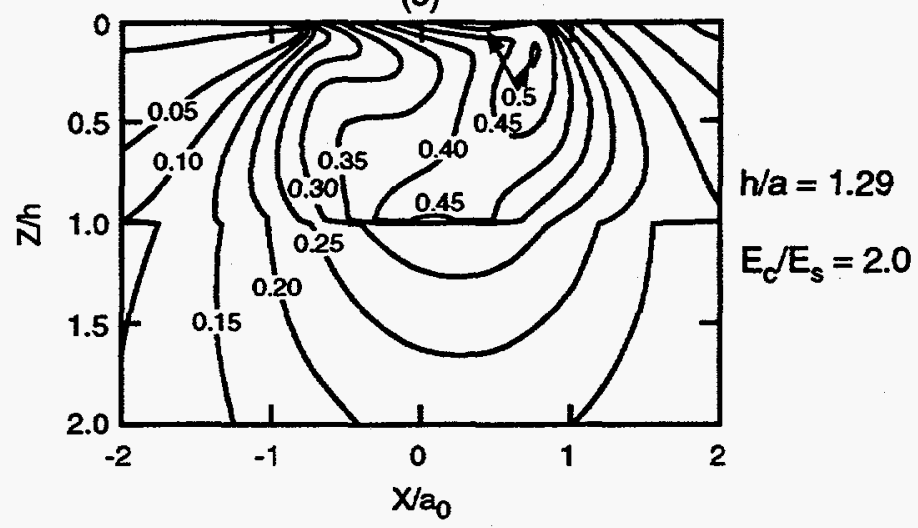

Figure 2.13. Contour plots of the von Misses stress for (normalized to $P_{0}$ ) $\mu=0.25$ for different coating to substrate modulus ratio and under the same loading conditions.

(a) $\mathrm{E}_{\mathrm{c}} / \mathrm{E}_{\mathrm{s}}=0.5$, (b) $\mathrm{E}_{\mathrm{c}} / \mathrm{E}_{\mathrm{s}}=1.0$, (c) $\mathrm{E}_{\mathrm{c}} / \mathrm{E}_{\mathrm{s}}=2.0$ from ref. $[98,99]$. 
von Mises stress contour plots (Figures 2.13a-c) show the change in the location and magnitude of the maximum von Mises stress of the coated case as compared to the uncoated case (i.e., $\mathrm{E}_{\mathrm{C}}=\mathrm{E}_{\mathrm{S}}$ ). These plots also show that the point of maximum von Mises stress moves closer to the surface with an increasing coating modulus and thickness.

Djabella and Amell [102-103] extended the finite elemental modeling to multilayered systems that can minimize the effect of sharp material mismatch on interfacial and shear stresses. They studied the elastic stresses of thin double-layered coatings with $h / a=0.2$ and thick multi-layered coatings with $h / a=1.0$. Their results indicated that for both types of loading, i.e., normal and normal plus tangential loading conditions:

- Tensile stresses at the surface are reduced for relatively thick multilayered coatings consisting of monolayers, double layers, or multilayers as compared to thin double-layered coatings.

- Tensile stresses at the layer-substrate interface are also reduced for a system with multilayers with graded interface as compared to a single or double layer of the same total thickness.

- Residual stresses that are introduced due to processing can lead to instantaneous debonding caused by sharp material mismatch for thick coatings. Multilayered coatings can be synthesized without such a delamination problem.

Thus, the magnitude of the stresses that can cause the failure of the coating is smaller for thick coatings consisting of monolayers, double layers, or multilayers than thin coatings. Hence, coatings with a thick multilayered system will perform better than a thin multilayered system. They also suggest that simultaneous minimization of all the stresses that are responsible to coating failure is not possible.

To summarize, the layer modulus, thickness, and friction coefficient influence the stress distribution of a layered medium under combined normal and tangential 
loading conditions. The complexity involved in a tribological test does not allow the direct correlation of the experimental data with the results from modelling. One of the objectives of this study was to explore the possibility of taking results from contact mechanics in evaluating the tribological performance of surface-modified materials.

\subsection{Introduction to Surface Engineering}

As shown in the previous section, the tribological behavior of a system depends on contact conditions, physical and chemical behavior of the contacting materials, and the thickness, hardness, and elastic modulus of the surface layer. One way to influence the tribological response of a system is to change the properties at the surface that affect the contact mechanics, the hardness, and the toughness of a material.

The surface of an engineering component is important because most failures originate at the surface either by wear, fatigue, or corrosion. Failure at the surface can be prevented or delayed by modifying the surface. A growing area of technology is in the design and implementation of surface engineering processes that can be used to effectively and economically tailor and modify the outermost regions of a solid. Two principal drivers for the evolution of surface engineering are its great flexibility and cost benefits.

When design engineers select materials for an application, they are often constrained by macroscopic and structural property requirements, such as strength and/or modulus-to-weight ratios, and thermal and structural stability. Economic factors such as the cost of materials and fabrication, including machining and casting, are also important. Other properties that influence the performance and lifetime of the material in service, such as surface cleanliness, friction coefficients, and resistance to oxidation, corrosion, and wear, become secondary considerations because in many instances materials with good performance properties in these aspects do not satisfy the structural or economic constraints of the application. The ability to mix harder and 
softer phases in a controlled fashion and to tailor coatings for hardness, fracture toughness, and modulus by surface engineering is both unique and beneficial. Surface engineers choose bulk materials for their structural properties and economic value, and then tailor their surface and near-surface regions to make them suitable for a given application. Through surface engineering, the properties and performance of materials that cost $\$ 2.00$ per pound can be improved so that they meet or exceed the qualities of materials that cost $\$ 10.00$ to $\$ 20.00$ per pound.

A principal application of surface engineering is wear and friction, where it is common practice to engineer a material's surface properties by employing either a surface modification process or by applying a surface coating. In the modification process, the modified zone is an integral part of the bulk material, whereas for surface coatings, the modified zone is a distinct material deposited onto the bulk material.

Improved wear resistance of the modified surface is attributed to the composition and microstructural changes in the near-surface region. Such changes, in turn, affect mechanical properties such as hardness, toughness, and flow strength and ultimately determine the material's application performance. For example, if the material can be made harder, wear by plowing will be minimized. By reducing plowing at the surface, the total frictional force also decreases. Since the frictional force influences surface and subsurface deformation, a hard surface with low friction will also suppress crack formation and propagation. The occurrence of crack formation and subsequent wear debris generation can also be influenced by controlling and tailoring the state of surface residual stresses.

There are several processing routes to producing modified surfaces for surface engineering applications. The choice of a particular process depends on factors such as the effect of a process on the substrate, the interaction of the modified zone with the substrate, the role or engineering application of the modified zone, and cost effectiveness. Properties of the coating, such as roughness, thickness, Young's 
modulus, yield strength, and shear strength with respect to the substrate, are also important considerations.

Ion beam-based technologies, such as ion implantation and ion beam-assisted or energetic deposition techniques are favored methods for implementing surface engineering. Ion implantation produces modified surfaces that are well-integrated with the substrate, while ion-assisted deposition provides the opportunity of producing thicker modified zones through the build up of a coating that possesses many of the same properties found in ion-implanted surfaces. Table 2.2 lists some examples of materials that have been successfully modified by ion implantation. It can be seen that ion implantation significantly reduced wear for many cases. For example, the friction of nitrogen implanted Ti6Al4V against ruby decreased by factor of 3 , and the wear volume decreased by a factor of 300 .

This dissertation is concerned with the characterization and evaluation of coatings that were synthesized by three surface-engineering methods; namely, ion implantation, plasma-source ion implantation, and DC magnetron sputtering. The details of these surface engineering and characterization techniques are discussed in the next chapter. 
Table 2.2 Tribological properties of ion-implanted surfaces.

\begin{tabular}{|c|c|c|c|c|c|c|}
\hline $\begin{array}{c}\text { Host } \\
\text { Material }\end{array}$ & $\begin{array}{l}\text { Implant } \\
\text { Species }\end{array}$ & $\begin{array}{l}\text { Slider, } \\
\text { Load }\end{array}$ & $\begin{array}{l}\text { Friction Coeffic } \\
\text { Unimplanted }\end{array}$ & Implanted & $\begin{array}{l}\text { Reduction factor } \\
\text { of wear volume* }\end{array}$ & Ref. \\
\hline Pure iron & $\mathrm{N}^{+}$ & $\begin{array}{l}\text { Ruby, } \\
1-2 \mathrm{~N}\end{array}$ & 0.2 & 0.26 & $1-2$ & [17] \\
\hline Ti6Al4V & $\mathrm{N}^{+}$ & $\begin{array}{l}\text { Ruby } \\
2-3 N\end{array}$ & 0.48 & 0.15 & $300-500$ & {$[17,18]$} \\
\hline$\overline{\mathrm{SiC}}$ & $\overline{\mathrm{Ar}^{+}}$ & $\begin{array}{c}\text { Steel }(<1 \% \mathrm{C}) \\
9 \mathrm{~N}\end{array}$ & 0.6 & $\overline{0.2}$ & 5 & [19] \\
\hline $\begin{array}{l}\mathrm{Al}_{2} \mathrm{O}_{3} \\
\text { Polycrystalline }\end{array}$ & $\mathrm{Ti}^{+}$ & $\begin{array}{l}\mathrm{Al}_{2} \mathrm{O}_{3} \\
10 \mathrm{~N}\end{array}$ & 0.45 & $0.26-0.38$ & 10 & [20] \\
\hline WC-Co & $\overline{\mathrm{N}^{+}}$ & $\begin{array}{l}\text { Steel } \\
100 \mathrm{~N}\end{array}$ & 0.22 & 0.11 & $8-9$ & [21] \\
\hline Polyimide & $\overline{\mathbf{N}^{+}}$ & SUJ2 Steel & 0.5 & 0.35 & 4 & {$[22]$} \\
\hline
\end{tabular}




\section{Chapter 3 Experimental Techniques}

A range of experimental techniques were used to perform this research. This chapter summarizes those surface modification techniques. Experimental setups that were used for characterizing various properties are also described.

\subsection{Surface Engineering Methods}

Surface engineering is branch of engineering that deals with tailoring surface properties to improve their function and serviceability, and was described in Section 2.5. In this section, details of the ion-beam based techniques such as beam-line ion implantation (BII), plasma-source ion implantation (PSII), and DC magnetron sputtering are examined. For a detailed discussion of surface engineering, refer to the text on surface engineering [104]. Extensive details of ion solid interactions can be found in the book by Nastasi et al. [16].

\subsubsection{Ion Implantation}

Ion implantation is a process by which virtually any element can be injected into the near-surface region of any solid. When energetic ions penetrate a target or host material, they lose energy through electronic and nuclear collisions. The contribution of the energy loss caused by electronic collisions is dominant if the ion implantation is carried out by using low-mass ions of high energies. On other hand, the contribution of the energy loss caused by nuclear collisions is dominant in an implantation process if high-mass ions of low energy are employed. Before coming to rest, the ions collide with lattice atoms in their path. Sometimes the energy transferred during this collision is sufficient to displace atoms from their lattice sites. The displaced atoms undergo further collisions with other lattice atoms before coming to rest. This multiplication process is known as collision cascade. The actual total distance that ions travel before coming to rest is known as the range of ions, $R$. Since 
energy is lost by the incident ions to target atoms and electrons in a series of discrete collisions, the stopping process is random. This leads to the total distribution of the ions to a near-Gaussian profile. The projected range of ions, $R_{p}$, is the distance that ions travel in the direction of the incident ion beams and perpendicular to the surface of the target. Thus, the mean projected range gives the probable location for an ion to come to rest. The associated spread of the projected range, $\Delta \mathrm{R}_{\mathrm{p}}$, is called straggling. The range, $R$, projected range, $R_{p}$, and straggling, $\Delta R_{p}$ are illustrated in the Figures 3.1a-b.

The nonequilibrium process of ion implantation can invariably lead to metastable structures, super-saturated solutions, amorphous phases, and new alloys. The collision cascade creates a distribution of vacancies, interstitial atoms, and other types of lattice disorder in the region around the ion track. Energy lost by the incident energetic ions on the surface of a solid can sometimes lead to an ejection of target atoms from the surface. This process is known as sputtering. The sputtering process influences the retained dose and surface morphology. The number of foreign atoms in the substrate can be calculated from an assumed Gaussian distribution of the implanted profile (Figure 3.1b). From a surface engineering point of view, the important parameters in the process of ion implantation are ion energy, mass, dose of the ions, and the implantation temperature. All these parameters together dictate the width, composition, and microstructure of the modified region.

The distribution of implanted ions, $\mathrm{N}(\mathrm{x})$, is given by

$$
N(x)=\frac{\phi_{i}}{\Delta R_{p}(2 \pi)^{1 / 2}} \exp \left[-\frac{1}{2}\left(\frac{x-R_{p}}{\Delta R_{p}}\right)^{2}\right]
$$




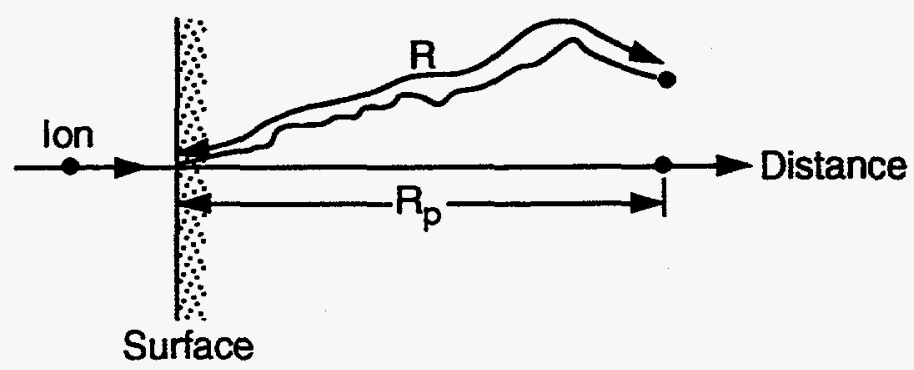

Figure 3.1a. Illustration of range, $R$, and projected range, $R_{p}$, of an incident ion.

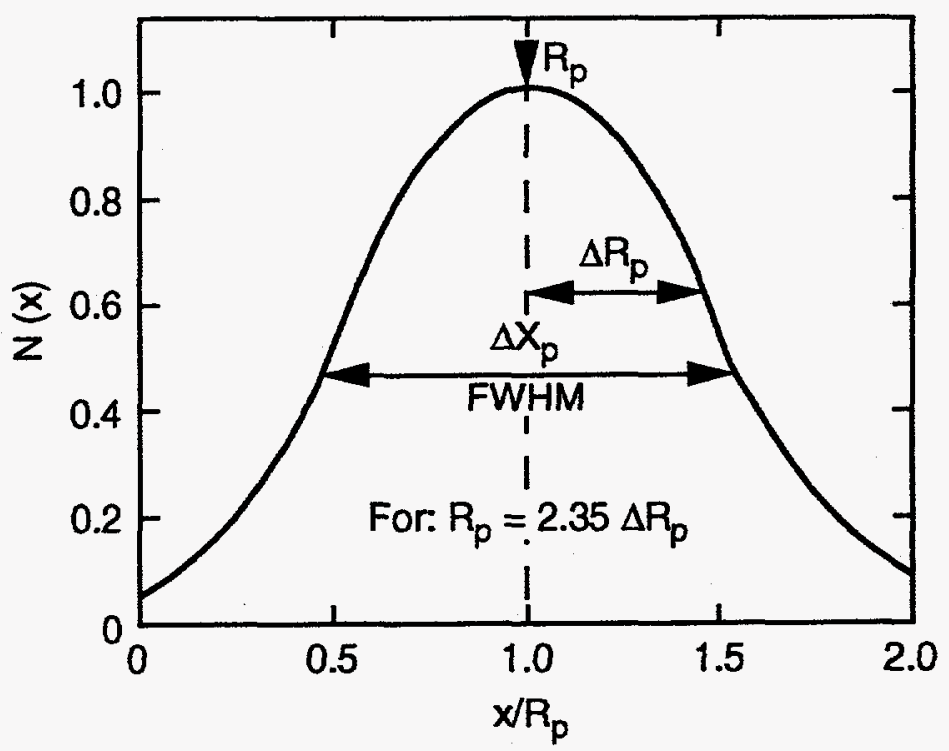

Figure 3.1b. Illustration of Gaussian profile of an implantation process. 
where:

$\phi_{\mathrm{i}}$ is the ion implantation dose,

$\mathrm{R}_{\mathrm{p}}$ is the projected range, and

$\Delta R_{p}$ is the projected range straggling.

The peak atomic density of the implanted species, $N_{p}$, can be obtained by setting $x=$ $\mathbf{R}_{\mathrm{p}}$, in Eqn [3.1]:

$$
N_{p}=\frac{0.4 \phi_{i}}{\Delta R_{p}}
$$

Once the atomic density of the substrate is known, the peak atomic concentration $\mathrm{C}_{\mathrm{p}}$, can be obtained by using

$$
C_{p}=\frac{N_{p}}{N_{p}+N}
$$

A number of Monte Carlo codes have been developed to calculate the range and damage distribution of ions in amorphous materials. Transport of Ions in Matter (TRIM) [105] is the most commonly used computer program. More discussion on this approach is presented in Chapter 4.

The analytical approach used to obtain range quantities was pioneered by $\mathrm{J}$. Lindhard, M. Scaharff, and H. E. Schiott [106] and is commonly known as LSS theory, which can be used to calculate range values with an accuracy of about $20 \%$. In this approach, the total energy-loss rate along the path of the projectile, which includes nuclear and electronic components, determines the range of an ion. This total energy loss can be expressed in terms of the dimensionless units reduced energy, $\varepsilon$, and the range, $\rho_{\mathrm{L}}$. Dimensionless parameters $\varepsilon$ and $\rho_{\mathrm{L}}$ are helpful to establish universal range curves for reduced energy, $\varepsilon$, and the electronic stopping parameter, $\mathrm{k}$. The reduced range, $\rho_{L}$, for Thomas-Fermi nuclear stopping is given by 


$$
\rho_{\mathrm{L}}(\varepsilon)=\int_{0}^{\varepsilon} \frac{\mathrm{d} \varepsilon}{\mathrm{S}(\varepsilon)}
$$

where:

$$
\mathrm{S}(\varepsilon)=\mathrm{S}_{\mathrm{n}}(\varepsilon)+\mathrm{S}_{\mathrm{e}}(\varepsilon),
$$

$\varepsilon$ is the energy of the particle,

$S(\varepsilon)$ is the reduced stopping factor, and

$S_{n}(\varepsilon)$ is the nuclear stopping factor.

$$
\begin{aligned}
& \mathrm{S}_{\mathrm{n}}(\varepsilon)=\frac{3.441 \varepsilon^{1 / 2} \ln (\varepsilon+2.718)}{1+6.335 \varepsilon^{1 / 2}+\varepsilon\left(6.882 \varepsilon^{1 / 2}-1.708\right)} \\
& \varepsilon=\frac{0.03255 \mathrm{M}_{1}}{\mathrm{Z}_{1} \mathrm{Z}_{2}\left(\mathrm{Z}_{1}^{2 / 3}+\mathrm{Z}_{2}^{2 / 3}\right)^{1 / 2}\left(\mathrm{M}_{1}+\mathrm{M}_{2}\right)} \\
& \mathrm{S}_{\mathrm{e}}(\varepsilon) \text { is the electronic stopping factor } \\
& \mathrm{S}_{\mathrm{e}}(\varepsilon)=\mathrm{k} \varepsilon^{1 / 2}
\end{aligned}
$$

where:

$\mathrm{k}$ is the electronic stopping factor

$Z_{1}$ is the atomic number of the incident particle,

$Z_{2}$ is the atomic number of the target atom,

$M_{1}$ is the mass of the incident particle, and

$\mathrm{M}_{2}$ is the mass of the target atom.

$$
\mathrm{k}=\frac{\mathrm{Z}_{1}^{2 / 3} \mathrm{Z}_{2}^{1 / 2}\left(1+\frac{\mathrm{M}_{2}}{\mathrm{M}_{1}}\right)^{3 / 2}}{12.6\left(\mathrm{Z}_{1}^{2 / 3}+\mathrm{Z}_{2}^{2 / 3}\right)^{3 / 4} \mathrm{M}_{2}^{1 / 2}}
$$

Physically, $\varepsilon$ is a measure of how energetic the collision is and how close the incident ions get to the nucleus of the target material. The reduced range, $\rho_{L}$, is given by 


$$
\rho_{\mathrm{L}}=\mathrm{RNM}_{2} 4 \pi \mathrm{a}_{\mathrm{TF}}^{2} \frac{\mathrm{M}_{1}}{\left(\mathrm{M}_{1}+\mathrm{M}_{2}\right)^{2}}
$$

where:

$\mathrm{R}$ is the range of the ions, and

$\mathrm{N}$ is the atomic density.

$\mathrm{a}_{\mathrm{TF}}$ is theThomas-Fermi Screening length $=\frac{0.04692}{\left(\mathrm{Z}_{1}^{2 / 3}+\mathrm{Z}_{1}^{2 / 3}\right)^{1 / 2}}$

Table 3.1 gives the values of reduced range for the different values of reduced energies, $\varepsilon$, and the stopping factor, $\mathrm{k}$, from ref. [107]. Once the reduced energy $\varepsilon$ and the stopping factor $k$, is known, the reduced range, $\rho_{L}$, can be obtained from Table 3.1 . Equation [3.8] can be used to estimate the range of the ions. However, in many surface modification techniques, the projected range of ions, $R_{p}$, is the quantity of interest. Based on LSS theory [106], Winterbon et al. [108] calculated a more exact relationship between projected range of ions, $R_{p}$, and the straggling, $\Delta R_{p}$, for mass conditions $0.1 \leq \frac{M_{2}}{M_{1}} \leq 10$, which is shown in Figure 3.1c. After estimating the range from Eqn. 3.8, the projected range of ions, $R_{p}$, and the straggling, $\Delta R_{p}$, can be calculated from Figure 3.1c.

A schematic diagram of an implanter is shown in Figure 3.1d. The typical ion implanter consists of an ion source to generate ions, an ion extractor to extract charged ions, a mass analyzer to select the desired ions, beam optics to achieve the beam shape and current density, and a target chamber where the substrate is mounted.

The ion implantation process has several advantages. The modified layer is part of the bulk material. The interface between substrate and coating is graded. It is highly controllable and reproducible. Bulk properties and the dimensions of the substrate can be retained. Solid solubility can be exceeded. 
Ion implantation is a low-temperature process, which is both highly controllable and reproducible. Problems concerning adhesion of the modified surface to the bulk are completely eliminated in this process. Bulk properties of the material can be retained. Novel materials that are impossible for thermodynamics reasons can be produced by exceeding solid solubility. Bulk and surface properties can be optimized independently.

Ion implantation processes have been widely used to improve tribological properties of metal, ceramics, and polymers [17-22]. However, this method has some limitations. This process is expensive and is a line-of-sight process. When oddshaped objects are to be implanted, the process becomes complex. However, some limitations of the beam-line implantation have been overcome by the plasma-source ion implantation that has been developed in recent years [27-32].

\subsubsection{Plasma-Source Ion Implantation}

Plasma-source ion implantation (PSII), also known as plasma immersion implantation, has been developed to implant targets of different shapes with ions of gaseous species. The schematic diagram of the PSII implanter is shown in Figure 3.2. It consists of plasma generated either by an RF or a DC glow discharge, the substrate holder, and a power supply for pulse-biasing the substrate. The substrate is immersed in a plasma and is negatively pulse-biased. The plasma ions are accelerated by the electric potential and are implanted into the substrate.

Depending on the amplitude of the biased voltage on the substrate, the ions may be either be implanted or deposited. If the biased voltage is greater than $2 \mathrm{kV}$, the probability of implanting into substrate is greater. Because the substrate is immersed in the plasma, the ions are accelerated from all directions simultaneously at normal incidence, and the shape of the substrate is not a constraint. This conformal implantation eliminates the need for target manipulation. In the PSII process, there are no particle accelerators and mass analyzers. Thus, both atomic and molecular ions are 
Table $3.1 \rho$ Values as a function of $\varepsilon$ and $k$, from ref. [107].

\begin{tabular}{|l|l|l|l|l|l|l|l|l|}
\hline$\varepsilon$ & $\mathrm{k}=0.0$ & $\mathrm{k}=0.1$ & $\mathrm{k}=0.12$ & $\mathrm{k}=0.14$ & $\mathrm{k}=0.2$ & $\mathrm{k}=0.3$ & $\mathrm{k}=0.4$ & $\mathrm{k}=1.0$ \\
\hline 0.01 & 0.072 & 0.069 & 0.069 & 0.068 & 0.067 & 0.064 & 0.062 & 0.052 \\
\hline 0.02 & 0.115 & 0.110 & 0.109 & 0.108 & 0.106 & 0.102 & 0.098 & 0.081 \\
\hline 0.05 & 0.218 & 0.207 & 0.205 & 0.203 & 0.197 & .0188 & 0.180 & 0.144 \\
\hline 0.10 & 0.360 & 0.339 & 0.335 & 0.332 & 0.321 & 0.304 & 0.289 & 0.224 \\
\hline 0.20 & 0.614 & 0.571 & 0.565 & .0553 & 0.533 & 0.501 & 0.472 & 0.353 \\
\hline 0.50 & 1.35 & 1.21 & 1.19 & 1.17 & 1.10 & 1.01 & 0.938 & 0.656 \\
\hline 1.0 & 2.67 & 2.29 & 2.22 & 2.17 & 2.01 & 1.80 & 1.63 & 1.06 \\
\hline 2.0 & 5.84 & 4.57 & 4.39 & 4.22 & 3.79 & 3.26 & 2.88 & 1.71 \\
\hline 5.0 & 19.4 & 11.9 & 11.1 & 10.4 & 8.83 & 7.11 & 5.99 & 3.17 \\
\hline 10.0 & 53.6 & 23.8 & 21.6 & 19.9 & 16.1 & 12.3 & 10.1 & 4.92 \\
\hline
\end{tabular}




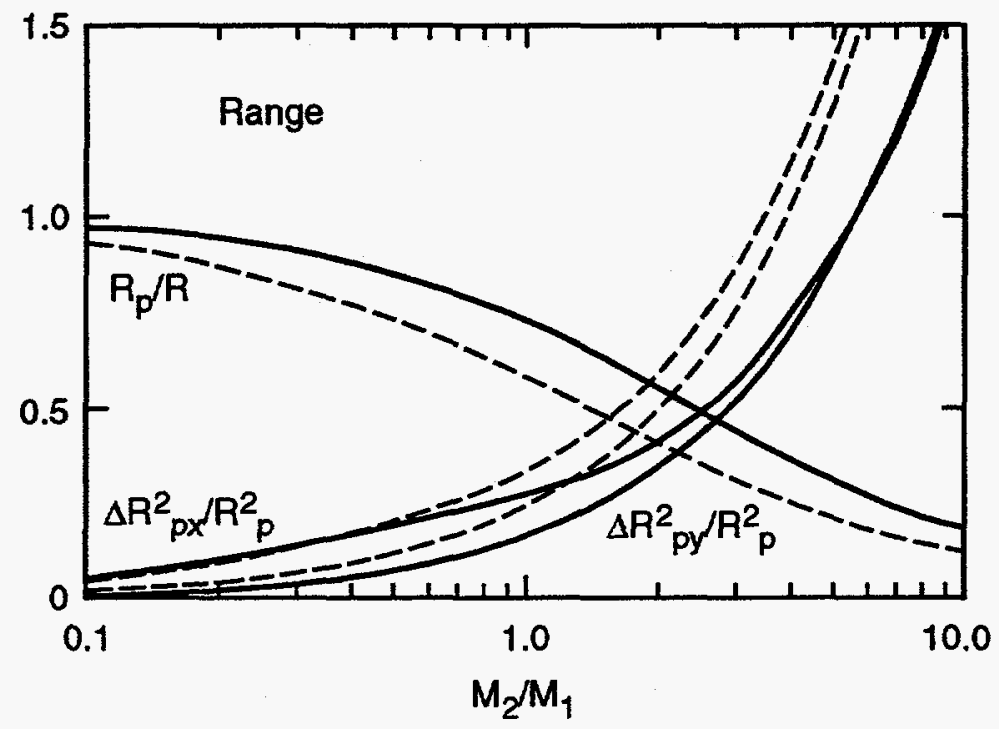

Figure 3.1c. Relationship between the range, $R$, the projected range, $R_{p}$, and the range straggling, $\Delta R_{p}$, as functions of mass ratio, $\mathrm{M}_{2} / \mathrm{M}_{1} . \Delta \mathrm{R}_{\mathrm{px}}$ is in the direction of the incident ion, and $\Delta R_{p y}$ is in the perpendicular direction of the incident ion. Dashed lines, $m=1 / 3$, solid lines, $m=1 / 2$, from ref. [16]. 


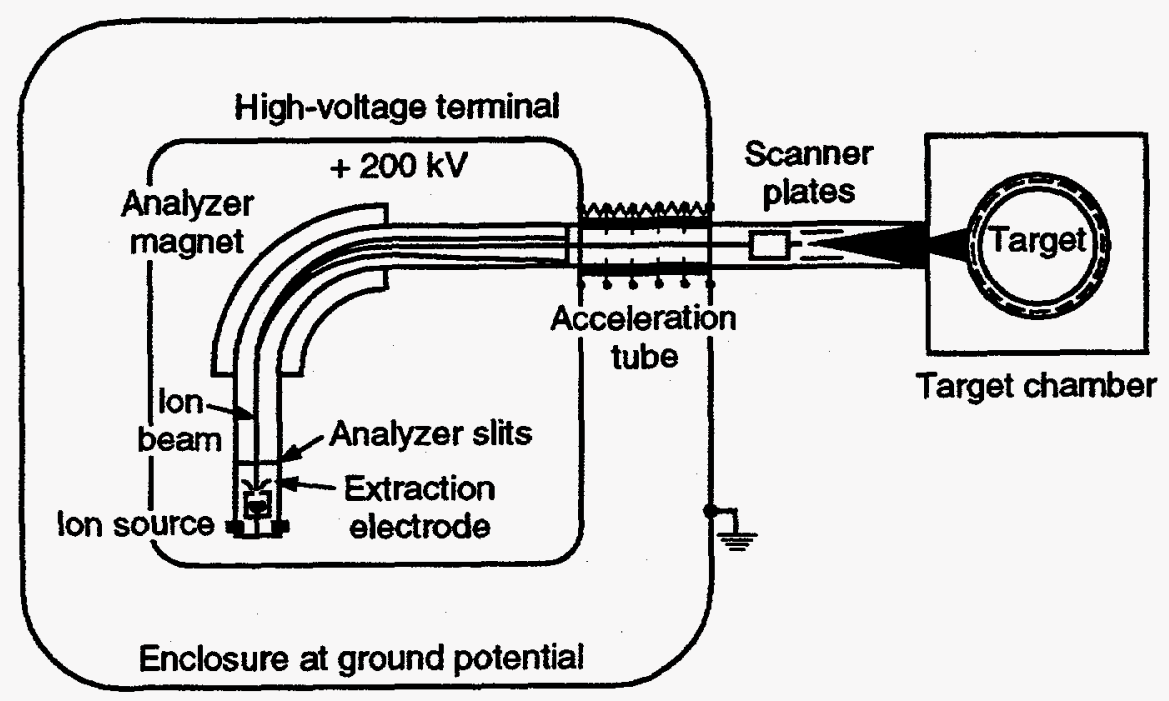

Figure 3.1d. Schematic diagram of beam-line ion implanter. 
implanted. Because the range of molecular ions is different from atomic ions, the resultant implant profile consists of ions of different energies.

Average current densities that can be achieved with PSII are two to three orders of magnitude higher than the beam-line implantation process. This reduces the time of implant at higher doses. In PSII, high beam currents are spread over the large surface, eliminating local beam heating problems. PSII does have limitations: precise determination of dose and energy is not easily possible, and plasma nonuniformities may lead to uneven implantation. There has been extensive work employing PSII to metallurgical applications $[31-39,46,123]$. Although, PSII has been traditionally developed as an implantation process, the same setup can be used for energetic ion depostion at low energies and high pressure.

Typically, if the biased voltage is less than $2 \mathrm{kV}$, the ions do not penetrate deep into the target (Eqn.3.1). Thus, at low voltage, ion beam deposition is favored. The energy of the deposition and the pressure in the chamber alter the deposition rates and energy distribution. It is well known from ion deposition experiments that the energy of the ion and the pressure are inversely related. If the pressure in the chamber is low, there are small numbers of collisions between the ions and gas atoms before being deposited on the substrate. Thus, the ions that are deposited on the substarte have high energy. On other hand, when the pressure in the chamber is high, ions undergo a large number of collisions with the gas atoms before being deposited on the substrate and lose energy. Thus, the ions that are deposited on the substrate have lower energy. Some important parameters in PSII processing are the gas pressure in the chamber, the amplitude of the biased voltage, and the rate at which the substrate is biased.

The ability to use a PSII setup either for ion implantation or for deposition is beneficial. For example, implantation followed by deposition in the synthesis of a coating produces an adherent interface. 


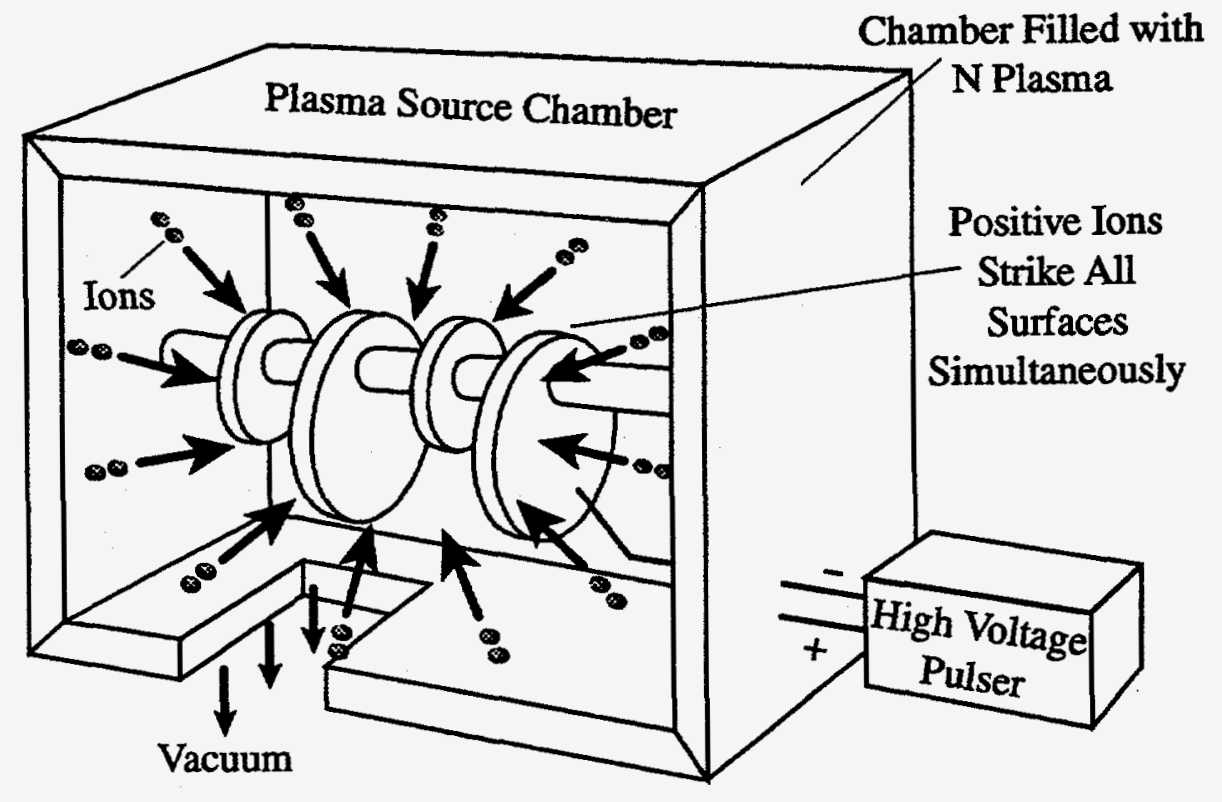

Figure 3.2. Schematic of the plasma source ion implantation from ref.[16]. 


\subsubsection{Magnetron Sputtering}

Plasma-source ion implantation is currently limited to gaseous ions such $\mathrm{N}$, hydrocarbons, Ar, etc. In some tribological applications, ceramic or metallic coatings are considered as potential candidates to improve the performance of the workpiece. Sputtering is one processing method used routinely to synthesize ceramic and metallic coatings. Sputtering systems can be easily automated and are widely used. The quality and composition of the films produced by sputtering are highly reproducible. In addition, successfully developed novel coatings by sputtering can be considered as potential candidates for the PSII process.

Sputtering is the ion-based material deposition by which the process of momentum transfer from an energetic ion to a solid target results in the ejection of atoms or molecules from the target surface. In sputter deposition, the sputtered atoms are collected on the substrate to form a film or a coating. A typical sputtering deposition setup is shown in the Figure 3.3. The target is a material from which the coating is synthesized and is connected to the negative terminal of a DC power supply. The substrate onto which the coating is deposited faces the target. The substrate may be grounded, biased positively or negatively, heated, cooled, or some combination of these conditions. The field generated by a magnet located behind the target helps trap the electrons, thus increasing the efficiency of ionization. After the chamber has been evacuated, a working gas, typically argon, is introduced into the chamber. This gas serves as the medium in which the discharge is initiated or sustained. Since the target is negatively biased, the positive ions from the plasma strike the target and eject the target atoms through momentum transfer. Some of these atoms eventually deposit on the substrate. The energy of neutral atoms that are deposited on the substrate and the sputtering gas pressure follow an inverse relationship. Important parameters in the 


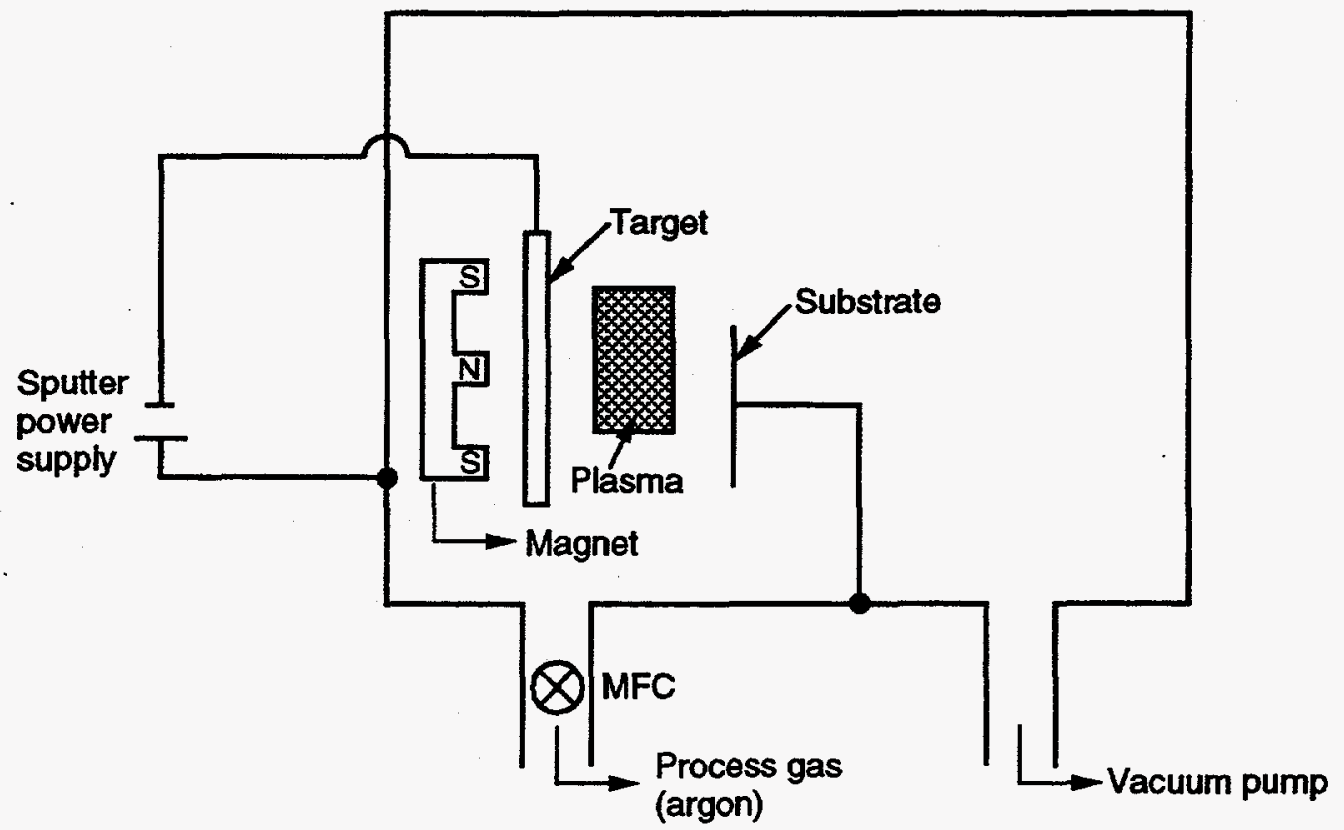

Figure 3.3. Schematic diagram of DC magnetron sputtering. 
deposition process are the distance between target and the substrate, substrate temperature, sputter gas pressure, and the sputter voltage.

\subsection{Characterization Techniques}

The performance of the coatings are strongly influenced by their properties, such as thickness, density, chemical composition, microstructure, etc. These coating properties depend on the process method and parameters employed. Thus, in the scientific development of new coatings, synthesis should be followed by characterization to evaluate coating properties. Once the properties are identified, the performance and potential applications of these coatings can be evaluated.

Additionally, in industrial applications, if the coatings are developed for a specific application, characterization of these coatings is vital for quality control. Thus, detailed characterization of a coating is important both in scientific development and for industrial applications.

Figure 3.4 shows the flow chart of steps that are necessary in the development of coatings for tribological applications. Coating thickness, residual intrinsic stresses introduced from processing, microstructure, and the composition are related to the processing parameters and determine the properties of the coating. A crucial step in evaluating a tribological coating is the analysis of the wear track for wear mechanisms, transfer of materials, tribochemical reactions, and the coating-substrate integrity. Thus, in order to evaluate the tribological performance of a coating at given test parameters, it is necessary to investigate microstructure, composition, and mechanical properties. The rest of this chapter describes the experimental techniques that were used to characterize the coatings.

\subsubsection{Microstructural Investigations}

Transmission electron microscopy (TEM) was used to examine the changes in microstructure after surface modification. Cross-sectional samples were prepared by 


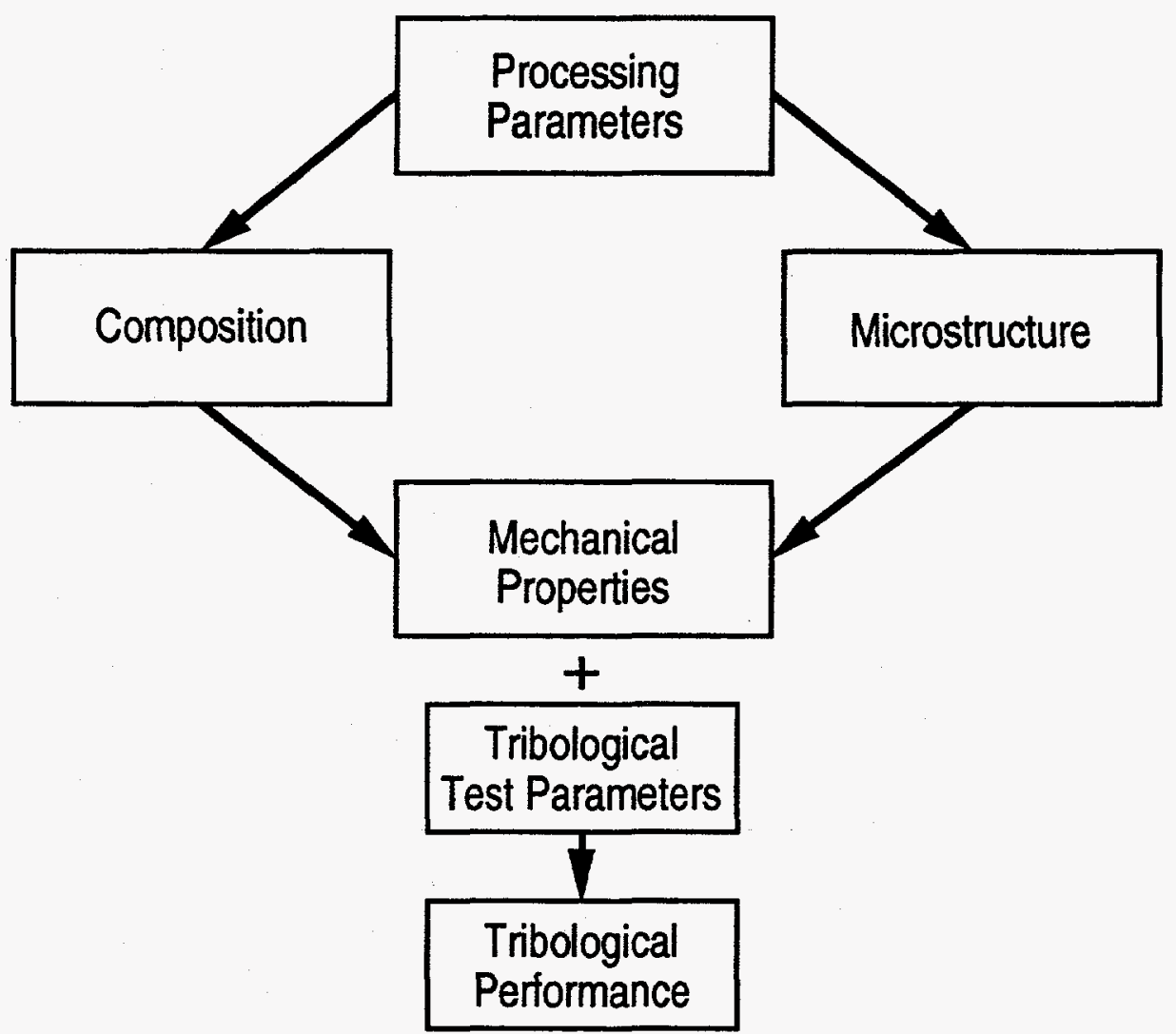

Figure 3.4. Flow chart to evaluate the tribological performance of a coating. 
standard techniques and examined using both Jeol-2000FX and Phillips CM30 microscopes.

\subsubsection{Composition Analysis}

Ion-beam analysis was used for compositional analysis. Rutherford Backscattering Spectrometry (RBS) was used to determine the retained dose, changes in composition, and location of the peak dopant concentration after implantation. Crystallinity and depth of the damaged layer were examined by ion channeling. A $2 \mathrm{MeV} \mathrm{He}$ ion beam was used for both channeling and RBS experiments. Details of the channeling measurements and experiments are given in a recent handbook on ion beam analysis [124]. Backscattered spectra were analyzed using the RUMP code [125]. A schematic diagram of the backscattering spectrometry accelerator used for the ion-beam analysis is shown in Figure 3.5.

Forward recoil elastic spectrometry was used to detect the hydrogen in DLC coatings. In this technique, instead of measuring the energy of backscattering helium ions, the energies of emitted hydrogen ions are measured. The details of this technique have been discussed by J.C. Barbour and B. L. Doyle in Ref. [124]. A 2 $\mathrm{MeV} \mathrm{He} e^{+}$ion beam was used for this analysis.

\subsubsection{Residual Stress Measurements}

Intrinsic stresses caused by processing were calculated by measuring the radius of curvature of silicon samples before and after deposition. The stress in thin films deposited on a single crystal can be determined using the Stoney equation [126],

$$
\sigma=\frac{E_{s} t_{s}^{2}}{6 t_{f}}\left[\Gamma_{d}-\Gamma_{s}\right]
$$

where:

$\sigma$ is the stress, 


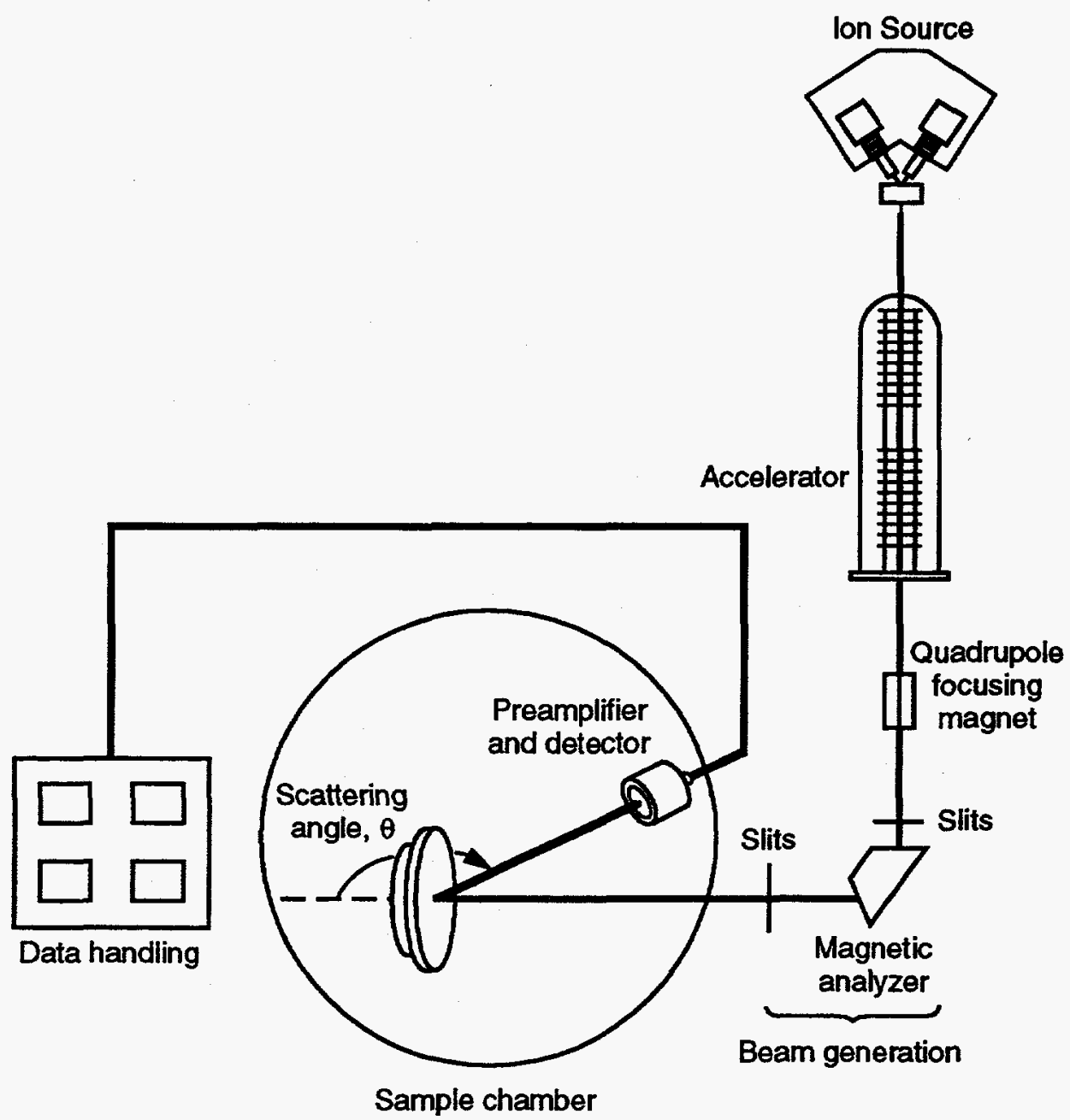

Figure 3.5. Schematic diagram of a backscattering spectrometry accelerator. 
$\mathrm{E}_{\mathrm{s}}$ is the bi-axial modulus of the substrate,

$t_{s}$ and $t_{f}$ are the thickness of the substrate and film, and

$\Gamma_{\mathrm{d}}$ and $\Gamma_{\mathrm{s}}$ are the curvatures of the deposited and uncoated silicon, respectively.

For measuring stresses, specimens of size $5 \times 38 \mathrm{~mm}$ in dimension were cut from single crystal $\mathrm{Si}(100)$ wafers.

\subsubsection{Hardness Measurements}

Hardness measurements were performed using a nanoindenter. Because the depth of the microindent is always larger than the thickness of the modified layer (0.1$5 \mu \mathrm{m})$, conventional hardness testers cannot be used to measure the hardness properties of thin surface modified layers. Moreover, in conventional microhardness tests, the hardness is obtained by imaging the indent after the the load is removed. When the indents are small as those in a nanoindenter, large errors are introduced when the indent diagonal lengths are measured. To measure the hardness of the thin modified layers, indents have to be very small. Depth sensing instruments must be employed to measure hardness of thin modified layers [127]. The depth measured during the indentation includes both plastic and elastic displacements. To obtain the hardness, the elastic contribution must be subtracted from the data.

A schematic diagram of the nanoindenter setup is shown in Figure 3.6a.

A Berkovich indenter is a three-sided pyramid and was used as the indenter tip. A capacitance gauge was used to determine the position of the indenter. A coil and a magnet assembly located at the top of the loading column were used to drive the indenter towards the sample. The force imposed on the column was controlled by varying the current in the coil. The loading column was suspended by flexible springs, and the motion was damped by air flow around the center plate of the capacitor, which was attached to the loading column. 
A typical nanoindentation test involved moving the indenter towards the surface of the material and measuring the forces and displacements associated with the indentation process. The surface was located for each indentation by lowering the indenter at a constant rate against suspending springs and by detecting changes in the force on contact with the surface. In testing mode, the load was incremented to maintain a constant velocity. The load and indentation depths were measured during both the loading and unloading cycles. The force contribution of the suspending springs and the displacements associated with the measured compliance of the instrument were removed.

A typical loading and unloading curve in a nanoindentation test is shown in the Figure 3.6b. The slope of the unloading curve is used to calculate the modulus of elasticity. The slope, $\mathrm{S}$, is given by

$$
S=\frac{1}{2 h_{p}}\left(\frac{\pi}{24.5}\right)^{1 / 2} \frac{1}{E_{r}}
$$

where:

$$
\frac{1}{E_{\mathrm{r}}}=\frac{1-v_{0}^{2}}{\mathrm{E}_{0}}+\frac{1-v_{1}^{2}}{\mathrm{E}_{1}},
$$

$h_{p}$ is the plastic depth,

$\mathrm{E}_{0}$ is the indenter modulus,

$v_{0}$ is the Poisson's ratio of indenter,

$\mathrm{E}_{1}$ is the sample modulus, and

$v_{1}$ is the Poisson's ratio of sample.

An assumption must be made on the value of Poisson's ratio of sample. The plastic depth can be calculated from the load-displacement curve by fitting a tangent to the unloading curve at maximum load and then extrapolating to zero load. 


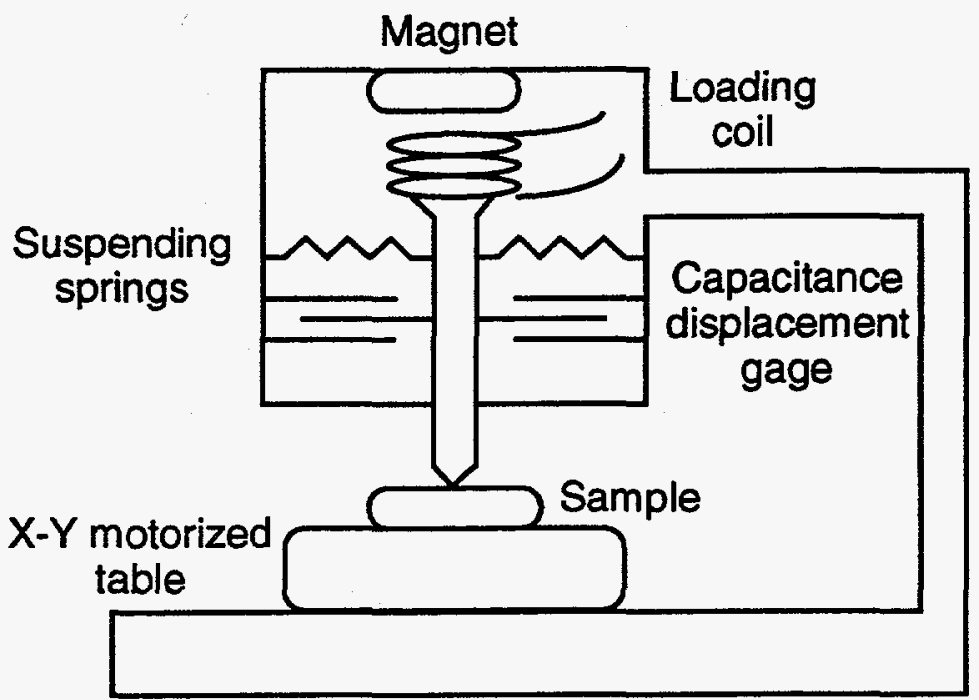

Figure 3.6a. Schematic diagram of the nanoindenter setup, from ref. [129].

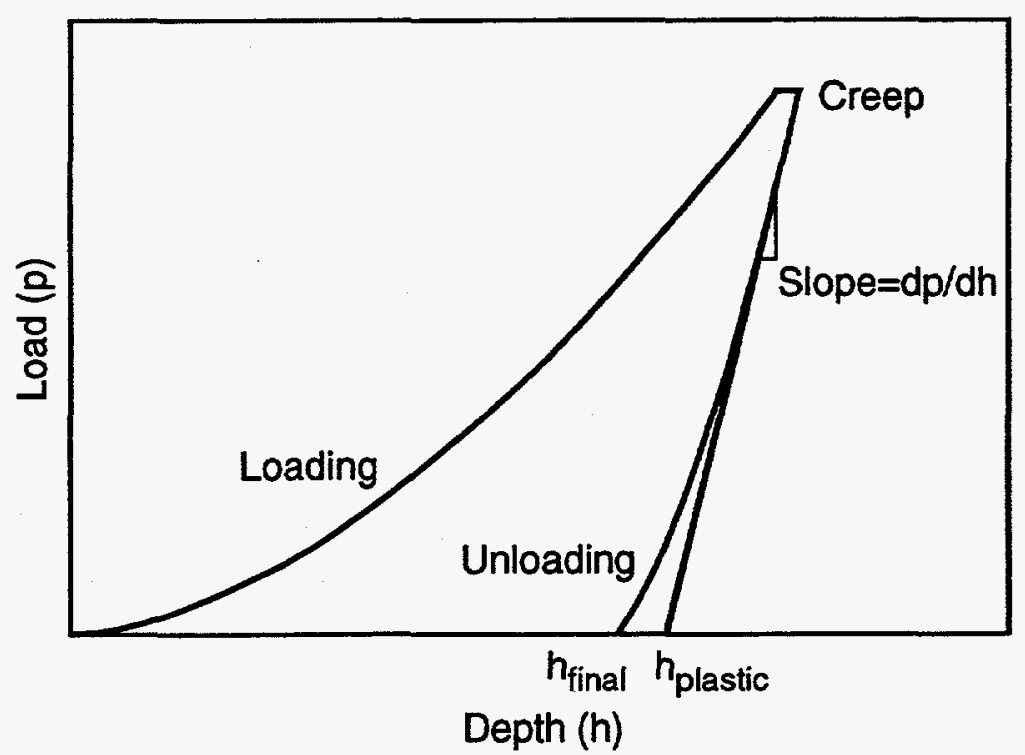

Figure 3.6b. Typical loading and unloading curve of an indentation test. 
The $\mathrm{x}$ intercept at the extrapolated zero crossing is known as the plastic depth. Since the plastic depth and the geometry of the indenter are known, the projected area in contact with the indenter can be calculated.

Hardness values were calculated using

$$
H=\frac{L(d)}{A(d)}
$$

where:

$\mathrm{L}(\mathrm{d})$ is the measured load, at a given depth

$A(d)$ is the projected area of the indent at a given depth, and

$\mathrm{d}$ is the displacement depth from the surface.

Multiple indents were made on each sample, and the data were averaged. By using plastic depth to obtain the projected area, the data were corrected for elasticity. The maximum indent depth was kept below $10 \%$ of the coating thickness to minimize substrate effects on the measured hardness and modulus $[128,129]$.

\subsubsection{Tribological Investigations}

Friction and wear properties of materials were performed using a pin-on-disc (POD) apparatus. Information on both friction and wear were obtained by a single test. The testing conditions are summarized in Table 3.2. Elastic moduli and Poisson's ratio of the uncoated silicon and the sliders are given in Table 3.3.

\subsubsection{Pin-on-Disc Apparatus}

Wear tests were performed without lubrication at room temperature in air at controlled humidity. Spheres of AISI 52100 steel and ruby $6 \mathrm{~mm}$ in diameter were used as the pin. In this setup, the pin was mounted on a weighted arm that rested on a flat rotating disc. The pin was examined for major processing defects using an optical microscope. This setup is shown in Figure 3.7. Before each wear test, both pin and disc were degreased by ultrasonic cleaning in acetone followed by isopropyl alcohol. 
The sliding speeds were changed by changing the rotation speed of the disc. The static contact stress for a sphere resting on a plane disc was calculated using Eqn. [2.6].

\subsubsection{Wear Measurements}

Wear area was determined by integrating the surface profiles of a wear track taken perpendicular to the sliding direction. In this study, wear area measurements were performed at four positions along the wear track. The ridges that were observed at the sides of the wear track that were caused by the plastic deformation of the discs were neglected. To compare the various samples, the wear factor was calculated. The wear factor is defined as the wear volume per unit load per unit sliding distance and expressed as $\mathrm{mm}^{3} / \mathrm{N} \cdot \mathrm{m}[55]$.

The wear factor, $\mathrm{W}$, is given by

$\mathrm{W}=\frac{\mathrm{V}}{\mathrm{LF}_{\mathrm{N}}}$

where:

$$
\begin{aligned}
& \mathrm{V}=\text { Wear volume }\left(\mathrm{mm}^{3}\right), \\
& \mathrm{L}=\text { Total sliding distance }(\mathrm{m}), \text { and } \\
& \mathrm{F}_{\mathrm{N}}=\text { Normal Load }(\mathrm{N})
\end{aligned}
$$

\subsubsection{Wear Morphology}

The morphology of the wear tracks was examined with a high-resolution field emission gun JEOL6300 scanning electron microscope and a Nanoscope-III AFM. The atomic force microscope (AFM) was operated in contact and tapping modes, with silicon nitride and silicon tips, respectively.

\subsubsection{Fracture Toughness Measurements}

Fracture toughness tests were performed using a Vickers indenter with loads ranging from 25 to 1000 grams. The indentation axis was normal to the specimen 


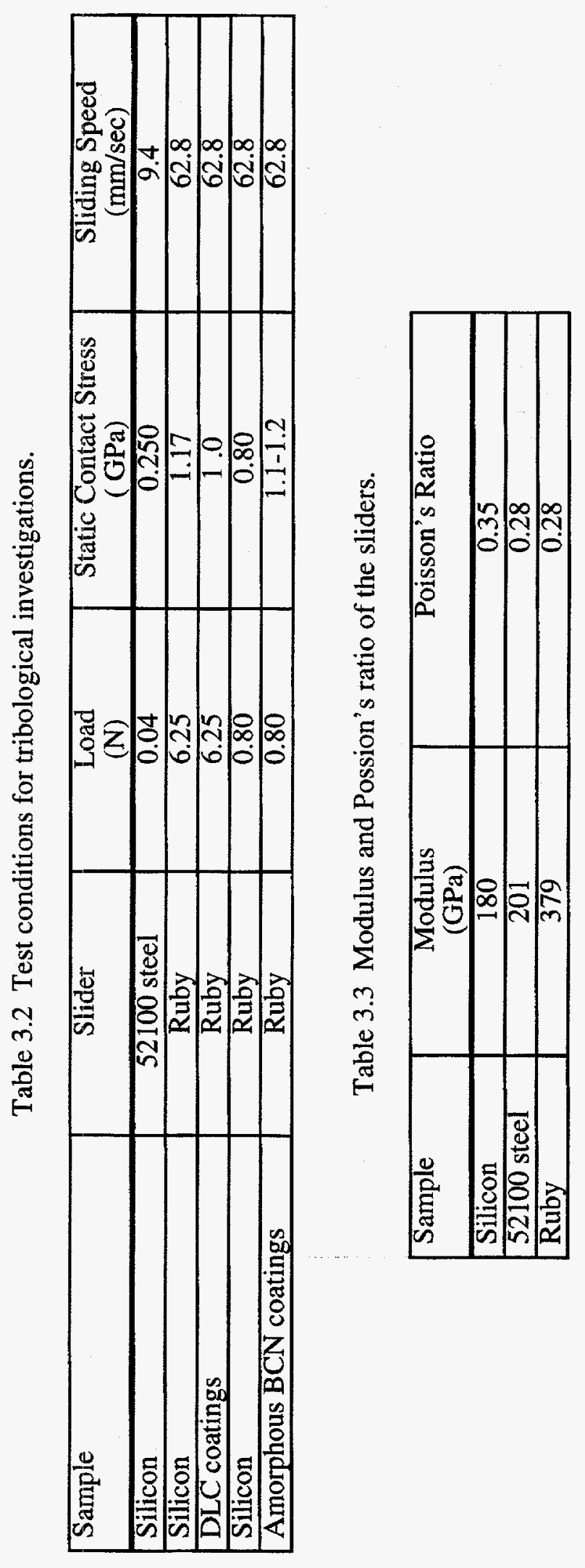




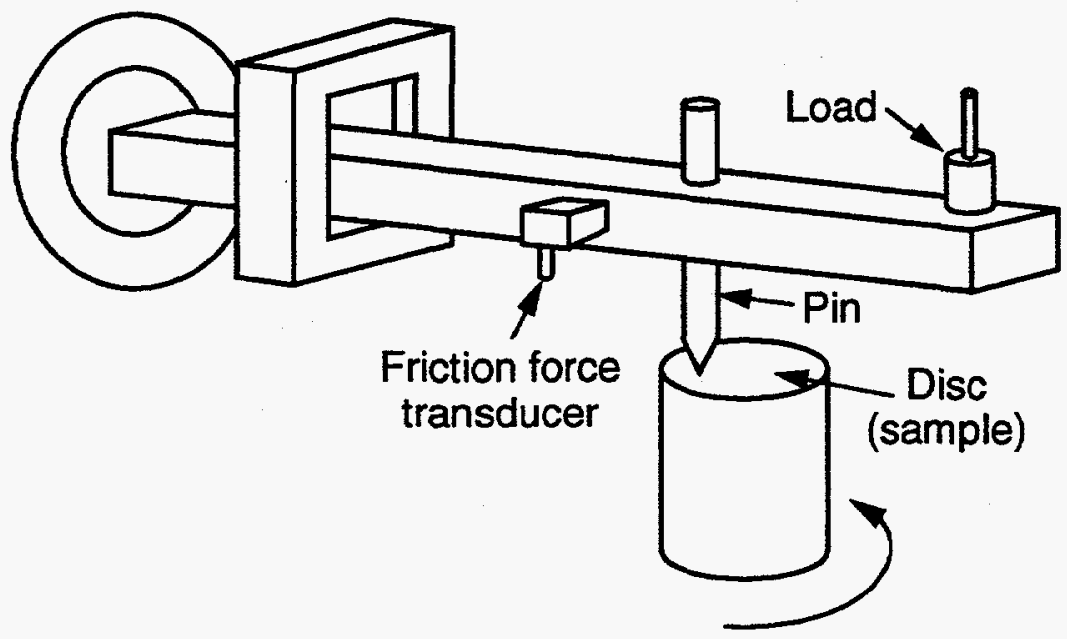

Figure 3.7. Schematic diagram of a POD apparatus to measure friction and wear. 
surface. The indentation cracks were parallel to the silicon (001) planes. The criteria for a well-defined crack is given by $C \geq 2 a$, where $C$ is the crack length and $2 a$ is the diagonal of the indent [130]. The crack length was determined by averaging the data of four indents made at every load. The residual stress intensity factor for this type of crack geometry is given by [130]

$$
\mathrm{K}_{\mathrm{r}}=\chi \frac{\mathrm{P}}{\mathrm{C}^{3 / 2}}
$$

where:

$P$ is the peak contact load, and

$\chi$ is a material constant. that depends on the hardness,

For brittle materials, $\chi$ is taken as $0.016(\mathrm{E} / \mathrm{H})^{1 / 2}[130]$.

where:

$\mathrm{H}$ is the the hardness, and

$\mathrm{E}$ is the Young's modulus.

The residual stress intensity factor was taken as the fracture toughness of the material when cracks are at equilibrium. Figure 3.8 shows an indentation technique and the crack geometry used for these measurements.

The indentation technique can be used to determine the fracture toughness of coatings deposited on brittle substrates. In this case, the residual stress intensity factor comes from the superposition of stress intensity factors due to the film and the substrate. Thus, the fracture toughness of coating-substrate systems determined by this technique is referred to as the apparent or effective fracture toughness of the coating-substrate. 


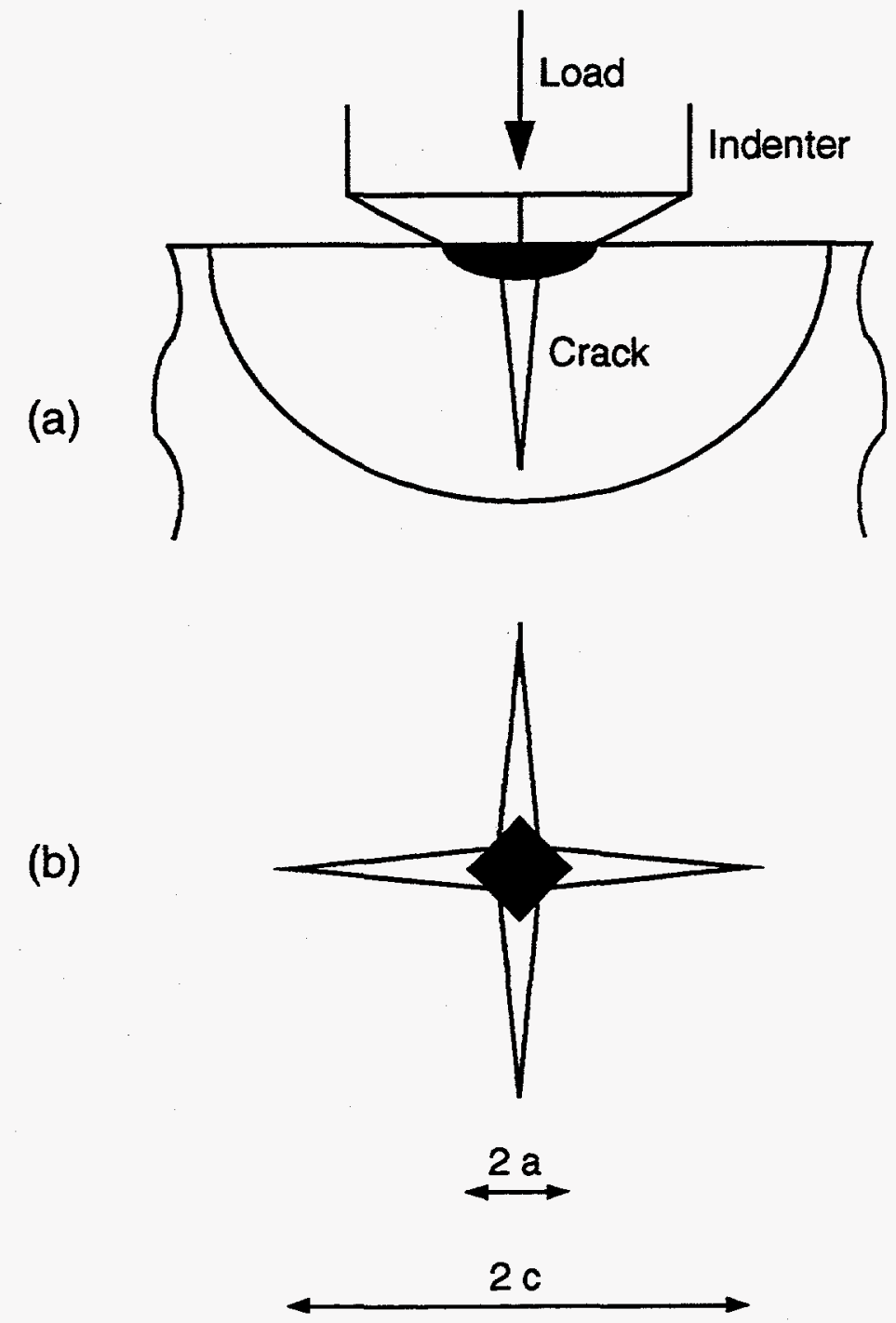

Figure 3.8. (a) Deformation and fracture due to Vickers indentation (side view). (b) Indentation pattern (plane view). 


\section{Chapter 4 Implantation Studies of Silicon}

In semiconductor technology, silicon is the dominant material used in microelectronic and micromechanical devices because of its excellent processing properties. The friction and wear behavior of implanted silicon has been addressed in several reports [22-26]. The research in these reports was aimed at understanding and controlling both friction and wear at microscopic and macroscopic levels by implantation of a single gas species into silicon [22-26]. However, there are no reports on the influence of implantation with multiple ion species into a single crystal silicon substrate. The aim in this study was to characterize the tribological and mechanical properties of a silicon substrates implanted with both carbon and nitrogen ions and to compare it with single implants of carbon and nitrogen. These mixed implantation studies on silicon crystal substrates are divided into two chapters. This chapter focuses on the characterization of implanted surfaces of silicon. Chapter 5 addresses the investigation of mechanical and tribological properties of implanted silicon.

\subsection{Objective}

One objective of this study was to examine the predictive capability of ionsolid interaction theory with experimental results. Specifically, implantation parameters such as projected range, straggling, and retained dose were examined. Complete analysis required the investigation of the microstructure of the implanted layer. Thus, this chapter focuses on the calculation of implantation parameters using analytical expressions, computer simulations, and ion beam analysis.

\subsection{Implantation of Silicon}

Samples of single-crystal silicon with an orientation of (100) were implanted with ions of carbon and nitrogen with an energy of $60 \mathrm{keV}$ at room temperature using 
a beamline $200 \mathrm{keV}$ implanter. The sample was tilted 6-7 $7^{\circ}$ to minimize ion channeling effects. Typical ion implantation doses used were:

$$
\begin{aligned}
& 4 \times 10^{17} \mathrm{C}^{+} \text {ions } / \mathrm{cm}^{2}, \\
& 4 \times 10^{17} \mathrm{~N}^{+} \text {ions } / \mathrm{cm}^{2}, \\
& 4 \times 10^{17} \mathrm{C}^{+} \text {ions } / \mathrm{cm}^{2}+4 \times 10^{17} \mathrm{~N}^{+} \text {ions } / \mathrm{cm}^{2} \\
& 8 \times 10^{17} \mathrm{~N}^{+} \text {ions } / \mathrm{cm}^{2}, \text { and } \\
& 8 \times 10^{17} \mathrm{C}^{+} \text {ions } / \mathrm{cm}^{2} .
\end{aligned}
$$

To minimize surface contamination during implantation, a cryo shield surrounding the sample was cooled to liquid nitrogen temperatures. A background pressure of about $2 \times 10^{-6}$ Torr was maintained.

\subsection{Results}

The results of carbon and nitrogen implanted silicon are presented in the following order.

1) Determination of the projected range and straggling of the implanted species

2) Investigations of the microstructure

3) Characterization of the surface roughness

4) Discussion of the results

\subsubsection{Determination of Projected Ranges and Straggling of the Implanted Species}

The projected range and straggling of the implanted species were determined on the calculations using analytical expressions, computer simulations, and Rutherford backscattering spectrometry (RBS).

\subsubsection{Calculations from Analytical Expressions}

Analytical equations 3.1 to 3.8 described in Chapter 3 were used to estimate the projected range, $R_{p}$; straggling, $\Delta R_{p}$; peak atomic density, $N_{p}$; and peak atomic concentration, $\mathrm{C}_{\mathrm{p}}$; of a implanted species. The values of $\varepsilon$, the reduced energy, and $\mathrm{k}$, 
the stopping factor, for nitrogen-implanted silicon at $60 \mathrm{keV}$ were 4.3 and 0.25 , respectively. From Table 3.1, the reduced range, $\rho_{L}$, was 8.83 . The range of ions, $R$, from Eqn.[3.8] was determined as $297.4 \mathrm{~nm}$. The projected range, $R_{p}$, and straggling, $\Delta \mathrm{R}_{\mathrm{p}}$, for nitrogen-implanted silicon from Figure 3.1c were calculated to be $174.2 \mathrm{~nm}$ and $111.5 \mathrm{~nm}$, respectively. A similar analysis for $60 \mathrm{keV}$ carbon in silicon gave $183.6 \mathrm{~nm}$ and $116.1 \mathrm{~nm}$ for $R_{p}$, and $\Delta R_{p}$, respectively. These results are summarized in Table 4.1.

The mass and atomic number of carbon ions are lower than nitrogen ions, hence the projected range of carbon ions into silicon was higher than the nitrogen ions. The atomic peak concentration was calculated using Eqns. 3.2 and 3.3. The atomic peak concentration increased with dose. For example, the peak atomic fraction of nitrogen increased from 0.21 to 0.36 when the dose was increased from $4 \times 10^{17} \mathrm{~N}^{+}$ions $/ \mathrm{cm}^{2}$ to $8 \times 10^{17} \mathrm{~N}^{+}$ions $/ \mathrm{cm}^{2}$.

The analytical expressions used in the above analysis were derived for a constant homogenous composition target. These expressions cannot be used simply and accurately to determine the projected range, $R_{p}$, and straggling $\Delta R_{p}$, for evolving compositional changes or targets with non-homogenous composition. Thus, for mixed-implant specimens the projected range, $R_{p}$, and straggling, $\Delta R_{p}$, cannot be estimated from analytical expressions.

\subsubsection{Computer Simulations}

As discussed in Chapter 3, TRIM [105] is the most commonly used program to calculate ion ranges and damage. Figure 4.1 shows the profile of Monte Carlo simulations of carbon and nitrogen implantation into silicon. The results obtained from these simulations are summarized in Table 4.2. These data indicated that the carbon ions penetrated deeper than nitrogen ions, in agreement with analytical results, 
Table 4.1. The results for projected range were in reasonable agreement with estimated values from analytical expressions. However, the TRIM straggling was less than analytically estimated by a factor of 2 . This suggests that the analytical expressions that were used are not accurate.

For a mixed-implant sample, where both carbon and nitrogen were sequentially implanted into same sample, the simulations were done to estimate the projected range of nitrogen ions by assuming the target is composed of three layers. The first layer was assumed to be $100 \mathrm{~nm}$ thick and $100 \%$ silicon; the second layer was $300 \mathrm{~nm}$ thick and had 0.64 and 0.37 atomic fraction of silicon and carbon, respectively. The third layer was $1300 \mathrm{~nm}$ thick silicon. These simulations predicted the projected range and straggling of nitrogen ions in carbon implanted silicon as $134 \mathrm{~nm}$ and $36 \mathrm{~nm}$, respectively.

\subsubsection{Ion Beam Analysis}

Experimentally, the projected range, straggling, and atomic concentration of the implanted species were determined by RBS. The width of the modified layer was determined by channeling measurements.

\subsubsection{Rutherford Backscattering Spectrometry Analysis}

Figure 4.2a-b shows the RBS spectra of the carbon implanted samples to a doses of $4 \times 10^{17} \mathrm{C}^{+}$ions $/ \mathrm{cm}^{2}$ and $8 \times 10^{17} \mathrm{C}^{+}$ions $/ \mathrm{cm}^{2}$. The simulated spectra are also shown in Figures 4.2a-b. The data were fit to a Gaussian distribution from which the peak concentration, location of the peak concentration, and concentration full-width half maximum were calculated. These results are summarized in the Tables 4.3 and 4.4 .

The ion beam analysis was performed using RUMP [125]. To get a compostional analysis, the data was fit to a Gaussian form defined by the following expression. 


$$
f(x)=C_{p}\left[\exp \left(-4 \ln (2)\left[\frac{x-p(2)}{p(3)}\right]^{2}\right)\right]
$$

where:

$$
C_{p}=2 \sqrt{\frac{\ln (2)}{\pi}} \frac{p(1)}{p(3)}
$$

$\mathrm{p}(1)$ is the integral of the Gaussian (atoms $/ \mathrm{cm}^{2}$ ),

$\mathrm{p}(2)$ is the depth of the layer (atoms $/ \mathrm{cm}^{2}$ ),

$\mathrm{p}(3)$ is the full width half maximum (FWHM) of the Gaussian distribution (atoms $/ \mathrm{cm}^{2}$ ), and

$\mathrm{C}_{\mathrm{p}}$ is the mole fraction of implanted ion species.

Depth of the layer and FWHM could not be expressed in units of thickness since the information on the variation of density with depth was not available.

However, assuming the density of the modified layer was in the range of the crystalline silicon $5 \times 10^{22}$ atoms $/ \mathrm{cm}^{3}$, the projected range, $R_{p}$, and the straggling, $\Delta R_{p}$, of the ions could be calculated. These data are summarized in Table 4.4. 


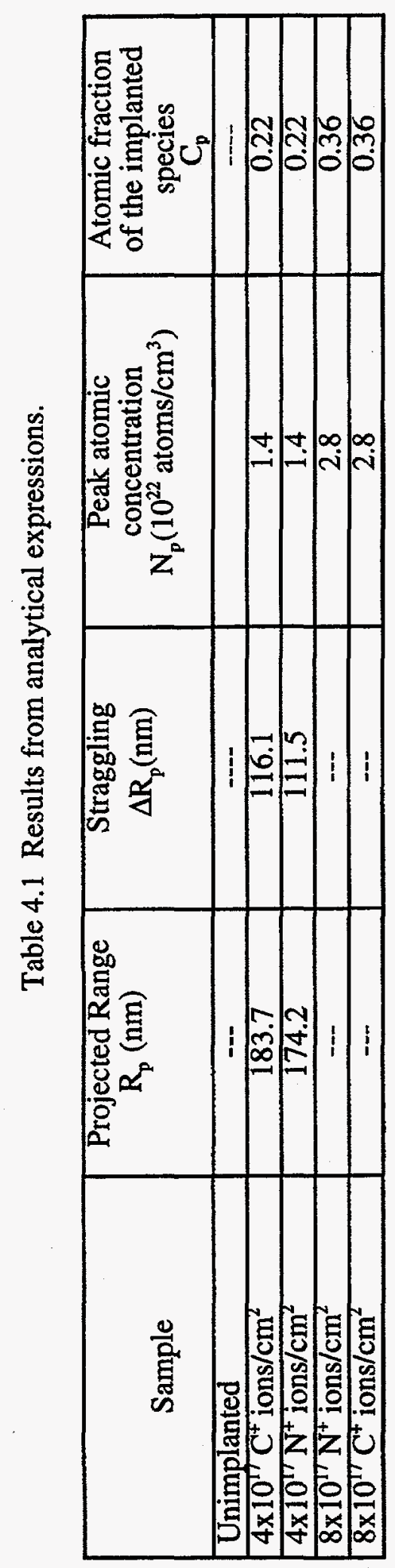




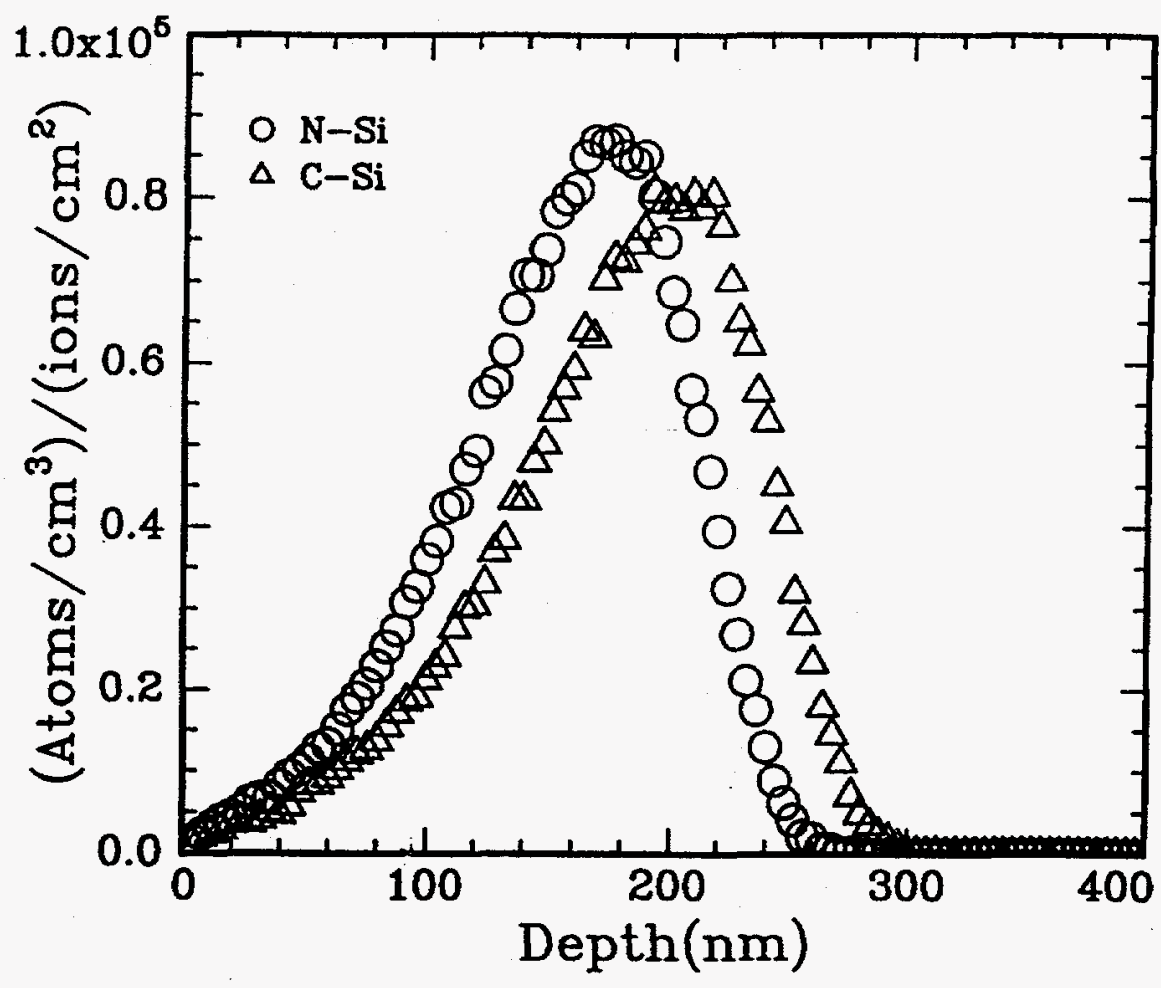

Figure 4.1 Implanted profile of carbon and nitrogen ions from Monte Carlo simulations. 
Table 4.2 Projected range and straggling of carbon and nitrogen ions into $\mathrm{Si}(100)$ from Monte Carlo simulations using TRIM [105].

\begin{tabular}{|c|c|c|c|c|}
\hline Sample & $\begin{array}{l}\text { Projected Range } \\
\mathrm{R}_{\mathrm{p}}(\mathrm{nm})\end{array}$ & $\begin{array}{r}\text { Straggling } \\
\Delta \mathrm{R}_{\mathrm{p}}(\mathrm{nm})\end{array}$ & $\begin{array}{c}\text { Peak atomic } \\
\text { Concentration } \\
N_{p} \\
\left(10^{22} \text { atoms } / \mathrm{cm}^{3}\right) \\
\end{array}$ & $\begin{array}{c}\text { Atomic fraction } \\
\text { of the implanted } \\
\text { species } \\
\mathrm{C}_{\mathrm{p}}\end{array}$ \\
\hline Unimplanted & -- & -- & -- & $\cdots$ \\
\hline $4 \times 10^{1 /} \mathrm{C}^{+}$ions $/ \mathrm{cm}^{2}$ & 177.3 & 52.3 & 3.29 & 0.37 \\
\hline $4 \times 10^{1 /} \mathrm{N}^{+}$ions $/ \mathrm{cm}^{2}$ & 152.8 & 48.7 & 3.06 & 0.40 \\
\hline $8 \times 10^{1 /} \mathrm{N}^{+}$ions $/ \mathrm{cm}^{2}$ & 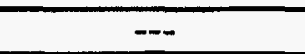 & $-\ldots$ & 6.12 & 0.57 \\
\hline $8 \times 10^{1 /} \mathrm{C}^{+}$ions $/ \mathrm{cm}^{2}$ & $\ldots$ & -- & 6.60 & 0.55 \\
\hline
\end{tabular}




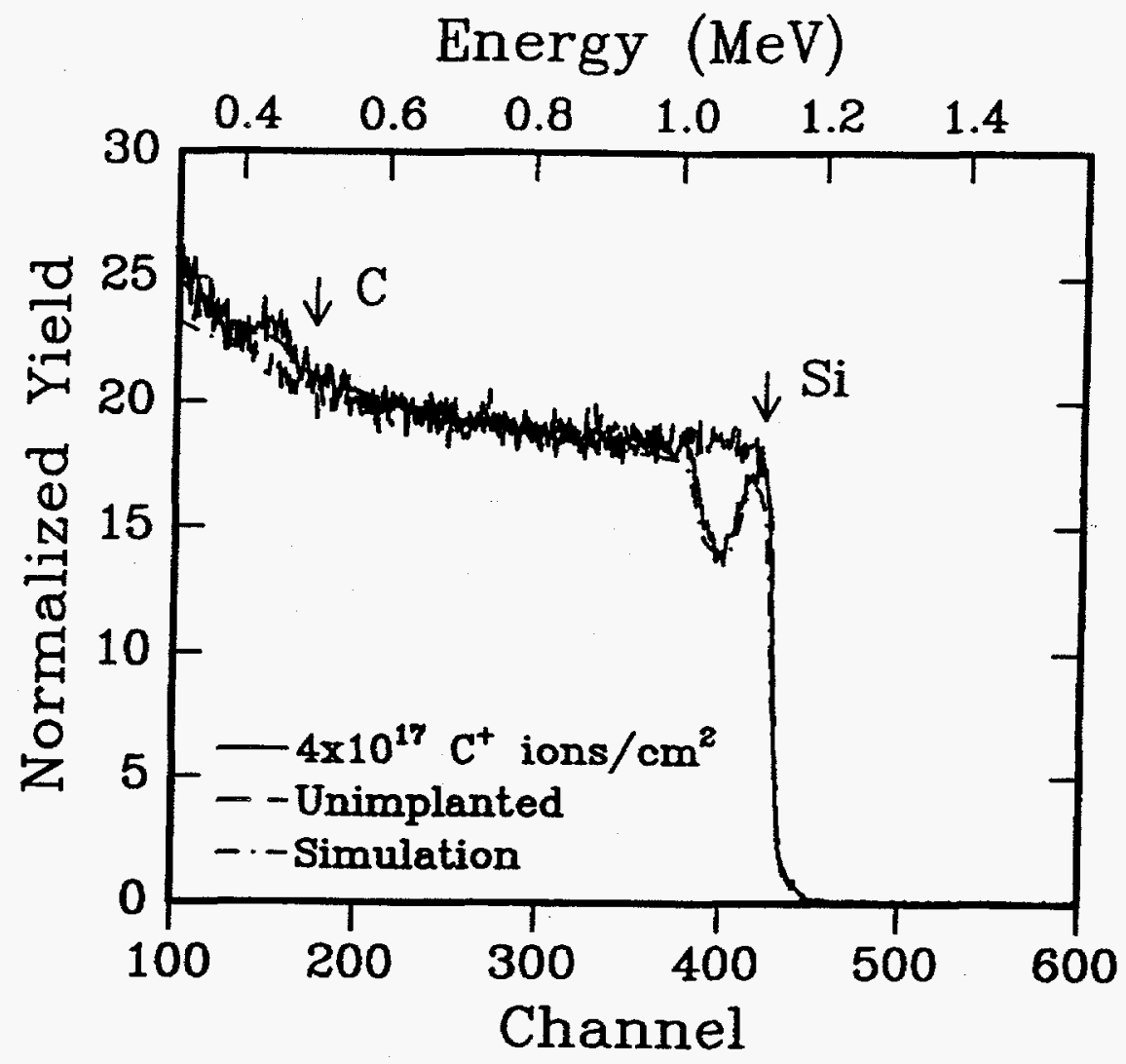

Figure 4.2a Rutherford backscattering spectra of the sample implanted with $4 \times 10^{17} \mathrm{C}^{+}$ions $/ \mathrm{cm}^{2}$. 


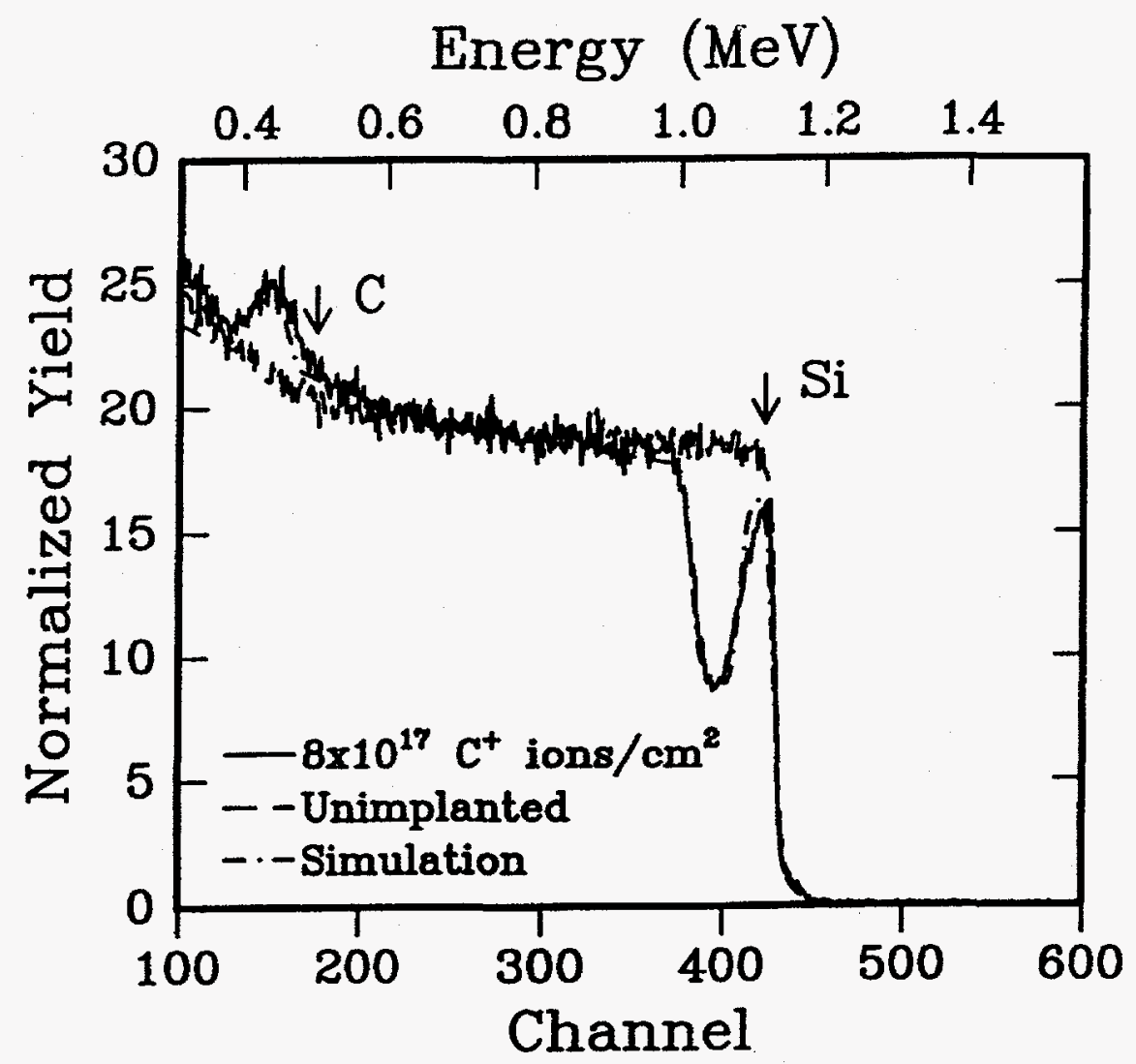

Figure 4.2b Rutherford backscattering spectra of the sample implanted with $8 \times 10^{17} \mathrm{C}^{+}$ions $/ \mathrm{cm}^{2}$. 
RBS studies indicated that the peak atomic fraction of the nitrogen ions was approximately 0.47 in the sample implanted with $4 \times 10^{17} \mathrm{~N}^{+}$ions $/ \mathrm{cm}^{2}$. When the dose was increased to $8 \times 10^{17} \mathrm{~N}^{+}$ions $/ \mathrm{cm}^{2}$, the concentration of nitrogen ions changed to 0.72 . This peak atomic fraction of nitrogen ions was greater than the amount of nitrogen (0.57) required to form stoichiometric silicon nitride $\left(\mathrm{Si}_{3} \mathrm{~N}_{4}\right)$. Similarly, the peak concentration increased with dose for the carbon-implanted samples. For the sample implanted with $8 \times 10^{17} \mathrm{C}^{+}$ions $/ \mathrm{cm}^{2}$, the peak concentration was greater than the amount of carbon (50\%) required to form stoichiometric silicon carbide (SiC).

In the mixed-implant sample, the measured range of nitrogen ions was $78 \mathrm{~nm}$ as compared to $160 \mathrm{~nm}$, the range in silicon. The carbon-implanted sample had a higher density of scattering centers as compared to unimplanted silicon. For example, $\mathrm{N}_{\mathrm{si}}=5 \times 10^{22}$ atoms $/ \mathrm{cm}^{3}$, whereas $\mathrm{N}_{\mathrm{SiC}}=7.4 \times 10^{22}$ atoms $/ \mathrm{cm}^{3}$. As a result, a higher energy loss $(\mathrm{dE} / \mathrm{dx})$ can occur in the carbon-implanted sample, leading to a reduction in the projected range of the ions relative to pure silicon. Thus, the range of nitrogen ions in carbon-implanted silicon was less than in silicon. 
Table 4.3 Results from RBS analysis in units of $10^{17}$ atoms $/ \mathrm{cm}^{2}$.

\begin{tabular}{|c|c|c|c|c|}
\hline Sample & $\begin{array}{c}\mathrm{p}(1) \\
10^{17} \text { atoms } / \mathrm{cm}^{2}\end{array}$ & $\begin{array}{c}\mathrm{p}(2) \\
10^{17} \text { atoms } / \mathrm{cm}^{2}\end{array}$ & $\begin{array}{c}\mathrm{p}(3) \\
10^{17} \text { atoms } / \mathrm{cm}^{2}\end{array}$ & $\begin{array}{l}\text { Mole fraction of } \\
\text { the implanted } \\
\text { species } \\
\mathrm{C}_{\mathrm{p}}\end{array}$ \\
\hline Unimplanted & $\overline{-\cdots}$ & $\overline{---}$ & $-\cdots$ & $\cdots$ \\
\hline $4 \times 10^{1 /} \mathrm{C}^{+}$ions $/ \mathrm{cm}^{2}$ & 2.0 & 9.0 & 4.6 & 0.40 \\
\hline $4 \times 10^{1 /} \mathrm{N}^{+}$ions $/ \mathrm{cm}^{2}$ & 3.7 & 8.0 & 7.2 & 0.47 \\
\hline $8 \times 10^{1 /} \mathrm{N}^{+}$ions $/ \mathrm{cm}^{2}$ & 6.9 & 9.0 & 8.9 & 0.73 \\
\hline $8 \times 10^{11} \mathrm{C}^{+}$ions $/ \mathrm{cm}^{2}$ & 6.8 & 10.0 & 8.9 & 0.72 \\
\hline \multicolumn{5}{|c|}{$4 \times 10^{17} \mathrm{C}^{+}$ions $/ \mathrm{cm}^{2}+4 \times 10^{11} \mathrm{~N}^{+}$ions $/ \mathrm{cm}^{2}$} \\
\hline C-portion of the implant & 4.0 & 10.8 & 6.2 & 0.61 \\
\hline $\mathrm{N}$-portion of the implant & 4.0 & 3.9 & 5.6 & 0.67 \\
\hline
\end{tabular}


Table 4.4 Results from RBS analysis in units of $\mathrm{nm}$.

\begin{tabular}{|c|c|c|c|c|}
\hline Sample & $\begin{array}{l}\text { Retained Dose } \\
10^{17} \text { atoms } / \mathrm{cm}^{2}\end{array}$ & $\begin{array}{l}\mathrm{R}_{\mathrm{p}_{\mathrm{a}}} \\
\mathrm{nm}^{\mathrm{a}}\end{array}$ & $\begin{array}{c}\overline{\text { FWHM }} \\
\mathrm{nm}^{\mathrm{a}}\end{array}$ & $\begin{array}{l}\Delta \mathrm{R}_{\mathrm{p}} \\
\mathrm{nm}^{2}\end{array}$ \\
\hline Unimplanted & -- & $\overline{---}$ & -- & $\overline{-n-\infty}$ \\
\hline $4 \times 10^{1 /} \mathrm{C}^{+}$ions $/ \mathrm{cm}^{2}$ & 2.0 & 180 & $\overline{92}$ & 39 \\
\hline $4 \times 10^{1 /} \mathrm{N}^{+}$ions $/ \mathrm{cm}^{2}$ & 3.7 & 160 & 144 & 61.1 \\
\hline $8 \times 10^{17} \mathrm{~N}^{+}$ions $/ \mathrm{cm}^{2}$ & 6.9 & 180 & 178 & $\overline{75.6}$ \\
\hline $8 \times 10^{11} \mathrm{C}^{+}$ions $/ \mathrm{cm}^{2}$ & 6.8 & 200 & 178 & 75.6 \\
\hline \multicolumn{5}{|c|}{$4 \times 10^{1 /} \mathrm{C}^{+}$ions $/ \mathrm{cm}^{2}+4 \times 10^{1 /} \mathrm{N}^{+}$ions $/ \mathrm{cm}^{2}$} \\
\hline C-portion of the implant & 4.0 & 216 & $\overline{124}$ & $\overline{38.6}$ \\
\hline $\mathrm{N}$-portion of the implant & 4.0 & 78 & $\overline{112}$ & $\overline{47.6}$ \\
\hline
\end{tabular}

a : Assuming the density of the modified layer is $5.0 \times 10^{22}$ atoms $/ \mathrm{cm}^{3}$. 


\subsubsection{Channeling Measurements}

Crystal orientation influence on ion penetration is known as channeling. If a single crystal is oriented with a major pole parallel to the incident beam direction, the yield from backscattered ions is small since the channeled ions do not make close impact collisions with the lattice atoms. Channeled ions also have a lower rate of energy loss, and hence a greater range than those of nonchanneled ions. This technique can be used to calculate the crystallinity of the ion-implanted region [124].

Figures 4.3a-b show the channeling spectra of the unimplanted sample and the nitrogen-implanted samples, i.e., $\left(4 \times 10^{17} \mathrm{~N}^{+}\right.$ions $/ \mathrm{cm}^{2}$ and $8 \times 10^{17} \mathrm{~N}^{+}$ions $\left./ \mathrm{cm}^{2}\right)$. These spectra indicated that the yield was the same for the channeled and random directions, suggesting that the modified layer could be amorphous or polycrystalline, with grains oriented at random. TEM studies performed to confirm the crystallinity of this modified layer revealed the amorphous nature of the layer. These results are discussed in section 4.4. The channeling data of other implanted samples, which are not shown here, indicated that the crystallinity of implanted layers was similar to those shown in Figures 4.3a-b. The width of the modified ion layer, including implantation and radiation damage, was calculated from these channeling data using RUMP [125]. The width of the ion-modified layer was observed to increase with dose. The results are summarized in Table 4.5. 
Table 4.5 Width of the ion-modified layer from channeling measurements.

\begin{tabular}{|l|c|}
\hline \multicolumn{1}{|c|}{ Sample } & $\begin{array}{c}\text { Width of the } \\
\text { ion-modified layer } \\
\text { from channeling } \\
\text { measurements (nm) }\end{array}$ \\
\hline Unimplanted & ---- \\
\hline $4 \times 10^{11} \mathrm{C}^{+}$ions $/ \mathrm{cm}^{2}$ & 300 \\
\hline $4 \times 10^{11} \mathrm{~N}^{+}$ions $/ \mathrm{cm}^{2}$ & 290 \\
\hline $4 \times 10^{1 /} \mathrm{C}^{+}$ions $/ \mathrm{cm}^{2}+4 \times 10^{1 /} \mathrm{N}^{+}$ions $/ \mathrm{cm}^{2}$ & 350 \\
\hline $8 \times 10^{11} \mathrm{~N}^{+}$ions $/ \mathrm{cm}^{2}$ & 320 \\
\hline $8 \times 10^{17} \mathrm{C}^{+}$ions/cm & 330 \\
\hline a: Includes implantation and radiation damage. \\
\hline
\end{tabular}

\subsection{Microstructural Investigations}

To investigate the crystallinity of the ion-modified layers, TEM studies were performed. Figure 4.4 shows a typical selected area diffraction pattern of the modified layers. The diffraction patterns have diffused rings, indicating the amorphous nature of the modified layers at all doses. The bright-field images of the samples implanted with $4 \times 10^{17} \mathrm{~N}^{+}$ions $/ \mathrm{cm}^{2}, 8 \times 10^{17} \mathrm{~N}^{+}$ions $/ \mathrm{cm}^{2}, 8 \times 10^{17} \mathrm{C}^{+}$ions $/ \mathrm{cm}^{2}$, and the mixed implant $\left(4 \times 10^{17} \mathrm{C}^{+}\right.$ions $/ \mathrm{cm}^{2}+4 \times 10^{17} \mathrm{~N}^{+}$ions $\left./ \mathrm{cm}^{2}\right)$ are shown in Figures 4.5 to 4.8 .

Figure 4.5 shows the TEM bright-field image of the sample implanted with $4 \times 10^{17} \mathrm{~N}^{+}$ions $/ \mathrm{cm}^{2}$. This micrograph indicates the modified layer was amorphous. When the dose was increased to $8 \times 10^{17} \mathrm{~N}^{+}$ions $/ \mathrm{cm}^{2}$, TEM data revealed bubble formation in the entire modified region (Figure 4.6). In contrast, for the sample implanted with $8 \times 10^{17} \mathrm{C}^{+}$ions $/ \mathrm{cm}^{2}$, no bubbles were observed (Figure 4-7). At a high 


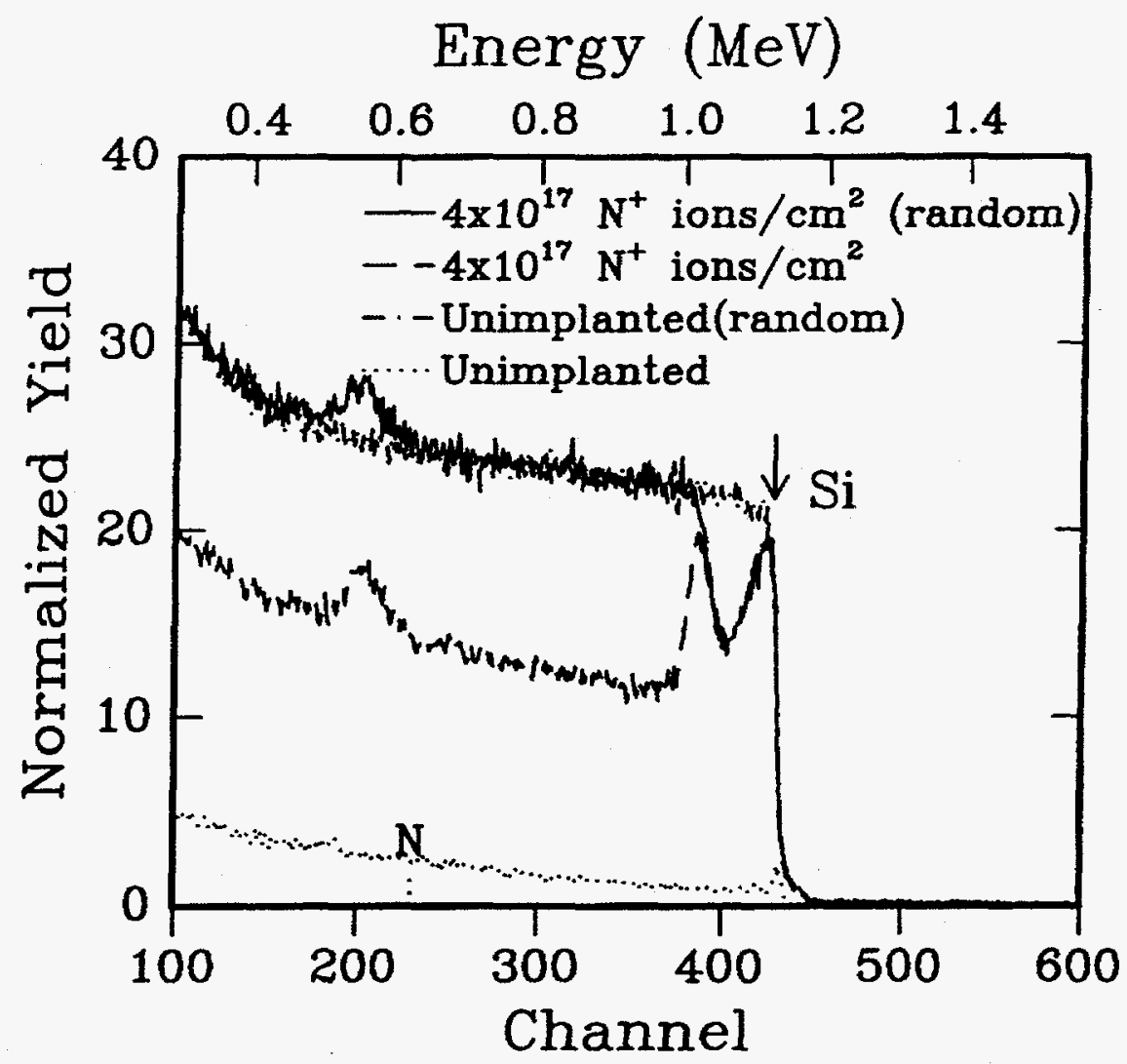

Figure 4.3a Channeling spectra of the sample implanted with $4 \times 10^{17} \mathrm{~N}^{+}$ions $/ \mathrm{cm}^{2}$ and the uncoated silicon. 


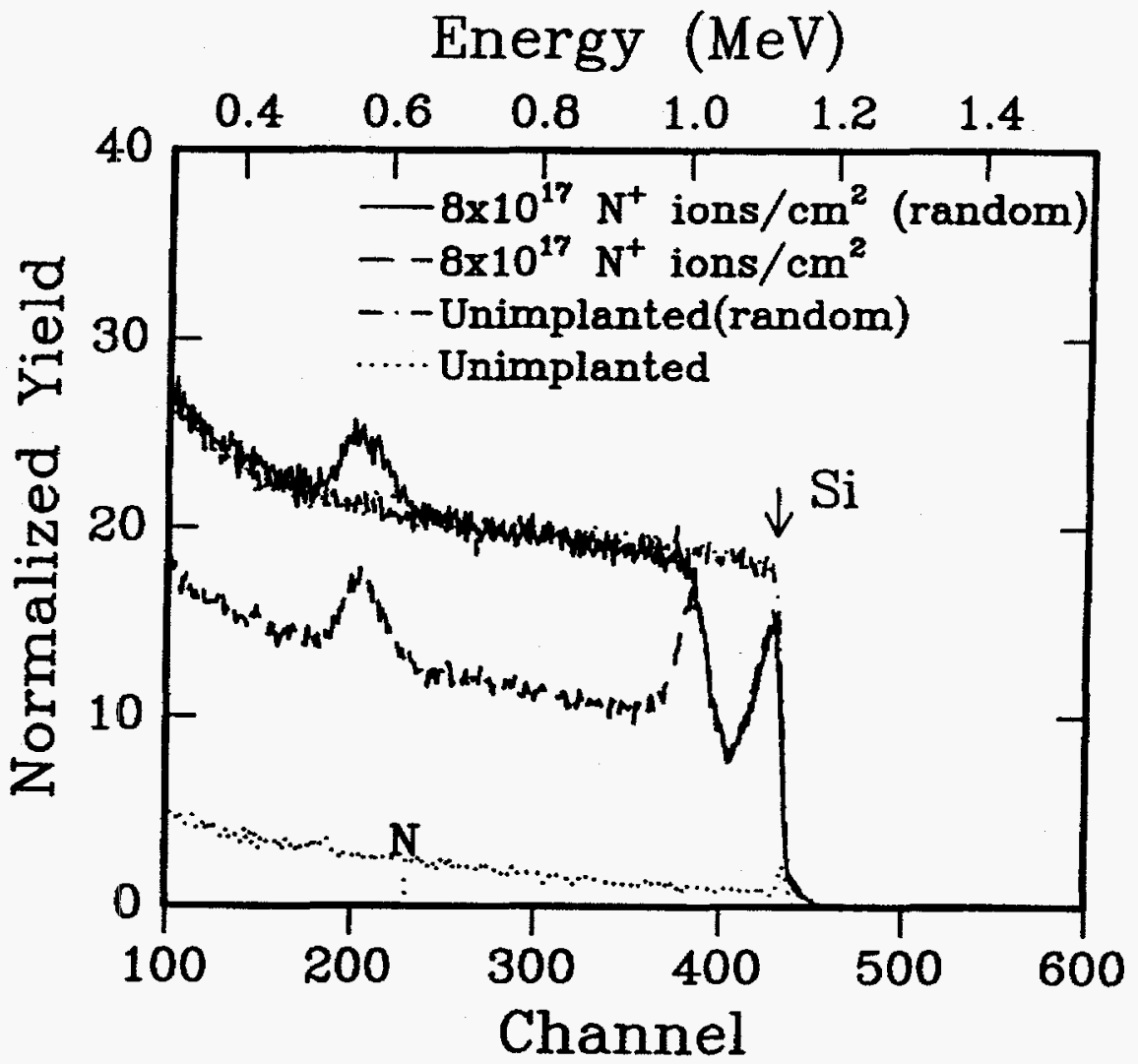

Figure 4.3b Channeling spectra of the sample implanted with $8 \times 10^{17} \mathrm{~N}^{+}$ions $/ \mathrm{cm}^{2}$ and the uncoated silicon. 


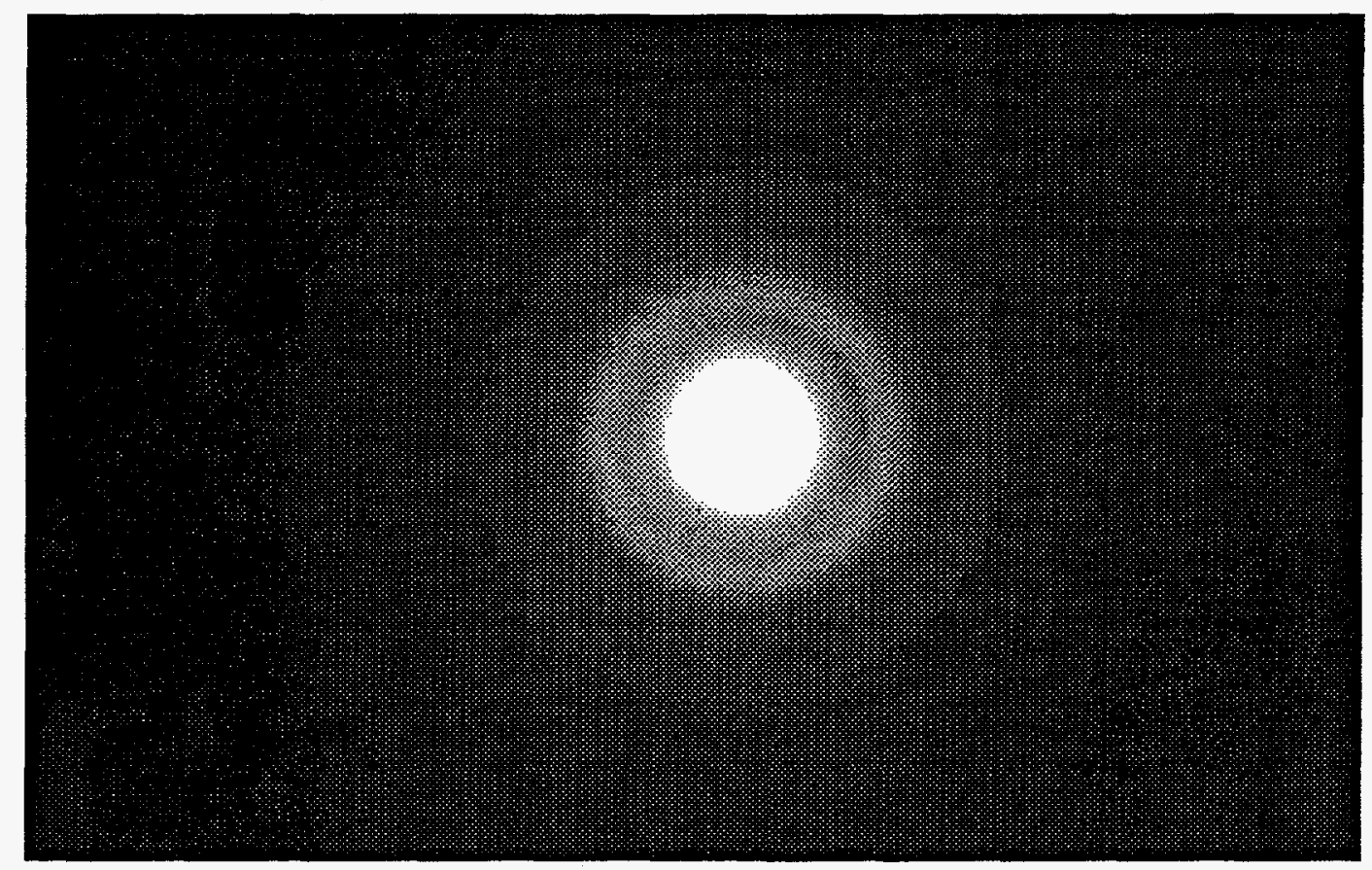

Figure 4.4. Selected area diffraction pattern of the modified layers for $4 \times 10^{17} \mathrm{C}^{+}$ions $/ \mathrm{cm}^{2}$. 


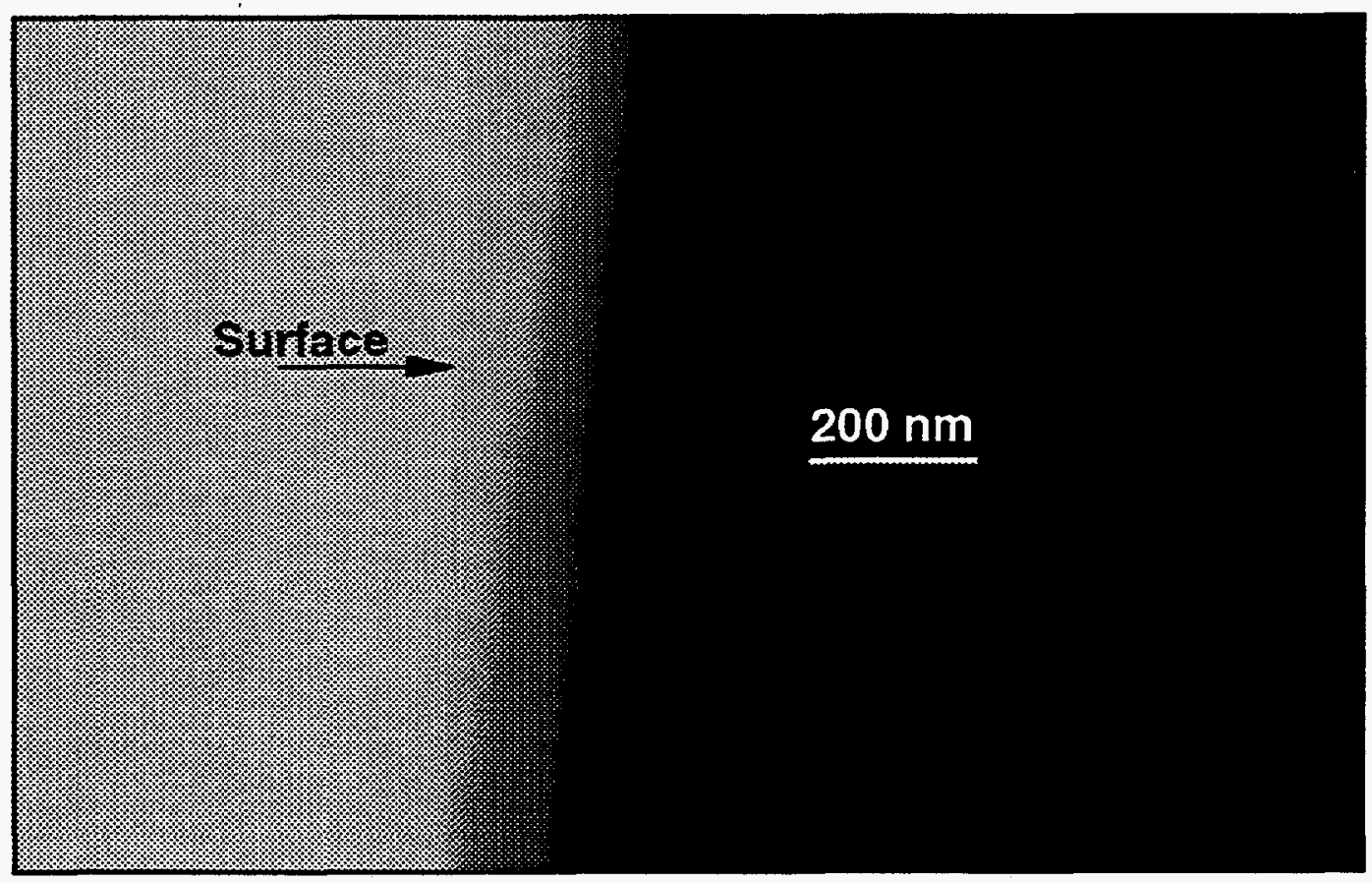

Figure 4.5. TEM bright-field image of $4 \times 10^{17} \mathrm{~N}^{+}$ions $/ \mathrm{cm}^{2}$ 


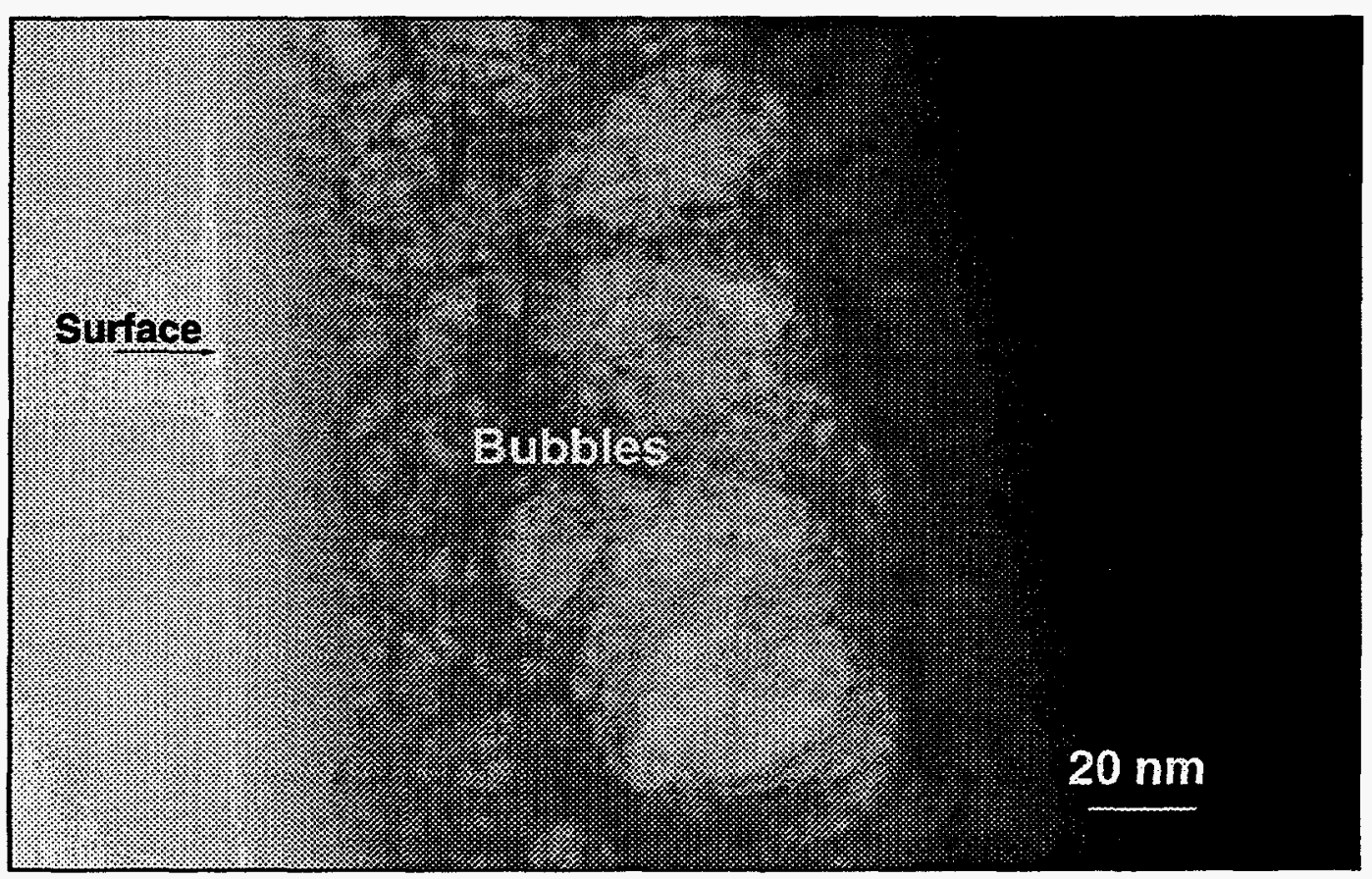

Figure 4.6. TEM bright-field image of $8 \times 10^{17} \mathrm{~N}^{+}$ions $/ \mathrm{cm}^{2}$. 


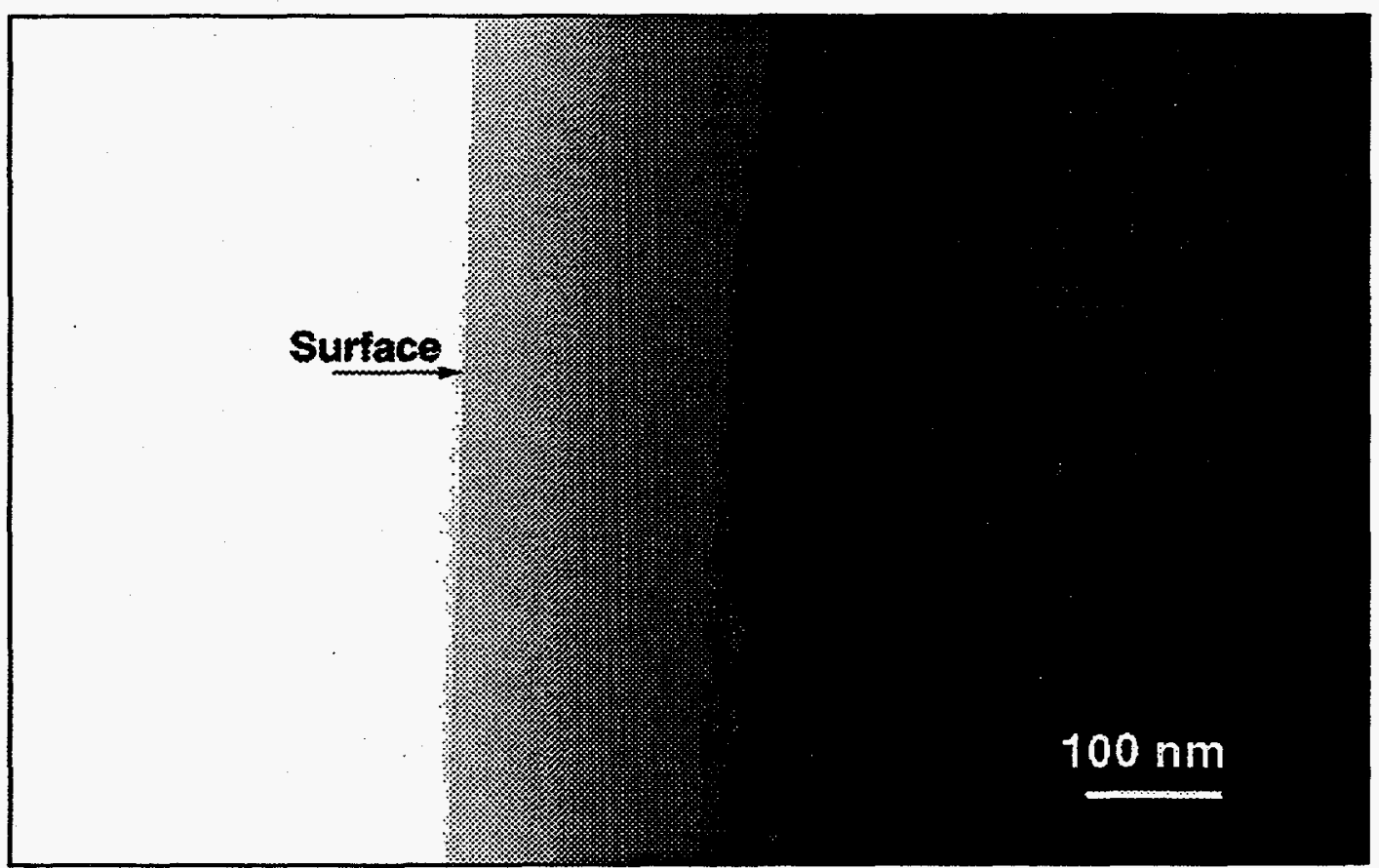

Figure 4.7. TEM bright-field image of $8 \times 10^{17} \mathrm{C}^{+}$ions $/ \mathrm{cm}^{2}$. 


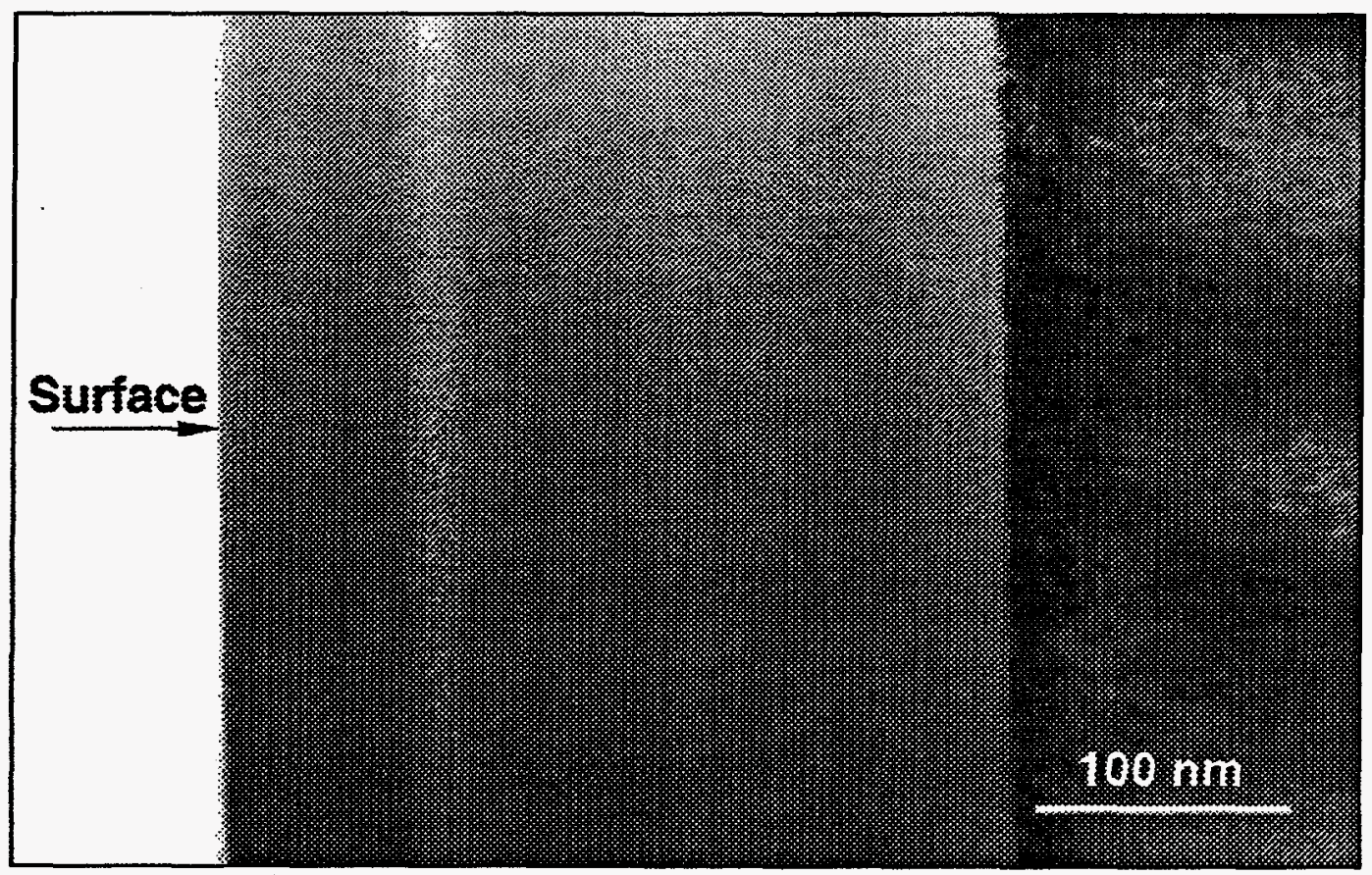

Figure 4.8. TEM bright-field image of $4 \times 10^{17} \mathrm{C}^{+}$ions $/ \mathrm{cm}^{2}+4 \times 10^{17} \mathrm{~N}^{+}$ions $/ \mathrm{cm}^{2}$. 
dose where the solubility of nitrogen in silicon was exceeded, nitrogen ions that were trapped formed gas bubbles, whereas carbon ions might have precipitated forming bonds with other carbon ions.

The bright-field image of the sample implanted with the mixed dose of $4 \times 10^{17}$ $\mathrm{C}^{+}$ions $/ \mathrm{cm}^{2}+4 \times 10^{17} \mathrm{~N}^{+}$ions $/ \mathrm{cm}^{2}$ indicated less dense material at approximately $85 \mathrm{~nm}$ from the surface (Figure 4.8). RBS data also indicated a nitrogen peak at $75 \mathrm{~nm}$ with an atomic fraction of nitrogen of about 0.6. The TEM and RBS measured ranges for nitrogen were in good agreement. At this projected range, the concentration of the implanted nitrogen ions might have exceeded the stochiometric composition of silicon nitride. As in the case of $8 \times 10^{17} \mathrm{~N}^{+}$ions $/ \mathrm{cm}^{2}$, the peak atomic fraction of nitrogen might have exceeded the solubility limit to form less dense material at the projected range in the material. The width of the modified layers calculated from the bright-field images are summarized in Table 4.6.

Table 4.6 Width of the ion-modified layer from TEM measurements.

\begin{tabular}{|l|c|}
\hline \multicolumn{1}{|c|}{ Sample } & $\begin{array}{c}\text { Width of the } \\
\text { ion modified layer } \\
\text { TEM measurements } \\
\text { (nm) }\end{array}$ \\
\hline Unimplanted & ---- \\
\hline $4 \times 10^{11} \mathrm{C}^{+}$ions $/ \mathrm{cm}^{2}$ & 257 \\
\hline $4 \times 10^{17} \mathrm{~N}^{+}$ions $/ \mathrm{cm}^{2}$ & 278 \\
\hline $4 \times 10^{17} \mathrm{C}^{+}$ions $/ \mathrm{cm}^{2}+4 \times 10^{17} \mathrm{~N}^{+}$ions $/ \mathrm{cm}^{2}$ & 314 \\
\hline $8 \times 10^{17} \mathrm{~N}^{+}$ions $/ \mathrm{cm}^{2}$ & --- \\
\hline $8 \times 10^{17} \mathrm{C}^{+}$ions/cm & 275 \\
\hline a: Includes implantation and radiation damage. \\
\hline
\end{tabular}

\subsection{Roughness Characterization}

Figures 4.9 a-f show roughness measurements obtained using AFM before and after implantation. Lighter regions correspond to a higher degree of elevation in these AFM figures. The RMS roughness of silicon before implantation of $0.14 \mathrm{~nm}$ changed to $0.21 \mathrm{~nm}$ when implanted with $4 \times 10^{17} \mathrm{C}^{+}$ions $/ \mathrm{cm}^{2}$. The RMS roughness 
determined from these measurements for the unimplanted and implanted samples at the start of the wear tests are summarized in Table 4.7 .

Table 4.7 Roughness values of the implanted and unimplanted $\mathrm{Si}(100)$ samples measured by AFM.

\begin{tabular}{|l|c|}
\hline \multicolumn{1}{|c|}{ Sample } & $\begin{array}{l}\text { RMS roughness in } \mathrm{nm} \\
\text { for a scan area } \\
2.5 \mu \mathrm{m} \times 2.5 \mu \mathrm{m}\end{array}$ \\
\hline Unimplanted & 0.14 \\
\hline $4 \times 10^{17} \mathrm{C}^{+}$ions $/ \mathrm{cm}^{2}$ & 0.21 \\
\hline $4 \times 10^{17} \mathrm{~N}^{+}$ions $/ \mathrm{cm}^{2}$ & 0.42 \\
\hline $4 \times 10^{17} \mathrm{C}^{+}$ions $/ \mathrm{cm}^{2}+4 \times 10^{17} \mathrm{~N}^{+}$ions $/ \mathrm{cm}^{2}$ & 0.91 \\
\hline $8 \times 10^{17} \mathrm{~N}^{+}$ions $/ \mathrm{cm}^{2}$ & 2.4 \\
\hline $8 \times 10^{17} \mathrm{C}^{+}$ions $/ \mathrm{cm}^{2}$ & 0.15 \\
\hline
\end{tabular}

\subsection{Discussion of Results}

The projected range and straggling were calculated from analytical equations, and computer simulations, and were measured by RBS. Analytical and computer simulations were based on the Gaussian shape of the implanted profile. The doses that were used in this study were high, and the implanted profile deviated from Gaussian. In the equation for $N_{p}, N_{p}=\frac{0.4 \phi_{i}}{\Delta R_{p}}$, the value $\phi_{i}$ was taken as the implanted dose in analytical and computer simulations. The estimated peak concentration and atomic 


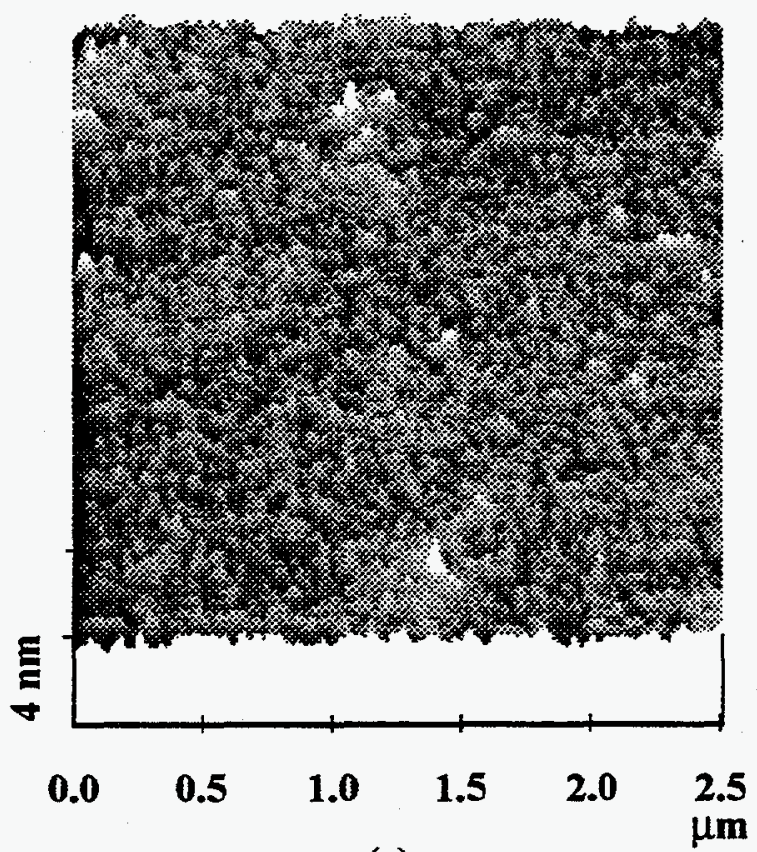

(a)

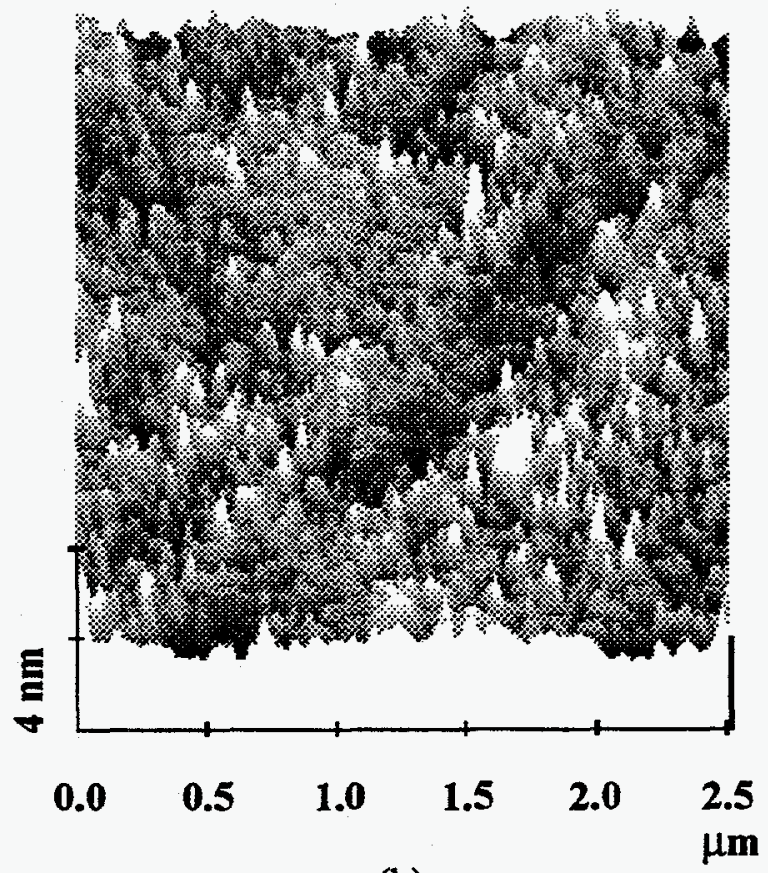

(b)

Figure 4.9 Atomic force microscopy images

(a) Unimplanted Silicon

(b) $4 \times 10^{17} \mathrm{C}^{+}$ions $/ \mathrm{cm}^{2}$ 


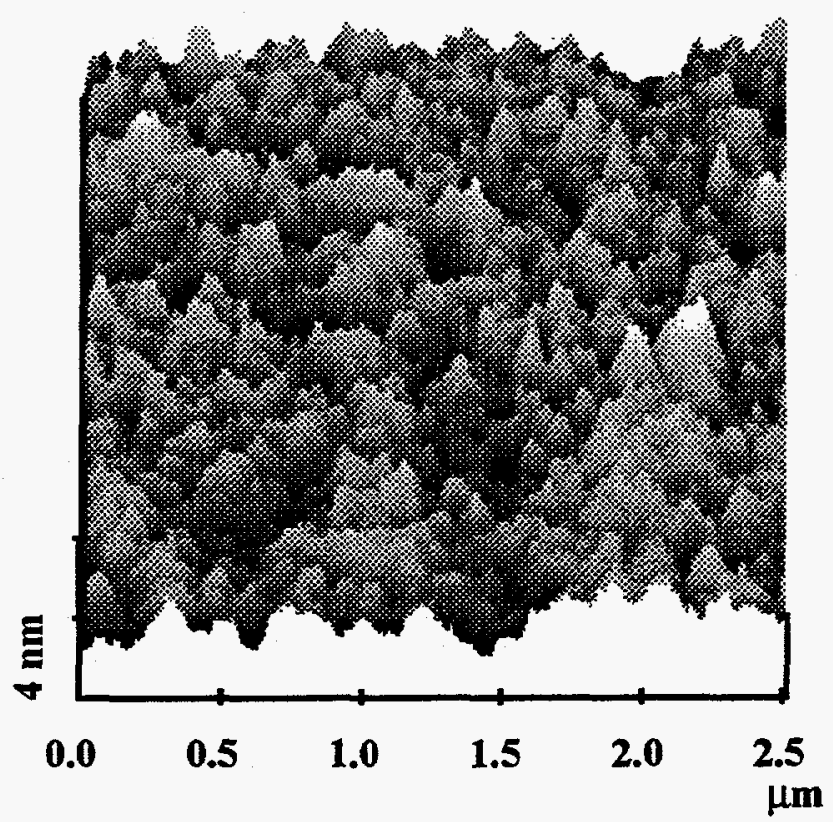

(c)

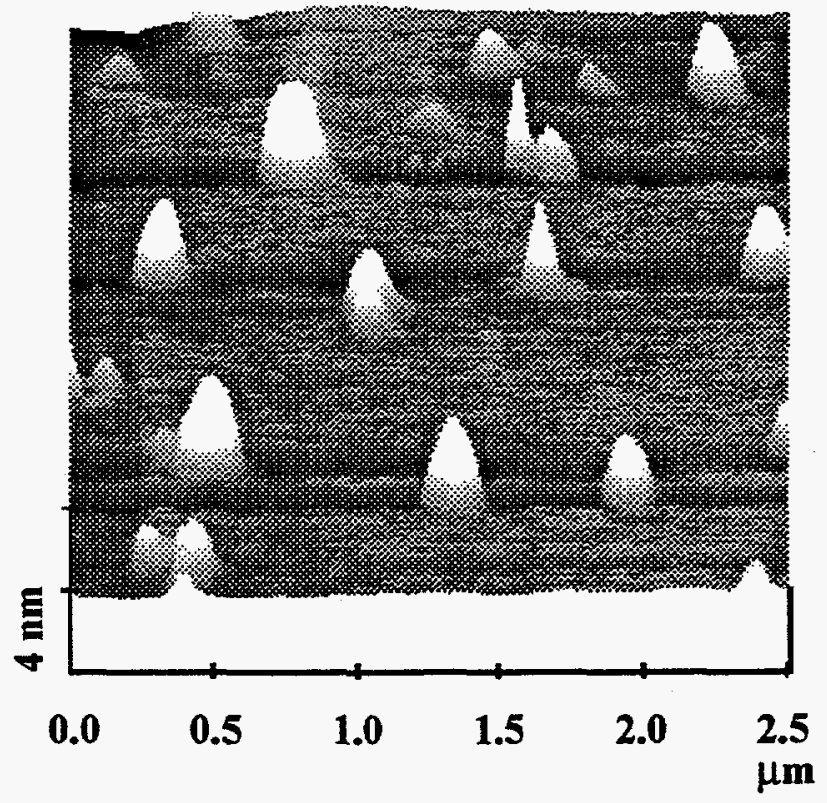

(d)

Figure 4.9 Atomic force microscopy images

(c) $4 \times 10^{17} \mathrm{C}^{+}$ions $/ \mathrm{cm}^{2}$

(d) $4 \times 10^{17} \mathrm{C}^{+}$ions $/ \mathrm{cm}^{2}+4 \times 10^{17} \mathrm{~N}^{+}$ions $/ \mathrm{cm}^{2}$ 


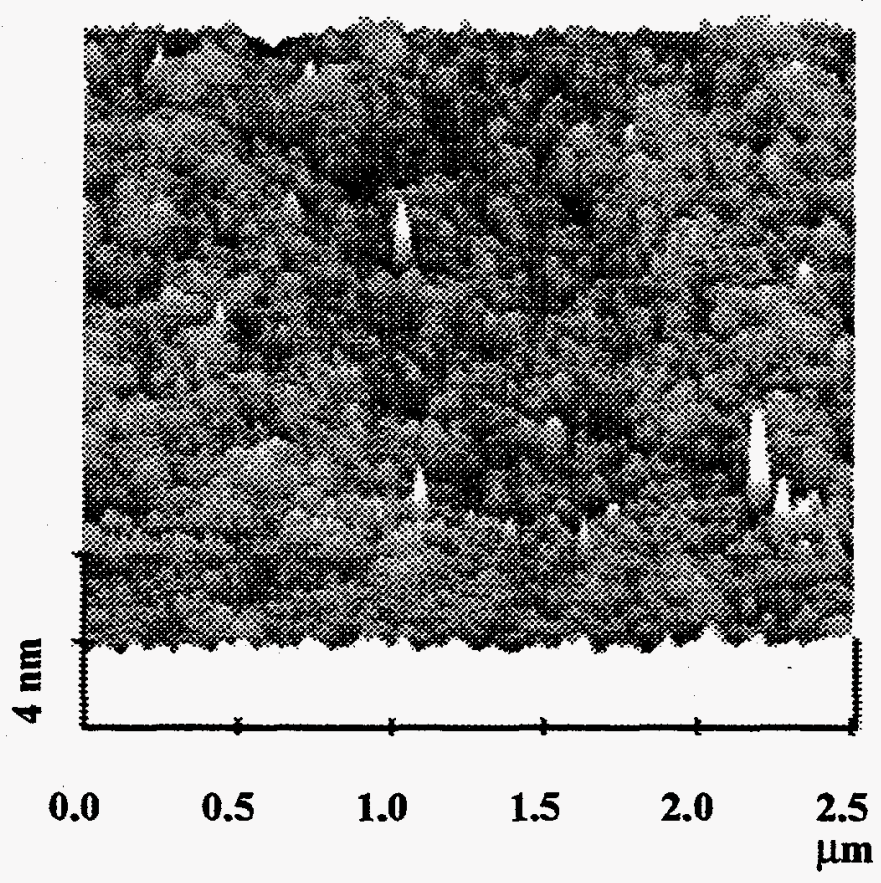

(e)

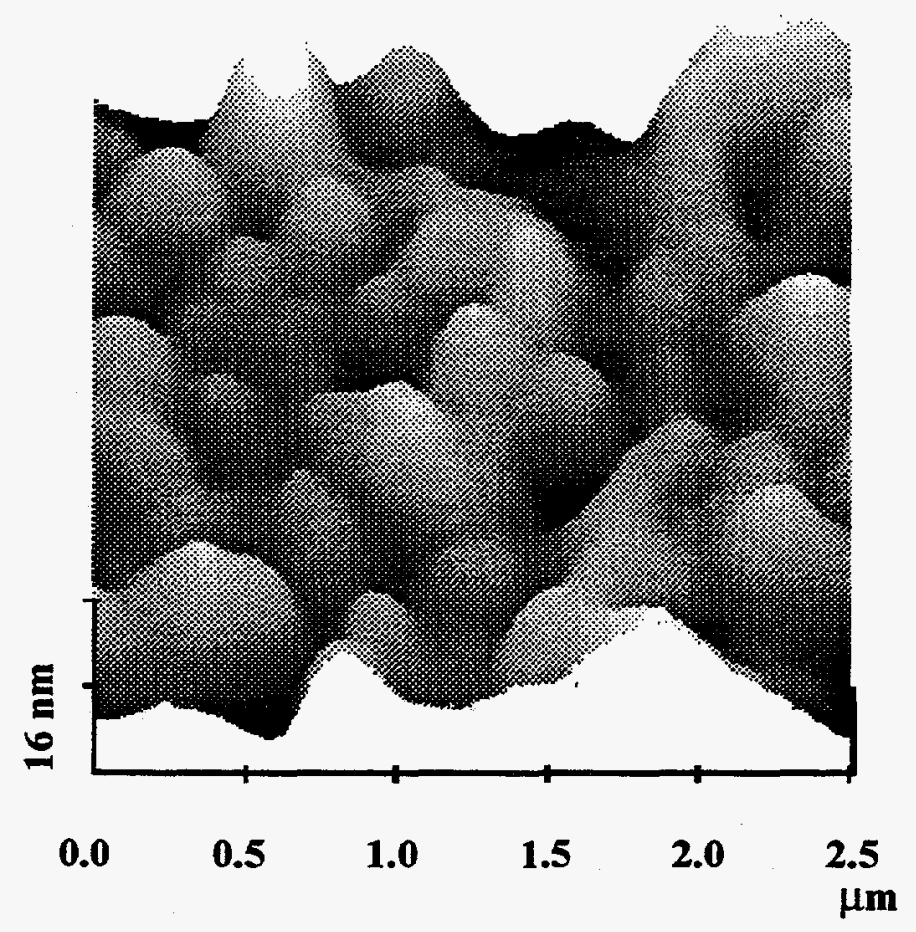

(f)

Figure 4.9 Atomic force microscopy images

(e) $8 \times 10^{17} \mathrm{C}^{+}$ions $/ \mathrm{cm}^{2}$

(f) $8 \times 10^{17} \mathrm{~N}^{+}$ions $/ \mathrm{cm}^{2}$ 
fraction of the implanted species predicted by analytical calculations were less than the measured values. These difference are due to the high estimated values of $\Delta R_{p}$ in the analytical approach.

The results obtained for the projected range of the implanted ions from analytical and TRIM calculations were in good agreement. For example, the projected range of carbon ions into silicon from TRIM calculations were $177.3 \mathrm{~nm}$, which is in reasonable agreement with the values obtained by analytical expressions; i.e., $183.7 \mathrm{~nm}$. Similarly, the analytical calculations of the projected range of nitrogen ions into silicon matched with TRIM calculations. However, the values for straggling of single implants of nitrogen and carbon ions into silicon from analytical expressions and TRIM calculations were in poor agreement, with TRIM values being less than the analytical calculated values.

Experimentally determined values for the projected range of carbon and nitrogen ions into silicon were in also in agreement with the values predicted by TRIM. For example, the projected range of carbon ions into silicon from TRIM calculations were $177.3 \mathrm{~nm}$, which was in reasonable agreement with the values obtained experimentally using RBS; namely, $180.0 \mathrm{~nm}$. The straggling predicted values by Monte Carlo simulations were within $35 \%$ of the experimentally determined values. As discussed by Nastasi et al. [16], simultaneous occurrence of sputtering, radiation enhanced diffusion, and compound formation with an increased dose could be responsible for the asymmetry of the implanted Gaussian profile. In addition, these phenomena can also lead to deviations from predictions and can result in higher straggling with increased dose. Thus, as expected, the experimentally measured straggling at higher doses $\left(8 \times 10^{17} \mathrm{C}^{+}\right.$ions $\left./ \mathrm{cm}^{2}\right)$ was $75.6 \mathrm{~nm}$, as compared to $39 \mathrm{~nm}$ at a lower dose $\left(4 \times 10^{17} \mathrm{C}^{+}\right.$ ions $/ \mathrm{cm}^{2}$ ).

The atomic concentration of carbon and nitrogen in the mixed implant $(4 \mathrm{C}+4 \mathrm{~N})$, were higher than observed at the lower dose of single implants of carbon ions (4C) or 
nitrogen ions $(4 \mathrm{~N})$. There is no simple method to determine the density and its variation throughout the modified layer. In this data analysis, the density of the modified layer was assumed to be uniform and could give rise to a higher measured peak atomic concentration than actually present.

The TEM bright-field image of the sample implanted with $4 \times 10^{17} \mathrm{~N}^{+}$ions $/ \mathrm{cm}^{2}$ indicated that the modified layer was amorphous. The measured atomic concentration of nitrogen ions was $73 \%$ when the dose was increased to $8 \times 10^{17} \mathrm{~N}^{+}$ions $/ \mathrm{cm}^{2}$. This fraction exceeds the concentration of nitrogen ions that is needed to form stoichoimetric silicon nitride. The excess nitrogen ions formed bubbles throughout the entire modified region (Figure 4.6). These bubbles were due to coalescence of nitrogen molecules that were formed when $\mathrm{N}-\mathrm{N}$ bonds reached a critical density. Kinetics and thermodynamics of the bubble formation are discussed in the next chapter. Although the concentration of carbon ions that is needed to form stoichiometric silicon carbide was exceeded for the sample implanted with $8 \times 10^{17} \mathrm{C}^{+}$ions $/ \mathrm{cm}^{2}$, no bubbles were observed (Figure 4-7). At a high dose of nitrogen the solubility of nitrogen in silicon was exceeded. Nitrogen ions that were trapped formed gas bubbles. In contrast, at a high dose of carbon ions, the excess carbon ions may have precipitated forming bonds with other carbon ions. Thus, small regions of graphite or diamond could be found in the sample implanted with high dose of carbon ions.

The bright-field image of the sample implanted with the mixed dose of $4 \times 10^{17}$ $\mathrm{C}^{+}$ions $/ \mathrm{cm}^{2}+4 \times 10^{17} \mathrm{~N}^{+}$ions $/ \mathrm{cm}^{2}$ indicated less dense material at approximately $85 \mathrm{~nm}$ from the surface (Figure 4.8). This distance corresponded to the measured depth of the projected range of nitrogen ions in carbon-implanted silicon measured by RBS. At this depth the peak atomic fraction might be slightly greater than 0.57 . The concentration of $\mathrm{N}-\mathrm{N}$ bonds might not have been sufficient to form bubbles.

The width of the damaged layer measured by TEM studies (Table 4.6) was compared with the width of the ion-modified layer determined by the channeling data 
(Table 4.5). Agreement between the two measurements was good. For example, the width of the modified layer for a sample implanted with $4 \times 10^{17} \mathrm{C}^{+}$ions $/ \mathrm{cm}^{2}$.measured by channeling experiments was $300 \mathrm{~nm}$, whereas TEM measurements revealed the modified layer to be $257 \mathrm{~nm}$. The slight difference in the thickness values arose from not taking atomic density changes into account when estimating thickness from the channeling data.

AFM measurements revealed changes in the surface roughness of the implanted samples. Sputtering and formation of new compounds may have contributed to these changes in the roughness.

\subsection{Conclusions}

When the projected range and straggling obtained from analytical equations, computer simulations, and RBS data, were compared, the limitation of these methods became evident. For example, the range estimated by analytical equations, computer simulations, and RBS data are in agreement. However, the straggling estimated by the analytical expressions is twice the value obtained by computer simulations. The analytical expressions were derived for a constant homogenous composition target, single-implantation event and do not account for sputtering. It was also found that by using analytical expressions, the projected range, $R_{p}$, and straggling, $\Delta R_{p}$, for mixedimplant specimens could not be estimated.

The analytical equations and ion beam analysis assumed a Gaussian distribution of an implanted profile. The implantation doses used in the present experiments were high. At these high doses $\left(4 \times 10^{17}\right.$ and $8 \times 10^{17}$ ions $\left./ \mathrm{cm}^{2}\right)$, the implantation profile deviated from the Gaussian distribution. Similar to the analytical expressions, the ion beam analysis also assumed the density of the modified layer to be uniform. However, the changes in the composition that arise from the implantation could give rise to higher measured peak atomic concentration than actually present. In the Monte Carlo simulations, the results were averaged over many simulated particle trajectories. 
TEM studies revealed that implantation of carbon and nitrogen ions at doses of $4 \times 10^{17}$ and $8 \times 10^{17}$ ions $/ \mathrm{cm}^{2}$ into $\mathrm{Si}(100)$ produced an amorphous layer. The width of the modified layer determined by TEM and channeling measurements were in agreement. The studies also indicated that there was a limit on the solid solubility for nitrogen ions at room temperature. TEM data indicated bubble formation in the sample implanted with a dose of $8 \times 10^{17} \mathrm{~N}^{+}$ions $/ \mathrm{cm}^{2}$. For the sample implanted with $8 \times 10^{17} \mathrm{C}^{+}$ ions $/ \mathrm{cm}^{2}$, no bubbles were observed.

In summary, implantation of carbon and nitrogen ions at doses of $4 \times 10^{17}$ ions $/ \mathrm{cm}^{2}$ and $8 \times 10^{17}$ ions $/ \mathrm{cm}^{2}$ into $\mathrm{Si}(100)$ produced an amorphous layer. The peak atomic concentration of the implanted species increased with dose. The width of the modified layer, determined by TEM and channeling measurements were in agreement. 


\section{Chapter 5 Mechanical and Tribological Properties of Implanted Silicon}

Ion implantation is useful for improving tribological properties because of its ability to precisely control the composition of the modified layer and to produce strong adhesion of the ion-implanted layer to the substrate. Applications and examples of the ion implantation process to modify the friction and wear properties of metals, metallic alloys, ceramics, and polymers [17-22] are discussed in Chapter 2.

There are several reports on the investigations of tribological properties of ionimplanted silicon in the literature [23-26]. J. Lekki et al. [23] implanted argon ions into silicon, and their data suggests that no chemical change was required in the modified layer to improve friction and wear properties. They suggested that the defects created during the implantation propagated below the surface during the wear process and helped reduce friction. Carbon-implantation studies on silicon by Miyamoto et al. [25] indicated that the wear resistance of implanted silicon was greater than that of unimplanted silicon. XPS studies revealed that the structure of the carbon implanted region was similar to that of SiC. Gupta and Bhushan [26] studied the friction and wear behavior effect of carbon and nitrogen implantation into single-crystal and polycrystalline silicon as a function of dose and energy. Their studies indicated a change in the chemistry of the near-surface region that improved the tribological properties of silicon. There are no reports on mixed implantation of carbon and nitrogen ions into silicon.

\subsection{Objective}

The objective of this research was to investigate whether mixed implantation can be effective in improving the tribological properties of single crystal silicon. In light of the general correlation between the hardness and wear resistance discussed in Chapter 2 
(Eqn. [2.4] ), creating a harder phases such as silicon carbide or silicon nitride may improve its tribological properties. Silicon carbide and silicon nitride have high hardness and good wear resistance [54]. Thus, two possible species that can be implanted into silicon are carbon and nitrogen ions. This study explored the promising materials that may result from the mixed implantation of carbon and nitrogen ions and an understanding of the tribological performance of silicon implanted with both carbon and nitrogen ions along with the single implants of carbon and nitrogen.

The experimental details and results on the characterization of carbon and nitrogen ions into single crystal silicon were discussed in the previous chapter. Some of the results obtained in the previous chapter, that will be used in this chapter are recapitulated here. Implantation of carbon and nitrogen ions at a doses of $4 \times 10^{17}$ and $8 \times 10^{17}$ ions $/ \mathrm{cm}^{2}$ into $\mathrm{Si}(100)$ produced an amorphous layer. The TEM bright-field image of the sample implanted with $8 \times 10^{17} \mathrm{~N}^{+}$ions $/ \mathrm{cm}^{2}$ indicated a low-density region that was caused by nitrogen ions trapped in the form of bubbles in the entire modified region (Figure 4.6). RBS studies indicated that the peak atomic fraction of the nitrogen ions was approximately 0.47 and 0.72 in the samples implanted with $4 \times 10^{17} \mathrm{~N}^{+}$ions $/ \mathrm{cm}^{2}$ and $8 \times 10^{17} \mathrm{~N}^{+}$ions $/ \mathrm{cm}^{2}$, respectively. The peak concentration also increased with dose for carbon implanted samples. For the sample implanted with $8 \times 10^{17} \mathrm{C}^{+}$ions $/ \mathrm{cm}^{2}$, the peak concentration was greater than the amount (50\%) required to form stoichiometric silicon carbide.

\subsection{Results}

The results of the investigations of the mechanical and tribological properties of the implanted silicon are presented in the following order.

1) Surface hardness

2) Tribological properties

3) Discussion of results 


\subsubsection{Surface Hardness Measurements}

Surface hardness of the unimplanted samples and the implanted sample was measured using a nanoindenter. Figures 5.1 and 5.2 show the hardness, measured using a nanoindenter, as a function of penetration depth. Table 5.1 summarizes the hardness and modulus of the unimplanted and implanted $\mathrm{Si}(100)$ at a depth of $57 \mathrm{~nm}$. The width of the modified layer, as determined from TEM measurements for different samples, varied from $250 \mathrm{~nm}$ to $315 \mathrm{~nm}$. Thus, to minimize substrate effects, the depth at which the hardness is presented, $57 \mathrm{~nm}$, is only a fraction of the modified layer.

The hardness of the sample implanted with $4 \times 10^{17} \mathrm{~N}^{+}$ions $/ \mathrm{cm}^{2}$ was found to be almost the same as the unimplanted sample (Figure 5.1). However, with an increase in dose $\left(8 \times 10^{17} \mathrm{~N}^{+}\right.$ions $\left./ \mathrm{cm}^{2}\right)$, a decrease in hardness relative to the unimplanted sample was observed (Figure 5.1). 


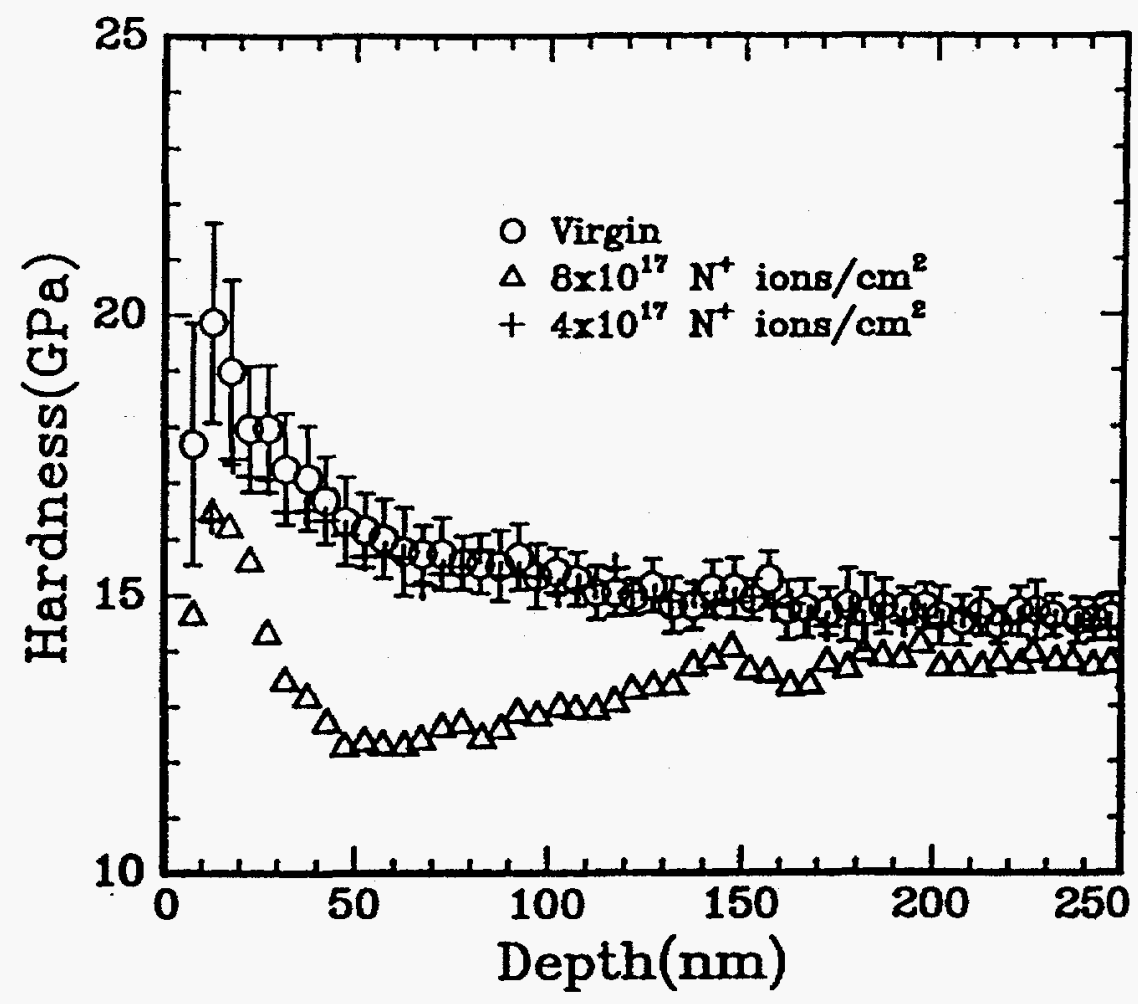

Figure 5.1 Surface hardness as a function of depth for nitrogen-implanted samples. 
In the carbon-implanted samples, hardness increased by $6 \%$ at low dose $\left(4 \times 10^{17}\right.$ $\mathrm{C}^{+}$ions $\left./ \mathrm{cm}^{2}\right)$ and by $30 \%$ at a higher dose $\left(8 \times 10^{17} \mathrm{C}^{+}\right.$ions $\left./ \mathrm{cm}^{2}\right)$ relative to the unimplanted sample (Figure 5.2).

Surface hardness of the samples implanted with $4 \times 10^{17} \mathrm{C}^{+}$ions $/ \mathrm{cm}^{2}$ and $4 \times 10^{17}$ $\mathrm{N}^{+}$ions $/ \mathrm{cm}^{2}$ were $17.3 \mathrm{GPa}$ and $15.7 \mathrm{GPa}$, respectively. However, the hardness of sample implanted with both $4 \times 10^{17} \mathrm{C}^{+}$ions $/ \mathrm{cm}^{2}$ and $4 \times 10^{17} \mathrm{~N}^{+}$ions $/ \mathrm{cm}^{2}$ was found to be 21.8 GPa. The magnitude of the hardness after a mixed implant is nearly same as the hardness measured from the sample implanted with $8 \times 10^{17} \mathrm{C}^{+}$ions $/ \mathrm{cm}^{2}$, as shown in Figure 5.2.

\subsubsection{Tribological Studies}

Friction and wear tests were performed using a pin-on-disc (POD) apparatus with a AISI52100 steel pin under contact stress of $225 \mathrm{MPa}$ and at $35 \%$ relative humidity. The contact stress, $225 \mathrm{MPa}$, was less than the yield strength of silicon, 5 $\mathrm{GPa}$, thus minimizing wear by plastic deformation. Friction data of the unimplanted sample and the implanted samples are shown in Figures 5.3 and 5.4. The sample implanted with $4 \times 10^{17} \mathrm{~N}^{+}$ions $/ \mathrm{cm}^{2}$ showed a slight decrease in the coefficient of friction relative to the unimplanted sample. However, the sample implanted with $8 \times 10^{17} \mathrm{~N}^{+}$ ions $/ \mathrm{cm}^{2}$ showed an increase in the coefficient of friction.

The carbon-implanted samples $\left(4 \times 10^{17} \mathrm{C}^{+}\right.$ions $/ \mathrm{cm}^{2}$ and $8 \times 10^{17} \mathrm{C}^{+}$ions $\left./ \mathrm{cm}^{2}\right)$ showed a low coefficient of friction for about 300 cycles and then increased to the value of the unimplanted sample (0.6). The sample implanted with both carbon and 


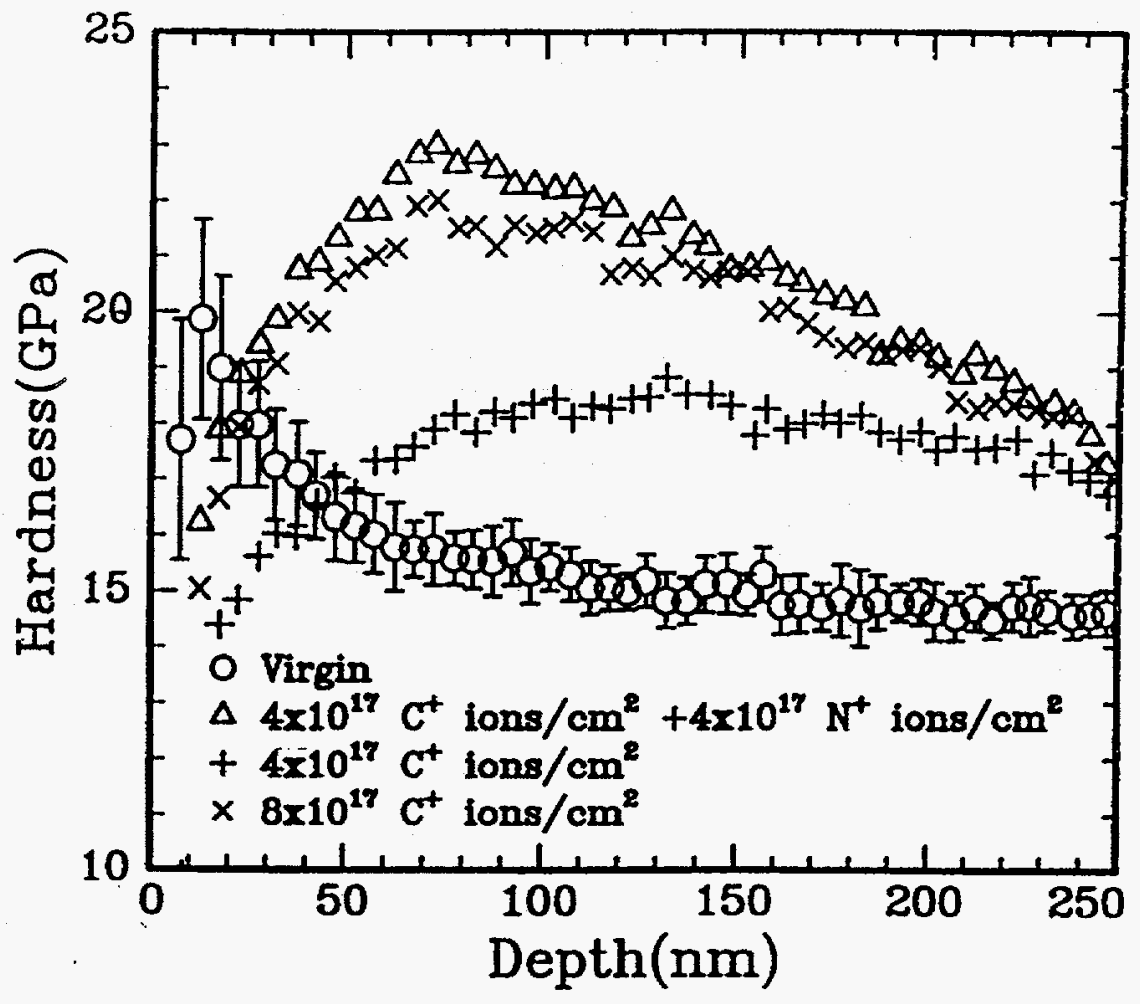

Figure 5.2 Surface hardness as a function of depth for carbon-implanted samples. 
Table 5.1 Hardness, modulus, and wear factor of the unimplanted and implanted $\mathrm{Si}(100)$.

\begin{tabular}{|l|c|c|c|}
\hline \multicolumn{1}{|c|}{ Sample } & $\begin{array}{c}\text { Hardness } \\
\text { GPa }\end{array}$ & $\begin{array}{c}\text { Elastic Modulus } \\
\text { GPa }\end{array}$ & $\begin{array}{c}\text { Wear Factor for } 660 \\
\text { Cycles } \\
\left(10^{-6} \mathrm{~mm}^{3} / \mathrm{N} . \mathrm{m}\right)\end{array}$ \\
\hline Unimplanted & $16.0 \pm 0.7$ & $191.0 \pm 9.0$ & 229.7 \\
\hline $4 \times 10^{17} \mathrm{C}^{+}$ions $/ \mathrm{cm}^{2}$ & $17.3 \pm 1.1$ & $200.6 \pm 8.9$ & 103.4 \\
\hline $4 \times 10^{17} \mathrm{~N}^{+}$ions $/ \mathrm{cm}^{2}$ & $15.7 \pm 0.9$ & $188.3 \pm 8.3$ & 252.1 \\
\hline $\begin{array}{l}4 \times 10^{17} \mathrm{C}^{+} \text {ions } / \mathrm{cm}^{2}+ \\
4 \times 10^{17} \mathrm{~N}^{+} \text {ions } / \mathrm{cm}^{2}\end{array}$ & $21.8 \pm 1.0$ & $217.5 \pm 7.0$ & 162.1 \\
\hline $8 \times 10^{17} \mathrm{~N}^{+}$ions $/ \mathrm{cm}^{2}$ & $12.3 \pm 0.8$ & $155.0 \pm 0.6$ & Scattered \\
\hline $8 \times 10^{17} \mathrm{C}^{+}$ions $/ \mathrm{cm}^{2}$ & $21.0 \pm 1.0$ & $219.6 \pm 8.7$ & 67.1 \\
\hline *: Measurements taken at a depth of $57 \mathrm{~nm}$ \\
\hline
\end{tabular}




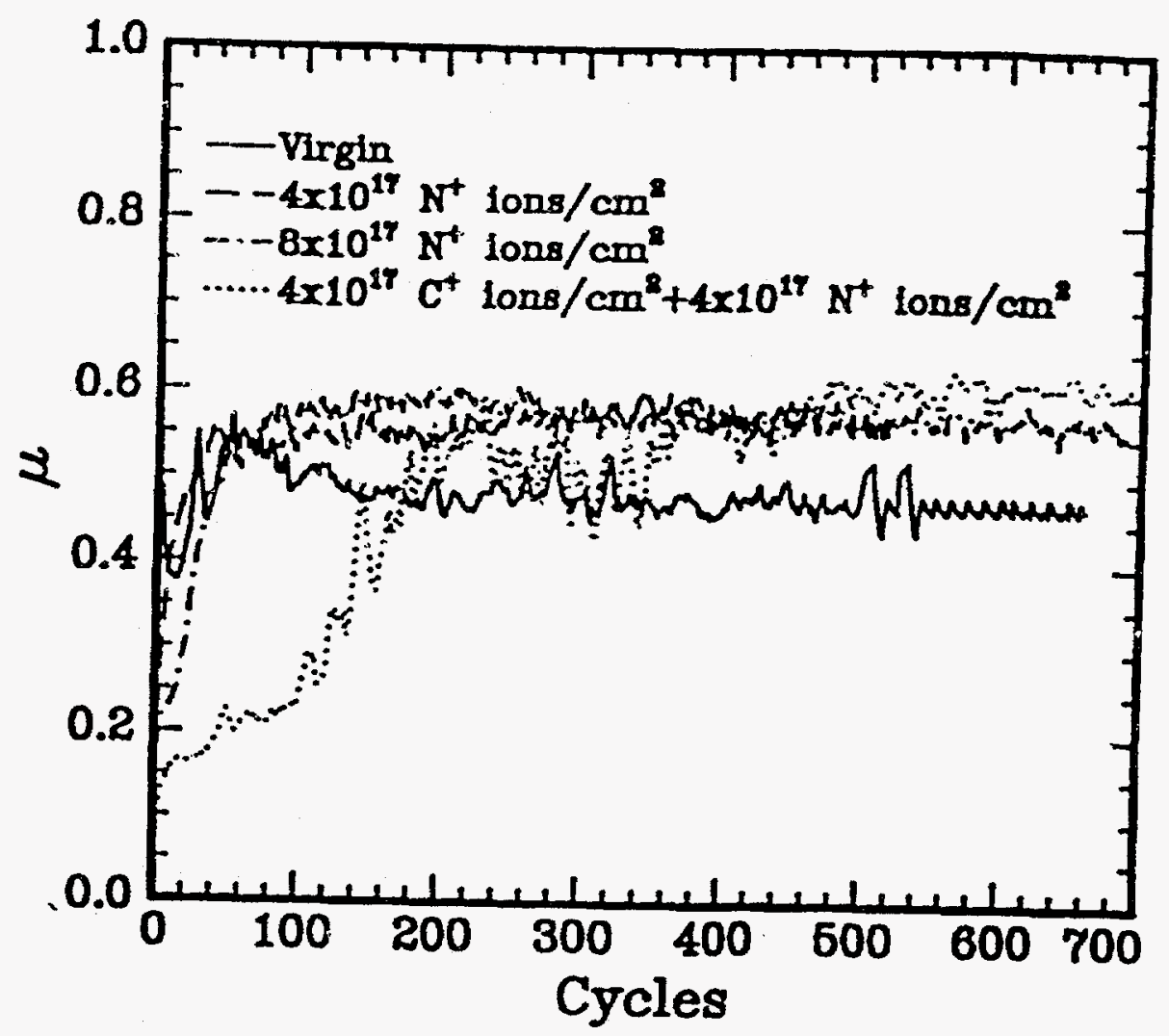

Figure 5.3 Coefficient of friction as a function of cycles for nitrogen-implanted samples. 


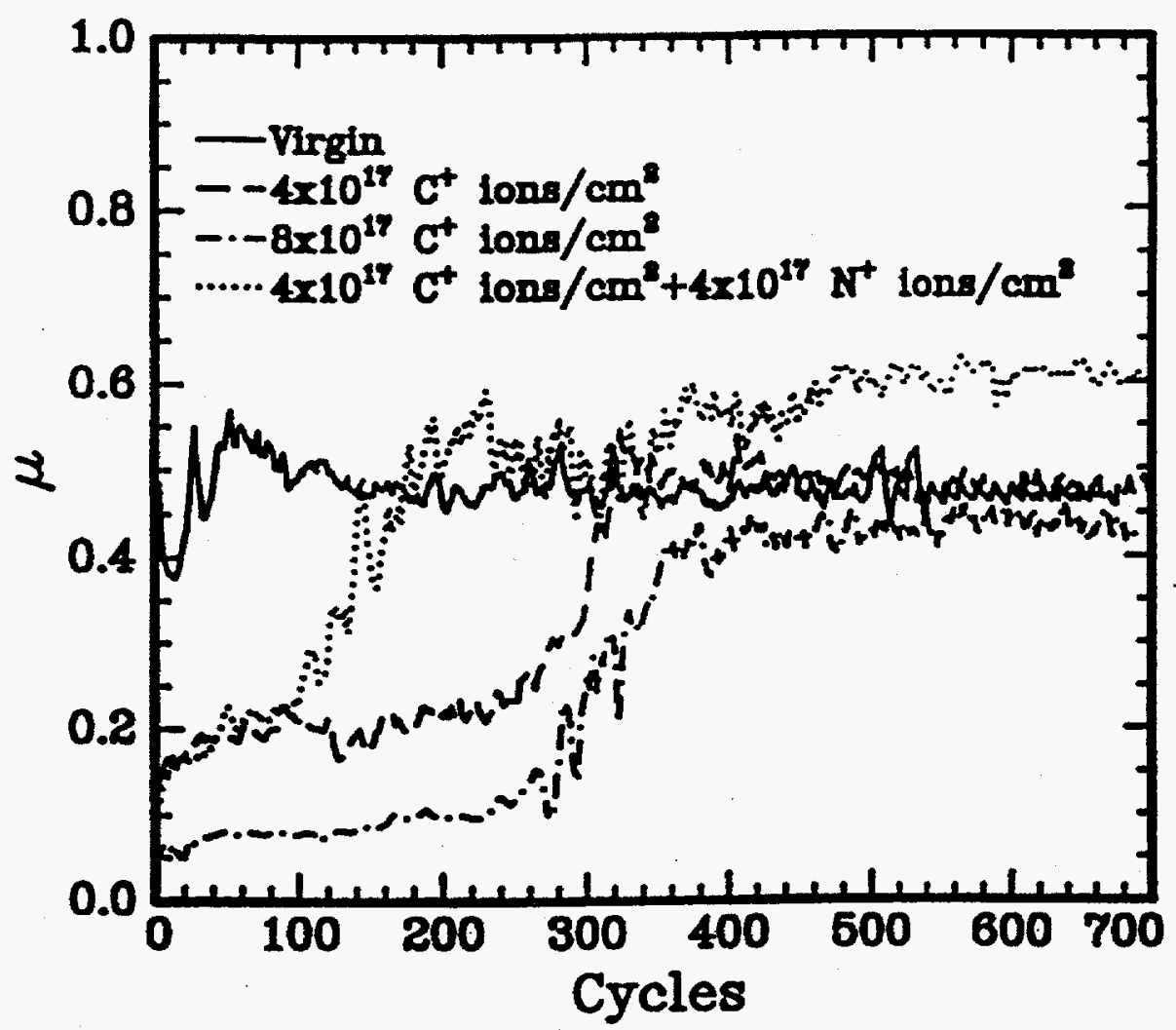

Figure 5.4 Coefficient of friction as a function of cycles for carbon-implanted samples. 
nitrogen $\left(4 \times 10^{17} \mathrm{C}^{+}\right.$ions $/ \mathrm{cm}^{2}+4 \times 10^{17} \mathrm{~N}^{+}$ions $\left./ \mathrm{cm}^{2}\right)$ showed a low coefficient of friction for about 100 cycles.

The wear morphology of the samples implanted with $4 \times 10^{17} \mathrm{C}^{+}$ions $/ \mathrm{cm}^{2}$ and $8 \times 10^{17} \mathrm{~N}^{+}$ions $/ \mathrm{cm}^{2}$ after 150 cycles is shown in Figure 5.5. The morphology of the wear tracks after 660 cycles of sliding for a variety of implant conditions are shown in Figures 5.6 and 5.7. These scanning electron micrographs revealed parallel grooves running along the wear tracks and loose wear debris inside the track. Wear morphologies observed for 150 and 660 cycles were similar. In all the cases, no measurable wear was evident on the slider. A transfer of wear debris to the slider was observed; however, this wear debris was loose and easily removed by blowing gas.

The RMS roughness value of the 52100 steel pin before the wear test was $3 \mathrm{~nm}$. After the wear tests, no damage was observed on the pin. The contact radius of the pin on the silicon substrate for static loading conditions was calculated to be $30 \mu \mathrm{m}$. The roughness measurement of the pins at the end of the wear tests were difficult. Hence, as an upper boundary, after the wear tests, the RMS roughness of the pin was assumed to be close to the RMS roughness of the disc in the plasticity index calculations. The measured roughness values of the wear tracks are summarized in Table 5.2. These values of roughness were used to evaluate the plasticity index using Eqn. [2.1]. 


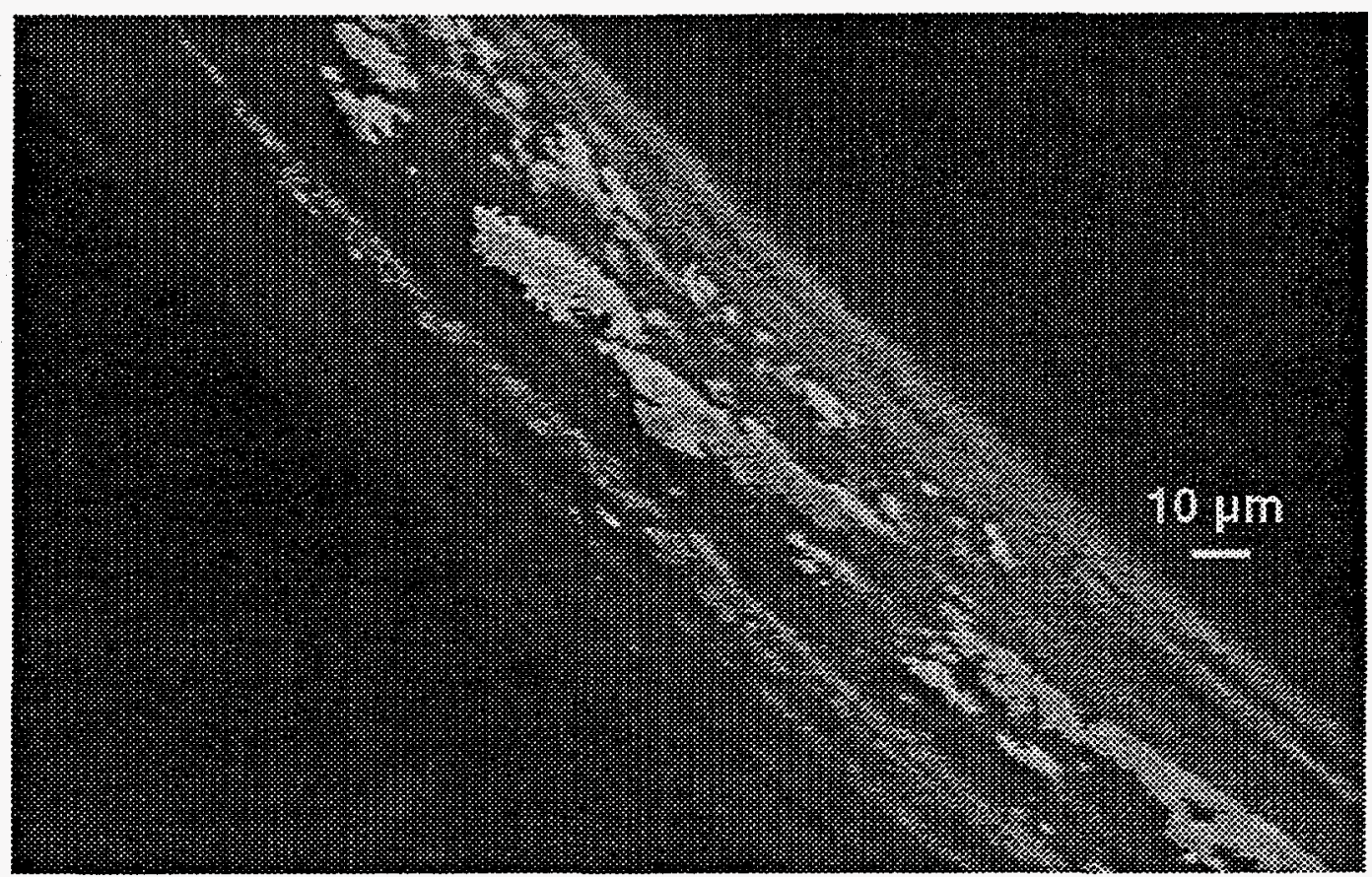

(a)

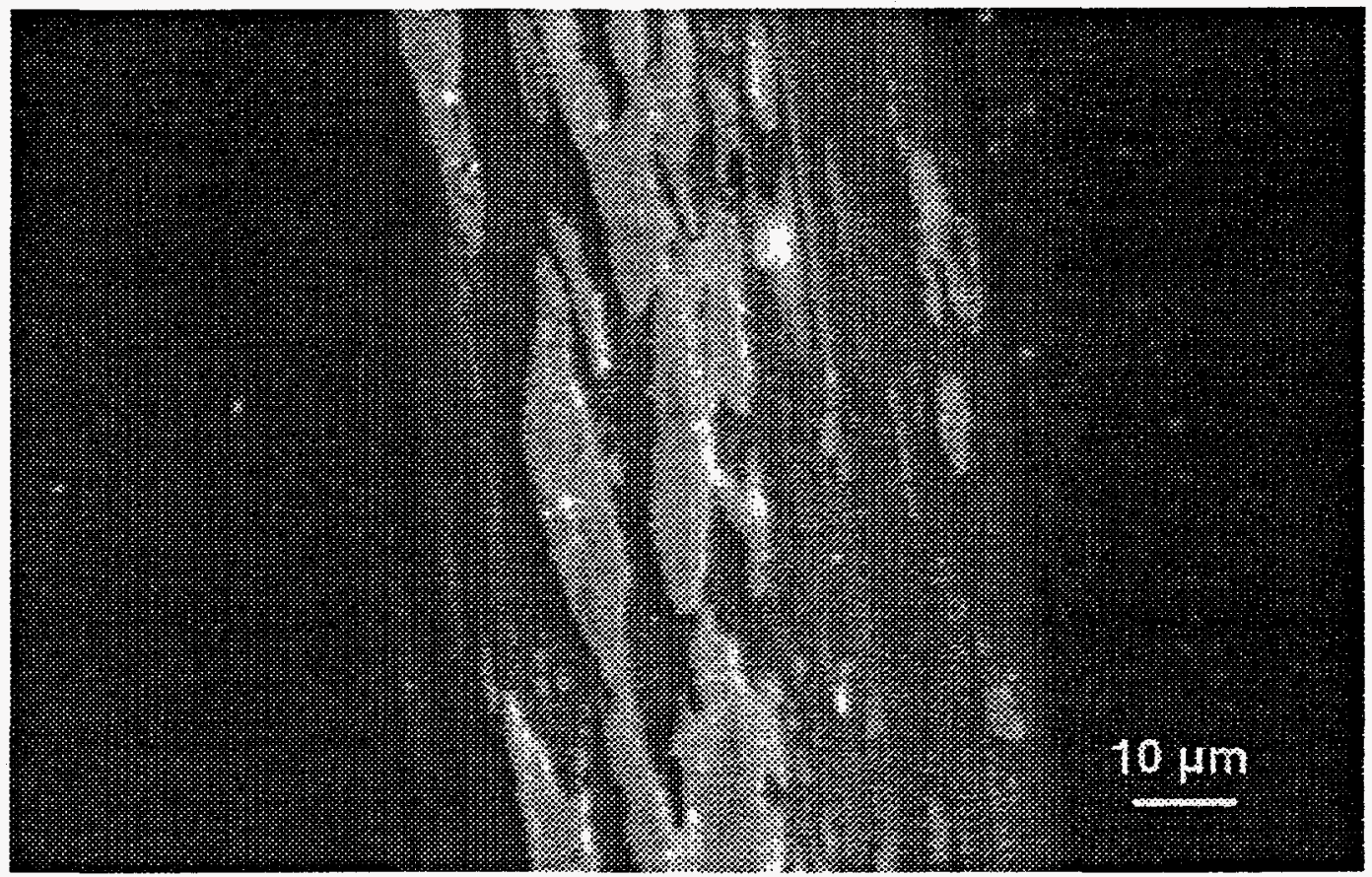

(b)

Figure 5.5. SEMs showing the morphology of the wear tracks after 150 cycles (a) $4 \times 10^{17} \mathrm{C}^{+}$ions $/ \mathrm{cm}^{2}$ (b) $8 \times 10^{17} \mathrm{~N}^{+}$ions $/ \mathrm{cm}^{2}$. 


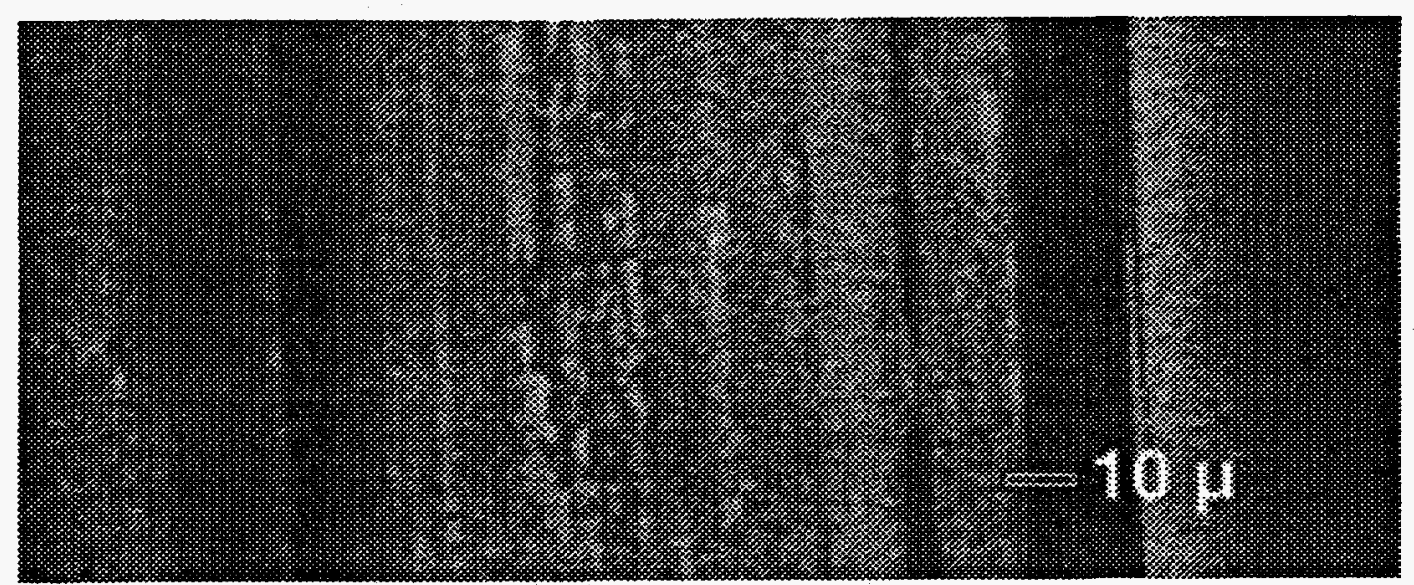

(a)

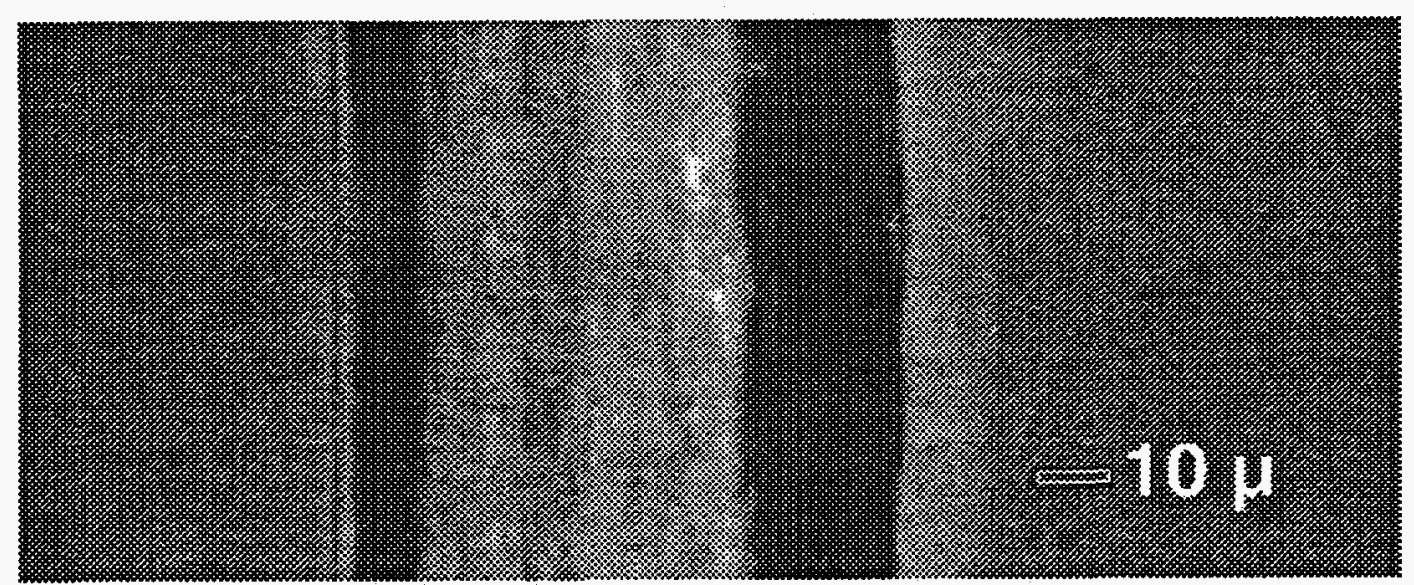

(b)

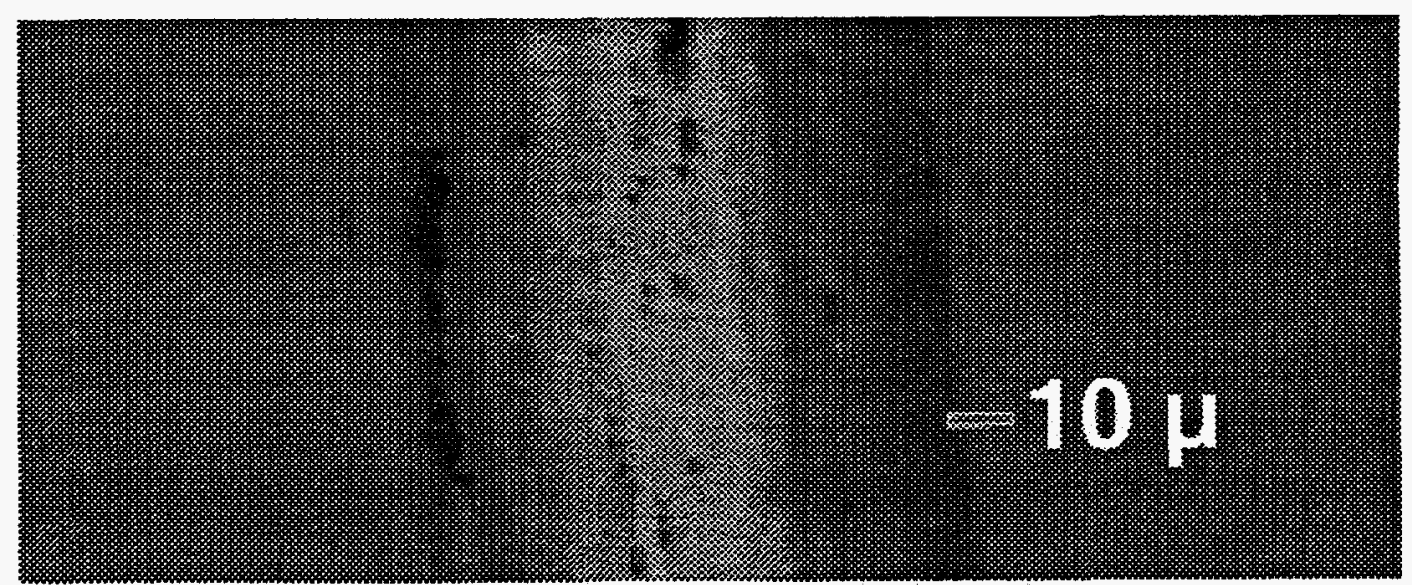

(c)

Figure 5.6. SEMs of the wear tracks after 660 cycles

(a) Unimplanted silicon (b) $4 \times 10^{17} \mathrm{C}^{+}$ions $/ \mathrm{cm}^{2}$

(c) $8 \times 10^{17} \mathrm{C}^{+}$ions $/ \mathrm{cm}^{2}$. 


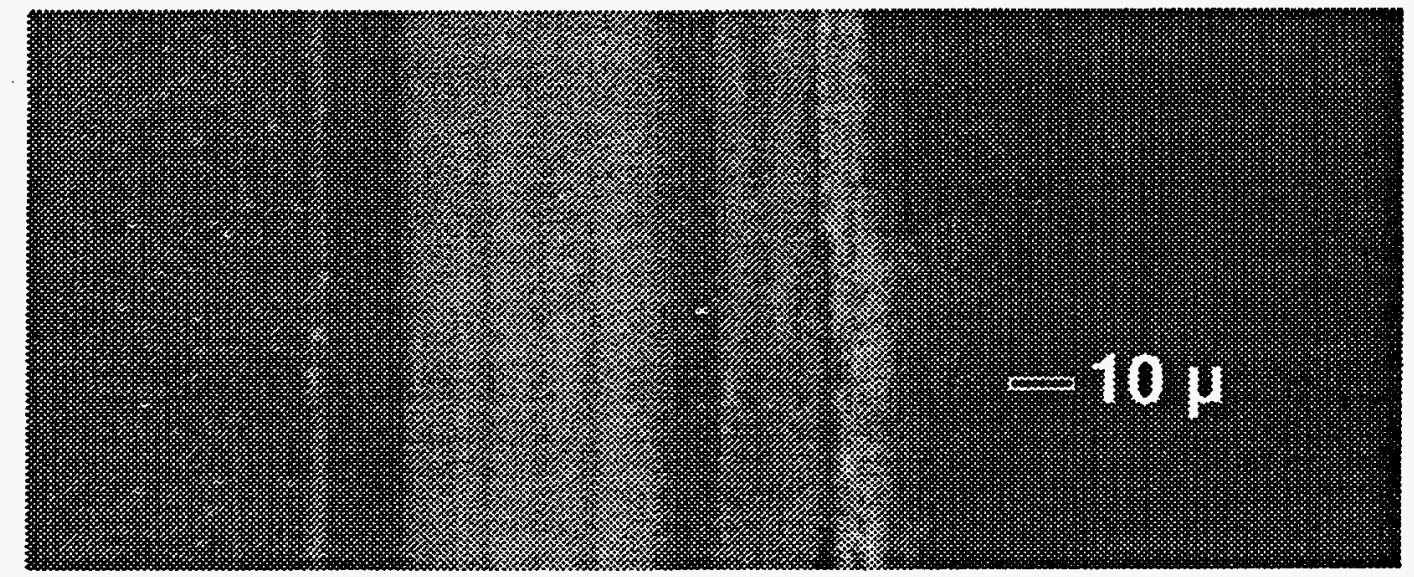

(a)

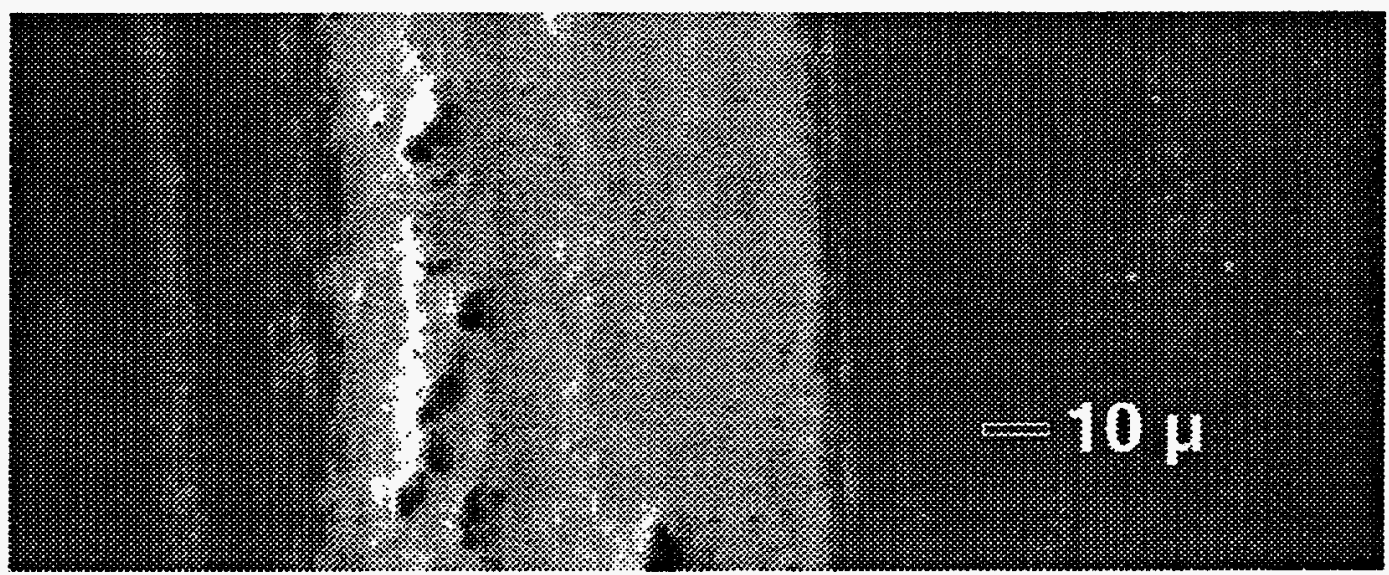

(b)

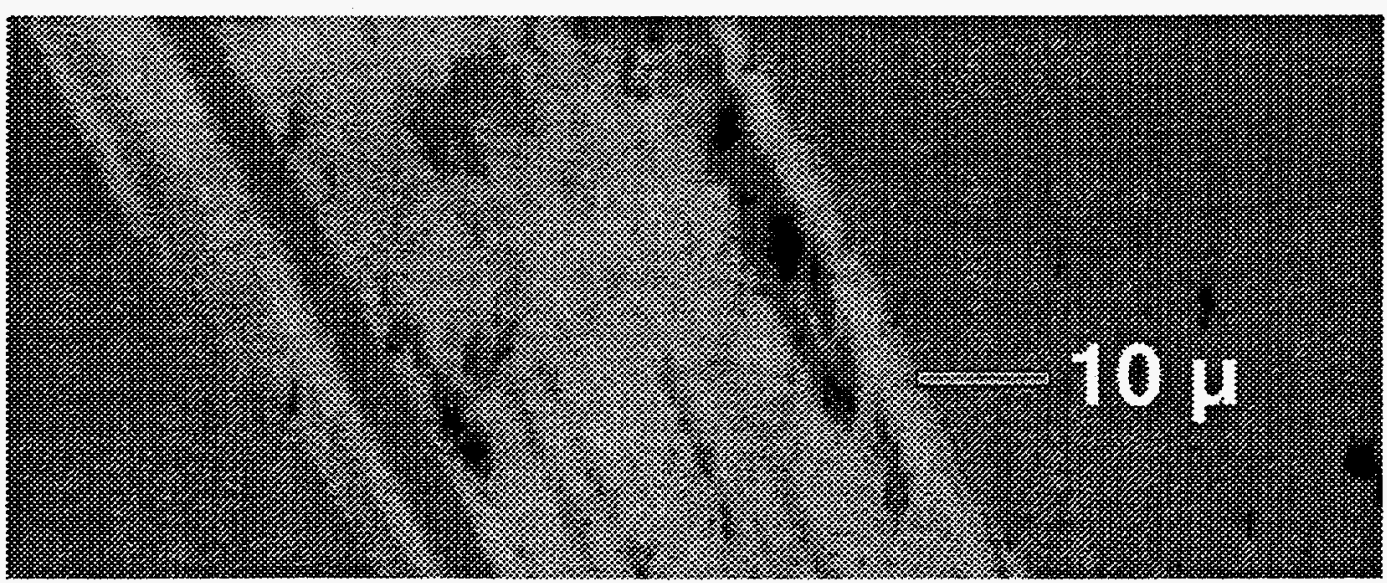

(c)

Figure 5.7. SEMs of the wear tracks after 660 cycles

(a) Unimplanted silicon (b) $4 \times 10^{17} \mathrm{~N}^{+}$ions $/ \mathrm{cm}^{2}$

(c) $8 \times 10^{17} \mathrm{~N}^{+}$ions $/ \mathrm{cm}^{2}$. 
Table 5.2 Roughness values of the implanted and unimplanted $\mathrm{Si}(100)$ samples after wear tests.

\begin{tabular}{|l|c|c|}
\hline \multicolumn{1}{|c|}{ Sample } & $\begin{array}{c}\text { RMS roughness of } \\
\text { the } 150 \text { cycles wear } \\
\text { track in } \mathrm{nm} \text { for a scan area } \\
2.5 \mu \mathrm{m} \times 2.5 \mu \mathrm{m}\end{array}$ & $\begin{array}{c}\text { RMS roughness of the } \\
660 \text { cycles wear track in } \mathrm{nm} \\
\text { for a scan area } \\
2.5 \mu \mathrm{m} \times 2.5 \mu \mathrm{m}\end{array}$ \\
\hline Unimplanted & 20.6 & 62.0 \\
\hline $4 \times 10^{11} \mathrm{C}^{+}$ions $/ \mathrm{cm}^{2}$ & 26.8 & 31.1 \\
\hline $4 \times 10^{11} \mathrm{~N}^{+}$ions $/ \mathrm{cm}^{2}$ & 2.0 & 49.0 \\
\hline $4 \times 10^{17} \mathrm{C}^{+}$ions $/ \mathrm{cm}^{2}+4 \times 10^{17} \mathrm{~N}^{+}$ions $/ \mathrm{cm}^{2}$ & 11.2 & 21.0 \\
\hline $8 \times 10^{1+} \mathrm{N}^{+}$ions $/ \mathrm{cm}^{2}$ & 79.7 & 44.5 \\
\hline $8 \times 10^{17} \mathrm{C}^{+}$ions $/ \mathrm{cm}^{2}$ & 8.8 & 18.1 \\
\hline
\end{tabular}


Wear track profiles were measured by profilometry for various samples, and the results are shown in Figures 5.8 and 5.9. The depth of the wear track was less than the depth of the modified region. The wear area was determined from these wear profiles. The wear factor, defined as the wear volume per unit load per unit sliding distance from Eqn.[3.14], after 150 and 660 cycles for all the implanted samples as well as the unimplanted sample, are shown in Figures 5.10 and 5.11, respectively. These data show that the trends in the wear factor determined after 150 cycles and 660 cycles were similar. The data also indicated that the carbon-implanted samples had a lower wear factor as compared to the nitrogen-implanted and unimplanted samples.

Figure 5.12 shows the wear factor as a function of hardness. These data showed that samples with high hardness had a low wear factor, and samples with low hardness had a high wear factor. Thus, carbon-implanted samples with high hardness had a lower wear factor than the nitrogen-implanted and unimplanted samples. 


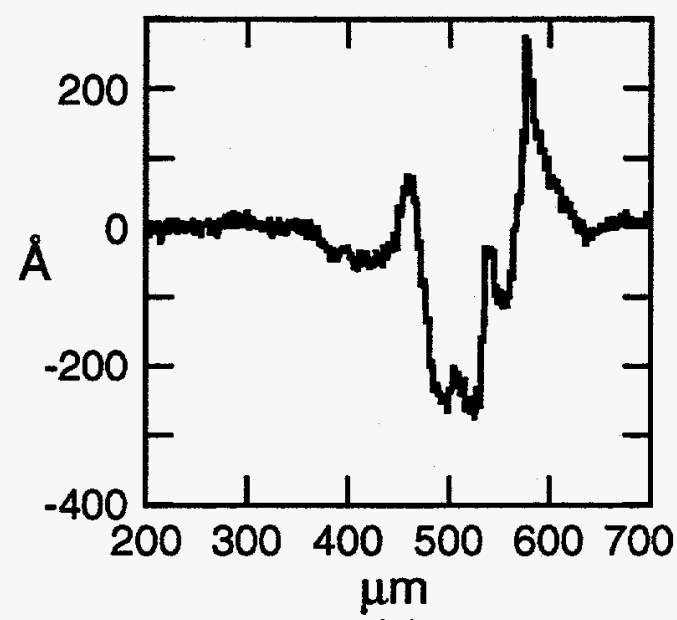

(a)

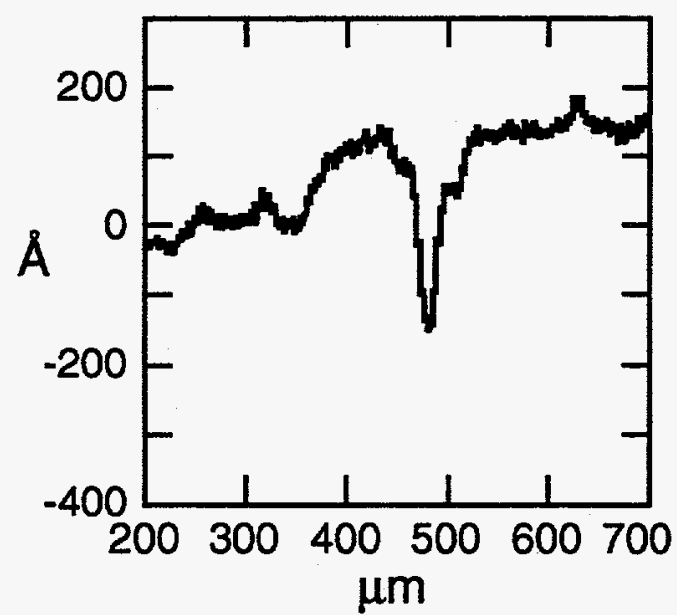

(c)

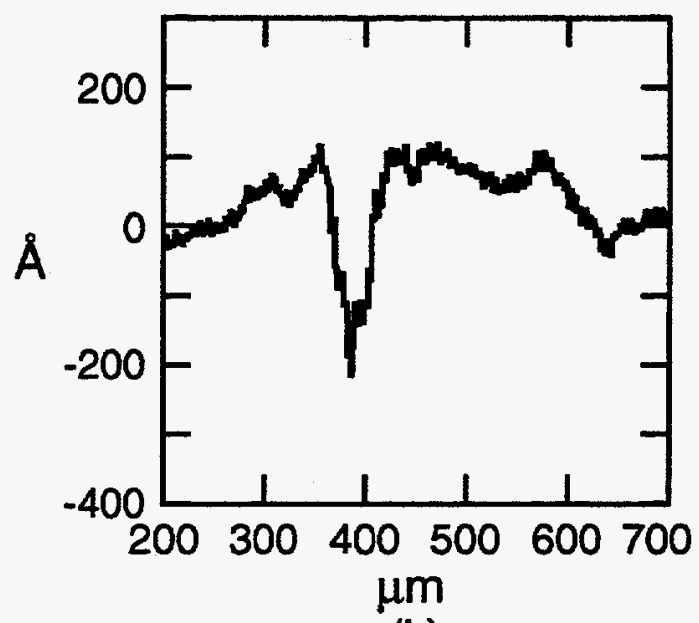

(b)

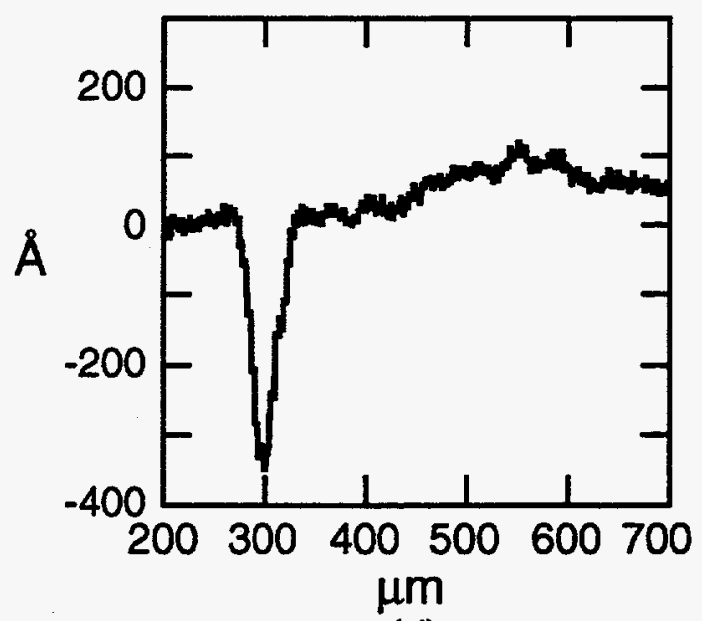

(d)

Figure 5.8. Wear track profiles for $\mathrm{Si}(100)$ after 150 cycles

(a) Unimplanted silicon

(b) $4 \times 10^{17} \mathrm{C}^{+}$ions $/ \mathrm{cm}^{2}$

(c) $4 \times 10^{17} \mathrm{C}^{+}$ions $/ \mathrm{cm}^{2}+4 \times 10^{17} \mathrm{~N}^{+}$ions $/ \mathrm{cm}^{2}$

(d) $4 \times 10^{17} \mathrm{~N}^{+}$ions $/ \mathrm{cm}^{2}$. 


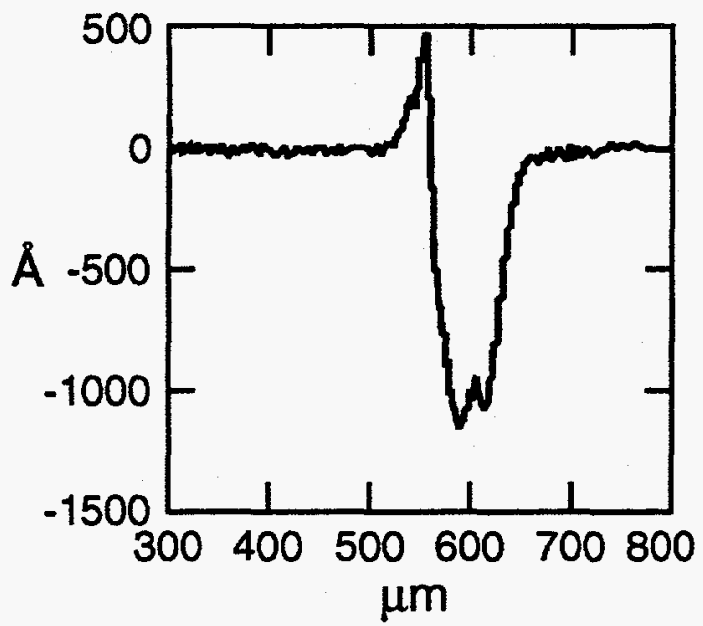

(a)

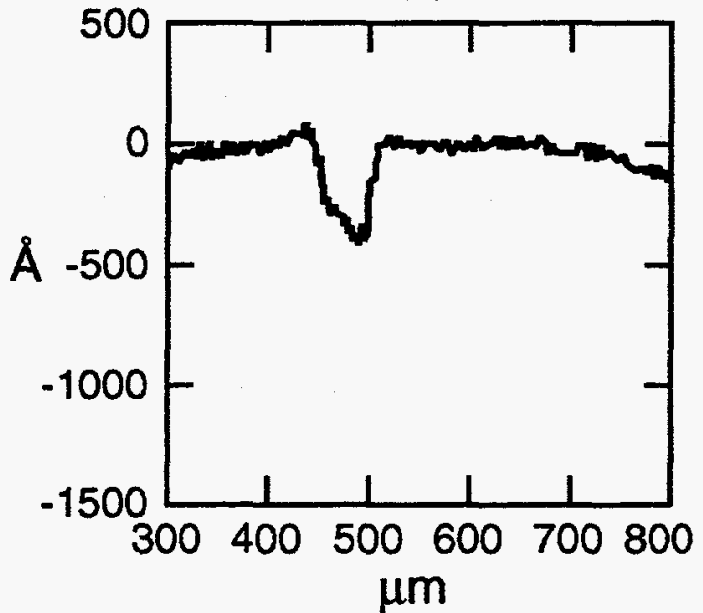

(c)

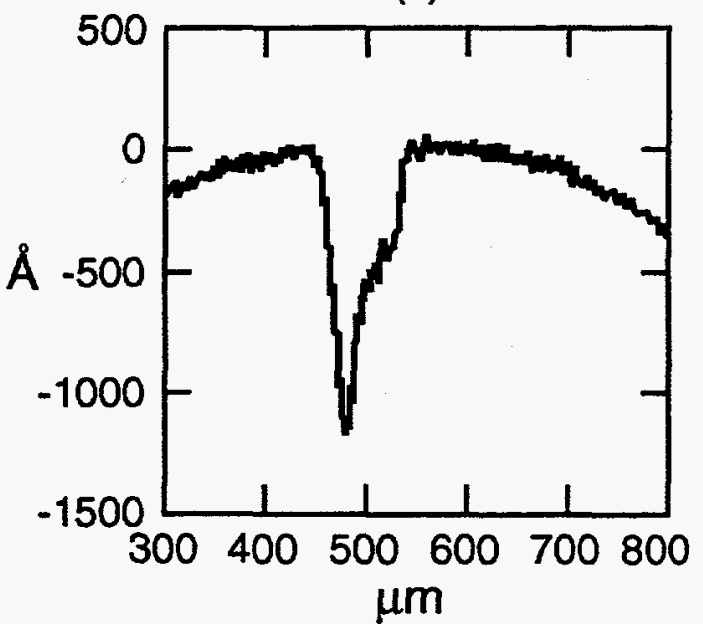

(e)

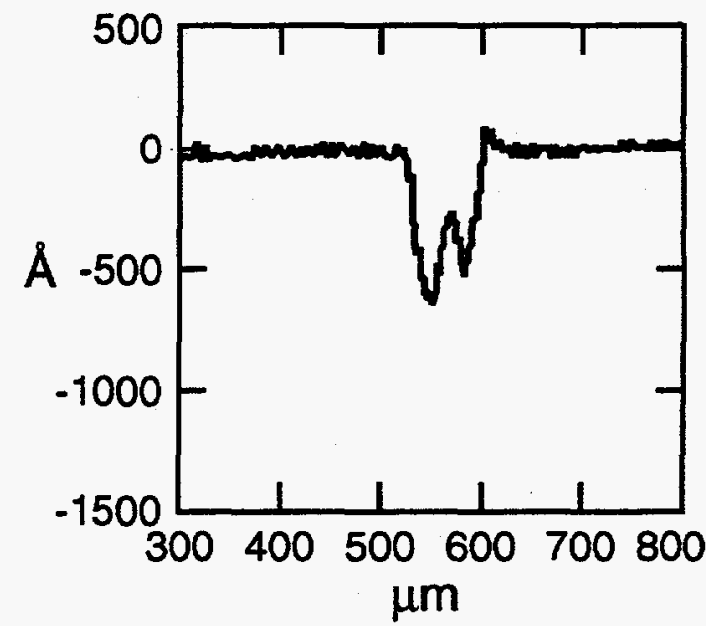

(b)

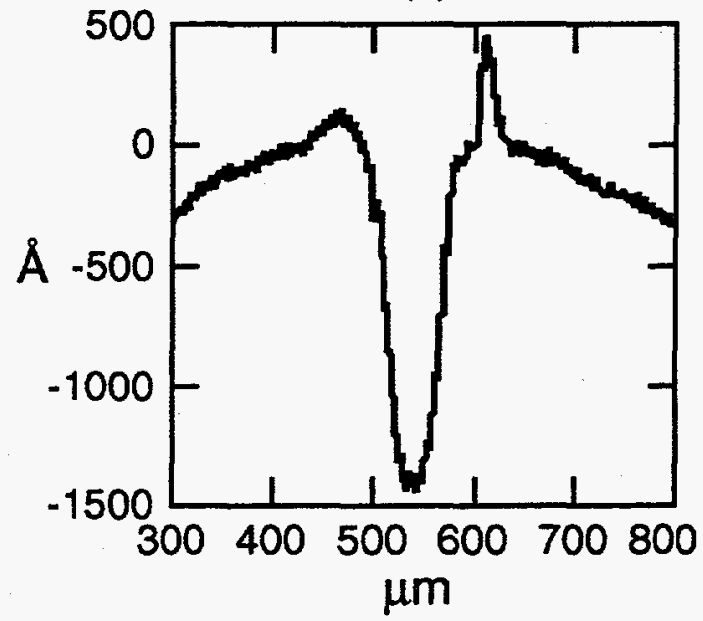

(d)

Figure 5.9. Wear track profiles for $\mathrm{Si}(100)$ after 660 cycles,
(a) Unimplanted silicon, (b) $4 \times 10^{17} \mathrm{C}^{+}$ions $/ \mathrm{cm}^{2}$,
(c) $8 \times 10^{17} \mathrm{C}^{+}$ions $/ \mathrm{cm}^{2}$ (d) $4 \times 10^{17} \mathrm{~N}^{+}$ions $/ \mathrm{cm}^{2}$,
(e) $4 \times 10^{17} \mathrm{C}^{+}$ions $/ \mathrm{cm}^{2}+4 \times 10^{17} \mathrm{~N}^{+}$ions $/ \mathrm{cm}^{2}$. 


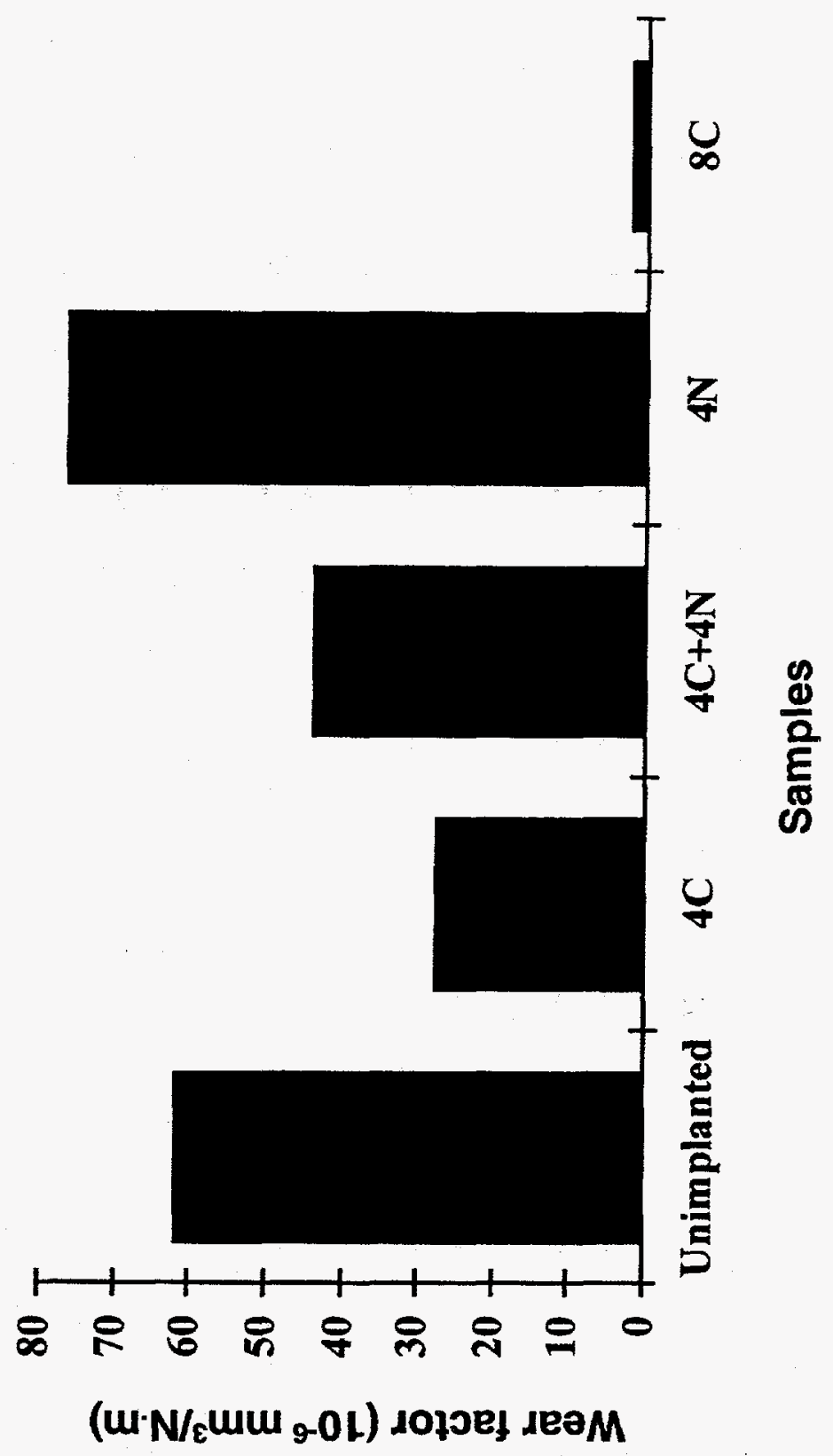

Figure 5.10 Wear factor as a function of different species and doses for 150 cycles. 


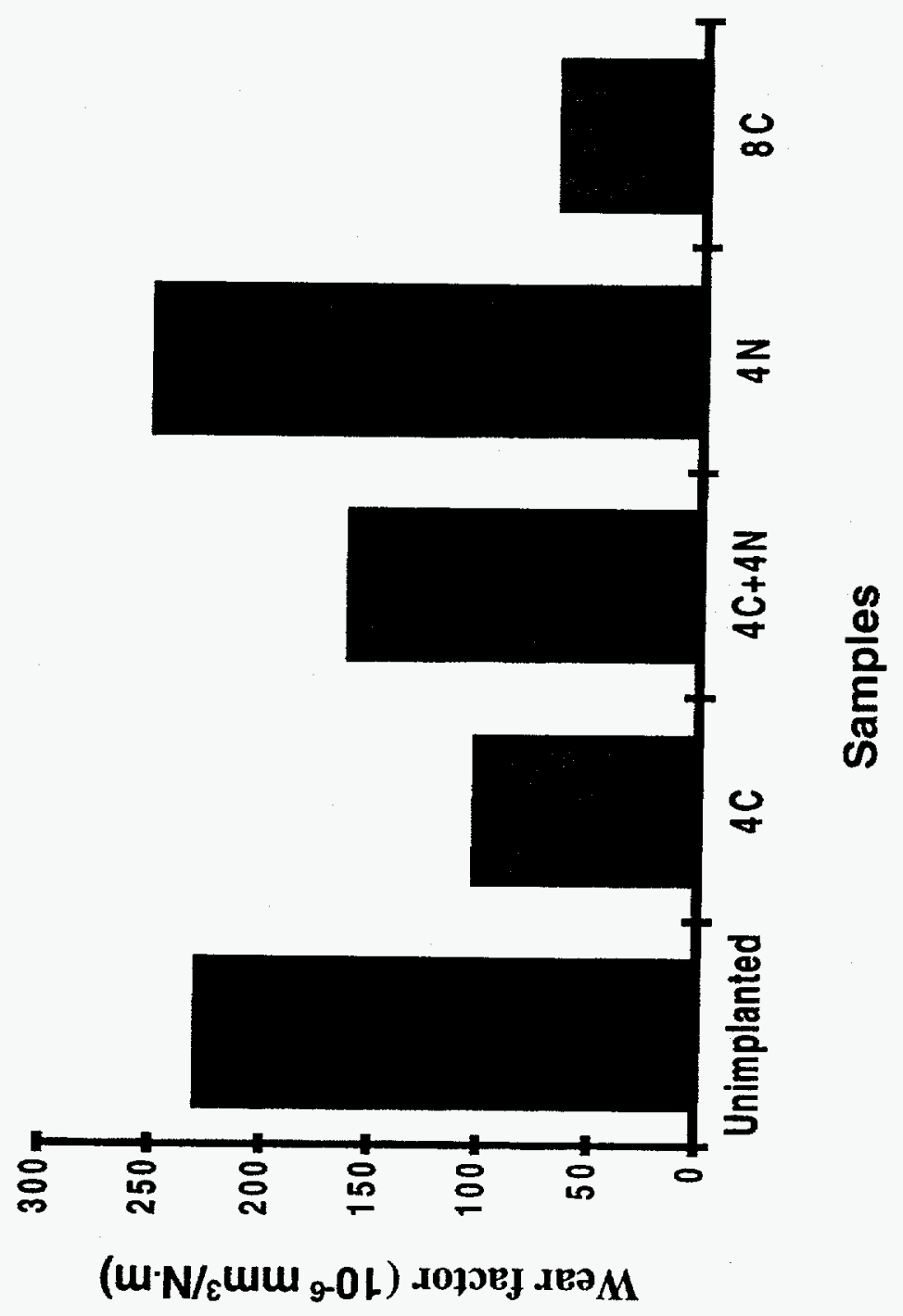

Figure 5.11 Wear factor as a function of different species and doses for 660 cycles. 


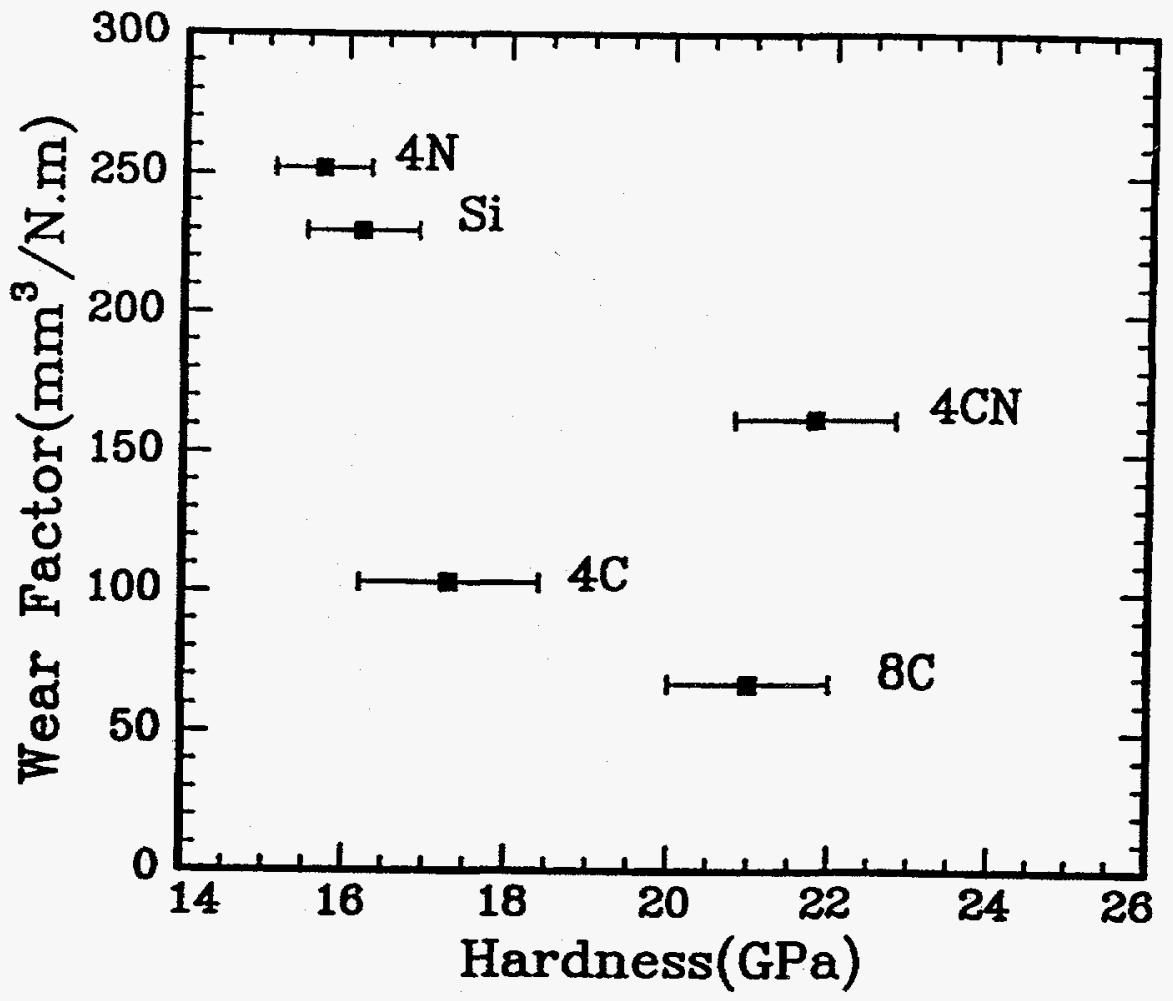

Figure 5.12 Wear factor for 660 cycles as a function of hardness. 


\subsection{Discussion of Results}

The following discussion addresses surface hardness, friction, and wear behavior of the unimplanted and implanted samples.

\subsubsection{Surface Hardness}

Hardness of a material is a measure of resistance to deformation. To a first order approximation, hardness of a material is related to its bond strength. Thus, the results of surface hardness of the implanted surfaces was explained using bond strength data. Since the implanted region had two different atoms ( $\mathrm{Si}$ and the implanted species), it is important to know the location and the diffusion of the implanted species in the silicon lattice. In addition, the solubility of the implanted species in a silicon lattice can be examined by applying Hume-Rothery rules [131]. In order to understand the possible sites of the implanted species, it is appropriate to examine the silicon structure.

Figure 5.13 shows the structure of silicon. There are 8 atoms and 8 interstitials per unit cell. Taking the silicon atomic radius to be $0.146 \mathrm{~nm}$, only $34 \%$ of the volume of the unit cell is occupied and $66 \%$ of the unit cell is free volume. The atomic radius of the carbon and nitrogen ions are $0.091 \mathrm{~nm}$ and $0.075 \mathrm{~nm}$, respectively. The size of the interstitial site is $0.104 \mathrm{~nm}$. Thus, the relatively open structure of silicon suggests that carbon and nitrogen ions can easily fit into the silicon lattice as interstiatial impurities. size, electronegativity, and valency between solute and the solvent. The difference in atomic size must be no more than $15 \%$. The host and the impurity must have the same 


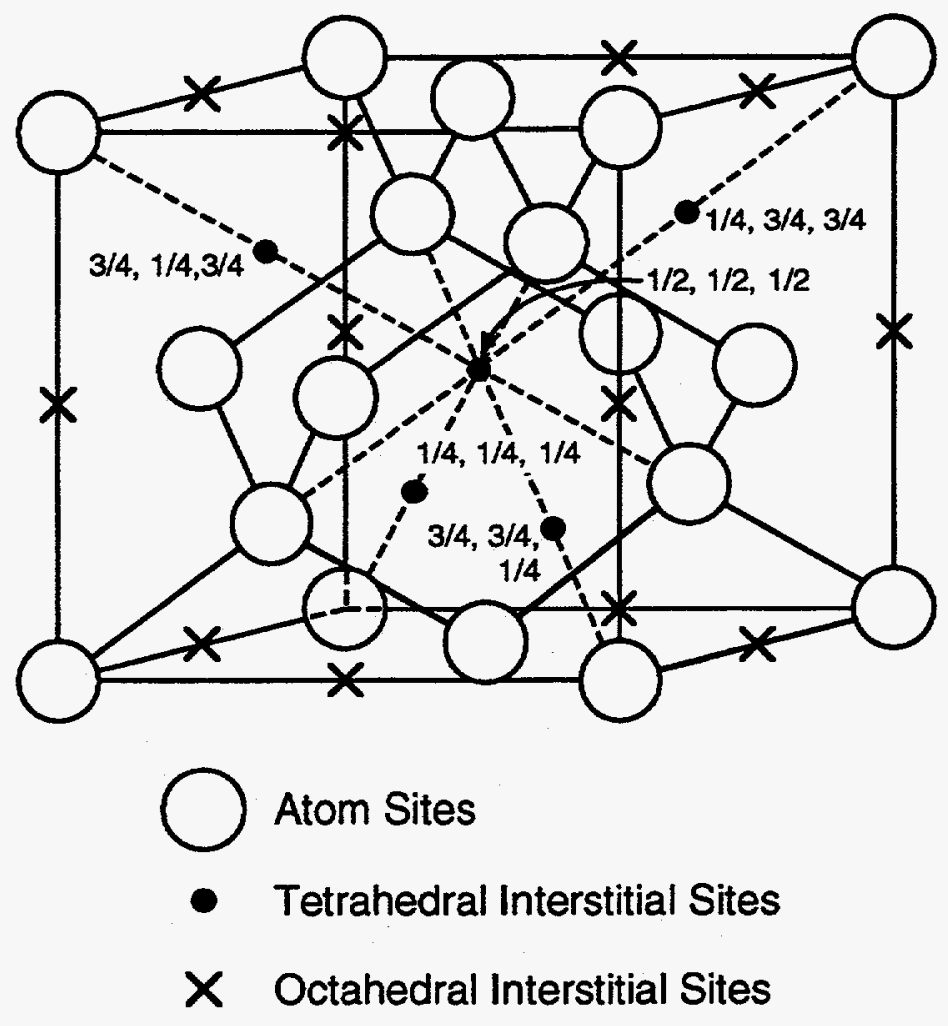

Figure 5.13. Structure of silicon. 
The solubility of carbon or nitrogen as a substitutional atom in a silicon lattice can be examined by applying Hume Rothery rules [131]. According to these rules, the solubility of the impurity (solute) is limited by the relative difference between atomic valence and about the same the electronegativity. These conditions are only necessary, but not sufficient to form complete solubility. It has been proposed that the electrochemical and relative valence effects dominate the magnitude of the solubility of impurities in silicon [132].

Table 5.3 summarizes the atomic radii, covalent radii, electronegativity, and the valency of silicon, carbon, and nitrogen atoms. Both carbon and nitrogen ions differ by more than $15 \%$ in size from the silicon ion, suggesting a limited solid solubility by substitution. The elements that have valence electrons in the $s$ and $p$ states may fit in with $\mathrm{sp}^{3}$ bonding of silicon either of by electron sharing or hybridizing. Because the carbon has 4 valency electrons, the formation of bonds between carbon and silicon is easy and strong. In contrast, nitrogen has 5 valency electrons, and thus will be electrically active in the silicon lattice. From the data in Table 5.3, and according to Hume Rothery rules [131], it is seen that the carbon and nitrogen cannot form a substitutional solid solution with silicon. Nitrogen and carbon ions are small and can be incorporated into the silicon lattice at interstitial sites to form a solid solution. Because nitrogen has five valency electrons, formation of an interstitial solid solution requires an associated charge balance to maintain electrical neutrality, which can be accomplished by vacancy formation.

In summary, both carbon and nitrogen have a tendency to form an interstitial solid solution in the silicon lattice. However, experimental studies involving the diffusivities of carbon in silicon have shown that the activation energy for diffusion and the diffusion coefficient are similar to those found for impurities which are known to diffuse substitutionally [134]. Thus, it appears likely that carbon enters as a substitutional impurity [134-135]. 
Ion implantation has been shown to be capable of forming metastable crystalline or amorphous solid solutions, with compositions exceeding the equilibrium solid solubility. Starting with pure silicon with 8 atoms per unit cell, 10.63 atoms of nitrogen or 8 of carbon are required to form a stoichiometric silicon nitride and silicon carbide. The maximum number of nitrogen or carbon ions that can fit in the free volume of a unit cell of silicon can be estimated by assuming that carbon or nitrogen ions can occupy all the unoccupied space in the silicon lattice. Under these assumptions, either 31.6 nitrogen ions or 17.5 carbon ions can occupy the empty space in a metastable silicon. This approximation gives the maximum upper limit of the number of interstitial atoms that can be placed in the silicon unit cell. Physically, one may propose that the nitrogen or carbon ions in the silicon structure might need to satisfy some optimum conditions for bonding and stability, suggesting that only a fraction of the 31.6 nitrogen ions or 17.5 carbon ions will occupy the empty space in a metastable silicon structure. In the formation of metastable Si-nitride, it is anticipated that the nitrogen concentration in solid solution can vary between 57 to 80 at.\%, whereas for $\mathrm{Si}$-carbide, the carbon concentration can vary between 50 to 69 at.\%. 


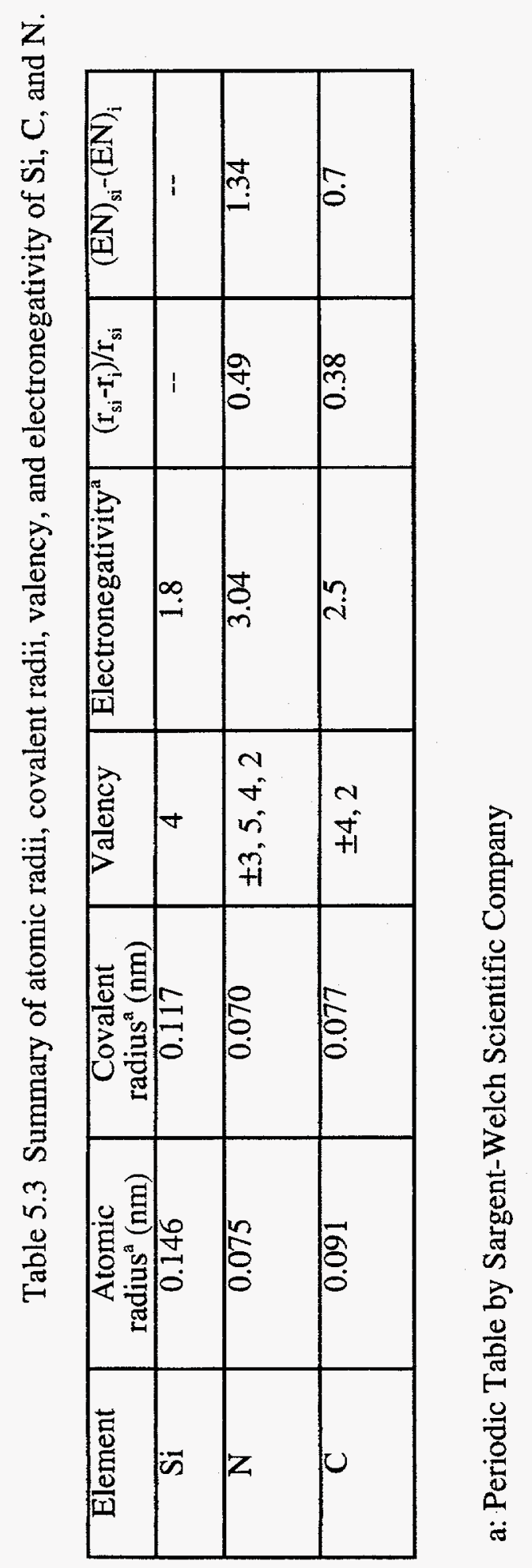


To understand the degree to which metastable solid solutions can form in $\mathrm{Si}$, the kinetics of the carbon and nitrogen into silicon were examined. The diffusion data of carbon and nitrogen in crystalline $\mathrm{Si}, \mathrm{SiC}$, and $\mathrm{Si}_{3} \mathrm{~N}_{4}$ are summarized in Table 5.4. TEM studies indicated that the carbon or nitrogen implanted silicon layer was amorphous.

Table 5.4 Summary of activation energy and diffusion constants of $\mathrm{C}$ and $\mathrm{N}$ into $\mathrm{Si}, \mathrm{SiC}$, and $\mathrm{Si}_{3} \mathrm{~N}_{4}$.

\begin{tabular}{|c|c|c|c|}
\hline $\begin{array}{c}\text { Diffusing } \\
\text { System }\end{array}$ & $\begin{array}{c}\text { Activation } \\
\text { energy } \\
Q(e V)\end{array}$ & $\begin{array}{c}\text { Diffusion constant } \\
\mathrm{D}_{0}\left(\mathrm{~cm}^{2} / \mathrm{sec}\right)\end{array}$ & Reference \\
\hline $\mathrm{C} \rightarrow \mathrm{Si}$ & 2.92 & 0.33 & {$[137,138]$} \\
\hline $\mathrm{N} \rightarrow \mathrm{Si}$ & 3.29 & 0.87 & {$[138]$} \\
\hline $\mathrm{C} \rightarrow \mathrm{SiC}$ & 5.42 & --- & {$[139]$} \\
\hline $\mathrm{N} \rightarrow \beta-\mathrm{Si}_{3} \mathrm{~N}_{4}$ & 8.1 & $5.8 \times 10^{6}$ & {$[140]$} \\
\hline $\mathrm{N} \rightarrow \mathrm{SiC}$ & --- & --- & -- \\
\hline
\end{tabular}

The following discussion is based on the assumption that the activation energies in amorphous $\mathrm{Si}, \mathrm{SiC}$, and $\mathrm{Si}_{3} \mathrm{~N}_{4}$ scale with crystalline materials. Cantor [139] argued that diffusion in amorphous alloys is faster than vacancy-controlled diffusion and slower than interstitial diffusion in crystalline materails. Using molecular dynamics simulations, Nordlund and Averback [139] have shown that the ion mixing in the amorphous structure of silicon is larger than in the crystalline structure. Since the activation energy for diffusion of carbon and nitrogen in $\mathrm{SiC}$ and $\mathrm{Si}_{3} \mathrm{~N}_{4}$ reported in the literature are high, diffusion via the vacancy mechanism is dominant in $\mathrm{C}$ and $\mathrm{N}$ implanted silicon. Comparing the activation energies in Table 5.4, one can conclude that the diffusion of carbon in the $\mathrm{SiC}$ lattice is easier than the diffusion of nitrogen in $\mathrm{Si}_{3} \mathrm{~N}_{4}$. 
Ion irradiation studies have indicated correlations between the atomic motion required to form equilibrium phases with the complexity and size of the unit cell [16]. Both $\alpha$ and $\beta$-silicon nitride have hexagonal structure with 14 and 28 atoms per unit cell, respectively, whereas, $\alpha$ and $\beta$-silicon carbide have 4 and 8 atoms per unit cell, respectively. This suggests that formation of silicon carbide is favored relative to silicon nitride by single implants of carbon and nitrogen ions into silicon.

The experimental data reported in section 5.2.1 show that nitrogen implantation into single-crystal silicon showed no improvement in surface hardness. For example, at a depth of $57 \mathrm{~nm}$ the hardness of the sample implanted with $4 \times 10^{17} \mathrm{~N}^{+}$ions $/ \mathrm{cm}^{2}$ was $15.7 \mathrm{GPa}$, which was almost equal to the hardness of the unimplanted sample, $16 \mathrm{GPa}$ (Figure 5.1).

Nitrogen has five valence electrons, when implanted into silicon, it has tendency to form an N-N bond rather than an Si-N bond. Infrared (IR) studies by Stein and Abe $[140,141]$ suggested that nitrogen atoms in silicon with concentrations below the equilibrium solid solution are in N-N pairs. Because the bond energy of Si$\mathrm{N}$ is greater than the Si-Si bond, one would expect increased hardness if the number of $\mathrm{Si}-\mathrm{N}$ bonds are significant. On other hand, if the $\mathrm{N}$ ions bond to other $\mathrm{N}$ ions, nitrogen molecules are formed which eventually can be trapped as nitrogen bubbles. Thus little or no hardening will be expected when N-N bonds are not significant. Surface hardness data indicated that there was no change in surface hardness, suggesting that the number of Si-Si bonds were unchanged in the sample implanted at a low dose.

When the dose was increased to $8 \times 10^{17} \mathrm{~N}^{+}$ions $/ \mathrm{cm}^{2}$, the measured hardness was $12.3 \mathrm{GPa}$ (Figure 5.1). This value of hardness was less than the value of the hardness of the unimplanted sample. RBS data that was discussed in Chapter 4 suggested that the peak atomic fraction of nitrogen at high dose was greater than 0.57 , the fraction that is required to form a stoichiometric equilibrium silicon nitride. TEM 
studies (Figure 4.6) revealed nitrogen bubbles in the entire modified region at this high dose, suggesting at this dose the nitrogen has exceeded the solid solubility limit in amorphous Si-N. Based on this RBS data (Table 4.3) and TEM data (Figures 4.7 and 4.8), one may conclude that the nitrogen solubility in amorphous $\mathrm{Si}-\mathrm{N}$ is above 0.47 and below 0.73 .

At a high dose, it is appropriate to think of nitrogen implantation in $\mathbf{S i}-\mathbf{N}$ material. As discussed earlier, when nitrogen is implanted into silicon, it more likely to form $\mathrm{N}-\mathrm{N}$ bonds rather than $\mathrm{Si}-\mathrm{N}$ bonds. In addition, the diffusion data also suggest that the diffusion of nitrogen in $\mathrm{Si}_{3} \mathrm{~N}_{4}$ is small. Thus, the number of $\mathrm{N}-\mathrm{N}$ bonds may increase with dose and the Si-Si bond density decreases. The $\mathrm{N}-\mathrm{N}$ bonds form nitrogen molecules. Since nitrogen is a gas at room temperature, when $\mathrm{N}-\mathrm{N}$ molecules reaches a critical number, nitrogen bubbles can form. This is consistent with observations made by others [142]. Thus, the decrease in hardness relative to the unimplanted sample appeared to be due to porosity of material due to the formation of bubbles and decrease in Si-Si bonds.

In summary, the present data suggests that silicon implanted with $4 \times 10^{17} \mathrm{~N}^{+}$ions $/ \mathrm{cm}^{2}$ is amorphous, and when the dose was increased to $8 \times 10^{17} \mathrm{~N}^{+}$ions $/ \mathrm{cm}^{2}$, surface softening was observed. This is consistent with observations made by Burnett and Page [24] that surface softening in silicon will result at a critical dose of $4 \times 10^{17} \mathrm{~N}^{+}$ions $/ \mathrm{cm}^{2}$.

In contrast, in the carbon-implanted samples, the surface hardness increased at both doses, i.e., $4 \times 10^{17} \mathrm{C}^{+}$ions $/ \mathrm{cm}^{2}$ and $8 \times 10^{17} \mathrm{C}^{+}$ions $/ \mathrm{cm}^{2}$, relative to the unimplanted sample (Figure 5.2). As mentioned earlier, formation of bonds between carbon and silicon is easy and strong due to its electronic configuration. Thus, when carbon is implanted into silicon, formation of Si-C bonds is expected. Kimura et al. [143] reported formation of disordered $\mathrm{Si}-\mathrm{C}$ bonds using IR absorption measurements in silicon implanted with carbon ions with an energies ranging from $40-70 \mathrm{keV}$. The 
bond energy of Si-C is $439 \mathrm{~kJ}$.mole $\mathrm{e}^{-1}$, which is $25 \%$ greater than Si-Si bond energy [146]. Thus, one expects an increase in hardness of carbon-implanted silicon.

Experimental measurements indeed show that the hardness of the sample increased by $6 \%$ at low dose of carbon. Gupta et al. [26] reported no change in the hardness of silicon was observed at doses below $2.5 \times 10^{17} \mathrm{C}^{+}$ions $/ \mathrm{cm}^{2}$ at $100 \mathrm{keV}$.

When the dose was increased from $4 \times 10^{17} \mathrm{C}^{+}$ions $/ \mathrm{cm}^{2}$ to $8 \times 10^{17} \mathrm{C}^{+}$ions $/ \mathrm{cm}^{2}$, it was expected that the number of Si-C bonds would increase. Although RBS data suggested that the peak atomic fraction of carbon was 0.72 , TEM data indicated no bubbles in the modified region. As explained in the Chapter 4, carbon ions can form bonds with other carbon ions and remain as solid at room temperature. TEM investigations by Kimura et al. [144] suggested that when the dose was greater than that corresponding to a stoichiometric concentration of carbon in a $\beta$-SiC, the excess carbon atoms remained as carbon clusters.

Consistent with the TEM observations by Kimura et al.[144], it is proposed that the carbon-implantation into silicon at high dose will produce $\mathrm{Si}-\mathrm{C}$ bonds and carbon clusters when the dose exceeds the critical limit for metastable Si-carbide. The bond energies of $\mathrm{C}-\mathrm{C}$ and $\mathrm{Si}-\mathrm{C}$ is greater than the $\mathrm{Si}-\mathrm{Si}$ bond energy. The resistance to plastic flow or the hardness directly scales with bonding energy, and thus the hardness in the carbon-implanted samples should increase with an increase in dose. The experimental measurements indicated a $30 \%$ increase in surface hardness at a high dose of carbon as compared to unimplanted sample. These results are also consistent with the work by Armstrong et al. [180]in the C-implanted Si(111).

In the case of the mixed implant sample $\left(4 \times 10^{17} \mathrm{C}^{+}\right.$ions $/ \mathrm{cm}^{2}$ and $4 \times 10^{17} \mathrm{~N}^{+}$ ions $/ \mathrm{cm}^{2}$ ), the surface hardness data showed a trend similar to the sample implanted with carbon at $8 \times 10^{17} \mathrm{C}^{+}$ions $/ \mathrm{cm}^{2}$ as shown in Figure 5.1. These data are in sharp contrast to samples implanted with only nitrogen ions. These data suggest that the 
presence of carbon ions in silicon modified the ability of the sample to effectively incorporate nitrogen ions into the Si-C implanted layer.

In the mixed implant $\left(4 \times 10^{17} \mathrm{C}^{+}\right.$ions $/ \mathrm{cm}^{2}$ to $4 \times 10^{17} \mathrm{~N}^{+}$ions $\left./ \mathrm{cm}^{2}\right)$, it is speculated that the nitrogen implantation into the carbon-implanted silicon displaced the carbon atoms and formed silicon nitride. The heats of formation for $\mathrm{SiC}$ and $\mathrm{Si}_{3} \mathrm{~N}_{4}$ are $-16.0 \mathrm{kcal} . \mathrm{mole}^{-1}$ and $-178.0 \mathrm{kcal} . \mathrm{mole}^{-1}$, respectively [145]. Nitrogen implantation into carbon-implanted silicon can be written in the form of chemical equation, as $3 \mathrm{SiC}+2 \mathrm{~N}_{2} \rightarrow \mathrm{Si}_{3} \mathrm{~N}_{4}+3 \mathrm{C}-130$ kcal.mole ${ }^{-1}$

This equation states that the reaction to form a silicon nitride from a mixture of nitrogen and $\mathrm{SiC}$ is exothermic, and thus formation of silicon nitride is thermodynamically favorable when nitrogen is implanted into carbon-implanted silicon. Similar arguments were made by Nastasi et al. [120] to explain the precipitation of carbon or boron nitride when nitrogen was implanted into polycrystalline $\mathrm{SiC}$ and $\mathrm{B}_{4} \mathrm{C}$, respectively. The bonds that result due to a mixed implantation are Si-N, Si-C, and C-C. Because the bond energies of $\mathrm{Si}-\mathrm{N}$ and $\mathrm{Si}-\mathrm{C}$ are greater than $\mathrm{Si}-\mathrm{Si}$, an increase in the hardness was expected. The experimental measurements indicated an increase in surface hardness of the mixed implant sample.

\subsubsection{Friction and Wear Behavior}

The sample implanted with $4 \times 10^{17} \mathrm{~N}^{+}$ions $/ \mathrm{cm}^{2}$ showed a slight decrease in the coefficient of friction relative to the unimplanted sample, whereas the sample implanted with $8 \times 10^{17} \mathrm{~N}^{+}$ions $/ \mathrm{cm}^{2}$ showed an increase in the coefficient of friction. For the sample implanted with $8 \times 10^{17} \mathrm{~N}^{+}$ions $/ \mathrm{cm}^{2}$, the observed bubbles from TEM studies and higher roughness from AFM studies may have contributed to the increase in the friction. The surface softening of this sample could have increased the ploughing and thus raised the friction.

For the carbon-implanted samples $\left(4 \times 10^{17} \mathrm{C}^{+}\right.$ions $/ \mathrm{cm}^{2}$ and $8 \times 10^{17} \mathrm{C}^{+}$ions $\left./ \mathrm{cm}^{2}\right)$, a low friction for about 300 cycles was observed. After 300 cycles, friction changed 
to the value of the unimplanted sample (0.6). However, in the mixed-implanted sample $\left(4 \times 10^{17} \mathrm{C}^{+}\right.$ions $/ \mathrm{cm}^{2}+4 \times 10^{17} \mathrm{~N}^{+}$ions $\left./ \mathrm{cm}^{2}\right)$, a low coefficient of friction was observed for about 100 cycles. This observation suggested that the carbon was responsible for the low coefficient of friction. The transition of low friction to high friction occurred in the carbon-implanted samples before the modified layer had worn off completely. For example, for the sample implanted with $4 \times 10^{17} \mathrm{C}^{+}$ions $/ \mathrm{cm}^{2}$, the depth of the wear track measured by profilmetry after 660 cycles (Figure $5.9 a$ ) was 70 $\mathrm{nm}$, and was less than the width of the modified layer, $270 \mathrm{~nm}$. It is speculated that the graphitization at the outermost surface layer was responsible for the lower friction coefficient in the carbon implanted samples.

Roughness measurements from AFM studies indicated that for the same sample $\left(4 \times 10^{17} \mathrm{C}^{+}\right.$ions $\left./ \mathrm{cm}^{2}\right)$, the roughness of the wear track after 150 cycles was 26.8 $\mathrm{nm}$, which was 100 times higher than the roughness before the wear tests (Tables 4.7 and 5.2). Loose wear debris in the wear tracks of the samples after 150 cycles (Figure 5.5) suggested that the wear particles that formed during the test might have been responsible for an increased friction coefficient before the modified layer had worn off.

The parallel grooves running along the wear track suggested an abrasive wear mechanism. As mentioned earlier, loose wear debris seen inside the track suggested third-body wear. It is hard to differentiate after the fact between abrasive and adhesive wear. In this study, the density of parallel grooves running along the wear track were taken as a measure of severity of abrasive wear. Abrasive wear was more dominant in the sample treated with a dose of $8 \times 10^{17} \mathrm{~N}^{+}$ions $/ \mathrm{cm}^{2}$, whereas adhesive wear was more dominant in carbon-implanted samples. In the unimplanted sample, both abrasive and adhesive wear mechanisms were present. However, in the case of samples implanted with carbon, the number of parallel grooves was less, suggesting that the abrasive wear mechanism was less dominant. 
Trends observed in the wear factor after 150 and 660 cycles were similar. A reduction in wear factor was observed at 150 cycles for all the implanted samples, with the exception of $4 \times 10^{17} \mathrm{~N}^{+}$ions $/ \mathrm{cm}^{2}$. There was large scatter in the data of the wear factor of the sample implanted with $8 \times 10^{17} \mathrm{~N}^{+}$ions $/ \mathrm{cm}^{2}$. The nitrogen bubbles indicated by TEM studies in this sample (Figure 4.6) could be responsible for the scattered wear data.

At 150 cycles, the carbon-implanted samples showed a reduction in the wear factor relative to the unimplanted sample. The lowest wear factor was exhibited by the sample with high hardness, which was $8 \times 10^{17} \mathrm{C}^{+}$ions $/ \mathrm{cm}^{2}$ (Figure 5.10). The wear factor for the mixed sample $\left(4 \times 10^{17} \mathrm{C}^{+}\right.$ions $/ \mathrm{cm}^{2}+4 \times 10^{17} \mathrm{~N}^{+}$ions $\left./ \mathrm{cm}^{2}\right)$ was less than the wear factor for the sample implanted with $4 \times 10^{17} \mathrm{~N}^{+}$ions $/ \mathrm{cm}^{2}$ and greater than the sample treated to a dose of $4 \times 10^{17} \mathrm{C}^{+}$ions $/ \mathrm{cm}^{2}$. This result suggested that carbon had the greatest influence on improving wear properties of the mixed-implant sample.

The wear factor of the carbon-implanted samples after 660 cycles was reduced significantly as compared to the unimplanted sample (Figure 5.11). For example, the wear factor for the sample implanted with $8 \times 10^{17} \mathrm{C}^{+}$ions $/ \mathrm{cm}^{2}$ was reduced by $70 \%$ relative to the unimplanted sample. As observed at 150 cycles, the wear factor for the mixed-implant sample after 660 cycles was less than the wear factor for the sample implanted with $4 \times 10^{17} \mathrm{~N}^{+}$ions $/ \mathrm{cm}^{2}$ and greater than the sample treated to a dose of $4 \times 10^{17} \mathrm{C}^{+}$ions $/ \mathrm{cm}^{2}$. Although the hardness of the mixed-implant sample $(4 \mathrm{C}+4 \mathrm{~N})$ and the sample implanted with $8 \times 10^{17} \mathrm{C}^{+}$ions $/ \mathrm{cm}^{2}$ was same, the wear factors of these samples after 660 cycles differed by a factor 2.4 . The trends observed in the wear factor after 660 cycles were similar to those observed at 150 cycles. The response of carbon- and nitrogen-implanted silicon with the 52100 pin might be different, leading to unequal wear factors. The data also suggested that implantation of nitrogen into silicon degrades the improved wear behavior expected from carbon implantation. A significant reduction in the wear factor compared to the unimplanted sample was 
observed for carbon-implanted samples after 660 cycles (Figure 5.11). For example, the wear factor for the sample implanted with $8 \times 10^{17} \mathrm{C}^{+}$ions $/ \mathrm{cm}^{2}$ was reduced by $70 \%$ relative to the unimplanted sample.

The plasticity index values can be estimated using Eqn. [2.1] and from RMS roughness values obtained from AFM measurements. The values of the plasticity index between the pin and the disc before the friction and wear test were less than 0.6. This suggested that the contact between them was elastic for all the samples [58]. The calculated values of $\Psi$, the plasticity index after the wear test for 150 cycles, was $<0.6$ for all samples except for the sample treated to a dose of $8 \times 10^{17} \mathrm{~N}^{+}$ions $/ \mathrm{cm}^{2}$. The sample implanted with $8 \times 10^{17} \mathrm{~N}^{+}$ions $/ \mathrm{cm}^{2}$ had a plasticity index of 0.75 after a wear test for 150 cycles, indicating the deformation was elasto-plastic at a lower number of cycles. The plasticity index value calculated after 150 cycles for other samples was less than 0.6 ; however, the wear morphology indicated a plastic deformation. This discrepancy in the predicted and observed nature of the deformation at a lower number of cycles may have been due to the following reasons. The plasticity index expression was derived for static contact, whereas the experimental investigation corresponded to repeated sliding of the pin on the disc. As the test progressed, the surfaces and contact radii can change due to wear. Also the assumption that pin roughness was to be equal that of the wear track.

The plasticity index value for carbon-implanted samples and the sample treated to a dose of $4 \times 10^{17} \mathrm{~N}^{+}$ions $/ \mathrm{cm}^{2}$ was greater than 0.6 , but less than unity as the test continued for higher number cycles, i.e., 660 cycles. The nature of the deformation changed from elastic to elasto-plastic for these samples. In the case of the unimplanted sample and the sample implanted with $8 \times 10^{17} \mathrm{~N}^{+}$ions $/ \mathrm{cm}^{2}$, the plasticity index value exceeded 1.0 , indicating that the deformation behavior was predominantly plastic. Thus, the severity of the abrasive wear scaled with the amount of plastic deformation and can be visualized by the plasticity index value. 
Examination of the trends in the wear factor and hardness data revealed an inverse correlation between hardness and wear properties, as in Archard's law, Eqn. [2.4]. For example, samples with high hardness had a low wear factor, and samples with low hardness had a high wear factor. Interestingly, samples with low friction had a low wear factor, and samples with high friction had a high wear factor, suggesting a general correlation between friction and wear in this material system.

When the transition of low friction to high friction occurs, the location of maximum von Mises stress changes from the subsurface to the surface (Figure 2.7). Plastic deformation is one mechanism by which loss of material can occur during a sliding contact. The von Mises stress is a measure of the severity of plastic deformation; samples with high friction can lead to a larger wear factor as compared to the sample with low friction. Thus, the samples with high friction had a high wear factor.

Eqn. [2.16] implies that the tensile stress at the trailing edge scales with the friction coefficient. Thus, when the friction coefficient changes from 0.2 to 0.7 , the tensile stress at the trailing edge changes. The tensile stress at the trailing edge estimated from Eqn. [2.16] was $0.12 \mathrm{GPa}$ for the friction coefficient of 0.2 and 0.35 GPa for the friction coefficient of 0.7. Tensile stresses can lead to crack formation and eventually wear debris. Thus, high friction might have lead to more wear debris and thus a higher wear factor. These data suggested that there was a general correlation between friction and wear properties.

\subsection{Conclusions}

Surface hardness data suggested that when nitrogen was implanted into silicon, the tendency to form $\mathrm{N}-\mathrm{N}$ bonds was more dominant than $\mathrm{Si}-\mathrm{N}$ bonds. When the N-N bonds reached a critical density, nitrogen bubbles formed. The data also suggested that the maximum solid solubility of nitrogen in silicon was between 0.42 and 0.73 atomic percent. In contrast, carbon easily formed bonds with silicon because of its 
electronic configuration. In addition, carbon is a solid at room temperature, unlike nitrogen. Thus, the formation of $\mathrm{C}-\mathrm{C}$ bonds would not result in surface softening unless the entire modified region is graphite. Surface hardness was improved by implanting carbon into silicon. In the case of the mixed-implanted sample, where the nitrogen implantation was followed by carbon implantation, the incorporation of nitrogen into the silicon lattice was modified. As a result, surface hardness was improved significantly as compared to the unimplanted sample.

The wear factor of the sample implanted with a low dose of nitrogen was almost equal to the wear factor of the unimplanted sample, whereas high doses of nitrogen implantation degraded the wear behavior. Carbon-implantation improved the wear factor of silicon at both doses. The wear factor of carbon-implanted samples scaled inversely with hardness, as in Archard's law. The improved wear behavior of the samples implanted with both carbon and nitrogen was not as significant as the carbon-implanted samples. The value of the wear factor of this mixed sample was found to be the average value of the wear factors of the sample implanted at a low dose of nitrogen and a low dose of carbon. This suggests that both the carbon and nitrogen ions influenced the wear factor of the mixed implant sample. Mixed implantation of carbon and nitrogen did not result in any novel phases, with significantly different wear behavior than single implants of carbon. In summary, this chapter demonstrates, depending on the nature of the implant species, that ionimplantation can produce wear-resistant surfaces. Mixed implantation of carbon and nitrogen into silicon were investigated for the first time. 


\section{Chapter 6 Investigations of Amorphous Diamond-like Carbon Coatings}

The low friction and wear behavior of diamond-like carbon (DLC) coatings has significant impact on their potential applications. Some of these include increased performance and lifetime of cutting tools, gears, bearings, seals, hard disc drives, cams, etc. In addition, DLC coatings have excellent biocompatibility for use in biomedical applications [43].

Several researchers performed extensive studies on the tribological properties of amorphous DLC coatings processed by various techniques [43-53]. The percentage of $\mathrm{sp}^{3}$-bonds and the hydrogen content of the coatings dictate the hardness and elastic behavior. The coatings with a higher percentage of $\mathrm{sp}^{3}$-bonds and lower hydrogen content have higher hardness and modulus. In addition, residual stresses in these coatings has been shown to be correlated with hardness and $\mathrm{sp}^{3}$ content [52]. Investigations of tribological properties of amorphous DLC coatings with different hydrogen content revealed no major differences in frictional behavior, but indicated significant differences in wear rate [50].

Several processing techniques are available to synthesize DLC coatings. Processes now in development can be scaled to industrial production to be efficient and economical to produce strongly adherent conformal coatings. Plasma Source Ion Implantation (PSII), a conformal technique that was developed a decade ago, has been successfully used to modify the tribological properties of steel punches, injection molds, die castings, printed circuit board drills, dental tools, saw blades, etc. by implanting gaseous species such as carbon, nitrogen, and oxygen ions [32-40].

\subsection{Objective}

There are several reports on the characterization of mechanical and tribological properties of DLC coatings $[32,33,44-53]$. There are no reports on the wear behavior 
of these coatings at high contact stresses of the order of $1 \mathrm{GPa}$. At such high contact stress, one might expect that coatings may fail by brittle fracture, in which case, the wear process is controlled by fracture phenomena.

There are standardized testing methods for the fracture toughness of the homogenous materials. However, these tests can not be used to measure the fracture toughness of thin coatings or films beacuse of experimental difficulties associated with both extremely low loads and displacement measurements, and film damage during specimen preparation and/or load application. Coatings that are applied to improve either wear resistance or fatigue resistance are always attached to the substrates under service conditions. Therefore, it is legitimate to measure fracture toughness of a coating attached to a substrate. However, a clear understanding of substrate contribution, film thickness, and residual stresses due to processing must be established. In this study where the substrate is relatively brittle, it is useful to know how brittle the coating is and how the coating affects the friction of the coatingsubstrate system. Vickers microindentation was used to investigate the effective fracture toughness of a coating-substrate system as a function of residual compressive stresses caused by surface modification. In this case, the residual stress intensity factor comes from the superposition of stress intensity factors due to both the film and the substrate. Thus, the fracture toughness of coating-substrate systems determined by indentation technique is referred to as the apparent or effective fracture toughness of the coating-substrate system.

The aim of this research was to examine the tribological behavior of DLC coatings that were processed by the plasma source ion implantation (PSII) technique and to correlate this behavior with the other material properties. Thus, in addition to determining the apparent fracture toughness of DLC coatings, the coatings were also characterized for hydrogen content, residual stress, hardness, friction, and wear behavior. 


\subsection{Synthesis}

A pulse-biased method using the PSII setup that was discussed in Chapter 3 was used to deposit diamond-like carbon (DLC) coatings on silicon substrates. Before deposition, the substrates were sputter-cleaned with argon at 10 mTorr for 2 hours and at $2 \mathrm{kV}$ with 20 microsecond pulses to remove native oxides on the surface. Then the sputter-cleaned samples were implanted with carbon at 0.3 mTorr to create a functionally graded interface. Twenty microsecond pulses of $20 \mathrm{kV}$ bias were applied to the samples at 400 to $600 \mathrm{~Hz}$ in the implantation step. After implantation, the samples were sputter-cleaned again for 8 minutes at $10 \mathrm{mTorr}$ and $2 \mathrm{kV}$ to remove contamination from the implantation process. Depositions were done using 0.6 to 1.5 $\mathrm{kV}$ pulsed bias and $\mathrm{C}_{2} \mathrm{H}_{2}$ rf-plasma at 0.5 to 1.7 mTorr. The coating thickness was measured by using profilometry. The residual stresses introduced during the synthesis of these coatings were measured using a technique discussed in Chapter 3 and are summarized in Table 6.1.

\subsection{Results}

The evaluation of DLC coatings was based on composition, surface hardness, apparent fracture toughness, and tribological properties. The results are presented in the following order.

1) Composition Analysis

2.) Surface Hardness

3) Tribological Properties

4) Discussion of the Results.

\subsubsection{Composition Analysis}

Hydrogen content was analyzed using elastic recoil detection (ERD) spectrometry [118] as discussed in Chapter 3, Section 3.2.2. Figure 6.1 shows the ERD spectra for the sample $S 2$. The simulated spectra used to calculate the hydrogen content is also shown in the figure. ERD analysis revealed less than 30 at. \% 
hydrogen in all the DLC samples. The hydrogen and carbon contents in the coated samples are also summarized in Table 6.1. 


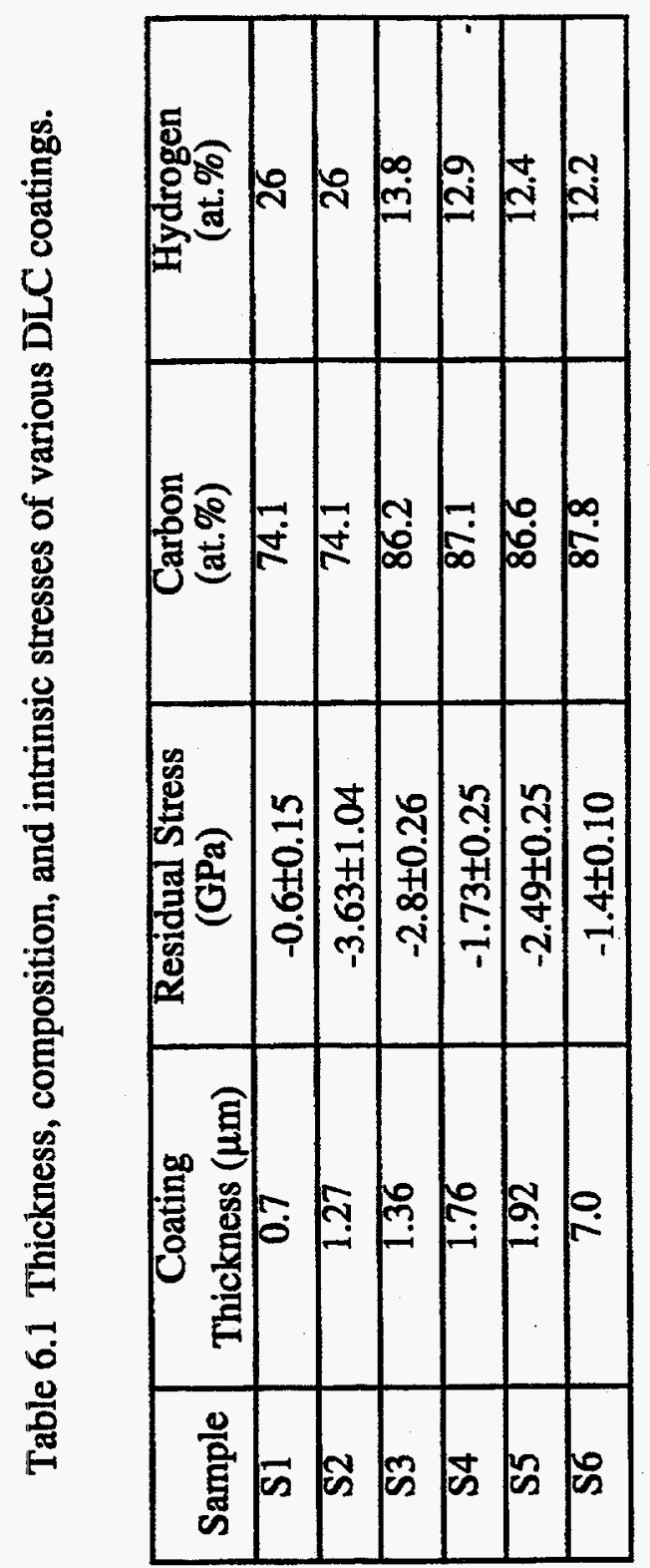




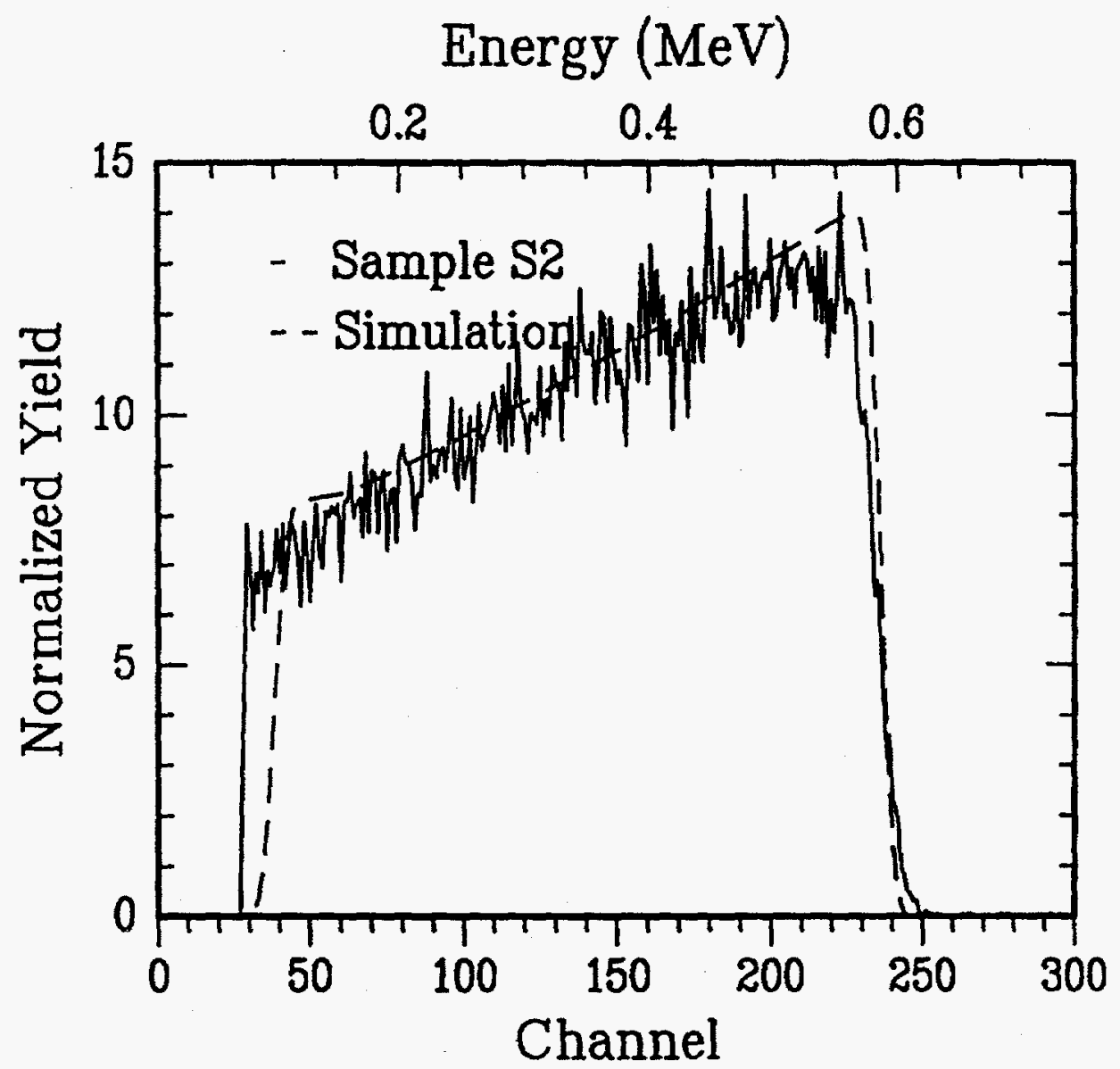

Figure 6.1 ERD spectra of sample S2. 


\subsubsection{Surface Hardness}

The hardness and modulus of the DLC coatings were determined using nanoindenter as discussed in Chapter 3, Section 3.2.4. Table 6.2 summarizes the results of hardness and the modulus values of the various coatings investigated. A comparison of the data from Table 6.1 and 6.2 show that the hardness is correlated with the film thickness. No other correlation exists. Figure 6.2 shows the variation of nanohardness measured at a penetration depth of $75 \mathrm{~nm}$ as a function of DLC coating thickness. When the thickness increased from 0.7 to $1.76 \mu \mathrm{m}$, a $70 \%$ increase in hardness occurred. This value remained constant up to $7 \mu \mathrm{m}$ thickness.

Table 6.2 Elastic moduli, hardness, and contact stresses of DLC coatings.

\begin{tabular}{|c|c|c|c|}
\hline Sample & $\begin{array}{c}\text { Coating } \\
\text { Thickness }(\mu \mathrm{m})\end{array}$ & $\begin{array}{c}\text { Elastic Modulus } \\
(\mathrm{GPa})\end{array}$ & $\begin{array}{c}\text { Hardness } \\
(\mathrm{GPa})\end{array}$ \\
\hline Uncoated & --- & 180 & 11.0 \\
\hline S1 & 0.7 & $113.6 \pm 4.7$ & $11.0 \pm 0.7$ \\
\hline S2 & 1.27 & $140.7 \pm 1.4$ & $16.7 \pm 0.2$ \\
\hline S3 & 1.36 & $145.8 \pm 2.6$ & $17.7 \pm 0.5$ \\
\hline S4 & 1.76 & $158.9 \pm 2.1$ & $18.95 \pm 0.4$ \\
\hline S5 & 1.92 & $159.9 \pm 11.5$ & $18.8 \pm 1.4$ \\
\hline S6 & 7.0 & $188.9 \pm 3.5$ & $19.0 \pm+0.7$ \\
\hline * Measurements taken at a depth of 75 nm \\
\hline
\end{tabular}

\subsubsection{Apparent Fracture Toughness}

A microindenter with loads ranging from 25 to 1000 grams was used to determine the cracking response of DLC coatings on silicon substrates. Figures 6.3a-b show the scanning electron micrographs of indents for bare silicon (6.3a) and Sample $S 4(6.3 b)$ at 1000 grams. The crack length was shorter in the coated sample (6.3b) 


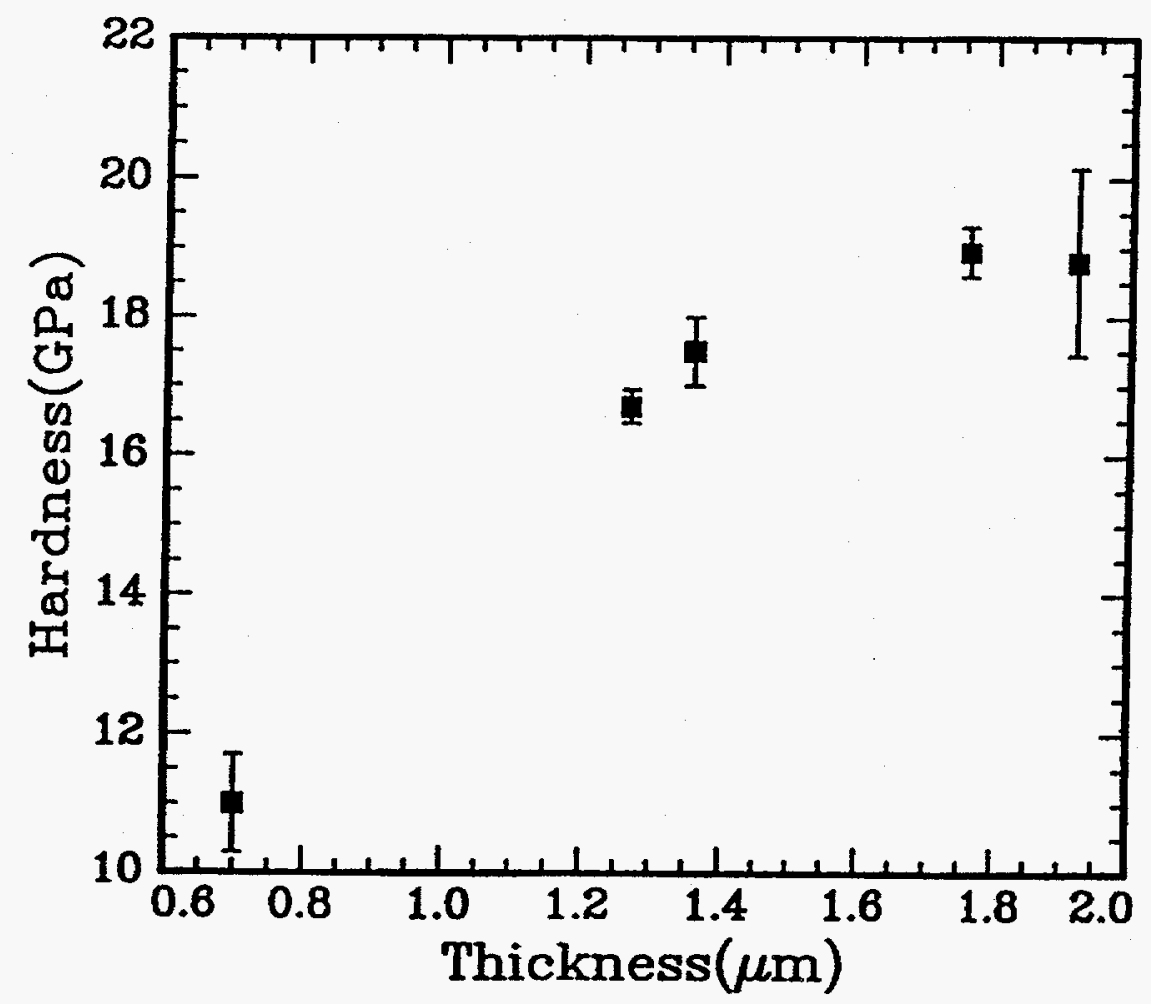

Figure 6.2 Dependence of hardness on coating thickness. 


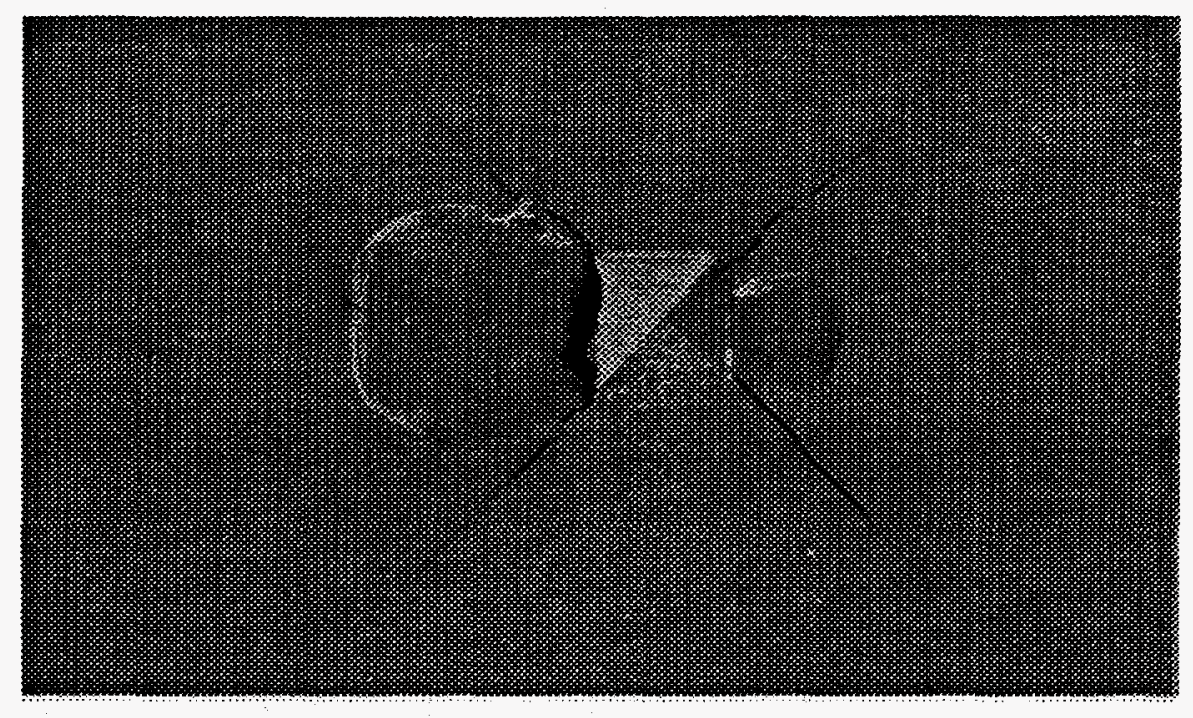

(a)

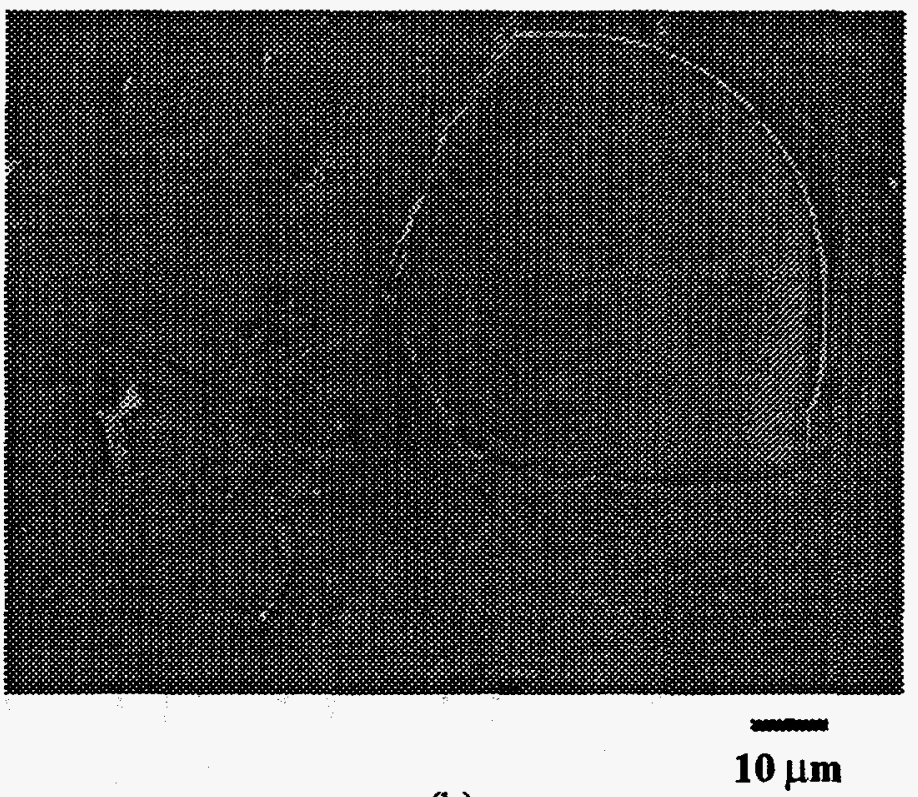

(b)

Figure 6.3 Scanning electron micrographs of the indents at 1000 grams (a) Uncoated silicon (b) Sample S4. 
compared to that of uncoated silicon (6.3a). Figure 6.4 shows the crack length dependence on load for the uncoated silicon and the sample S5. The length of the radial cracks generated at the corners of an indent were shorter in the coating compared to the uncoated silicon. Below 100 grams, no radial cracks were observed for DLC coatings in contrast to uncoated silicon. At 200 grams, the observed radial cracks did not meet the criteria, $\mathrm{C} \geq 2 \mathrm{a}$, where $\mathrm{C}$ is the crack length and $2 \mathrm{a}$ is the diagonal length of the indent. Thus, only well-defined cracks are included in Figure 6.4. The depth of an indent was greater than the coating thickness at all loads. The apparent fracture toughness calculated by Eqn. [3.15] is summarized in Table 6.3.

Table 6.3 Thickness, hardness, fracture toughness, and wear factor of DLC coatings.

\begin{tabular}{|c|c|c|c|}
\hline Sample & $\begin{array}{c}\text { Coating } \\
\text { Thickness }(\mu \mathrm{m})\end{array}$ & $\begin{array}{c}\text { Hardness } \\
(\mathrm{GPa})\end{array}$ & $\begin{array}{c}\overline{\mathrm{K}}_{\mathrm{r}} \\
\left(\mathrm{MPam}^{1 / 2}\right)\end{array}$ \\
\hline Virgin & ---- & 11.0 & $0.67 \pm 0.001$ \\
\hline S1 & 0.7 & $11.0 \pm 0.7$ & $0.77 \pm 0.002$ \\
\hline S2 & 1.27 & $16.7 \pm 0.2$ & $1.14 \pm 0.002$ \\
\hline S3 & 1.36 & $17.7 \pm 0.5$ & $1.31 \pm 0.003$ \\
\hline S4 & 1.76 & $18.95 \pm 0.4$ & $1.30 \pm 0.004$ \\
\hline S5 & 1.92 & $18.8 \pm 1.4$ & $1.52 \pm 0.002$ \\
\hline S6 & 7.0 & $19.0 \pm+0.7$ & -- \\
\hline
\end{tabular}

Trends in apparent fracture toughness with coating thickness and hardness are shown in Figures 6.5 and 6.6. Figure 6.5 shows that the apparent fracture toughness increased with DLC coating thickness, and Figure 6.6 shows that the apparent fracture toughness increased with DLC coating hardness. Interestingly, the $7 \mu \mathrm{m}$-thick sample S6 showed no radial cracks for loads as high as 3000 grams. Figure 6.7 shows the 


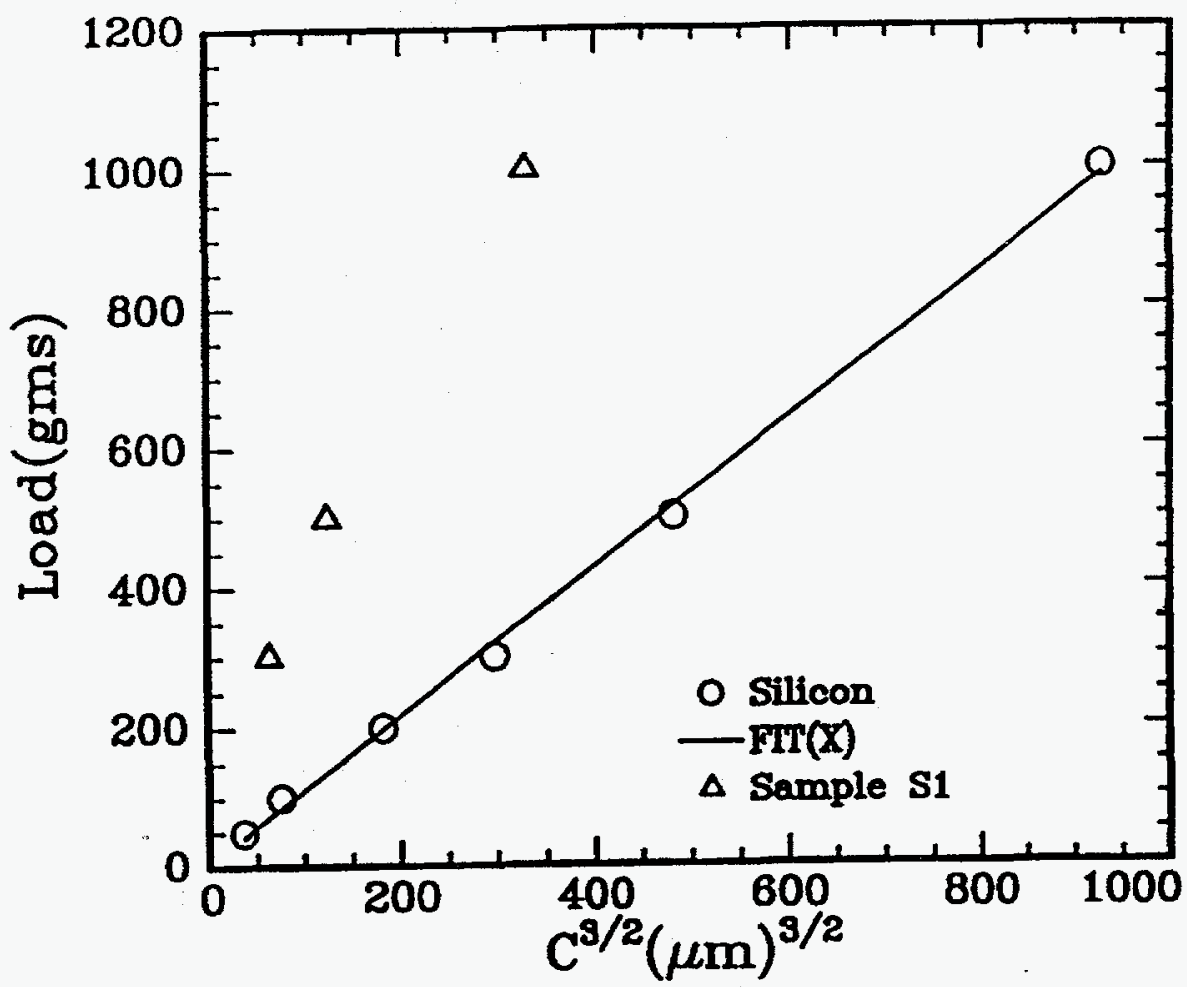

Figure 6.4 Results from the data of well-defined radial crack in uncoated silicon and DLC coating as a function of an applied load. 
SEM of the indent at 2000 grams for sample S6 showing no radial cracks. Thus, sample S6 was highly fracture resistant, with a low value of compressive stress.

\subsubsection{Tribological Studies}

Tribological studies were performed using a pin-on-disc (POD) apparatus under a normal load of $6.25 \mathrm{~N}$ and at $10 \%$ relative humidity, with a ruby pin as a slider. Static contact stress data for the coated and uncoated samples were calculated using Eqn.[2.6] and are summarized in Table 6.4. The DLC-coated substrates had different contact stresses than the uncoated silicon substrate because of the difference in elastic moduli. Figure 6.8 shows the coefficient of friction for the sample S4 and uncoated silicon at a load of 6.25 N. The coefficient of friction at an applied normal load of $6.25 \mathrm{~N}$ was observed to be less than 0.1 for all coated samples (S1-S6). Friction of the coated samples remained low even after 10,000 cycles, corresponding to a total sliding distance of 95 meters.

Table 6.4 Static contact stress, fracture toughness, and wear factor of DLC coatings.

\begin{tabular}{|c|c|c|c|}
\hline Sample & $\begin{array}{c}\text { Static Contact } \\
\text { Stress } \\
(\mathrm{GPa})\end{array}$ & $\begin{array}{c}\mathrm{K}_{\mathrm{r}} \\
\left(\mathrm{MPam}^{1 / 2}\right)\end{array}$ & $\begin{array}{c}\text { Wear Factor } \\
\left(\mathrm{mm}^{3} / \mathrm{N} . \mathrm{m}\right)\end{array}$ \\
\hline Virgin & 1.6 & $0.67 \pm 0.001$ & $3.17 \times 10^{-4}$ \\
\hline S1 & 1.25 & $0.77 \pm 0.002$ & $2.18 \times 10^{-7}$ \\
\hline S2 & 1.39 & $1.14 \pm 0.002$ & $1.65 \times 10^{-1}$ \\
\hline S3 & 1.412 & $1.31 \pm 0.003$ & $0.79 \times 10^{-1}$ \\
\hline S4 & 1.47 & $1.30 \pm 0.004$ & $0.63 \times 10^{-1}$ \\
\hline S5 & 1.47 & $1.52 \pm 0.002$ & $-\cdots$ \\
\hline S6 & 1.6 & --- & $0.584 \times 10^{-7}$ \\
\hline
\end{tabular}




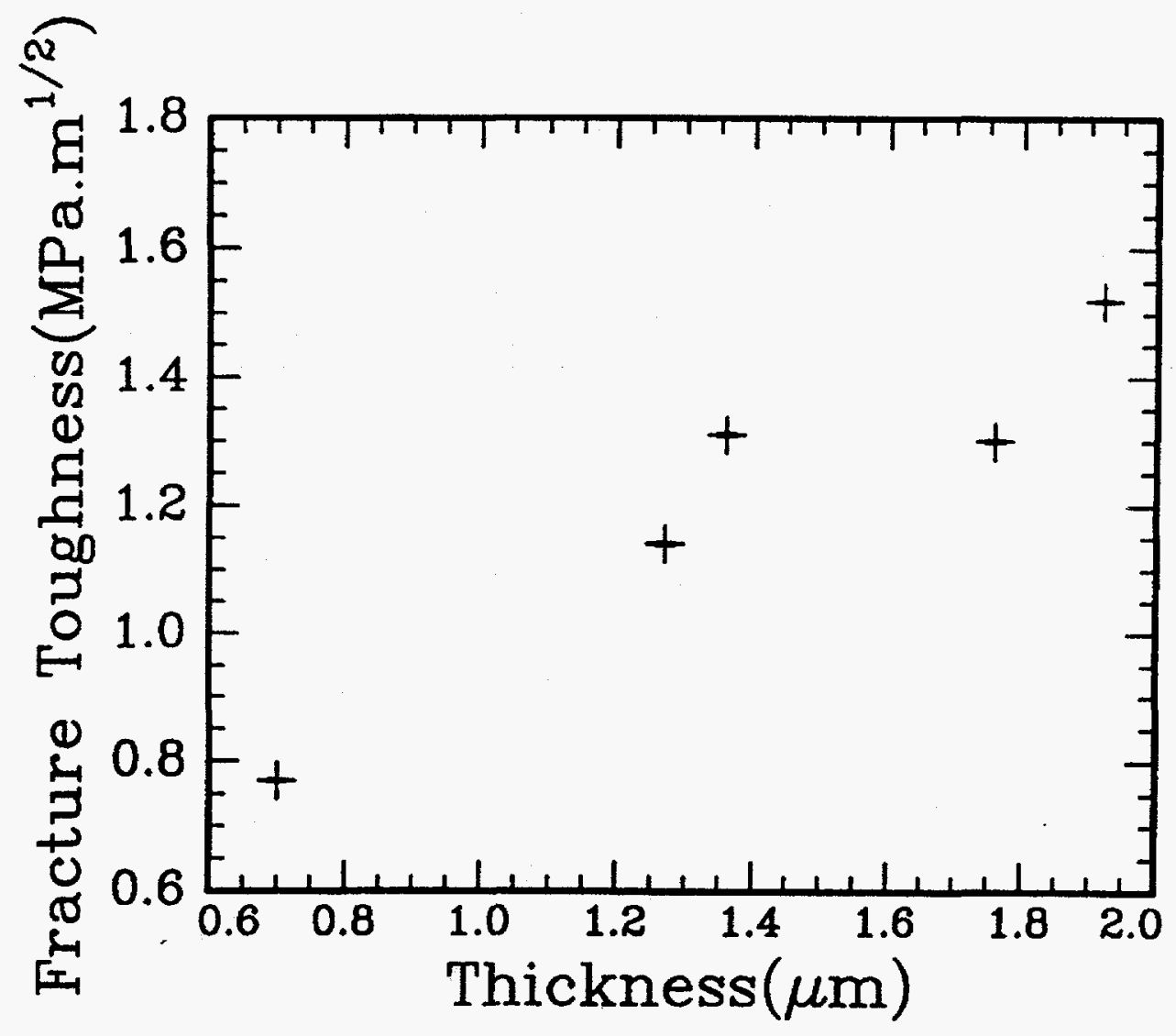

Figure 6.5 Dependence of apparent fracture toughness on coating thickness. 


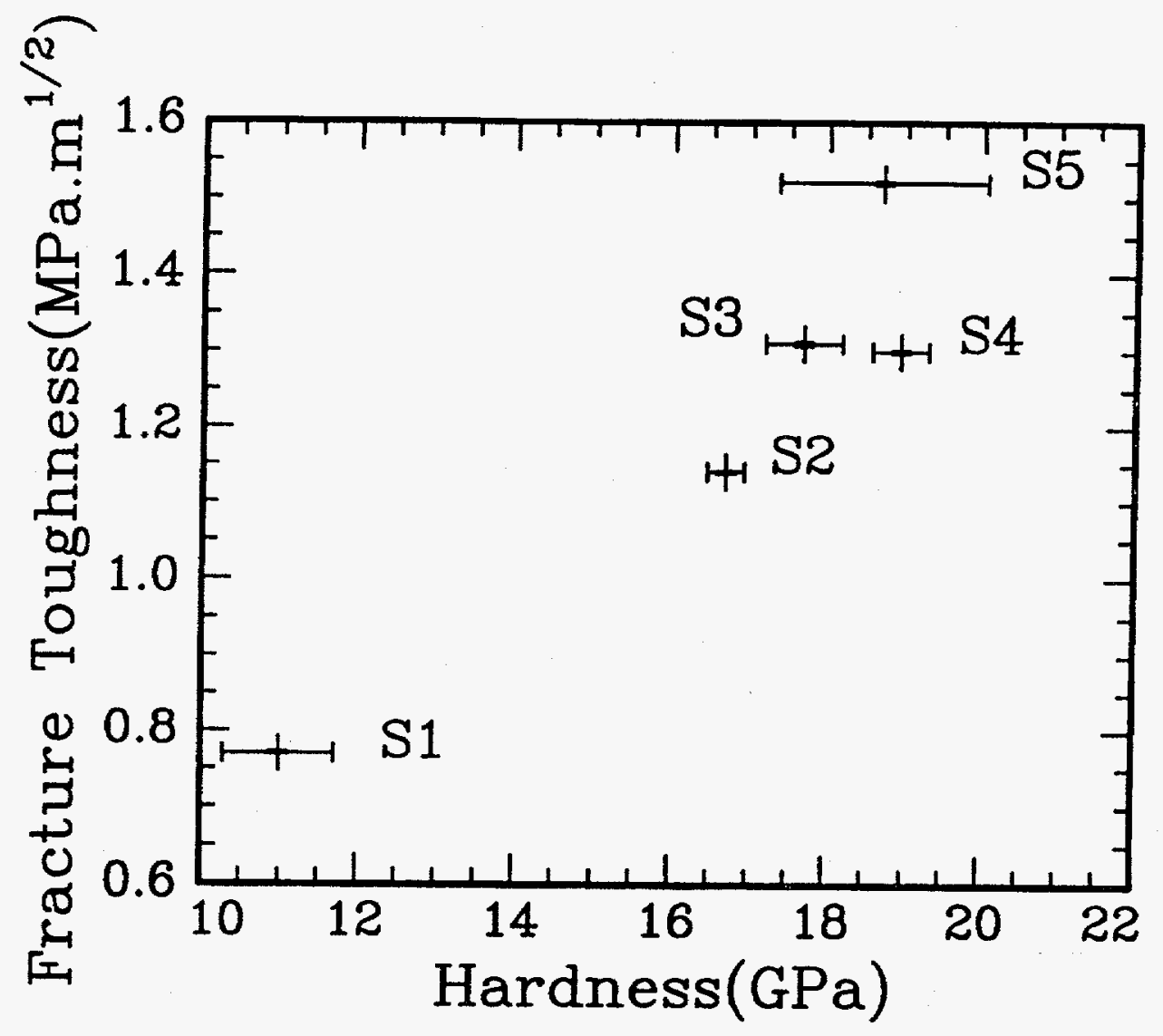

Figure 6.6 Dependence of apparent fracture toughness on hardness of the coatings. 


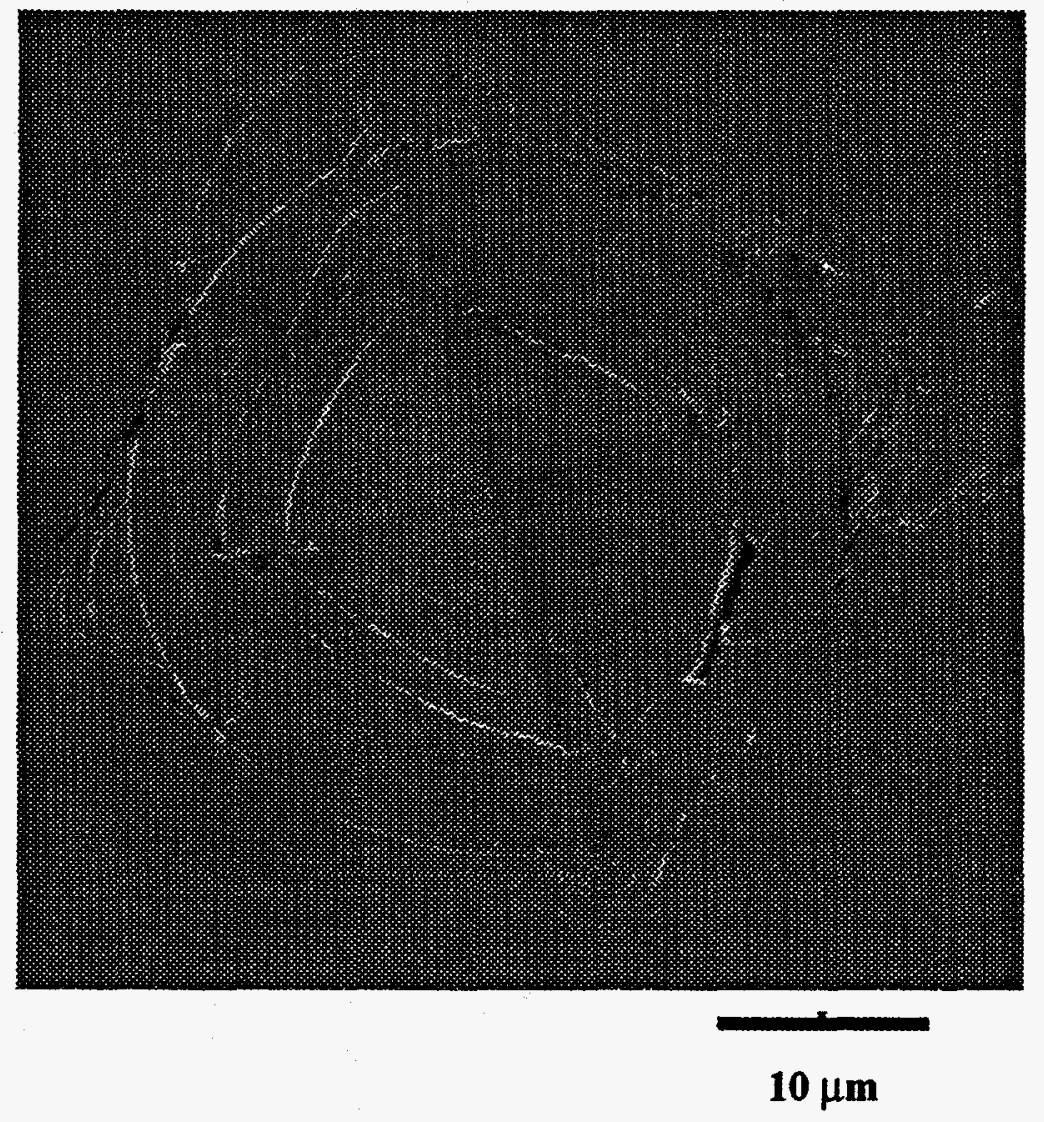

Figure 6.7 Scanning electron micrograph of the Sample S6 indented at 2000 grams. 


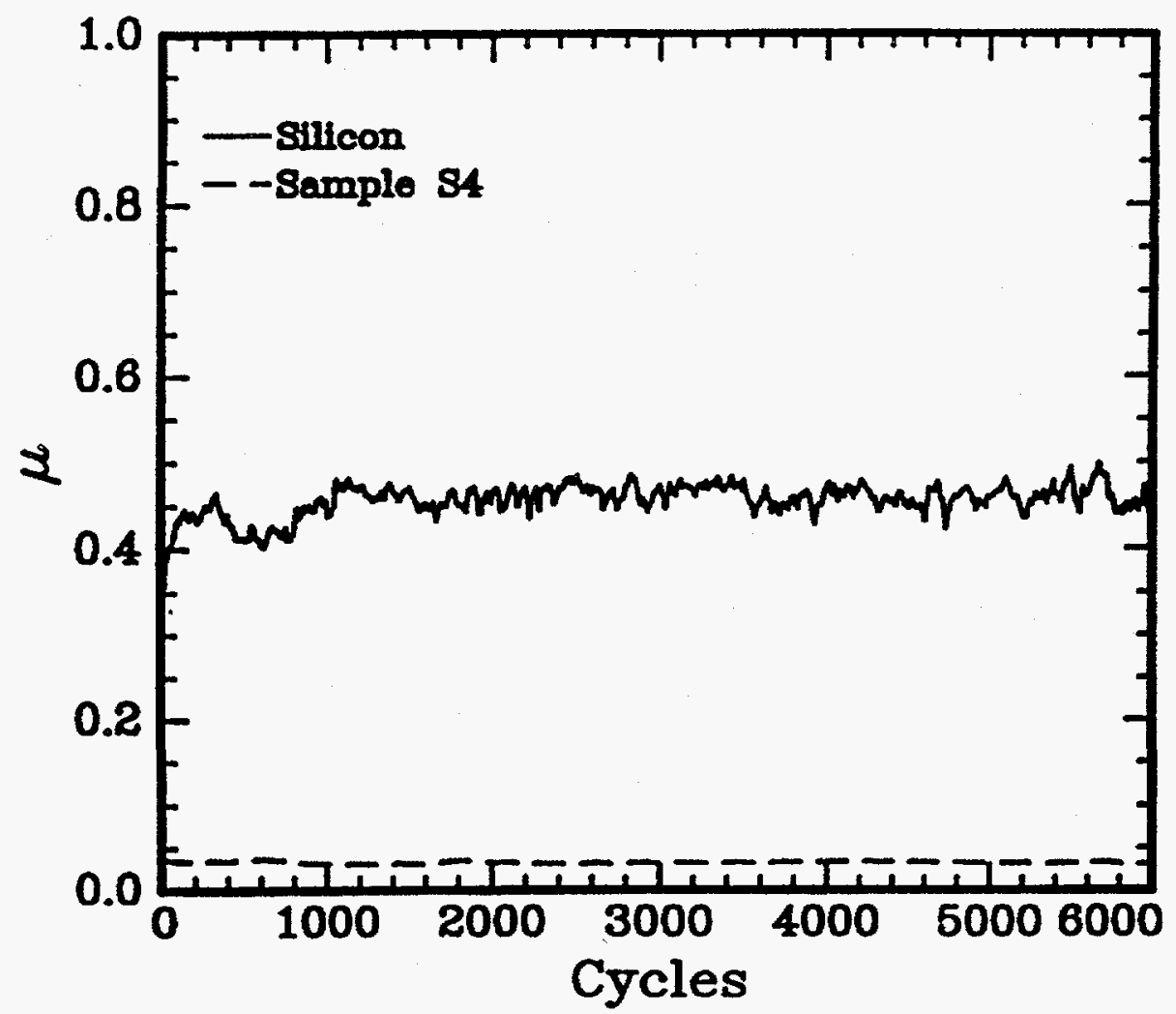

Figure 6.8 Coefficient of friction of sample 4 (S4) and uncoated silicon at $6.25 \mathrm{~N}$ with ruby pin. 
Investigations of the wear morphology for uncoated silicon and the sample S4 by scanning electron microscopy (SEM) are shown in Figures 6.9a-b. The parallel grooves running along the wear track of silicon (Figure 6.9a) indicate that abrasive wear is one of the dominant mechanism by which the silicon substrate wears. Significant wear debris and severe damage by fatigue fracture were also evident in the wear track (Figure 6.9a). Loose wear debris was also found on the pin. In contrast, for the DLC-coated samples at the same contact stress, the wear track was smooth and had no visible wear debris (Figure 6.9b). The wear morphology of the DLC-coated samples indicated that the abrasive wear mechanism found in the pure silicon has been suppressed in the coated samples. The smooth wear scar seen in the Figure $6.9 \mathrm{~b}$ suggests that wear mechansim in the coated samples may be due to plastic deformation. The ruby pins used to test DLC coatings had no visible wear. Table 6.4 shows the wear factor, Eqn. [3.14] in the units of $\left(\mathrm{mm}^{3} / \mathrm{N} . \mathrm{m}\right)$ for various samples. A comparision of the data in Table 6.3 shows that the wear factor for all the coatings scaled with apparent fracture toughness, as shown in Figure 6.10.

Figure 6.11a-c shows the wear track profiles of uncoated silicon, Samples S3 and Sample S6. Depth of the wear track of uncoated silicon was $12 \mu \mathrm{m}$ compared to the wear depth of $0.2 \mu \mathrm{m}$ for the coated samples S4 and S6. This data indicated that the coated samples wore less than $20 \%$ of the coating thickness and reduced the depth of wear track by a factor of 60 , as compared to the uncoated silicon. 

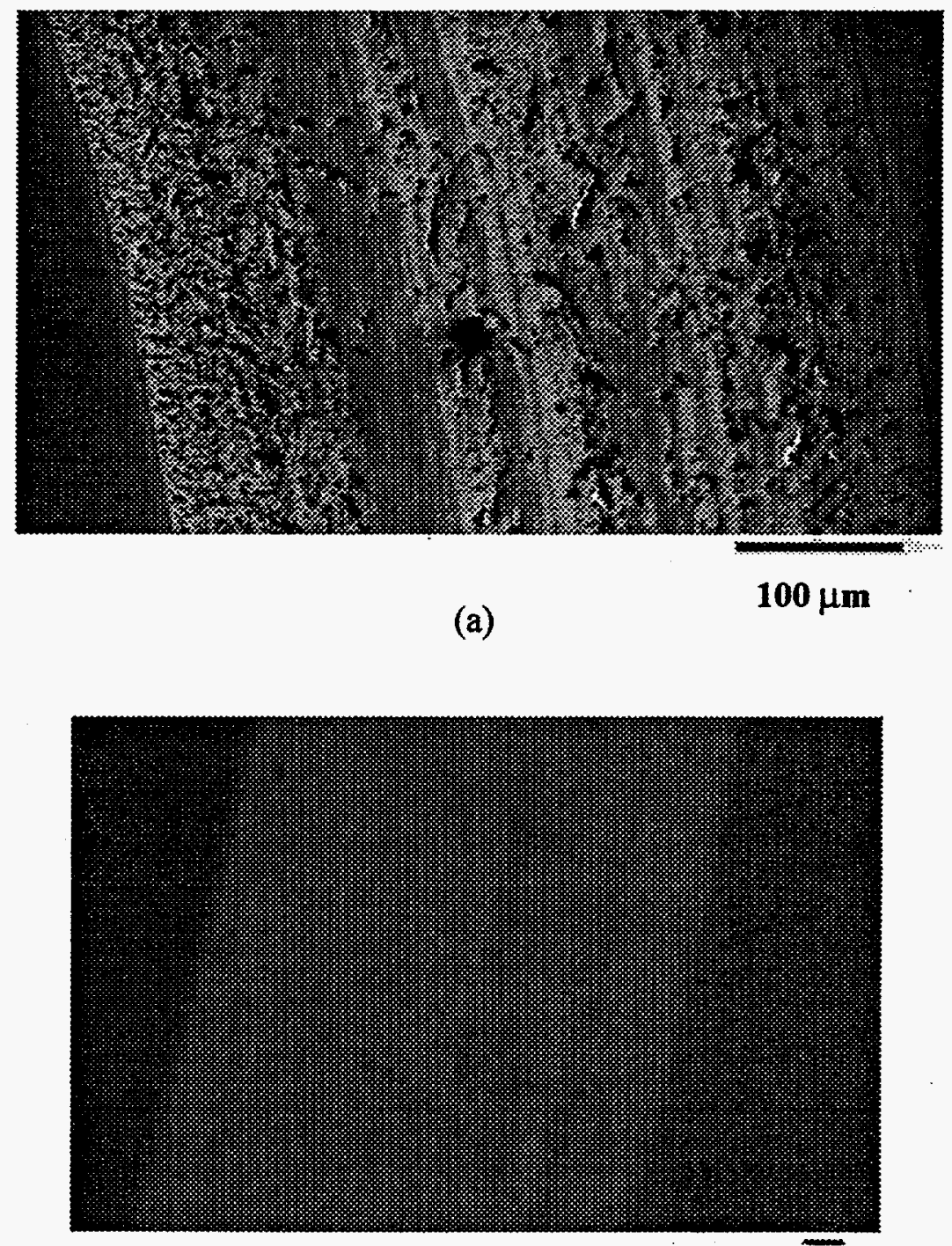

(b)

$10 \mu \mathrm{m}$

Figure 6.9 Scanning electron micrographs of the wear tracks (a) Uncoated silicon, (b) Sample S4 


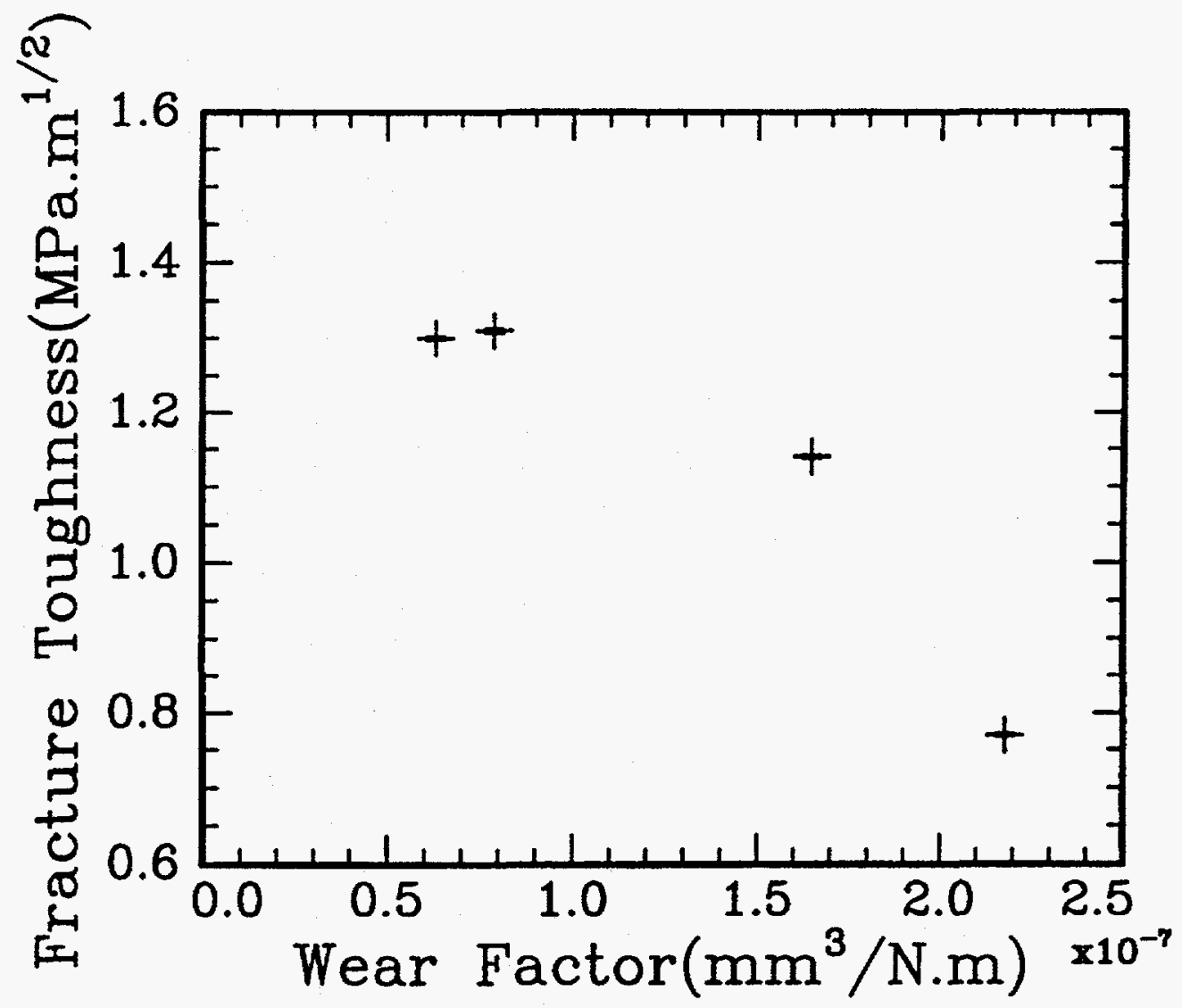

Figure 6.10 Dependence of apparent fracture toughness on wear factor. 


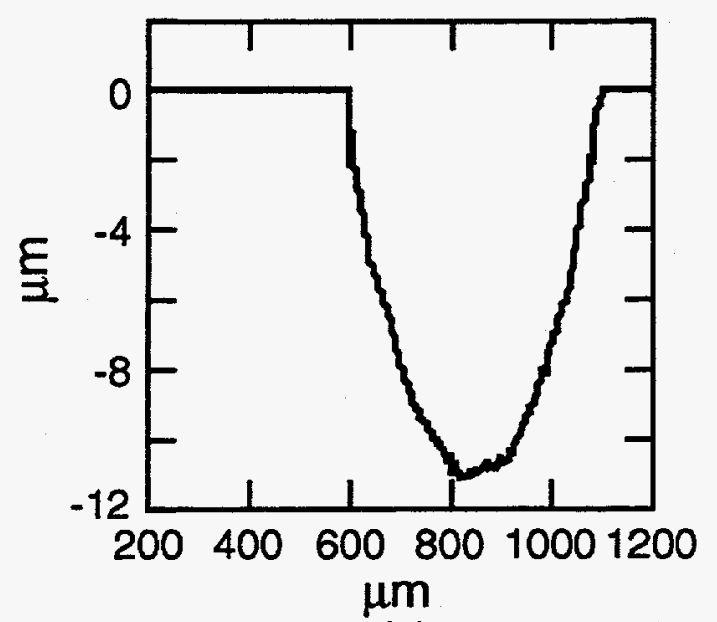

(a)

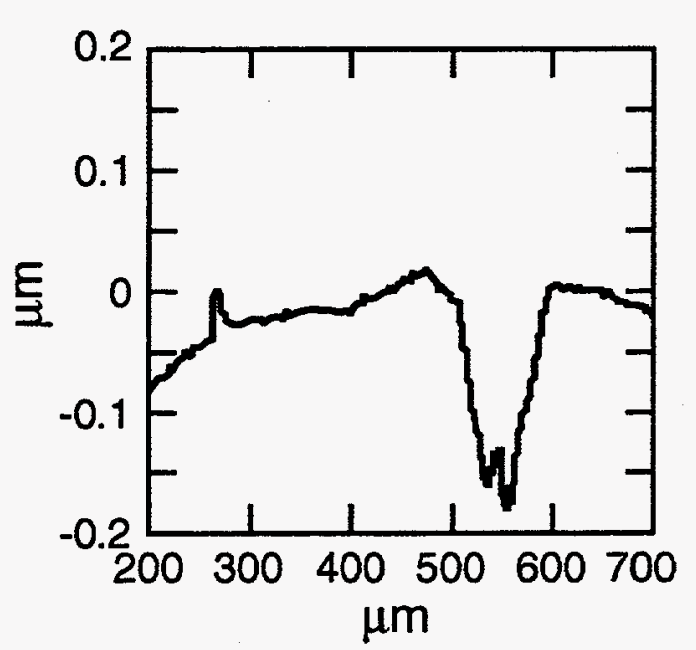

(c)

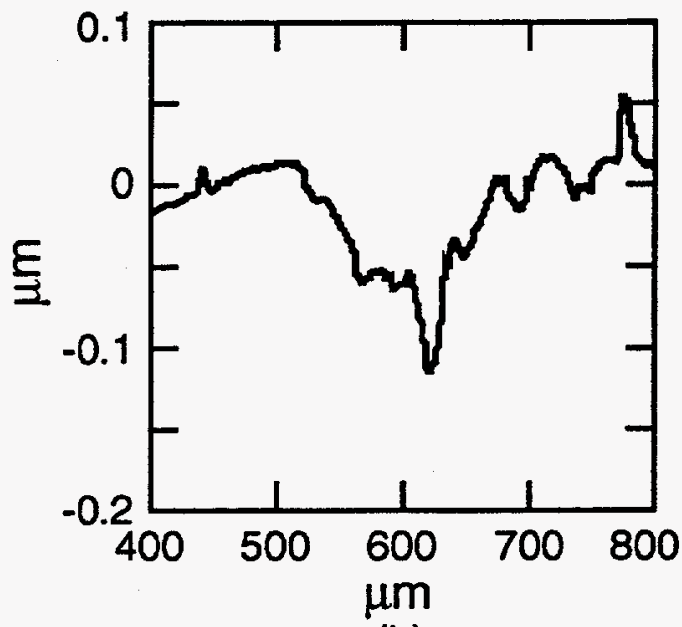

(b)

Figure 6.11. Wear track profiles of uncoated and coated samples
(a) Uncoated silicon,
(b) Sample S3,
(c) Sample S6. 


\subsection{Discussion of Results}

The discussion is divided into three sections: Surface hardness, Apparent fracture toughness, Friction and Wear behavior.

\subsubsection{Surface Hardness}

Hydrogen content has an influence on the hardness of the DLC coatings $[33,51]$. Walter et al. [53] reported hardness dependence on hydrogen content by a empirical relationship,

$$
\mathrm{H}=49.1-1.31 \mathrm{H}_{\mathrm{y}}
$$

where:

$\mathrm{H}$ is the hardness in $\mathrm{GPa}$, and

$\mathrm{H}_{\mathrm{y}}$ is the at.\% of hydrogen.

According to this relationship, hardness decreases as the hydrogen content increases. The data in Tables 6.1 and 6.2 show that the hydrogen content and hardness of the present samples follow the trend proposed by Walter et al. [33]. For example, samples (S3-S6) with a lower atomic percent of hydrogen had higher hardnesses than samples (S1-S2) with a higher atomic percent of hydrogen. A comparison of the stress data in Table 6.1 with the hardness data in Table 6.2 shows that there was no correlation between these data, in contrast to the observations of Tamor [52].

Table 6.5 summarizes the literature data for elastic moduli and hardness for carbon coatings processed by different methods. Comparing these data with the PSII processed coatings in this study (Table 6.2) indicated that the elastic moduli and hardness were comparable to the plasma deposited coatings at $100 \mathrm{~V}[151,152]$ and with the sputtered a-C coatings quoted in the literature [153].

Table 6.5 Elastic moduli and hardness values for different coatings.

\begin{tabular}{|l|c|c|c|}
\hline \multicolumn{1}{|c|}{ Material } & E, GPa & H, GPa & Ref. \\
\hline Diamond & 1050 & 103 & $147,148-150$ \\
\hline PD ac-C:H, 100 V & 145 & 16 & $151-152$ \\
\hline PD ac-C:H, 1kV & 55 & 6.3 & $151-152$ \\
\hline
\end{tabular}




\begin{tabular}{|l|c|c|c|}
\hline Sputtered a-c & 140 & 15 & 153 \\
\hline MSIB a-c & & $20-110$ & $154-155$ \\
\hline $\begin{array}{l}\text { Graphite, parallel to } \\
\text { a direction }\end{array}$ & 686 & & 149 \\
\hline Graphite, <0001> & 10 & & 149 \\
\hline Glassy C, GC10 & 29 & 3.0 & $156-157$ \\
\hline Glassy C, GC20 & 32 & 2.2 & $151-152$ \\
\hline Si & 130 & 10.4 & 149 \\
\hline a-Si:H & 100 & 10.0 & 158 \\
\hline $\begin{array}{l}\text { PD: Plasma deposited } \\
\text { MSIB: Mass selected ion beam deposition using cathodic arc. } \\
\text { a-c: amorphous carbon }\end{array}$
\end{tabular}

\subsubsection{Apparent Fracture Toughness}

Fracture toughness data showed that all five samples had a higher apparent fracture toughness than the uncoated sample. The apparent fracture toughness of these coatings decreased with hardness (Figure 6.6). Sample S6 with $7 \mu \mathrm{m}$-thickness showed no radial cracks for loads as high as $\mathbf{3 0 0 0}$ grams. Thus, sample S6 was highly fracture resistant, with a low value of compressive residual stress. The apparent fracture toughness in DLC coatings appears to be more sensitive to thickness.

From this data, it is expected that thickness, hardness, and compressive stress of the coating play different roles in the apparent fracture toughness. Thickness and hardness of the coating appear to influence the initiation of the cracks, whereas the compressive stress in the film should influence crack propagation.

It is interesting to note that the values of apparent fracture toughness observed in this study were similar to those observed by Mecholsky et al. for polycrystalline diamond coatings grown on silicon substrates [159]. Mecholsky et al. studied 6- and $12 \mu \mathrm{m}$ - thick polycrystalline diamond coatings with $55 \mathrm{MPa}$ compressive stress. In 
contrast, coating thicknesses in the present study varied between 1 and $7 \mu \mathrm{m}$, with compressive stress between 1 and $3 \mathrm{GPa}$.

As discussed earlier, residual stress, hardness, and coating thickness influence the fracture behavior of the coating-substrate system. The following discussion explains the results of apparent fracture toughness from the elastic model.

\subsubsection{Elastic Model of Fracture Toughness}

Using the energy-balance concept proposed by Griffith [160], the fracture toughness of a material can be estimated by equating the elastic energy required to fracture the surface to the surface energy required to create the two new surfaces.

$$
\mathrm{K}_{\mathrm{lC}}=\sqrt{2 \gamma \mathrm{E}}
$$

where:

$\mathrm{K}_{\mathrm{IC}}$ is the fracture toughness,

$\gamma$ is the surface energy, and

$\mathrm{E}$ is the elastic modulus.

This equation gives the minimum fracture toughness of a material; it does not include non-elastic deformation such as plastic work. Calculating the fracture toughness, $\mathrm{K}_{\mathrm{IC}}$, requires knowledge of the elastic modulus, E, and surface energy, $\gamma$. For non-

equilibrium structures, $\mathrm{K}_{\mathrm{IC}}$ can be estimated from a combination of experimental data and simple modeling. Elastic modulus values can be obtained from the nanoindentation data. Surface energy values are more difficult to obtain, especially for metastable materials.

In this discussion, surface energies are estimated using the bond energy model and the cohesive strength model. These two models are applied to single crystal diamond and silicon since experimental data for surface energies exist in the literature for these two crystals. In addition, the fracture toughness of these two crystals were also measured experimentally in the literature. Hence the estimated fracture toughness 
values from Eqn. [6.2] can be readily compared with experimental values. Use of the bond energy model and the cohesive strength model to estimate the surface energies is discussed in the following section and applied to silicon and diamond. Then, the relevant model is further applied to estimate the surface energies of amorphous DLC coatings. 


\subsubsection{Estimation of Surface Energies from Bond Energies}

Surface energy values can be estimated from bond energies and the number of surface bonds. The atoms in different planes are marked clearly by different shades of spheres in Figures 6.12 to 6.15 . Since the fracture process creates two surfaces, the surface energy is given by

$$
2 \gamma=N_{\text {sur }} \varepsilon
$$

where:

$\varepsilon$ is the bond energy between two atoms and

$\mathrm{N}_{\text {surf }}$ is the density of bonds per unit area.

$$
N_{\text {surf }}=\rho_{s} n
$$

where:

$\rho_{\mathrm{s}}$ is the surface atomic density, atoms $/ \mathrm{m}^{2}$ on the cleavage plane and

$\mathrm{n}$ is the number bonds broken per atom on one surface.

\subsection{Calculation of $\rho_{s}$, the Surface Atomic Density, on the Cleavage Plane}

Silicon and diamond have same structure. The discussion will focus on silicon and be extended to diamond. In order to calculate the surface atomic density on the cleavage plane, the number of atoms in the plane and the area enclosed by these atoms in a plane needs to be estimated. The atomic density on the cleavage plane can be calculated using the following formula,

$$
\rho_{s}=\frac{N_{p}}{A}
$$

where:

$\mathrm{N}_{\mathrm{p}}$ is the number of atoms in the plane and

$A$ is the area over which the atoms reside. 


\subsection{Calculation of the Number of Atoms in a Cleavage Plane}

The planar configuration of $\mathrm{Si}(100)$ is shown in the Figure 6.12a. The unit cell is shown by solid lines. There are 4 corner atoms shared by four unit cells, and 1 atom in the middle that is unshared. Thus, there are 2 atoms per unit cell, with area $\mathrm{a}^{2}$.

Figure 6.13a shows the planar configuration of silicon (110). The (110) plane and the unit cell in this plane are shown by the solid lines. There are 4 corner atoms shared by 4 unit cells, 2 side atoms shared by 2 unit cells, and 2 atoms unshared. Thus, there are 4 atoms in this cell, and the area in which these atoms are enclosed is $\sqrt{ } 2 \mathrm{a}^{2}$, where $\mathrm{a}$ is the cubic lattice parameter.

This analysis is repeated for the $\mathrm{Si}(111)$ plane whose planar configuration of atoms is shown in Figure 6.14a. The solid lines also represent the (111) plane and the unit cell in this plane. There are 3 corner atoms shared by 6 unit cells, and 3 side atoms shared by 2 unit cells. Thus, there are 2 atoms in this unit cell. The area of the unit in this plane that encloses these two atoms is $(\sqrt{3} / 2) \mathrm{a}^{2}$.

Table 6.6 summarizes the lattice parameters, number of atoms in the plane, area of the plane, and the atomic density for $\mathrm{Si}(100), \mathrm{Si}(110), \mathrm{Si}(111)$, and diamond (111). The data show that the atomic density of silicon in the (111) plane is less than the (110) and greater than (100) planes. In addition, the data also show that the atomic density in diamond (111) plane is greater than $\mathrm{Si}(111)$. 


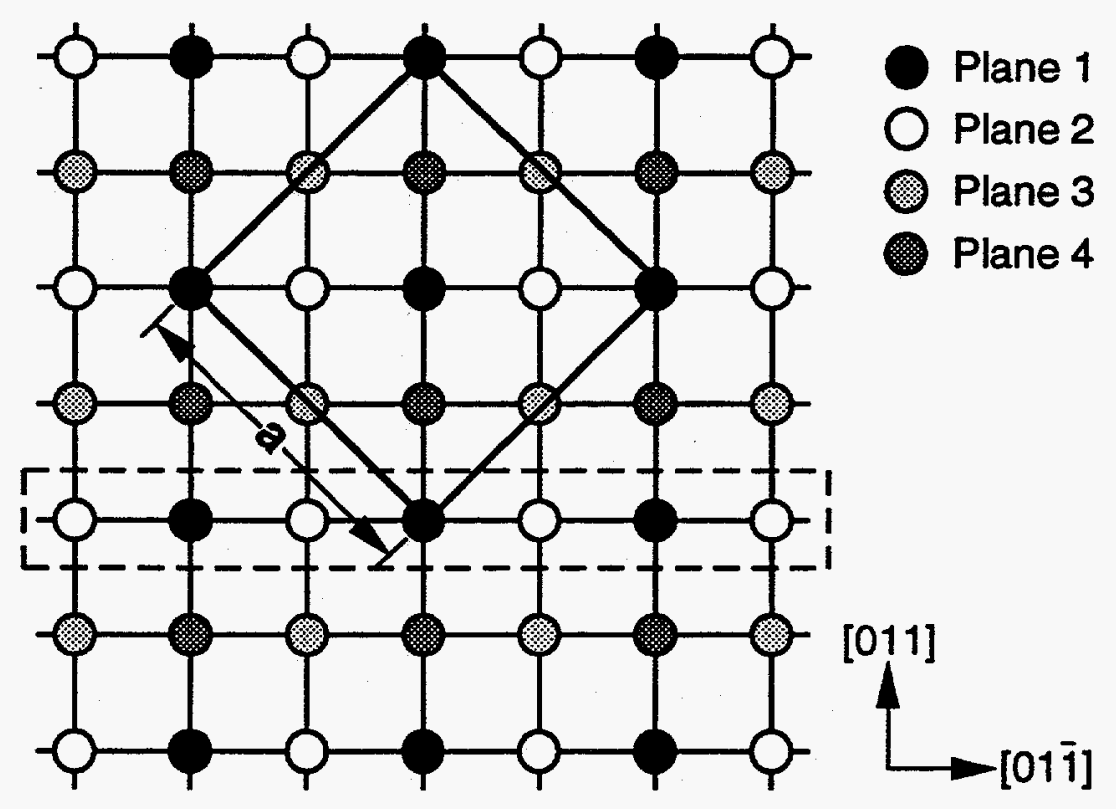

(a)

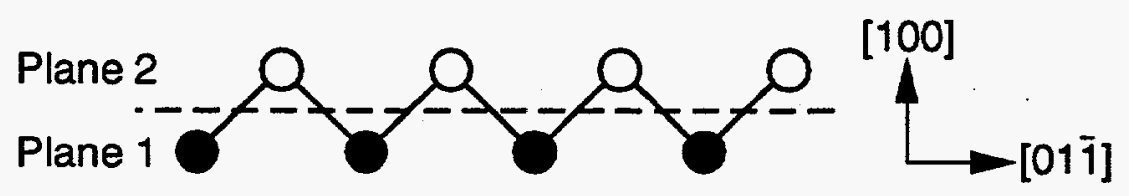

(b)

Figure 6.12a. (a) Planar atomic configuration of $\mathrm{Si}(100)$ viewed along [100].

(b) Cleavage along the face of the Si(100) between plane 1 and plane 2. 


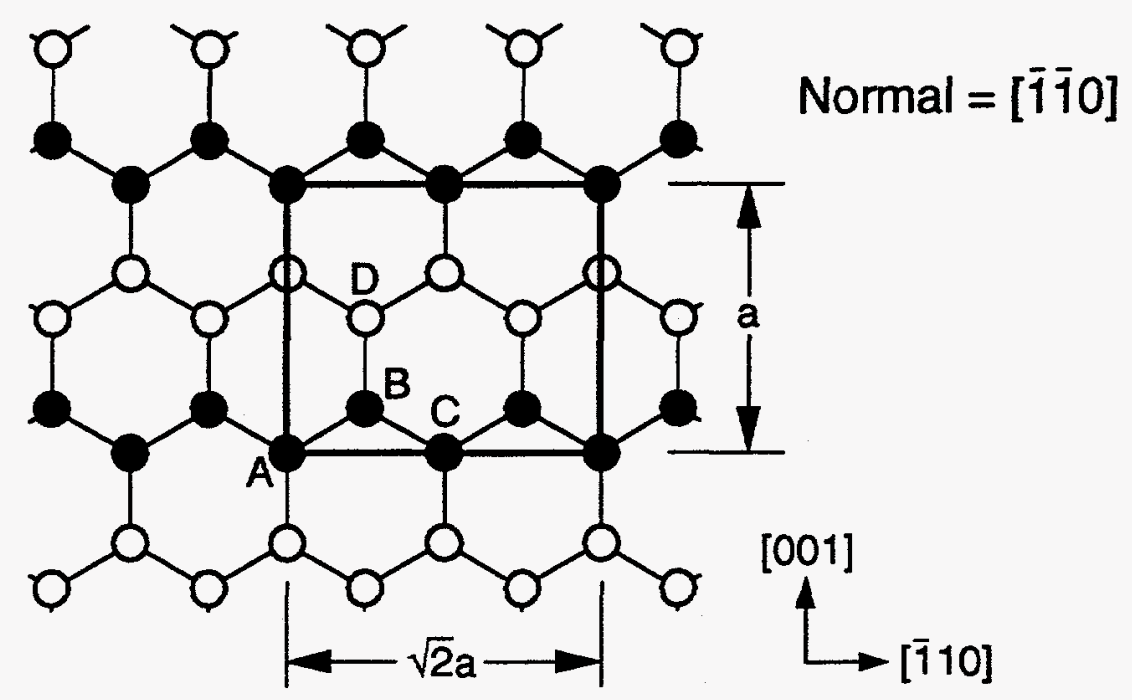

(a)

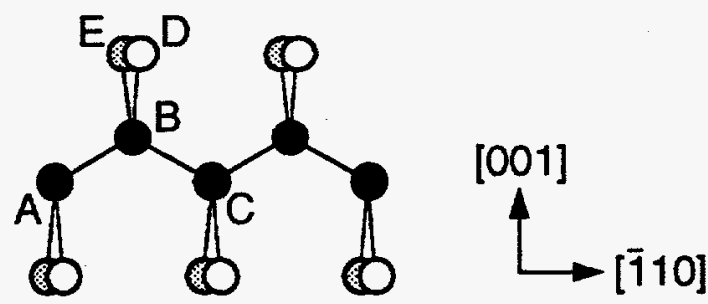

(b)

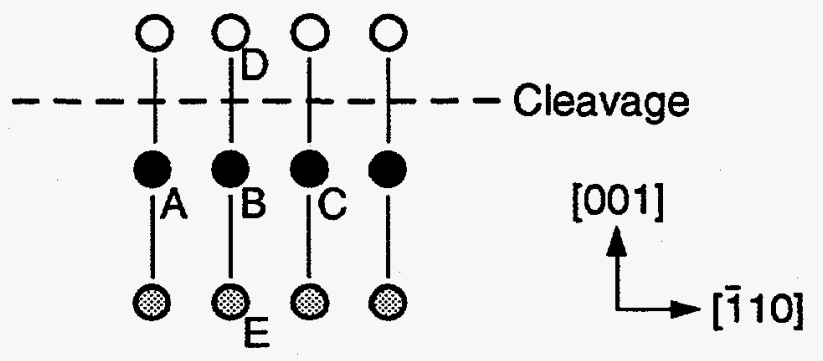

(c)

Figure 6.13. (a) Planar atomic configuration of Si(110) viewed along [110]

(b) Projected perspective view of the above configuration along $[-1,1,0]$.

(c) Projection of the figure shown in (b) along [001]. 


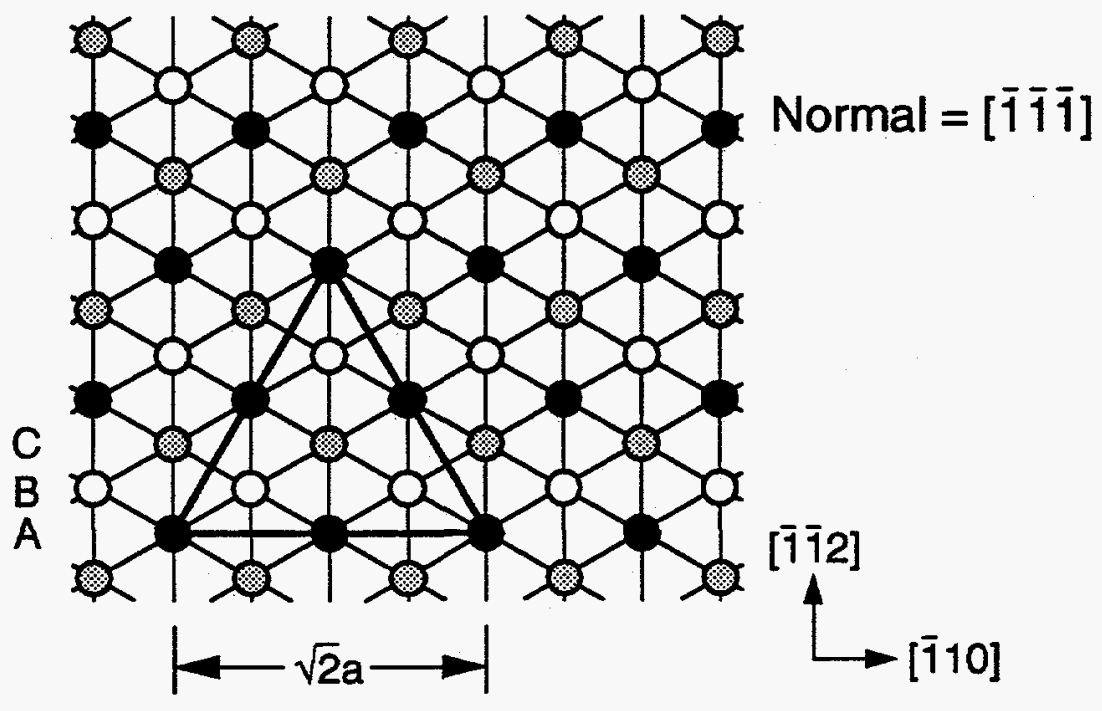

(a)

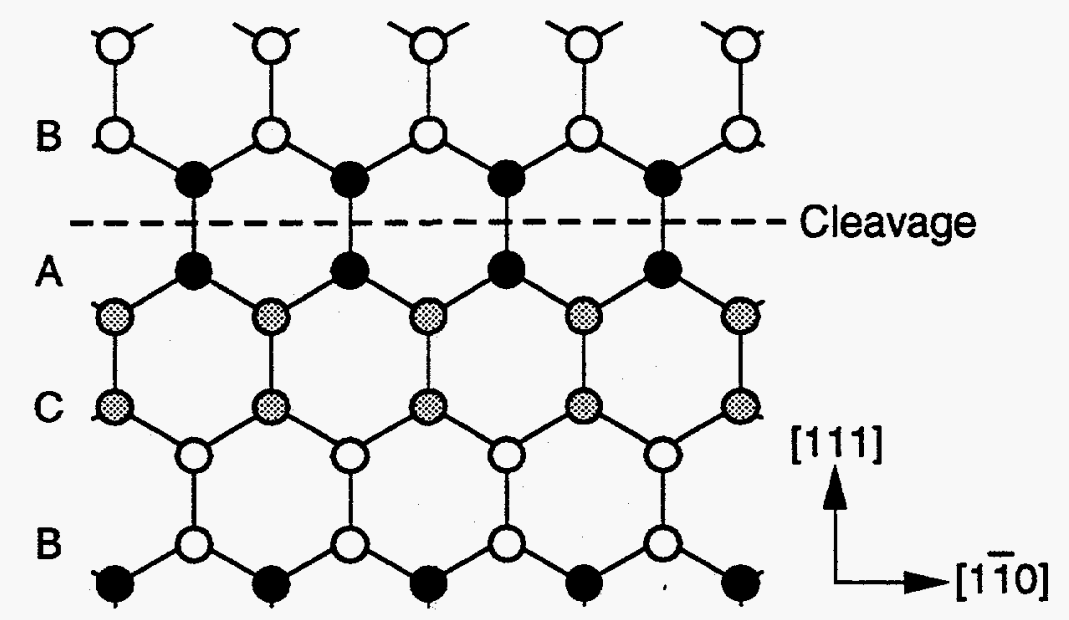

(b)

Figure 6.14a. (a) Planar atomic configuration of $\mathrm{Si}(111)$ viewed along [111].

(b) Side view of above configuration projected along $[1,1,-2]$. 


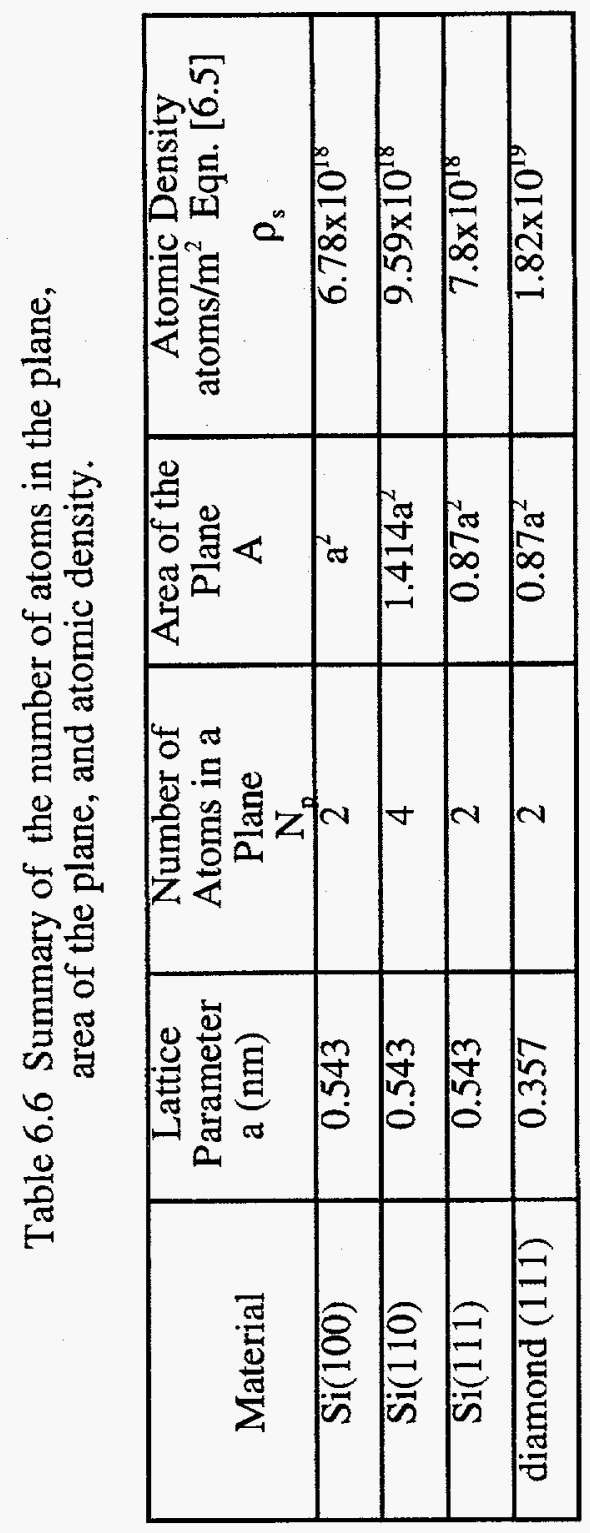




\subsection{Calculation of $n$, the Number of Bonds Broken per Atom in a Cleavage Plane}

Figure $6.12 \mathrm{~b}$ shows the cleavage along the face of the $\operatorname{Si}(100)$ between plane 1 and plane 2. From this figure, it is clear that each atom in plane 2 is connected to two atoms in plane 1 . Thus, two bonds are broken for every atom.

Figure $6.13 \mathrm{~b}$ shows the projected perspective view of the silicon $[-1,-1,0]$ direction. Black atoms (A, B, C) are coplanar. Atoms A, B, C, D, and E form a tetrahedron, with atom B at the center of the tetrahedron. Atom B is bonded to atoms $A$ and $C$ in-plane, and to atoms $D$ and $E$ in the perpendicular $(1,-1,0)$ plane. Atom $D$ is above the plane, whereas atom $\mathrm{E}$ is below the plane. Atoms $\mathrm{D}$ and $\mathrm{E}$ are shifted in this figure for atom $E$ to be visible. In order to see the stacking sequence of (110) planes, Figure $6.13 \mathrm{c}$ gives a projection along [001]. The dashed line indicates the bonds broken for cleavage. From the Figures $6.13 \mathrm{~b}-\mathrm{c}$, it is evident that one bond is broken per atom when cleaving (110).

Figure 6.14a shows the cleavage along the (111) face of the diamond structure. In the plane view of the (111) projection, the principle atoms in the cleavage plane are black and the tetrahedrally bound atoms above the principle atoms are white. In the figure, additional principle atoms reside 1 bond length below the visible black atoms. The gray atoms are tetrahedrally bonded to these invisible black atoms and reside below. Clarification of the atomic arrangement can be seen in Figure 6.14b. Rows A, $\mathrm{B}$, and $\mathrm{C}$, shown in a planar view of Figure $6.14 \mathrm{a}$, are drawn in a side view projected along $[1,1,-2]$ in Figure $6.14 \mathrm{~b}$. The easiest cleavage plane for formation of the $(111)$ surface is to break the bonds between the $\mathrm{A}$ atomic pairs. Thus, one bond is broken per every atom along (111).

After evaluating the surface atomic density, $\rho_{s}$ on the cleavage plane, and the number of bonds broken per atom on one surface, $\mathrm{n}$, the density of bonds per unit area $\mathrm{N}_{\text {surf }}$, can be calculated from Eqn. [6.4]. Table 6.7 summarizes the number of bonds 
broken per atom, atomic densities, and the bond densities for different orientations of silicon and and diamond (111) planes. The data suggests that the bond density of the Si (110) plane is greater than both (100) and (111) planes.

\subsection{Estimated Surface Energy from Bond Energies}

Once the bond densities per unit area have been estimated, surface energies can be estimated using Eqn.[6.3]. Table 6.8 summarizes the bond densities, bond energies, and estimated values of the surface energies for the $\mathrm{Si}(100), \operatorname{Si}(110), \operatorname{Si}(111)$, and diamond (111). The values of the surface energies estimated from bond energies for silicon and diamond (111) are in good agreement with experimentally measured values in the literature [161-163]. From the estimated surface energies, it is clear that the (111) plane of silicon has minimum surface energy.

There are no reports on the estimated surface energy values in the literature to compare the values estimated in this study. In cleaving a crystal along a particular plane, two surfaces are created; the surface energy of a plane is half its cleavage energy. Ramaseshan [164] estimated values of the cleavage energies of a diamond and found the cleavage energy of a (111) plane is lower than that of the (110) and (100) planes. The estimated values of surface energies in the present study follow a similar trend $\left(\gamma_{111}<\gamma_{110}<\gamma_{100}\right)$ 
Table 6.7 Summary of the number of bonds broken per atom on one surface and number of bonds per unit area.

\begin{tabular}{|l|c|c|c|}
\hline Material & $\begin{array}{c}\text { Atomic density } \\
\text { atoms/m² from } \\
\text { Table } 6.6 \\
\rho_{\mathrm{s}}\end{array}$ & $\begin{array}{c}\text { Number of } \\
\text { bonds } \\
\text { broken per } \\
\text { atom } \\
\text { on one surface } \\
\mathrm{n}\end{array}$ & $\begin{array}{c}\text { Number of bonds } \\
\text { per unit area } \\
\text { Eqn. [6.4] } \\
\mathrm{N}_{\text {surf }}\end{array}$ \\
\hline $\mathrm{Si}(100)$ & $6.78 \times 10^{18}$ & 2 & $1.36 \times 10^{19}$ \\
\hline $\mathrm{Si}(110)$ & $9.59 \times 10^{18}$ & 1 & $9.59 \times 10^{18}$ \\
\hline $\mathrm{Si}(111)$ & $7.8 \times 10^{18}$ & 1 & $7.8 \times 10^{18}$ \\
\hline diamond (111) & $1.82 \times 10^{19}$ & 1 & $1.82 \times 10^{19}$ \\
\hline
\end{tabular}




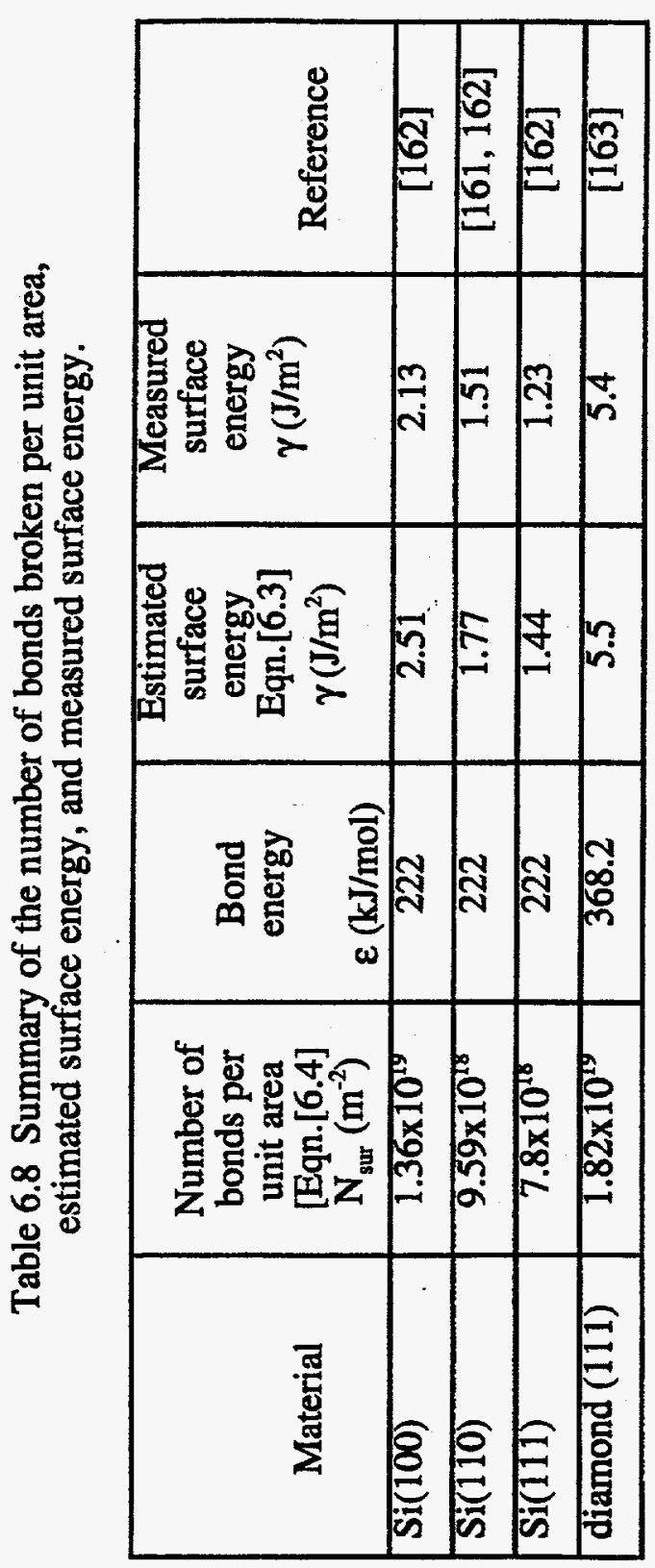




\subsubsection{Estimation of Surface Energies from the Cohesive Strength of a Material}

Surface energies have been also estimated in the literature by using a simple model by Orowan [165]. In this approach, the simple two-atom interaction potential is used to describe the fundamental interaction between atoms that are being separated to form new surfaces. The energy between atoms across the plane as a function of interatomic distance is plotted in the Figure 6.15a. At equilibrium the spacing will be $\mathrm{a}_{0}$. The force between the two atoms is simply described by $\mathrm{F}=\mathrm{dE} / \mathrm{da}$, where, $\mathrm{E}$ is the interaction energy between atom pairs and $a$ is the atomic separation. This force is plotted in Figure 6.15b as a function of interatomic distance. When atoms between two adjacent planes are separated, it is legitimate to use stress (force/area) instead of force. The restraining force per unit area (stress) that is required to separate atoms between two adjacent planes must also vary with interplanar distance as shown in Figure $6.15 \mathrm{~b}$. The work required to separate the atoms on adjacent planes from their equilibrium distance can be obtained by approximating the attractive stress, $\sigma$, between two surfaces by a sine function with period $2 \Delta \mathrm{a}$. The stress $\sigma$, is given by

$$
\sigma=\sigma_{0} \operatorname{Sin}\left[\frac{\pi}{a}\left(x-a_{0}\right)\right]
$$

where:

$\sigma_{\mathrm{o}}$ is the maximum stress,

$a_{o}$ is the equilibrium separation of the atomic planes, and

$\Delta \mathrm{a}$ is the range of interatomic force. 


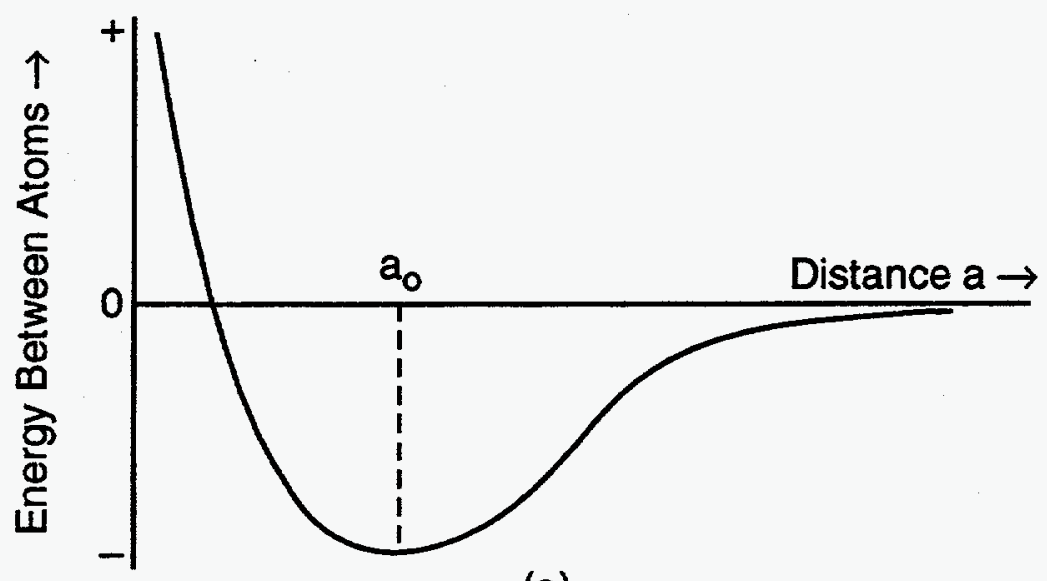

(a)

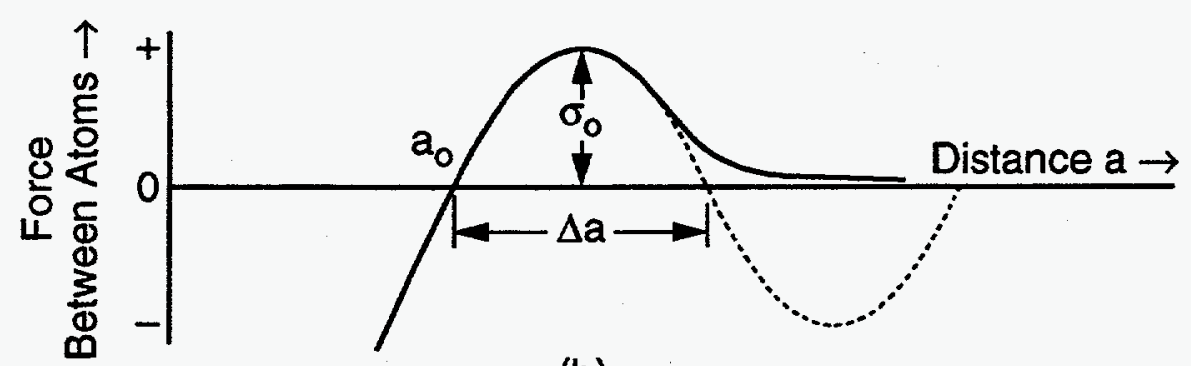

(b)

Figure 6.15 (a) Potential energy between atoms in a crystal as a function of inter-atomic distance.

(b) Force between atoms as a function of inter-atomic distance. 
At the equilibrium separation of planes, $a_{0}$, the stress is zero. With increasing separation, the stress rises to a maximum value and then drops to zero when the distance exceeds the range of attraction, a. Thus, the work done in moving the surfaces from their initial position, $a_{0}$, to a position where they are no longer attracted, can be equated to the surface energy. Orowan and Polanyi $[165,166]$ set the area under the curve equal to the surface energy of the two surfaces created.

$$
\int_{0}^{\infty+a} \sigma d x=2 \gamma
$$

where

$\gamma$ is the surface energy per unit area.

Substituting Eqn. [6.6] into Eqn. [6.7],

$$
\begin{aligned}
& \int_{a_{0}}^{a_{0}+a} \sigma d x=\int_{a_{0}}^{a_{0}+a} \sigma_{0} \sin \left[\frac{\pi}{a}\left(x-a_{0}\right)\right] d x=\frac{\sigma_{0}}{\pi} 2 a=2 \gamma \\
& \sigma_{0}=\frac{\gamma \pi}{a}
\end{aligned}
$$

It is difficult to obtain $\sigma_{o}$ directly. However, by applying Hook's law at the equilibrium distance $a_{o}$, it can be obtained:

$$
\sigma=\mathrm{E}\left(\frac{x-a_{0}}{a_{0}}\right)
$$

where $\mathrm{E}$ is the elastic modulus.

Thus, for small strains,

$$
\frac{d \sigma}{d x}=\frac{E}{\pi}\left(\frac{1}{a_{0}}\right)
$$

Differentiating Eqn. [6.6],

$$
\frac{d \sigma}{d x}=\sigma_{\circ} \frac{\pi}{a} \cos _{a} \frac{\pi}{a}\left(x-a_{0}\right)=\sigma_{\circ} \frac{\pi}{a}
$$


since $\operatorname{Cos}_{a}^{\pi}\left(x-a_{0}\right) \cong 1 . C$ when $\left(x-a_{0}\right)$ is very small.

Equating Eqn. [6.11] and [6.12],

$$
\sigma_{0}=\frac{E}{\pi}\left(\frac{a}{a_{0}}\right)
$$

Solving for the surface energy, $\gamma$, using Eqns. [6.9] and Eqn. [6.13],

$$
\gamma=\frac{E}{a_{0}}\left[\frac{a}{\pi}\right]^{2}
$$

There is large uncertainty in the application of this Eqn. [6.14] because of the difficulty in finding appropriate values for the range of attraction, a, which depends on the structure and bonding of the material. A rough estimate for surface energies can be made by assuming $\Delta \mathrm{a}$ and $\mathrm{a}_{\mathrm{o}}$ are the same order of magnitude. An alternative estimate is to equate $\Delta \mathrm{a}$, the range of interaction, with the atomic radius [161]. The equilibrium distance $a_{0}$ is taken as the bond length between atoms in the present calculations.

Surface energies estimated using this approach for diamond and silicon are summarized in Table 6.9 .

\subsubsection{Comparison of Surface Energies from Two Models}

The experimentally determined surface energy values that were collected from the literature and the estimated surface energy values from the two models discussed in the previous sections are summarized in Table 6.10. Despite the uncertainties involved in predicting surface energies, both approaches are in reasonably good agreement with the experimental values. For example, the estimated value of the surface energy for the face of a diamond (111) was in good agreement with the measured fracture energy $\gamma$, by Field and Freeman [163], which is $5.59 \pm 0.15 \mathrm{~J} / \mathrm{m}^{2}$, and theoretically predicted by Ramaseshan [164]. The largest deviation is for the estimation of the surface energy 
value for the diamond (111) plane, which is $\cong 20 \%$, from Eqn. [6.14]. This can be reduced by assuming a smaller interaction distance.

\subsubsection{Estimation of $K_{\mathrm{IC}}$ for Silicon and Diamond}

Using the estimated surface energies and the experimentally determined elastic modulus, the fracture toughness of the material can be predicted from Eqn. [6.2].

Table 6.11 compares the predicted and the experimentally determined fracture toughness values from the literature for diamond and silicon. The estimated fracture toughness values are in good agreement with the measured values of fracture toughness. For example, the fracture toughness value estimated for diamond (111) from the bond strength model and cohesive strength model are $3.7 \mathrm{MPa} \cdot \mathrm{m}^{1 / 2}$ and 4.0 MPa.m ${ }^{1 / 2}$, respectively, as compared to the experimentally measured value 3.4 MPa. $\mathrm{m}^{1 / 2}$. There are some uncertainties associated with the experimentally determined fracture toughness values. Some of them include specimen fabrication, crystal anisotropy, microstructure, and environment. 

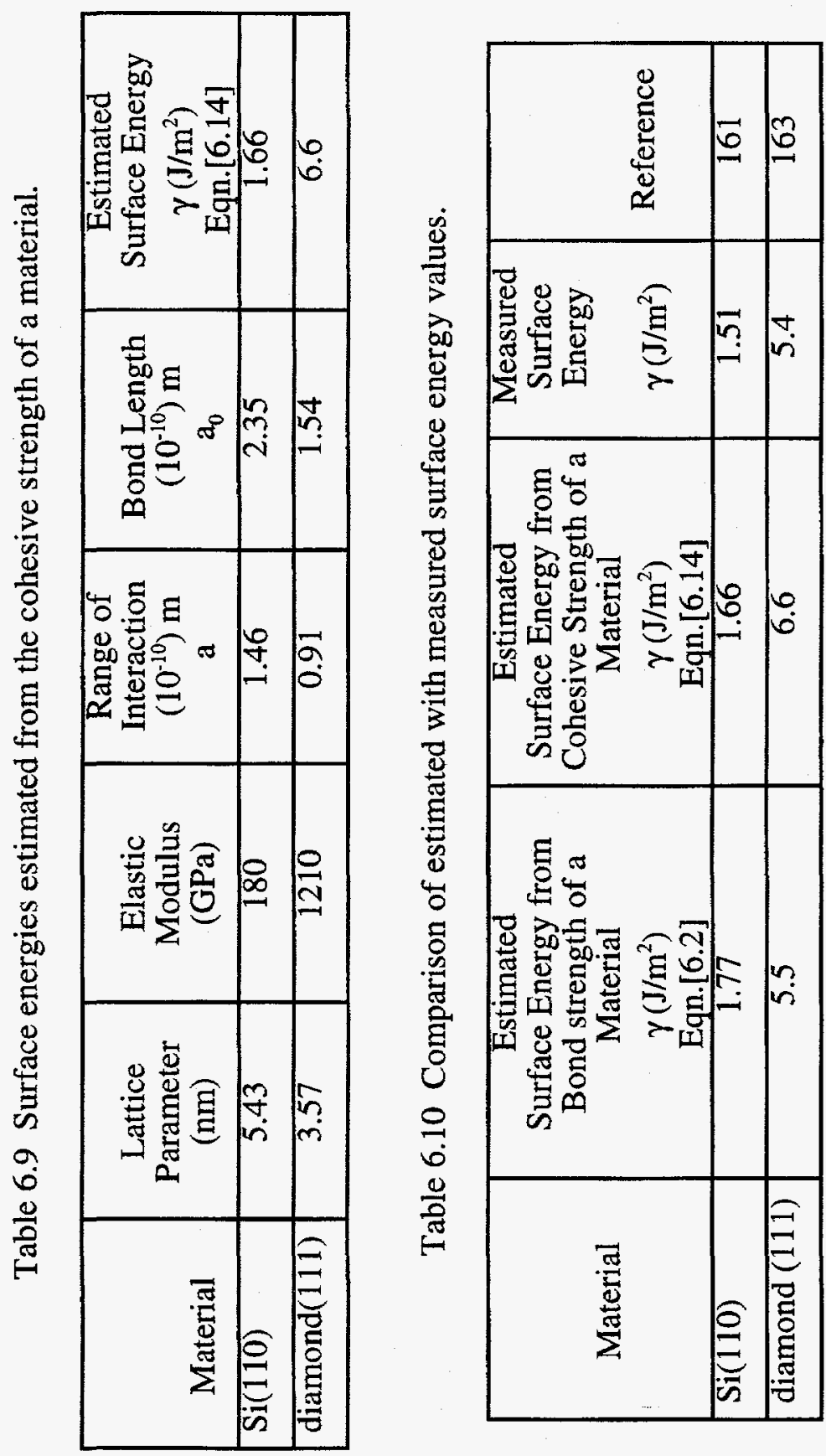
Table 6.11 Comparison of estimated fracture toughness values and measured fracture toughness.

\begin{tabular}{|l|c|c|c|c|}
\hline Material & $\begin{array}{c}\text { From Bond Strength } \\
\text { of a Material } \\
\mathrm{K}_{\mathrm{IC}} \mathrm{MPa}^{1 / 2}\end{array}$ & $\begin{array}{c}\text { From Cohesive } \\
\text { Strength of a Material } \\
\mathrm{K}_{\mathrm{IC}} \mathrm{MPa}^{1 / 2}\end{array}$ & $\begin{array}{c}\text { Experimentally } \\
\text { measured value } \\
\mathrm{K}_{\mathrm{IC}} \mathrm{MPa}^{1 / 2}\end{array}$ & Reference \\
\hline $\mathrm{Si}(100)$ & 0.8 & 0.77 & 0.7 & {$[167]$} \\
\hline diamond (111) & 3.7 & 4.0 & 3.4 & {$[149]$} \\
\hline
\end{tabular}




\subsubsection{Estimation of Minimum Value of $\mathrm{K}_{\mathrm{IC}}$ for DLC Coatings}

As mentioned earlier, to estimate the minimum fracture toughness of the amorphous DLC coatings from Eqn. [6.2], knowledge of elastic modulus and surface energy is required. Elastic modulus of these coatings can be measured by using a nanoindenter. However, estimation of surface energy for amorphous DLC coatings is not straightforward. This is due to mixed bonding $\left(\mathrm{sp}^{2}\right.$ and $\left.\mathrm{sp}^{3}\right)$ and the absence of a long-range order in the coatings. The following sections discuss the estimation of surface energy, bond energy, and density of these amorphous coatings.

\subsection{Estimation of Surface Energy of DLC Coatings}

The surface energies for DLC coatings are estimated using a similar approach to that discussed for crystalline materials in Section 6.4.2.1. The surface energy of DLC coating is given by

$$
2 \gamma_{\text {dlc }}=N_{\text {sur }} \varepsilon_{\text {DLC }}
$$

where:

$\mathrm{N}_{\text {sur }}$ is the surface atomic density and

$\varepsilon_{\mathrm{DLC}}$ is the bond energy of the DLC coating.

\subsection{Estimation of Surface Atomic Density of DLC Coatings}

The DLC coatings that were investigated in this study were amorphous, and the coatings consisted of a mixture of $\mathrm{sp}^{3}$ and $\mathrm{sp}^{2}$ bonding. Thus, in order to estimate the surface energies of the coatings, the fractions of $\mathrm{sp}^{3}$ and $\mathrm{sp}^{2}$ bonding had to be known. The fractions of $\mathrm{sp}^{3}$ and $\mathrm{sp}^{2}$ bonding in the DLC coatings that were investigated in this study by using electron energy loss spectroscopy were 0.3 and 0.7 , respectively.

It is appropriate to estimate the surface energy of the DLC coatings from bond energies and number of surface bonds. Because of the absence of a long-range periodicity in these coatings, surface bonds must be estimated without use of 
crystallography. The following assumption was used to calculate the number of bonds per unit area.

$$
N_{\text {sur }} \cong N^{\frac{2}{3}}
$$

where:

$\mathrm{N}_{\text {sur }}$ is the number atoms per unit area and

$\mathrm{N}$ is the number atoms per unit volume.

$$
N=\frac{N_{A} \rho}{A}
$$

where:

$\mathrm{N}_{\mathrm{A}}$ is Avagadro's number,

$A$ is the atomic mass number, and

$\rho$ is the density.

\subsection{Determination of $\rho$, the Density of DLC Coating}

Assuming that the DLC coating was uniform and had only carbon and hydrogen, the areal density (atoms $/ \mathrm{cm}^{2}$ ) was determined using $3.55 \mathrm{MeV} \mathrm{He}$ ion beam RBS data. This areal density can be converted to volume density (atoms $/ \mathrm{cm}^{3}$ ) by using Eqn. [6.18]. The thickness of the film was measured by profilometry. The simulated spectra using RUMP [124] gives the atomic fraction of carbon and hydrogen and the coating density in atoms $/ \mathrm{cm}^{2}$. The coating density in $\left(\mathrm{gm} / \mathrm{cm}^{3}\right)$ was calculated using the following expression.

$$
\rho\left(\frac{\mathrm{gm}}{\mathrm{cm}^{3}}\right)=\frac{\text { atoms }}{\mathrm{cm}^{2}} \frac{\left[\mathrm{f}_{\mathrm{c}} \mathrm{A}_{\mathrm{c}}+\hbar_{h} A_{h}\right]}{N_{\mathrm{A}}}
$$

where:

$\rho$ is the mass density of the coatings $\left(\mathrm{gm} / \mathrm{cm}^{3}\right)$,

$\mathrm{t}$ is the thickness of the coatings $(\mathrm{cm})$, measured using profilometry,

$f_{c}$ is the atomic fraction of carbon,

$f_{h}$ is the atomic fraction of hydrogen, 
$A_{c}$ is the atomic weight of carbon,

$A_{b}$ is the atomic weight of hydrogen, and

$\mathrm{N}_{\mathrm{A}}$ is the Avagadro's number.

The average density of these coatings was determined to be $2.67 \mathrm{~g} / \mathrm{cm}^{3}$. This is in agreement with the values estimated by Ojha et al. for $\mathrm{H} / \mathrm{C}$ ratio of 0.42 [168].

ERD measurements indicated $30 \%$ hydrogen and $70 \%$ carbon. Assuming that all the hydrogen atoms present in the coatings were attached to carbon atoms in $\mathrm{sp}^{3}$ bonding, $43 \%$ would be $\mathrm{C}-\mathrm{H}$ molecules. The remaining $57 \%$ of carbon would be in $\mathrm{sp}^{2}$ bonding. This result was in agreement with EELS measurements (which were not discussed here) that were performed on some of the coatings.

Using the density $\rho$ in Eqn. [6.18], the number of atoms per unit volume (carbon and hydrogen) are $1.23 \times 10^{29}$ atoms $/ \mathrm{m}^{3}$. The estimated number of surface atoms is $1.6 \times 10^{19}$ atoms $/ \mathrm{m}^{2}$. Thus, the number of surface atoms calculated in DLC coatings is same order of magnitude as in a closed packed plane of a diamond (Table 6.6).

In Section 6.4.2.2.3, it was shown that cleavage along the $\mathrm{Si}(100)$ plane results in breaking two bonds per atom. Since silicon and diamond have the same structure, two bonds are also broken for the diamond (100) plane. ). Assuming two bonds are broken in cleaving a DLC coating, the number of bonds per unit area in a coating are much higher than those in the closed packed plane of diamond (111). On other hand, assuming the one bond is broken per one atom in DLC coating, as in (110) and (111) planes, the number of surface bonds in DLC coatings is same as the number of surface atoms. The absolute number of surface bonds in the DLC coating, $1.6 \times 10^{19}$ atoms $/ \mathrm{m}^{2}$, is less than the surface bonds for the closed packed plane of diamond, $1.82 \times 10^{19}$ atoms $/ \mathrm{m}^{2}$. This appears to be reasonable. 


\subsection{Estimation of Bond Energy of DLC Coatings}

The DLC coatings that were investigated in this study were amorphous, and the coatings consisted of a mixture of $\mathrm{sp}^{3}$ and $\mathrm{sp}^{2}$ bonding. Thus, the average bonding energy of DLC coatings investigated can be estimated using the following equation.

$$
\varepsilon_{\mathrm{DLC}}=\mathrm{F}_{\mathrm{sp}} \varepsilon_{\mathrm{sp}}+\mathrm{F}_{\mathrm{sp^{2 }}} \varepsilon_{\mathrm{sp}}
$$

where :

$\mathrm{F}_{\mathrm{sp}^{3}}$ is the fraction of $\mathrm{sp}^{3}$ material $=0.3$,

$\mathrm{F}_{\mathrm{sp}}$ is the fraction of $\mathrm{sp}^{2}$ material $=0.7$,

$\varepsilon_{\mathrm{sp}^{3}}$ is the bond energy of $\mathrm{sp}^{3}=368 \mathrm{~kJ} / \mathrm{mol}$, and

$\varepsilon_{\mathrm{sp}^{2}}$ is the b energy of $\mathrm{sp}^{2}=638 \mathrm{~kJ} / \mathrm{mol}$.

Thus, the bond energy of DLC coatings, $\varepsilon_{\mathrm{DLC}}$, is $557 \mathrm{~kJ} / \mathrm{mol}$.

\subsubsection{Estimation of Surface Energy of DLC Coatings}

Using the bond energy values estimated in the previous section, and the number of surface bonds in Section 6.4.2.6.3, the predicted surface energy of DLC coatings is found to be $7.4 \mathrm{~J} / \mathrm{m}^{2}$. This value of surface energy was approximately $30 \%$ greater than the estimated or experimentally measured value for diamond (111) plane. As mentioned in the beginning of this discussion, the surface energies for metastable phases are not available in the literature. Thus, comparison can only be made with respect to diamond. There are experimental errors in measuring the percent of $\mathrm{sp}^{2}$ and $\mathrm{sp}^{3}$, which could decrease or increase the estimated surface energy value of the coating.

\subsection{Estimation of the Fracture Toughness of DLC Coatings}

Using the elastic modulus values obtained from nanoindentation measurements, and the surface energy estimated in the previous section, the fracture toughness of the coating can be estimated using Eqn. [6.2]. These values were 
summarized in Table 6.12. The estimated values are higher than the silicon substrate. The estimated values cannot be compared directly with the experimentally measured apparent fracture toughness values using indentation technique that are also summarized in Table 6.12. The experimentally measured values of the fracture toughness includes substrate effects, whereas the estimated value is for the coating alone. However, estimation of the surface energy of the coating-substrate system is done using the simple approximations in the following section. 


\subsection{Estimation of the Apparent Fracture Toughness of DLC-Si System}

Estimation of the fraction of the substrate and coating contribution to the surface energy can be done, since the crack lengths of the coating-substrate system at a particular load are known. The highest load used was 1000 gms, and the lowest load at which the well-defined cracks were formed was 300 gms.

The length of the cracks in the coating-substrate system at $1000 \mathrm{gms}$ and 300 gms were $52.2 \mu \mathrm{m}$ and $17 \mu \mathrm{m}$, respectively. Figure 6.16 shows the half-penny crack of a coating-substrate system. The area of the crack is $\pi \mathrm{c}^{2} / 2$, where $\mathrm{c}$ is the crack length. The area of the coating, $A_{D L C}$, can be approximated to $2 \mathrm{~cd}$, where $d$ is the coating thickness. The substrate contribution to the area of the crack, $A_{\mathrm{s}}$, is given by

$$
\begin{aligned}
& A_{S i}=\frac{\pi r^{2}}{2}-2 r d \\
& A_{t o t}=A_{S i}+A_{D L C}
\end{aligned}
$$

The surface energy of a coating-substrate system is approximated by

$$
\gamma_{\text {tot }}=\frac{A_{D L C}}{A_{\text {tot }}} \gamma_{D L C}+\frac{A_{S i}}{A_{\text {tot }}} \gamma_{S i}
$$

Using, Eqn. [6.22], the surface energy of the coating-substrate system is estimated to be $1.85 \mathrm{~J} / \mathrm{m}^{2}$ and $2.2 \mathrm{~J} / \mathrm{m}^{2}$, at $1000 \mathrm{gms}$ and $300 \mathrm{gms}$, respectively. The fracture toughness of the coating-substrate system was estimated, using Eqn. [6.2] and by approximating coating modulus to be equal to the substrate modulus. The apparent fracture toughness of the coating-substrate system was found to be $0.82 \mathrm{MPa} \cdot \mathrm{m}^{1 / 2}$ and $0.9 \mathrm{MPa} \cdot \mathrm{m}^{1 / 2}$, at $1000 \mathrm{gms}$ and $300 \mathrm{gms}$, respectively. 


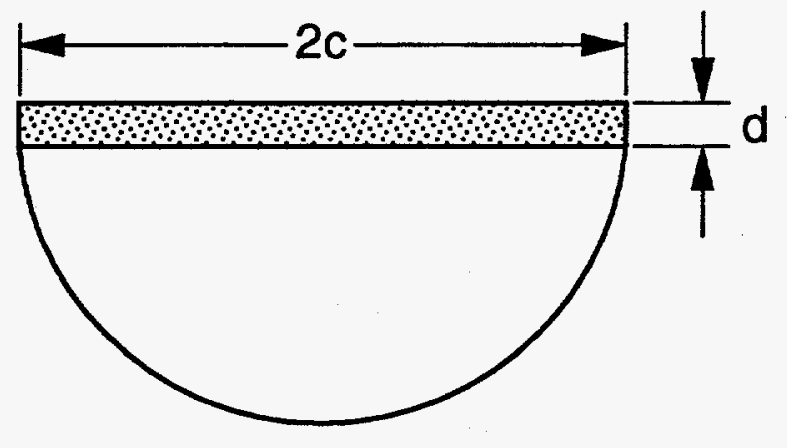

Figure 6.16. Half-penny crack of a coating-substrate system viewed in cross-section. 
The estimated value of the apparent fracture toughness is less than the experimentally measured values. This discrepancy could be due to intrinsic residual stress as result of processing and/or due to plastic deformation of the coating-substrate system.

Table 6.12 Predicted and experimentally determined fracture toughness.

\begin{tabular}{|l|c|c|c|}
\hline Sample & $\begin{array}{c}\text { Elastic Modulus } \\
(\mathrm{GPa})\end{array}$ & $\begin{array}{c}\text { Predicted } \mathrm{K}_{\mathrm{IC}} \text { of } \\
\text { the coating }_{\left(\mathrm{MPa} \mathrm{m} \mathrm{m}^{1 / 2}\right)} \\
\text { Eqn. [6.2] }\end{array}$ & $\begin{array}{c}\text { Experimentally } \\
\text { Determined } \\
\text { values of } \mathrm{K}_{\mathrm{IC}} \\
\left(\mathrm{MPa} \mathrm{m}^{1 / 2}\right) \\
\text { Eqn. [3.15] }\end{array}$ \\
\hline $\mathrm{Si}(100)$ & 180.0 & 0.67 & $0.67 \pm 0.001$ \\
\hline $\mathrm{S} 1$ & $113.6 \pm 4.73$ & 1.12 & $0.77 \pm 0.002$ \\
\hline $\mathrm{S} 2$ & $140.7 \pm 1.43$ & 1.25 & $1.14 \pm 0.002$ \\
\hline $\mathrm{S} 3$ & $145.8 \pm 2.55$ & 1.27 & $1.31 \pm 0.003$ \\
\hline $\mathrm{S} 4$ & $158.9 \pm 2.07$ & 1.33 & $1.30 \pm 0.004$ \\
\hline S5 & $159.9 \pm 11.5$ & 1.33 & $1.52 \pm 0.002$ \\
\hline S6 & $188.9 \pm 3.48$ & 1.45 & --- \\
\hline
\end{tabular}

\subsubsection{Friction and Wear Behavior}

Low friction of the DLC coatings observed in this investigation was consistent with reported values in the literature [32-36, 169-172]. Abrasive wear is the dominant wear mechanism in silicon. Under the same contact stress, DLC-coated samples wear by plastic deformation. Wei et al. [170, 171] and Liu et al. [172] reported formation of graphite on DLC-coated disc after a 100 million cycle rolling contact fatigue. Though there is no direct evidence of formation of a thin graphitic layer in the current tests, it is speculated that graphitization is a possible mechanism for a low frictional behavior of the coated samples.

The wear factor of the silicon substrate is several orders of magnitude greater than the wear factors of any of the DLC coatings investigated in this study. The wear 
factor for all the coatings, scaled with apparent fracture toughness, was calculated using an indentation technique. As mentioned in Chapter 2, several theoretical models relating hardness, fracture toughness, and wear exist in the literature [79-83]. These models are applied to specific types of wear, such as severe abrasive wear, which includes microcracking, microploughing, and microcutting or erosive wear. As discussed earlier, no visible debris in the wear track in Figure $6.9 \mathrm{~b}$ suggested that the wear behavior in the coated samples was not abrasive. The wear behavior of the coatings relative to the uncoated silicon was improved because of their low coefficient of friction and high fracture toughness.

The static contact stress between silicon and the ruby slider, calculated using Eqn. [2.6], was about 1.0 GPa in this investigation, whereas the yield strength of silicon is about $5 \mathrm{GPa}$ [173]. Tables 6.2 and Table 6.4 show that the contact stress on DLC was much less than the hardness. This suggested that, microscopically, the wear should not be dominated by plastic deformation or fracture.

The detailed contact mechanics of a sphere loaded elastically against a plane were discussed in Chapter 2. The tangential traction caused by friction between the pin and the disc also modifies the contact stress distribution over the static case [90, 91]. Once sliding begins and frictional forces operate, the region in front of the sliding contact is in compression, whereas the region behind the contact is in tension. As shown in the Eqn. [2.16], deviation in the stress from the static contact condition scales with the coefficient of friction. The cyclic loading that results from the change in the stress as the pin slides across the surface can lead to a fatigue fracture condition on the disc surface. Points subjected to tensile stresses in the system are generally more susceptible to this type of failure.

Arnell [174] has discussed that deformation caused by repeated loading can form wear debris below the fatigue limit. The wear morphology of the uncoated silicon (Figure 6.9a) indicated wear particles with sharp edges and cracks. No transfer 
layer was observed in the wear track, suggesting the wear indeed was due to fracture. Fatigue fracture is directly related to the magnitude of the tensile stress in the system. This cyclic failure can be suppressed if the coefficient of friction and the resulting tensile stress of the system under study is low. The coatings deposited on the silicon substrates had low coefficients of friction. As a result, the compressive and tensile surface stresses that resulted (Figure 2.7) because of the sliding contact were minimized and thus reduced the probability of fracture by fatigue.

This research revealed a possible linear relation between the two mechanical properties (hardness and fracture toughness) and the wear factor. The sample S5 and uncoated silicon had the same hardness; however, their wear rates differed by several orders of magnitude. This observation illustrated that high apparent fracture toughness and a low coefficient of friction were responsible for the wear-resistant nature of the DLC coatings.

\subsection{Conclusions}

Hardness of DLC coatings with less than 30 atomic percent hydrogen was found to be in the range of $11 \mathrm{GPa}$ and $19 \mathrm{GPa}$. No correlation was observed between hardness and residual stress in the coatings. The coating hardness increased with coating thickness.

The apparent fracture toughness measured using the indentation technique was found to be higher than the estimated value using the Griffith model for elastic fracture. Three main factors can explain this discrepancy: (i) Residual stresses due to processing (ii) plastic deformation during indentation (iii) both (i) and (ii). The exact contribution of each of these factors couldn't be estimated. This study suggested that coating-substrate fracture toughness using indentation technique can be correlated with the other mechanical properties of the coating. The apparent fracture toughness of these coatings was found to be similar to the values reported for polycrystalline diamond films [147]. 
DLC coatings, tested at a contact stress of about $1 \mathrm{GPa}$, were found to have low friction (0.1) and low wear factors $\left(10^{-7} \mathrm{~mm}^{3} / \mathrm{N} . \mathrm{m}\right)$. The data suggested that the loss of material in DLC coatings is due to plastic deformation. Though there is no direct evidence of formation of a thin graphitic layer in these tests, it is speculated that graphitization is a possible mechanism for a low frictional behavior of the coated samples. The wear-resistant nature of the DLC coatings was attributed to high apparent fracture toughness and a low coefficient of friction.

In summary, strong adherent amorphous diamond-like carbon coatings with good tribological and mechanical properties were produced using PSII. The apparent fracture toughness of amorphous DLC coatings were evaluated for the first time in the literature. 


\section{Chapter 7 Investigations of BCN Coatings}

Hard, wear-resistant coatings often fail by brittle fracture because of their low fracture toughness. One method to improve the performance of these coatings is to add a softer phase to a hard coating, which can improve fracture toughness. The high hardness and wear resistance of boron carbide and the lubricating nature of crystalline hexagonal boron nitride, also known as white graphite, are known in the literature [54]. The hardnesses of polycrystalline boron carbide and hexagonal boron nitride are $40 \mathrm{GPa}$ and $9 \mathrm{GPa}$, respectively [54]. The structure and the sensitivity of a hexagonal boron nitride to the presence of condensable vapors are similar to graphite.

The idea to combine the properties of boron carbide and hexagonal boron nitride for a potential wear-resistant coating was explored by Besmann [175]. From xray studies Besmann [175] found a single-phase $\mathrm{BC}_{0.43} \mathrm{~N}_{0.29}$ which he proposed was due to the substitution of carbon/nitrogen in the hexagonal BN structure. In addition, electron microprobe analysis revealed a carbon-rich phase $\mathrm{BC}_{1.02} \mathrm{~N}_{0.32}$ which was reported to have less crystallinity. The hardness of these single-phase coatings was found to be $4.2 \mathrm{GPa}$. The coefficient of friction of these coatings against alumina was found to be 0.39 . The details of the test conditions were not given.

There are no reports on amorphous BCN coatings. However, Devkon et al. [176] studied the tribological properties of nitrogen-implanted $\mathrm{B}_{4} \mathrm{C}$ at a contact stress of $0.003 \mathrm{GPa}$ to $1 \mathrm{GPa}$, with a $\mathrm{B}_{4} \mathrm{C}$ slider at room temperature. The coefficient of friction was observed to be less than 0.25 for both implanted and unimplanted samples. Their results indicated an increase in wear resistance and an increase in friction. Dekoven et al. did not perform hardness measurements but postulated an increase in hardness after implantation. Nastasi et al. [120] investigated the friction and wear properties of nitrogen-implanted $\mathrm{B}_{4} \mathrm{C}$ with a chromium steel slider. They 
found a reduction in both the friction coefficient and wear. They argued that nitrogen implantation into $\mathrm{B}_{4} \mathrm{C}$ will thermodynamically favor the precipitation of $\mathrm{BN}$ and carbon, which can act as a solid lubricant that can reduce friction. The literature on investigations of directly synthesized amorphous $\mathrm{BCN}$ coatings is limited.

\subsection{Objective}

This study explored whether metastable boron-carbon-nitrogen (BCN) coatings synthesized by DC magnetron sputtering would show any technologically useful tribological properties. Thus, metastable BCN coatings were characterized for mechanical and tribological properties for the first time. These preliminary tests were performed at room temperature and 50\% relative humidity under one load with ruby as a slider.

\subsection{Synthesis of amorphous BCN Coatings}

Amorphous boron-carbon-nitrogen coatings were prepared by using DC magnetron sputter deposition. Base pressure in the chamber was below $2 \times 10^{-8}$ Torr. A mixture of argon and nitrogen gas at $3 \mathrm{mTorr}$ pressure was used to sputter the target materials of $\mathrm{B}_{4} \mathrm{C}$ at a power of $500 \mathrm{~W}$ with no bias. By changing the percent of nitrogen in the sputtering gas, coatings with different compositions of boron, nitrogen, and carbon were prepared. The temperature of the substrate during deposition was less than $120^{\circ} \mathrm{C}$. Deposition rates were about $0.2 \mathrm{~nm} / \mathrm{s}$. Coatings were deposited on $380 \mu \mathrm{m}$ thick Si(100) substrates. Film thickness was controlled to be $1 \mu \mathrm{m}$.

Thickness of the coatings was measured by profilometry and found to deviate less than $3 \%$.

\subsection{Results}

The results of the investigation of $\mathrm{BCN}$ coatings are presented in the following sequence.

1) Composition and microstructure analysis, 
2) Mechanical properties,

3) Tribological properties, and

4) Discussion of the results.

\subsubsection{Composition and Microstructure Analysis}

In addition to silicon substrates, the depositions were also done on $\mathrm{Nb}$-coated carbon substrates used for ion beam compositional analysis. $\mathrm{Nb}$ was used to shift the carbon, boron, and nitrogen peaks away from the substrate signal. A $2 \mathrm{MeV} 4 \mathrm{He}^{+}$ beam was used to perform RBS on these samples. The RUMP-code [125] was used to simulate the experimental spectra.

The RBS spectra of Sample 4 along with the simulation are presented in Figure 7.1a. The region of the lighter elements is expanded and is shown in Figure 7.1b. Figure 7.2 shows the RBS spectra of the coatings with and without nitrogen. The difference in composition profiles of nitrogen, carbon, and boron are shown in this figure. The coating compositions extrapolated from the simulated spectra are presented in a ternary diagram, which is shown in Figure 7.3. This data showed that the composition varied along the composition line between $\mathrm{B}_{4} \mathrm{C}$ and $\mathrm{N}$. Coating compositions are also summarized in Table 7.1.

Table 7.1 Composition of BCN coatings.

\begin{tabular}{|c|c|c|c|}
\hline Sample & $\begin{array}{c}\text { Boron } \\
\text { at.\% }\end{array}$ & $\begin{array}{c}\text { Carbon } \\
\text { at.\% }\end{array}$ & $\begin{array}{c}\text { Nitrogen } \\
\text { at.\% }\end{array}$ \\
\hline 1 & 73.2 & 17.9 & 8.9 \\
\hline 2 & 58.0 & 17.1 & 24.9 \\
\hline 3 & 39.8 & 15.6 & 44.6 \\
\hline 4 & 38.4 & 13.5 & 48.1 \\
\hline 5 & 82.1 & 17.9 & 0.00 \\
\hline
\end{tabular}

The diffraction pattern of these coatings obtained from TEM studies is shown in Figure 7.4. The diffraction patterns had diffuse rings, indicating the amorphous nature of the $\mathrm{BCN}$ coatings. Films synthesized with a $\mathrm{B}_{4} \mathrm{C}$ composition also showed the presence of an amorphous structure. 
Residual stresses in these coatings were measured by a curvature method as discussed in Chapter 3. The coatings had intrinsic compressive stress of about $500 \mathrm{MPa}$. Intrinsic stress in the coatings did not change with nitrogen content in the coatings. 


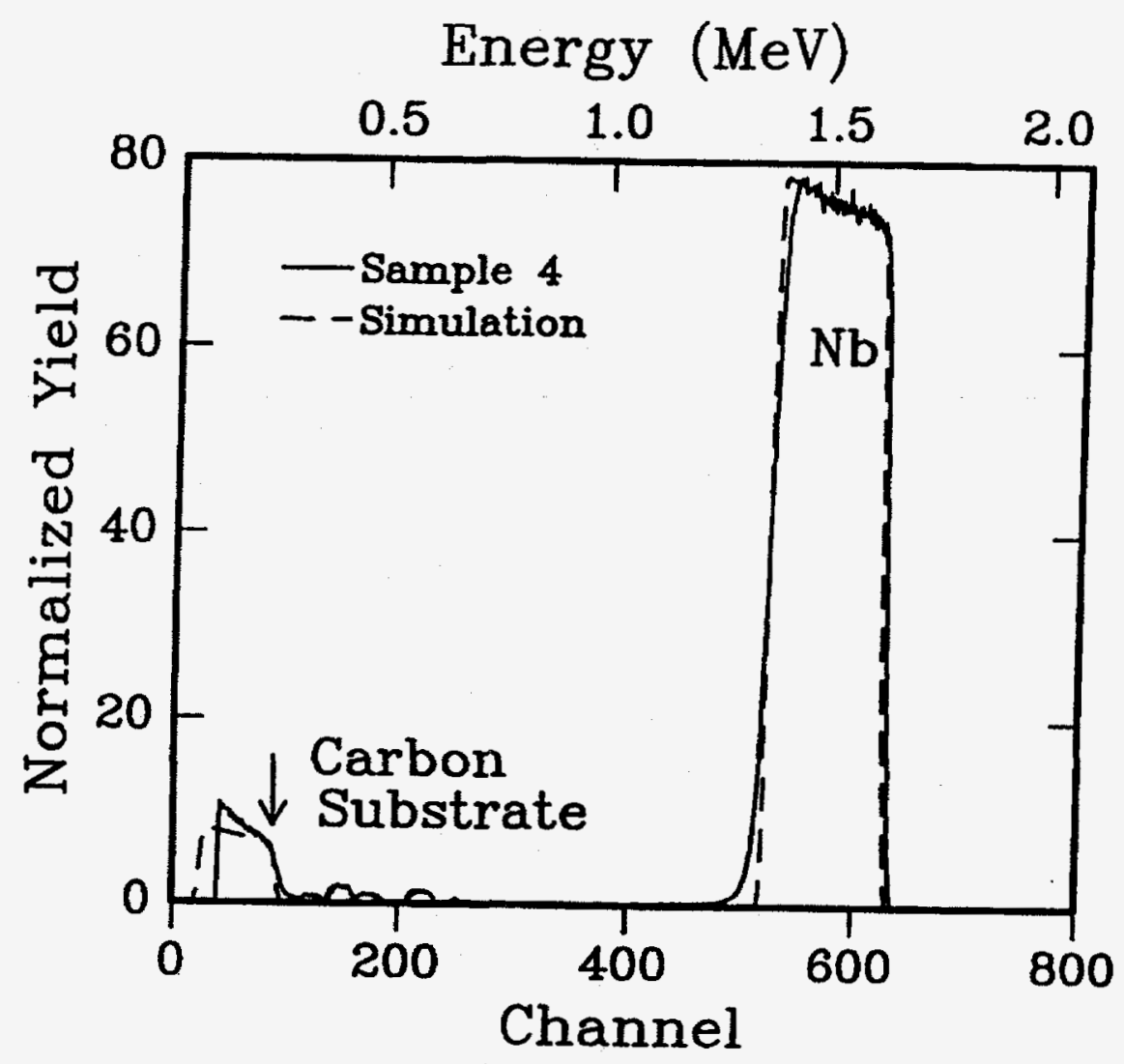

Figure 7.1a Rutherford backscattering spectra of the sample $\mathrm{BCN}$ coating along with simulated spectra for Sample 4. 


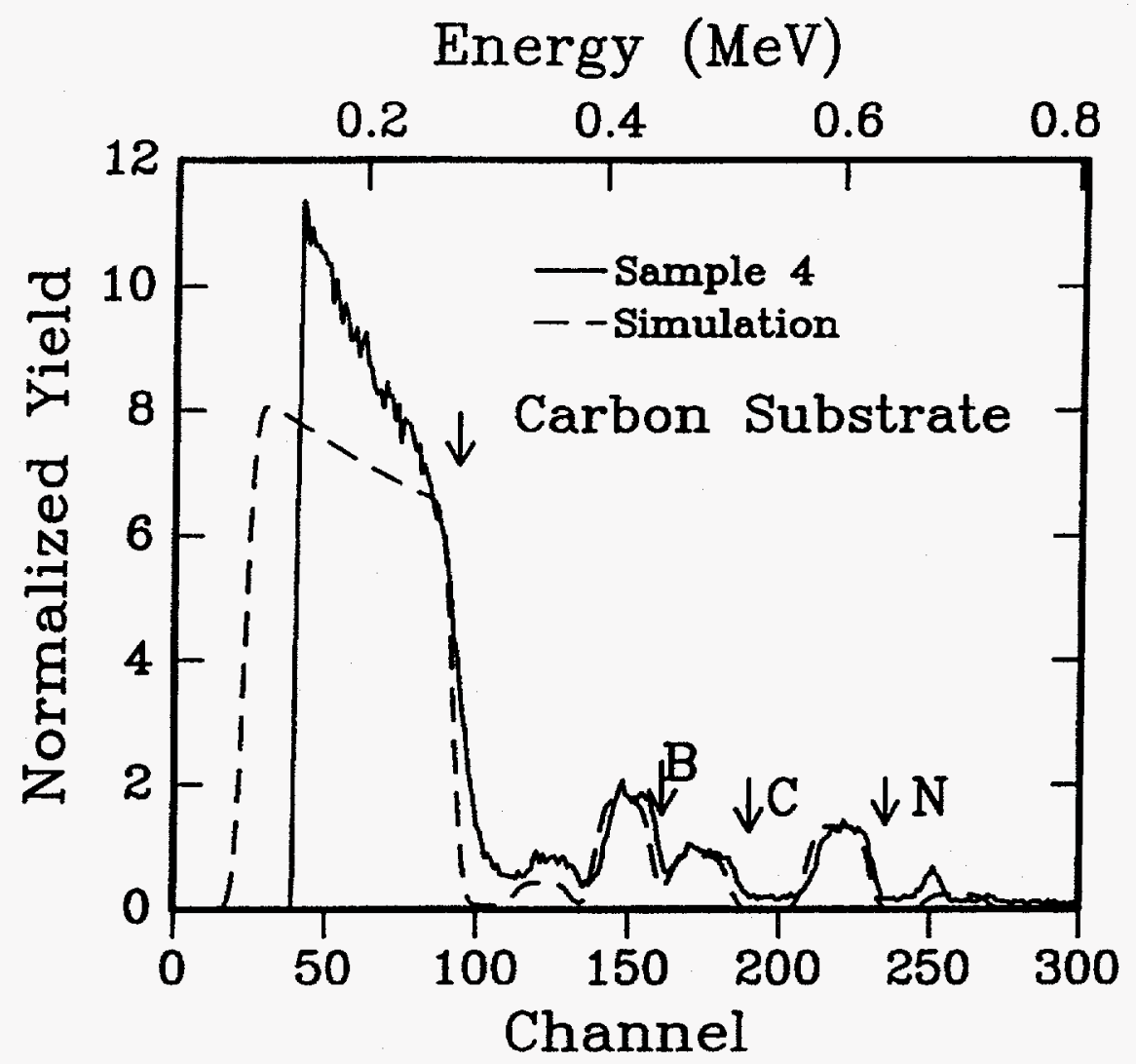

Figure 7.1b Rutherford backscattering spectra of the sample BCN coating Sample 4 (expanded to show the lighter elements). 


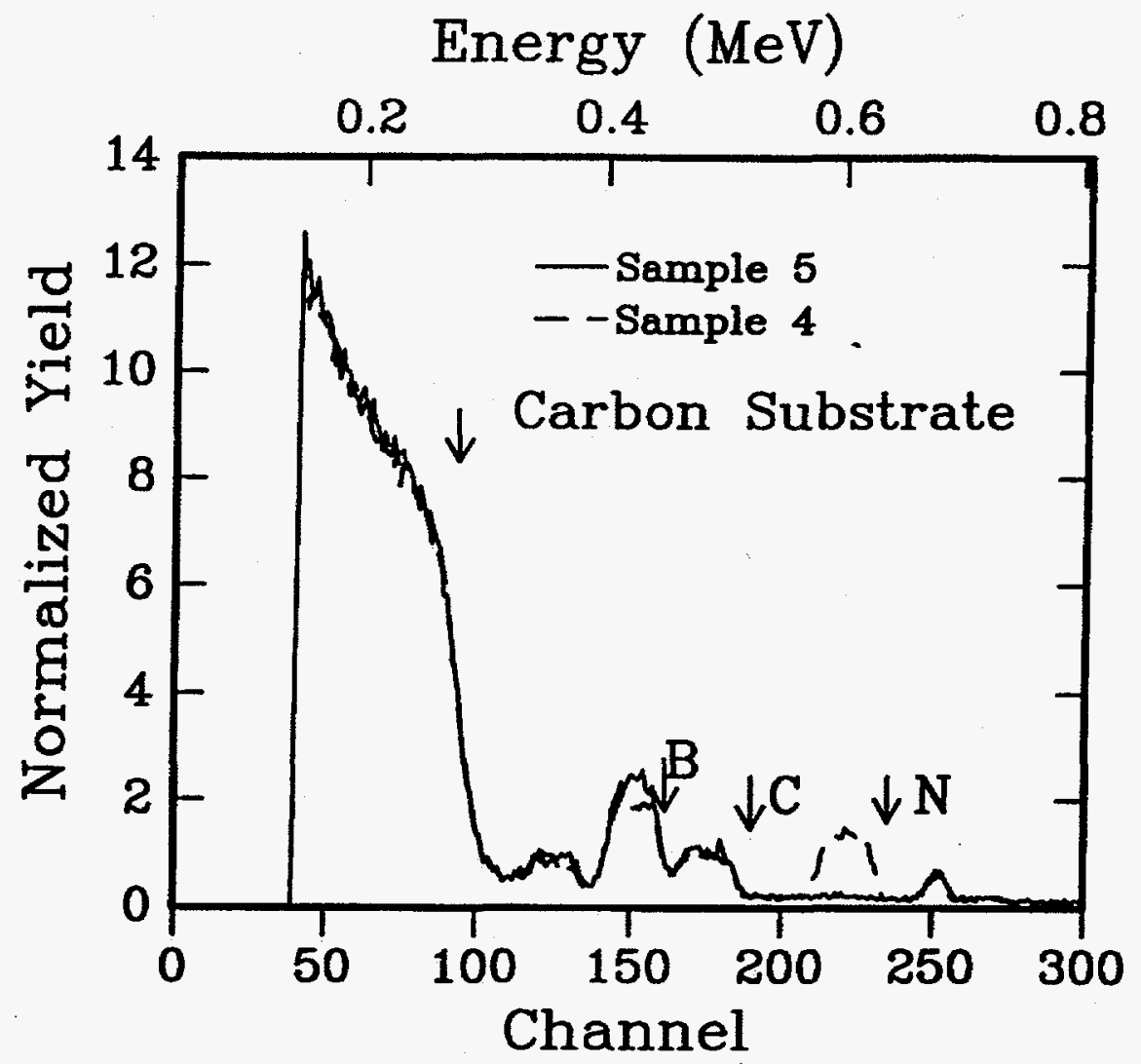

Figure 7.2 Rutherford backscattering spectra of the BCN coatings (Samples 4 and 5). 


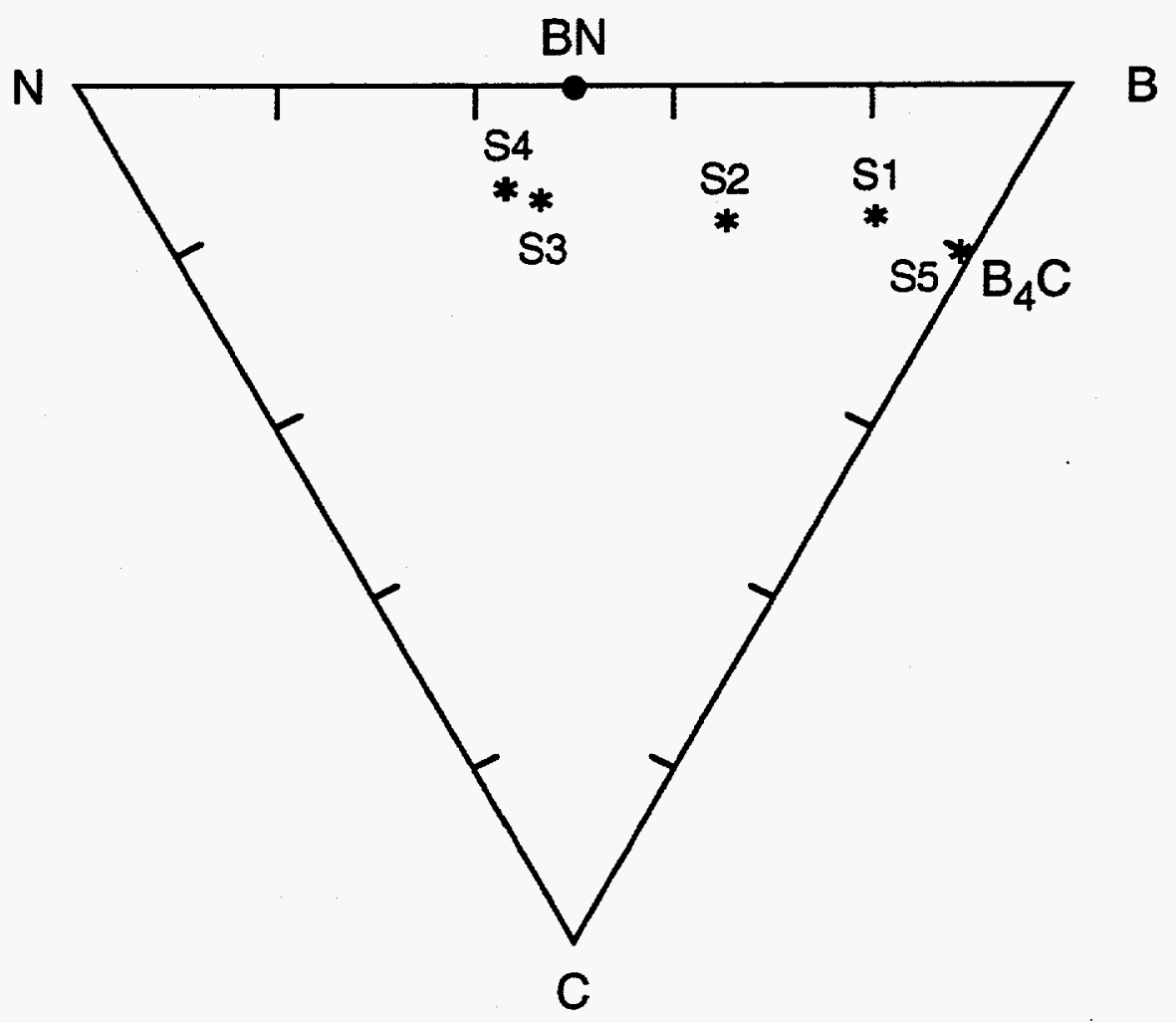

Figure 7.3. Composition triangle of the $\mathrm{BCN}$ coatings. 


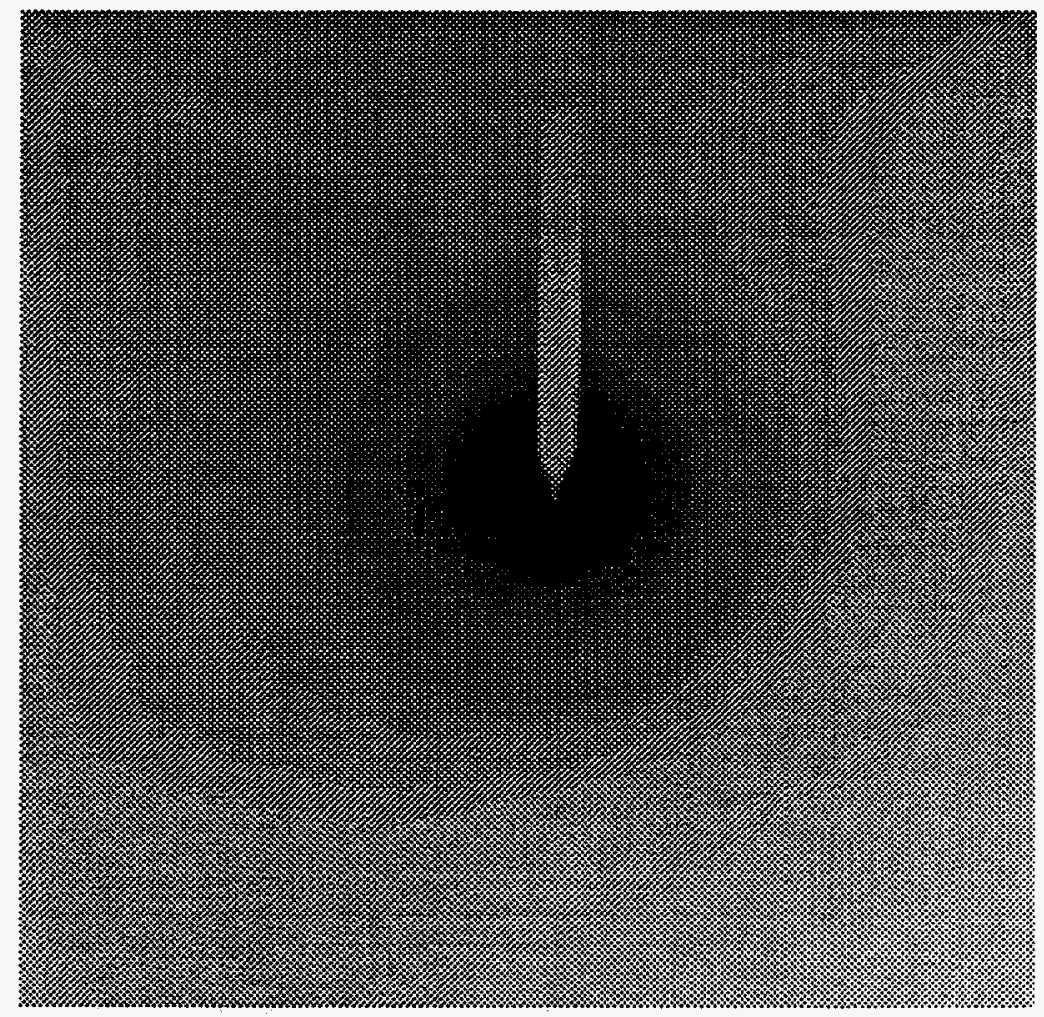

Figure 7.4. Selected area diffraction pattern of the BCN coatings. 


\subsubsection{Mechanical Properties}

Surface hardness measured using a nano-indenter as described in Chapter 3 and apparent fracture toughness of the coating-substrate system using a microindenter are discussed in the following sections.

\subsubsection{Surface Hardness}

The nanohardness measured in these coatings at a depth of $47 \mathrm{~nm}$ is presented in Figure 7.5. To minimize the substrate effects, the penetration depth at which the hardness was taken was two orders of magnitude less than the coating thickness. The data showed that the hardness of these BCN coatings decreased with an increase in nitrogen content. A summary of elastic moduli and hardness is given in Table 7.2.

\subsubsection{Apparent Fracture Toughness}

A microindenter with loads ranging from 25 to 1000 grams was used to determine the cracking response of $\mathrm{BCN}$ coatings on silicon substrates. Figure 7.6 shows the crack length dependence on load for the uncoated silicon and sample S1. The length of the radial cracks generated at the corners of an indent were shorter in the coatings compared to the bare silicon. Below 100 grams, no radial cracks were observed for $\mathrm{BCN}$ coatings in contrast to uncoated silicon. At 200 grams, the observed radial cracks did not meet the criteria, $C \geq 2 a$, where $C$ is the crack length and $2 \mathrm{a}$ is the diagonal length of the indent. Thus, only well-defined cracks are included in Figure 7.6. The depth of an indent was greater than the coating thickness at all loads. For example, the depth of an indent for Sample 1 at a load of 500 grams was $6.1 \mu \mathrm{m}$, which was greater than the coating thickness. The apparent fracture toughness of the coatings, calculated from Figure 7.6 (crack length, $\mathrm{C}^{3 / 2}$, 
Table 7.2 Summary of hardness and elastic modulus of BCN coatings.

\begin{tabular}{|c|c|c|c|c|c|}
\hline Sample & $\begin{array}{c}\text { Boron } \\
\text { at. } \%\end{array}$ & $\begin{array}{c}\text { Carbon } \\
\text { at.\% }\end{array}$ & $\begin{array}{c}\text { Nitrogen } \\
\text { at.\% }\end{array}$ & $\begin{array}{c}\text { Hardness } \\
(\mathrm{GPa})\end{array}$ & $\begin{array}{c}\text { Elastic Modulus* } \\
(\mathrm{GPa})\end{array}$ \\
\hline 1 & 73.2 & 17.9 & 8.9 & $34.9 \pm 1.4$ & $334.9 \pm 12.0$ \\
\hline 2 & 58.0 & 17.1 & 24.9 & $25.9 \pm 1.0$ & $264.9 \pm 9.0$ \\
\hline 3 & 39.8 & 15.6 & 44.6 & $20.9 \pm 1.0$ & $201.0 \pm 7.0$ \\
\hline 4 & 38.4 & 13.5 & 48.1 & $20.2 \pm 0.8$ & $194.0 \pm 6.0$ \\
\hline 5 & 82.1 & 17.9 & 0.00 & $41.5 \pm 1.5$ & $367.0 \pm 11.0$ \\
\hline Silicon & & & & $16.07 \pm 0.7$ & $191.01 \pm 9.0$ \\
\hline * Measurements taken at a depth of 47 nm & & & & \\
\hline
\end{tabular}




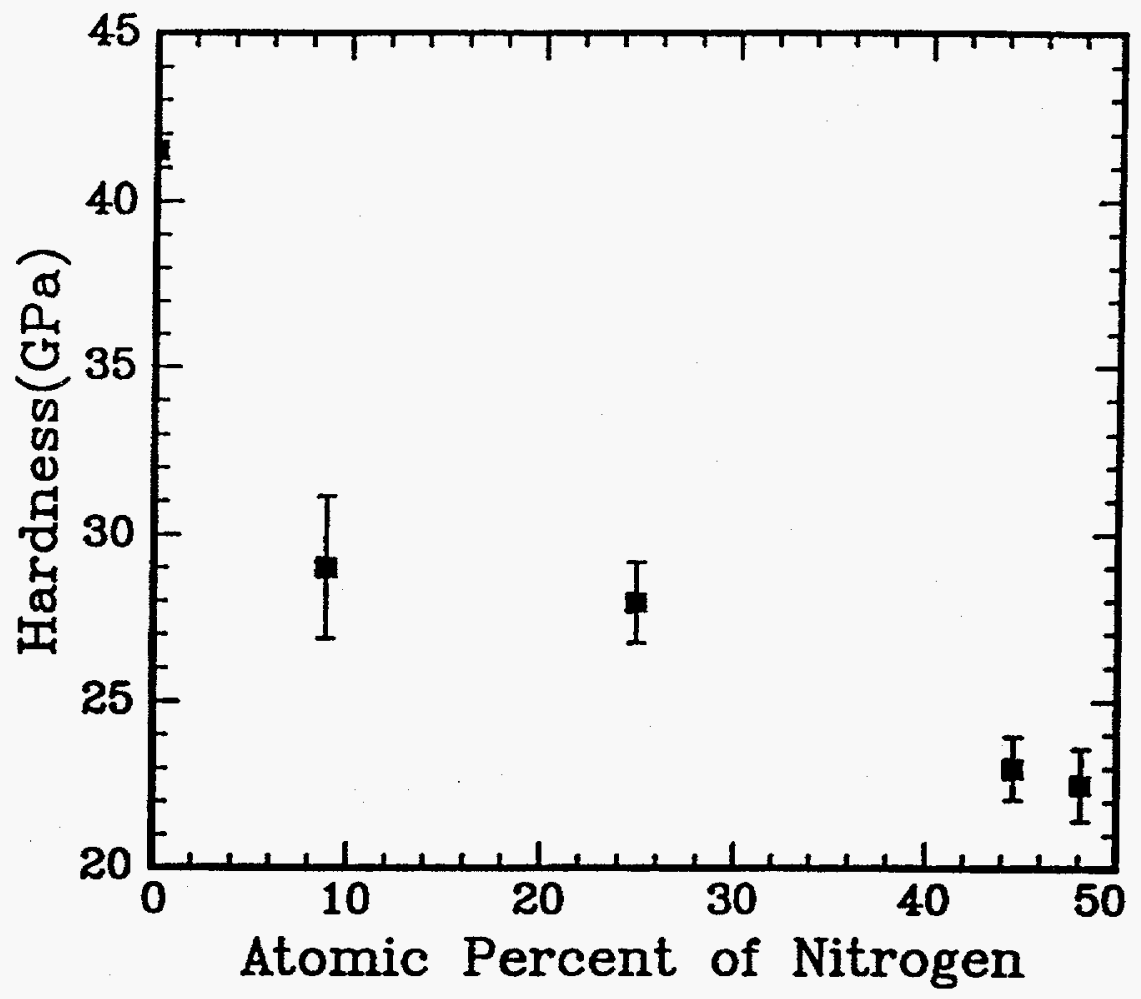

Figure 7.5 Surface hardness at a depth of $47 \mathrm{~nm}$ as a function of at.\% of nitrogen. 
Table 7.3 Summary of the apparent fracture toughness of BCN coatings.

\begin{tabular}{|c|c|c|c|c|}
\hline Sample & $\begin{array}{c}\text { Boron } \\
\text { at.\% }\end{array}$ & $\begin{array}{c}\text { Carbon } \\
\text { at.\% }\end{array}$ & $\begin{array}{c}\text { Nitrogen } \\
\text { at.\% }\end{array}$ & $\begin{array}{c}\text { Fracture Toughness } \\
(\text { MPa.m }\end{array}$ \\
\hline 1 & 73.2 & 17.9 & 8.9 & $1.0 \pm 0.01$ \\
\hline 2 & 58.0 & 17.1 & 24.9 & $0.95 \pm 0.04$ \\
\hline 3 & 39.8 & 15.6 & 44.6 & $0.86 \pm 0.06$ \\
\hline 4 & 38.4 & 13.5 & 48.1 & -- \\
\hline 5 & 82.1 & 17.9 & 0.00 & $1.05 \pm 0.06$ \\
\hline Silicon & & & & $0.67 \pm 0.02$ \\
\hline
\end{tabular}


versus the load) and from Eqn. [3.15], is summarized in Table 7.3. The data indicated that all five samples had higher apparent fracture toughness than the uncoated silicon substrate.

\subsubsection{Tribological Properties}

Tribological studies were performed using a POD apparatus under a normal load of $0.8 \mathrm{~N}$ and at $50 \%$ relative humidity, with a ruby pin as a slider. Sliding speed of $68.4 \mathrm{~mm} / \mathrm{sec}$ was maintained during the friction and wear tests. The static contact stress for uncoated silicon was 0.6 GPa. Depending on the coating modulus, the contact stress for $\mathrm{BCN}$ coatings varied from $0.83 \mathrm{GPa}$ to $1 \mathrm{GPa}$. The friction coefficient of Samples 1, 5, and uncoated silicon are shown in Figure 7.7. The average friction coefficient of the coatings varied from 0.5 to 0.7 . Sample 5 had a steady friction. There were large fluctuations in the friction coefficient of uncoated silicon and $\mathrm{BCN}$ coatings.

The wear tracks of the uncoated sample, and samples 1 and 5 that were investigated using scanning electron microscopy are shown in Figures 7.8 and 7.9. Figure 7.10 shows the wear track profiles measured by profilometry. The depth of wear tracks was less than the coating thickness for all the measurements. The depth of the wear tracks in the uncoated silicon and BCN coatings were $6 \mu \mathrm{m}$ and $0.2 \mu \mathrm{m}$, respectively. Depth of the wear track of Sample 2 was approximately $0.6 \mu \mathrm{m}$, which is still less than the coating thickness. The wear area determined from these wear profiles was used to calculate the wear factor using Eqn.[3.14]. 


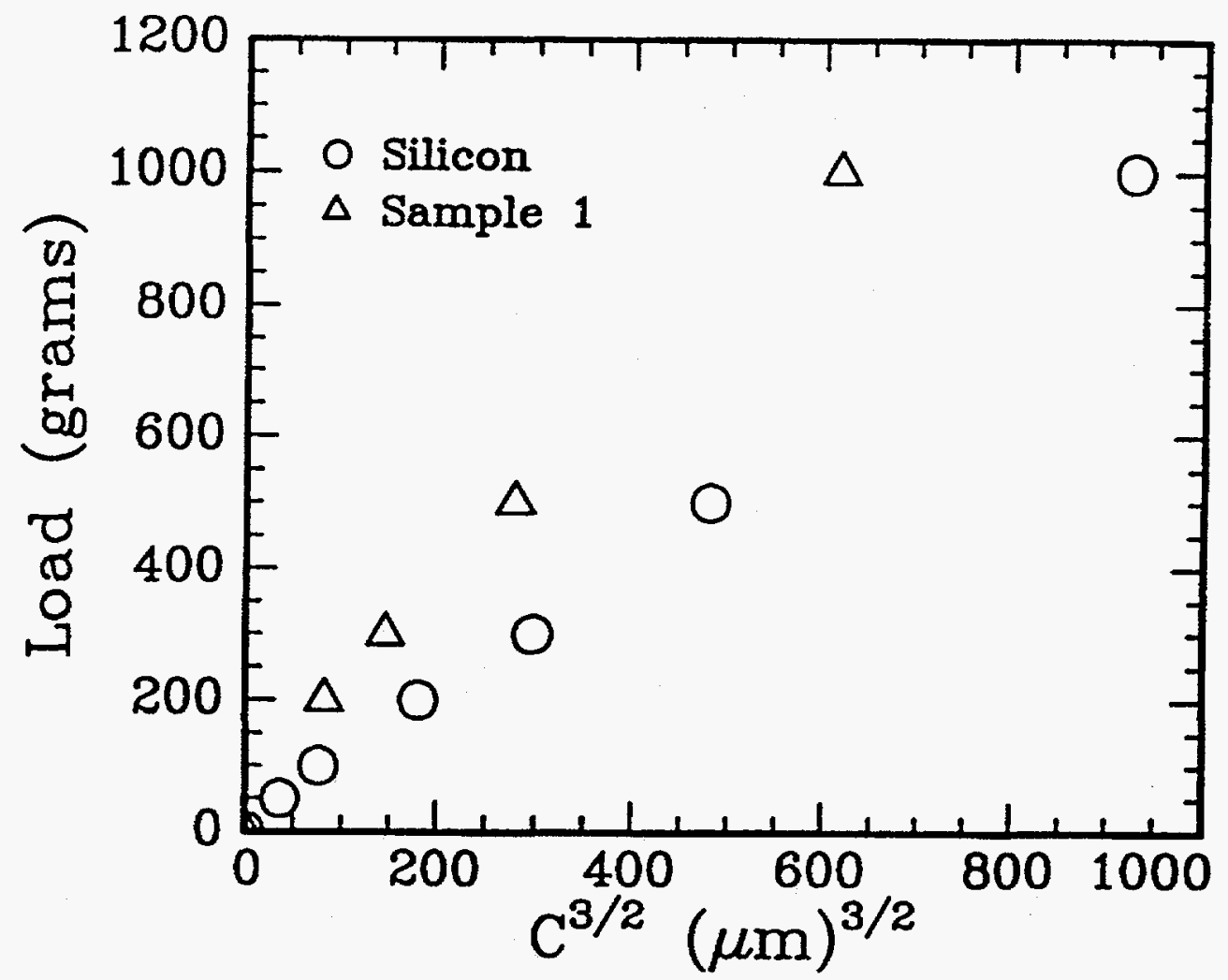

Figure 7.6 Results from the data of well-defined radial cracks as a function of an applied load.(a) Uncoated silicon and Sample 1. 


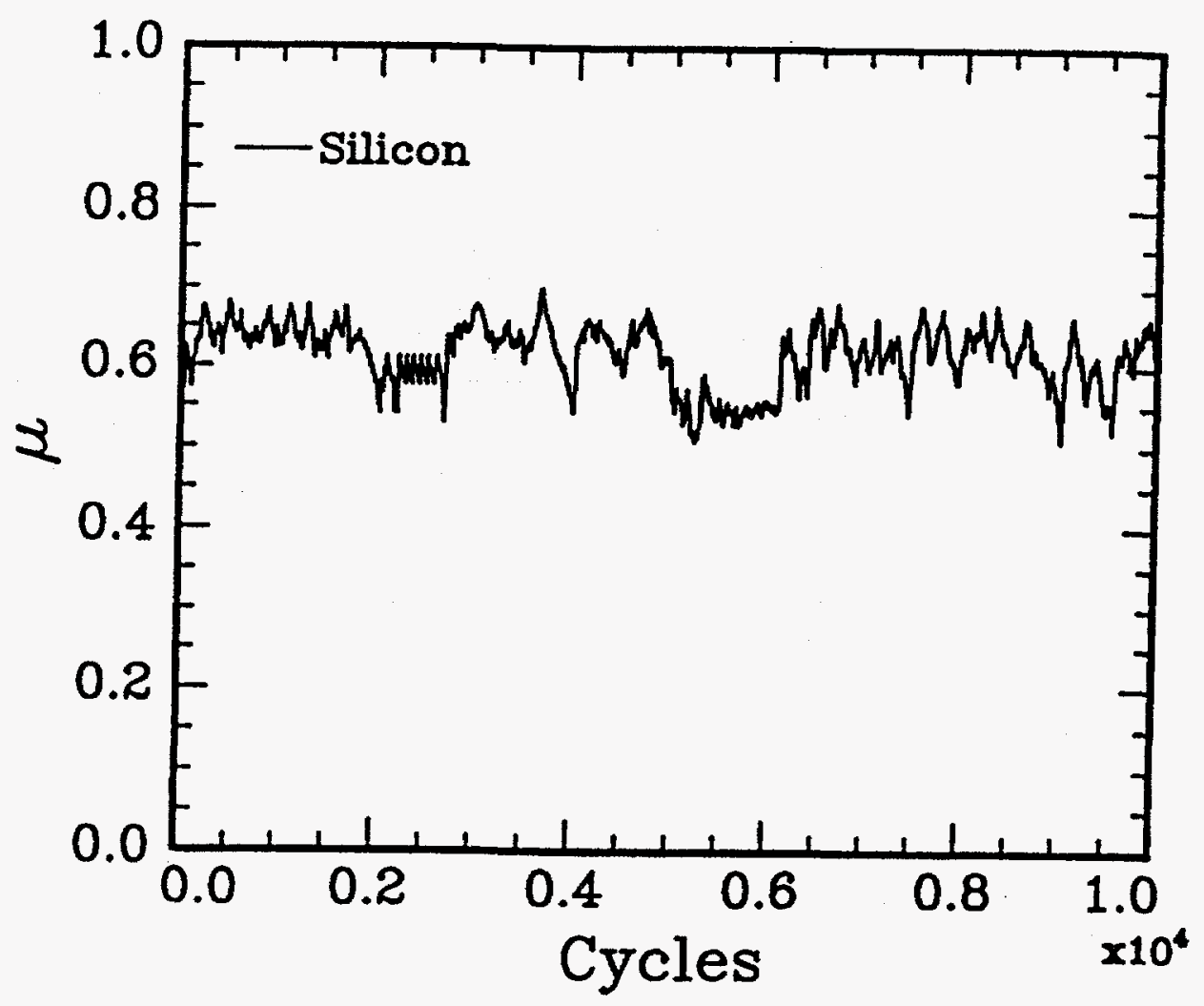

Figure 7.7 Coefficient of friction. (a) Uncoated silicon at $0.8 \mathrm{~N}$ with ruby pin. 


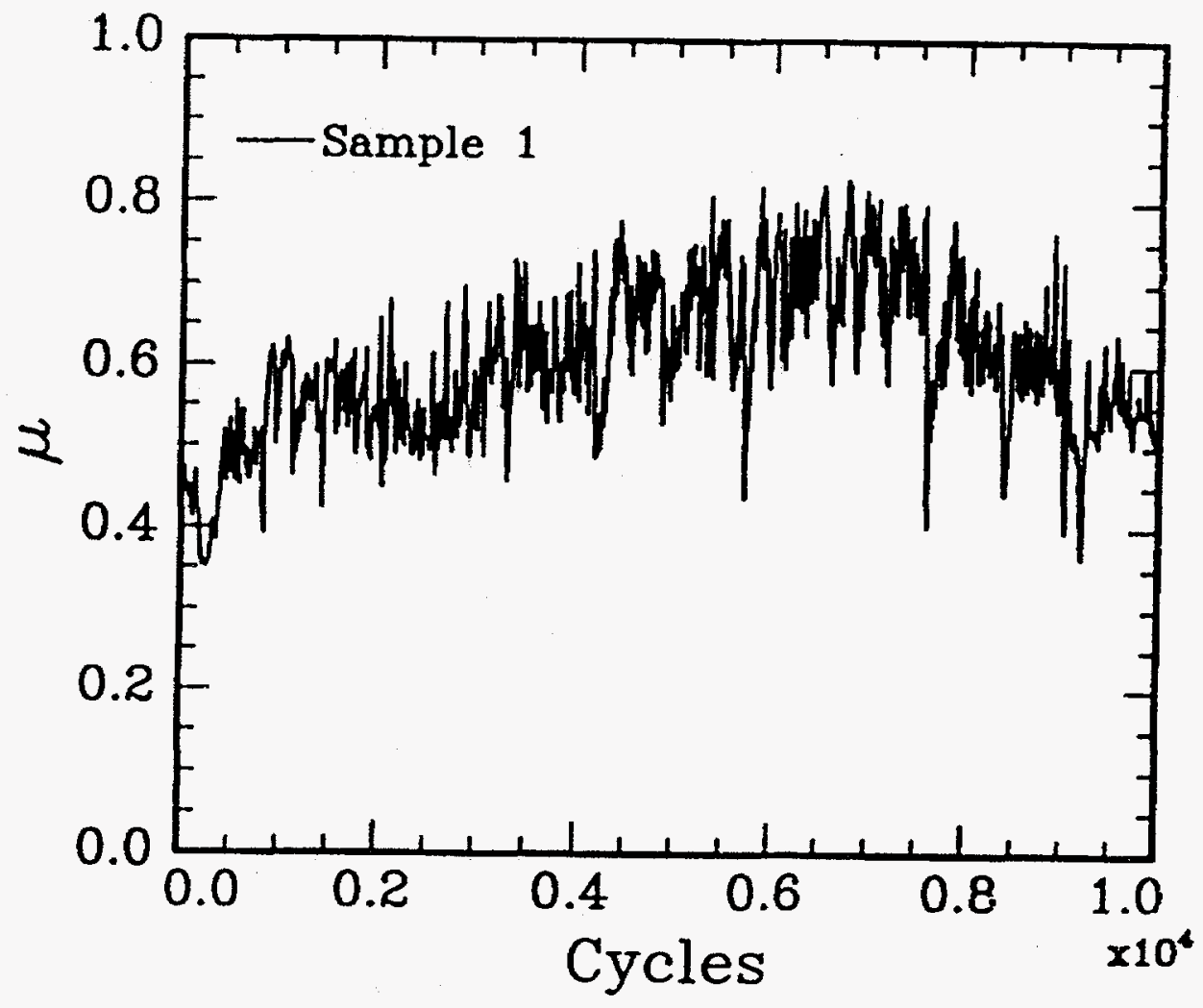

Figure 7.7 Coefficient of friction. (b) Sample 1 at $0.8 \mathrm{~N}$ with ruby pin. 


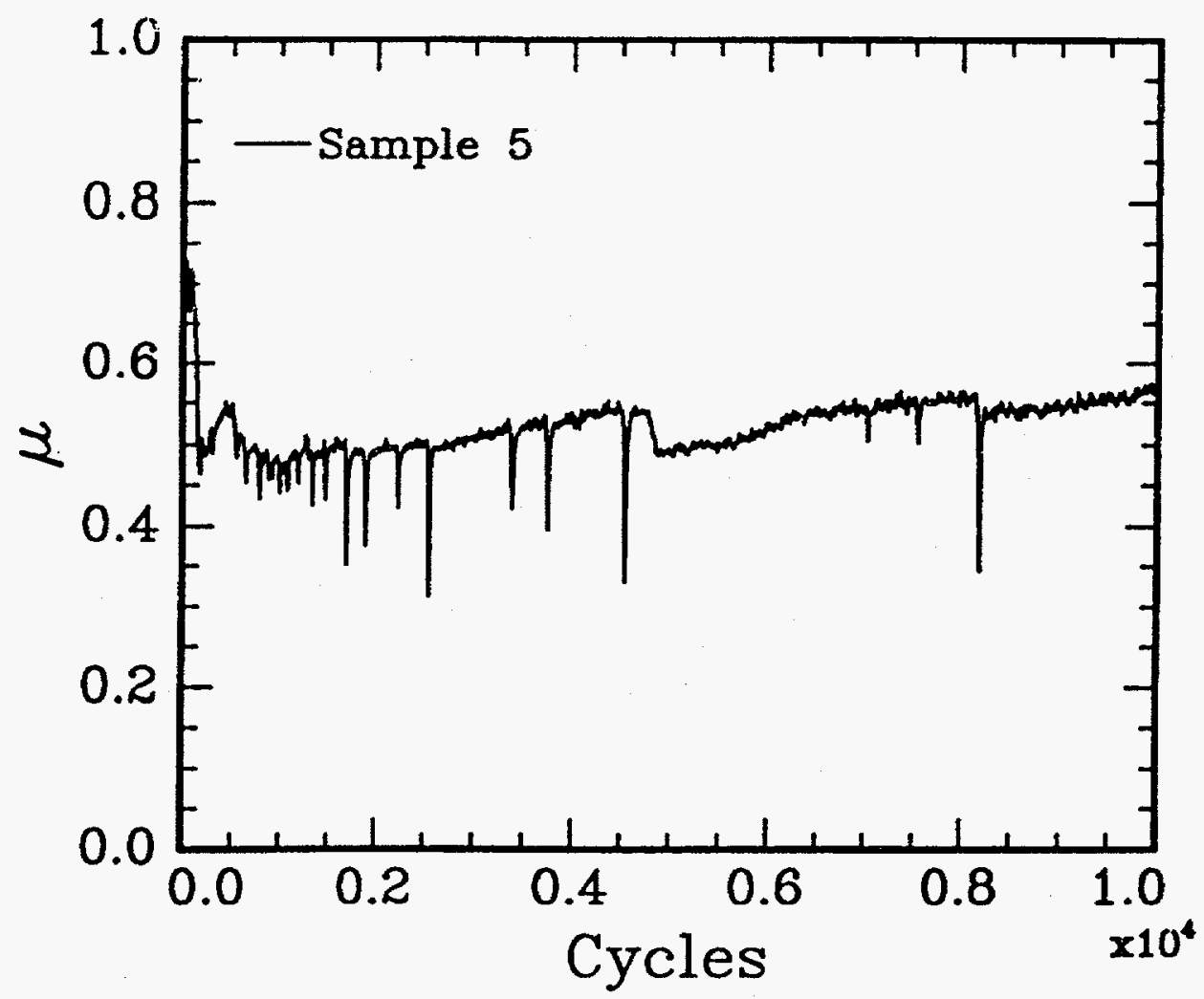

Figure 7.7 Coefficient of friction. (c) Sample 5 at $0.8 \mathrm{~N}$ with ruby pin. 


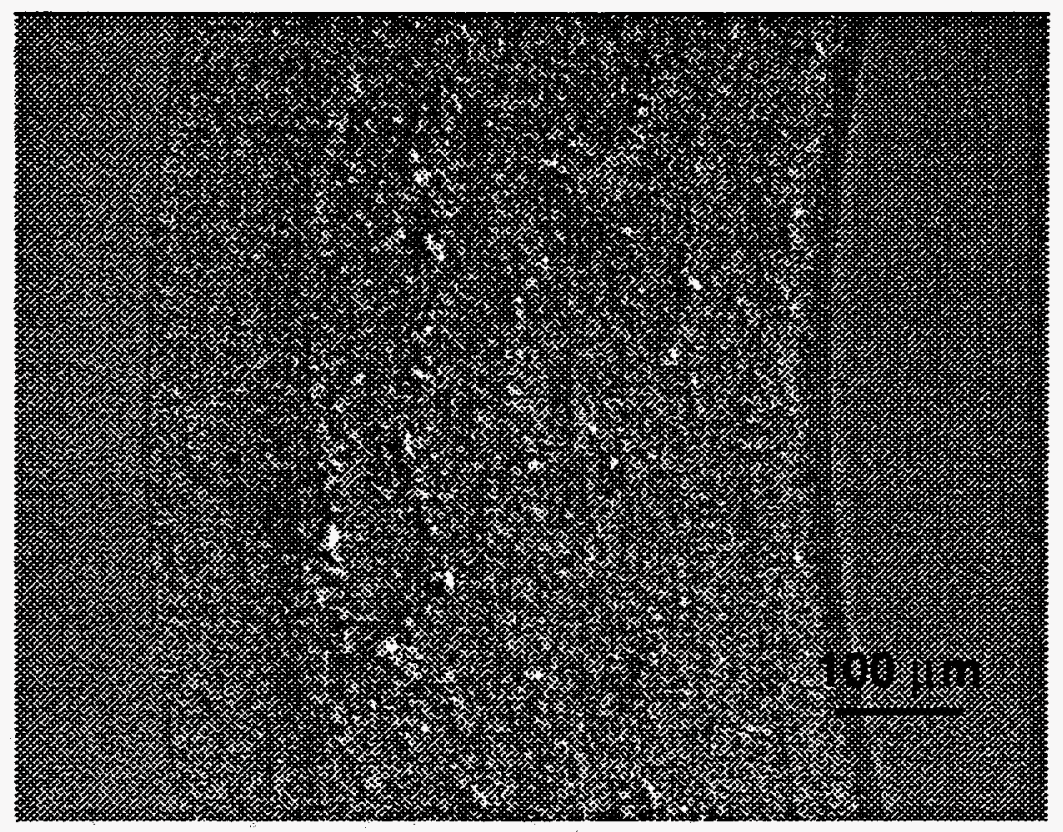

Figure 7.8. Scanning electron micrograph of the wear track of the uncoated silicon. 


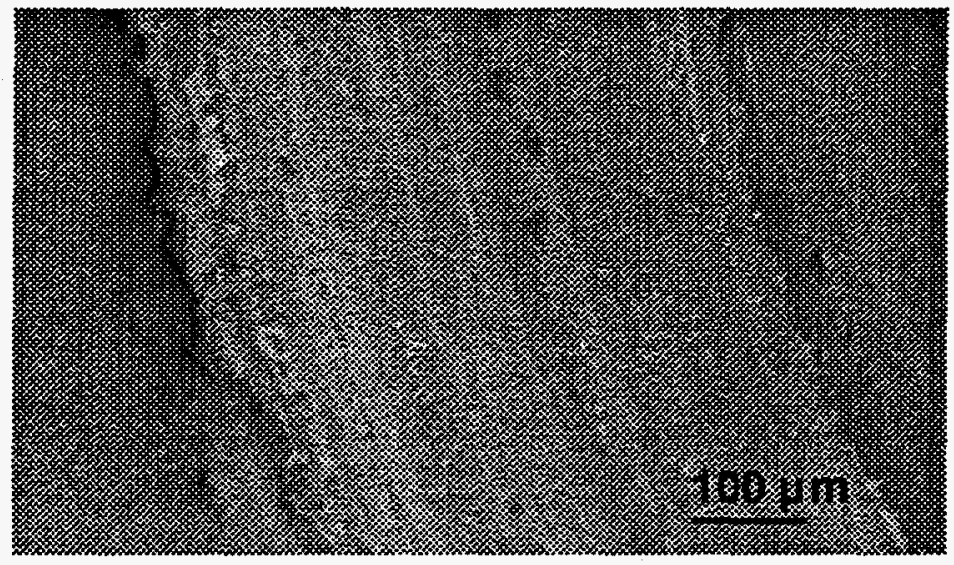

(a)

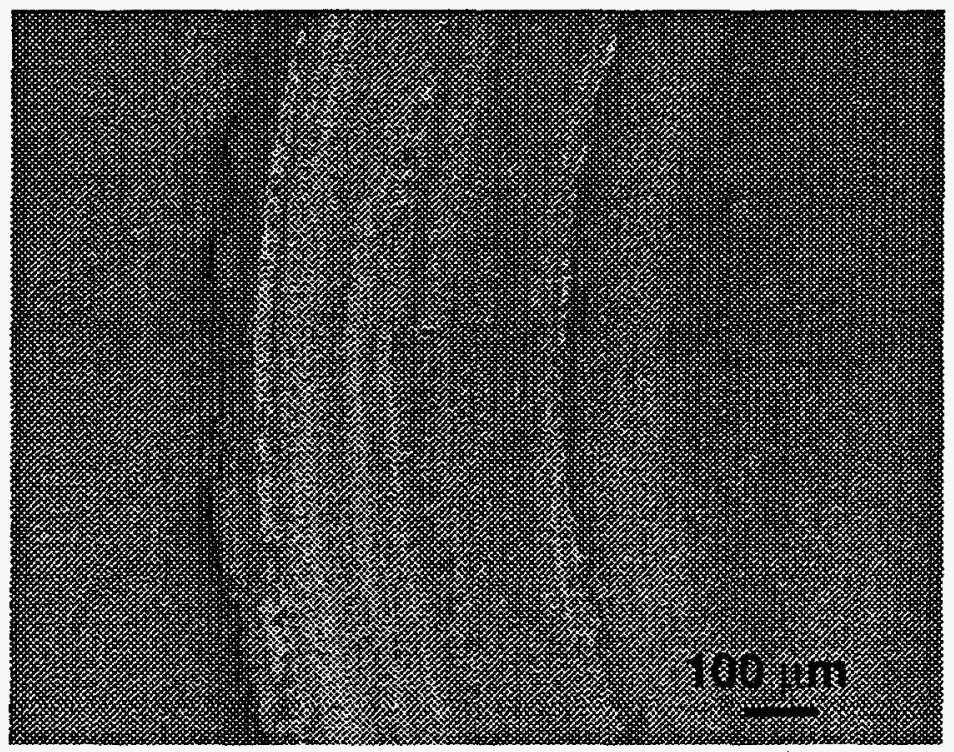

(b)

Figure 7.9. Scanning electron micrographs showing the wear morphology of (a) Sample 1, (b) Sample 5. 


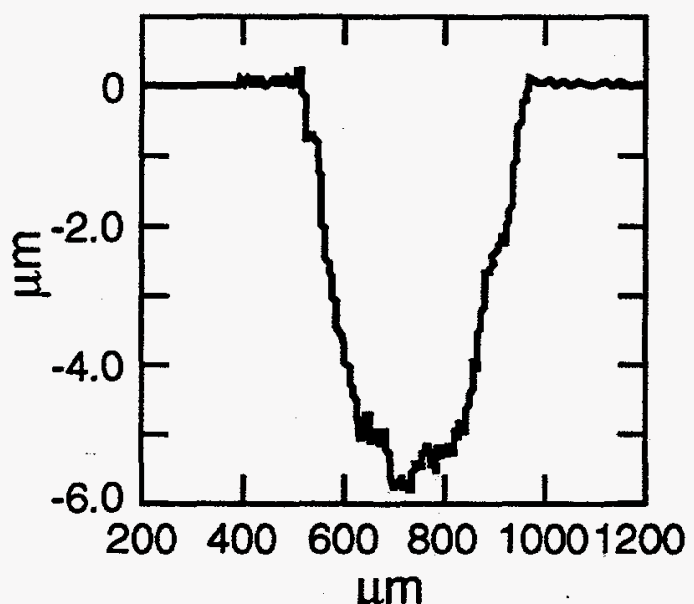

(a)

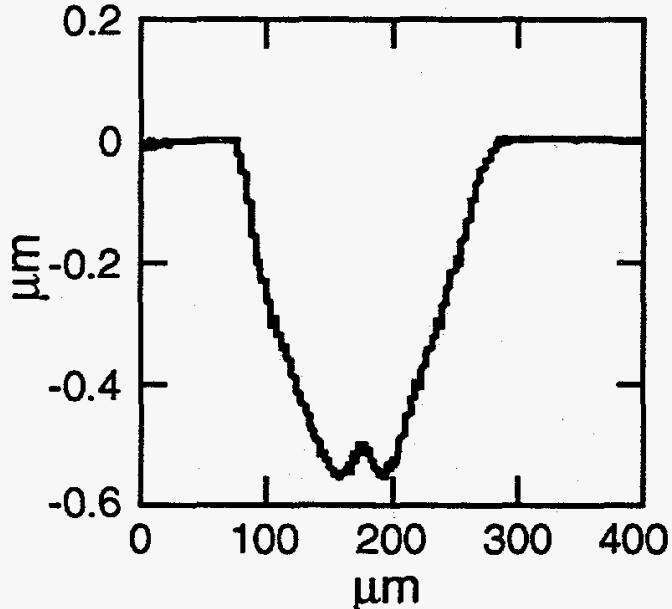

(c)

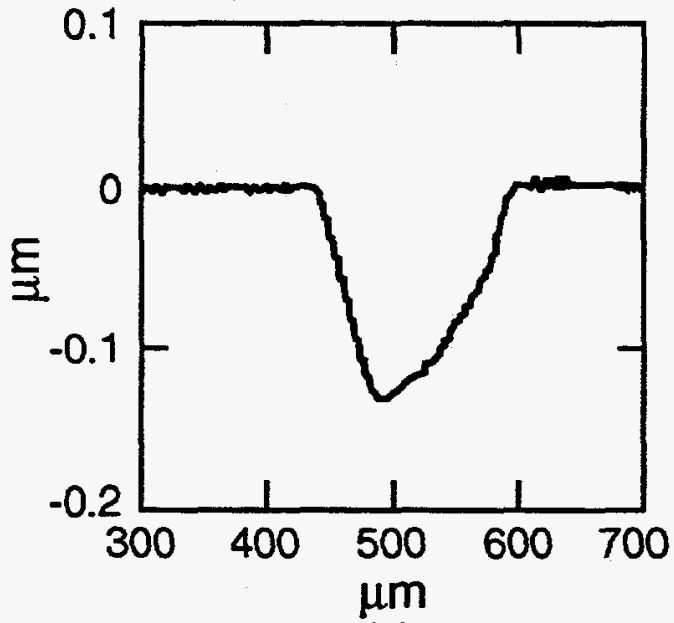

(e)

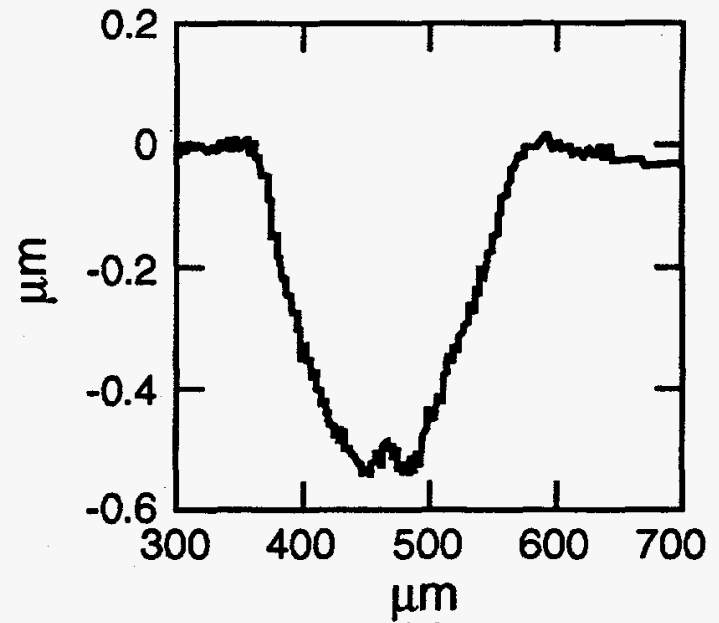

(b)

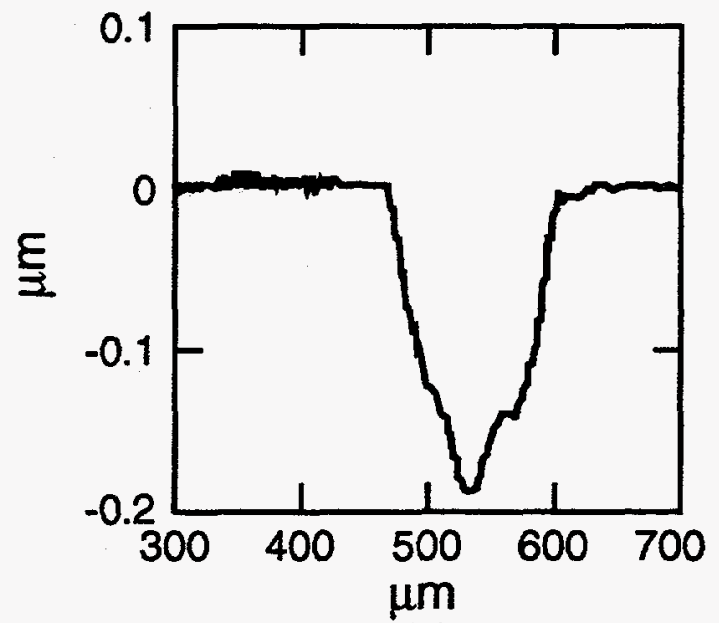

(d)

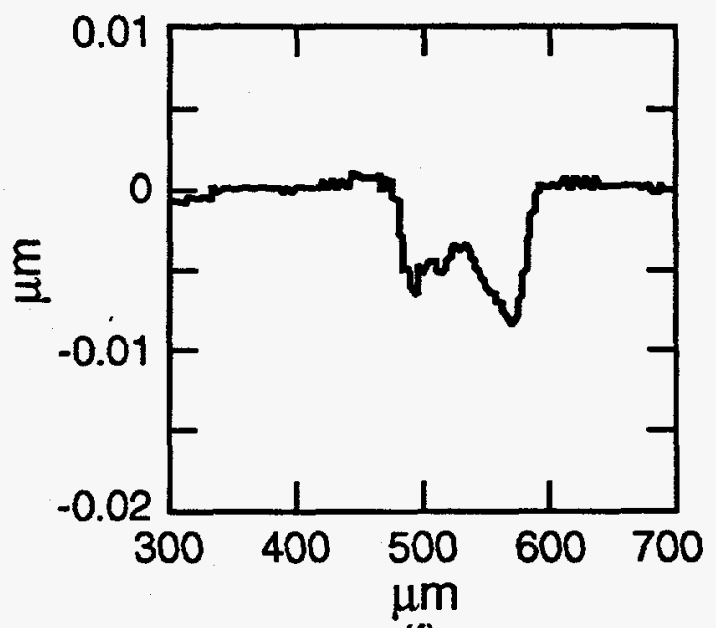

(f)

Figure 7.10. Wear track profiles for 10000 cycles,

(a) Uncoated silicon, (b) Sample 1, (c) Sample 2,

(d) Sample 3, (e) Sample 4, (f) Sample 5. 
The trends in the wear factor as a function of hardness are presented in the Figure 7.11. In this plot, a simplified notation $\mathrm{S} 1$ is used for Sample 1. The wear factor values are summarized in Table 7.4. Dispite its high hardness and high toughness, coating $\mathrm{S} 1$ showed a high wear factor.

Table 7.4 Summary of wear factor and the apparent fracture toughness of $\mathrm{BCN}$ coatings.

\begin{tabular}{|c|c|c|}
\hline Sample & $\begin{array}{c}\text { Fracture Toughness } \\
\left(\mathrm{MPa} . \mathrm{m}^{1 / 2}\right)\end{array}$ & $\begin{array}{c}\text { Wear Factor } \\
\left(10^{-6} \mathrm{~mm}^{3} / \mathrm{N} . \mathrm{m}\right)\end{array}$ \\
\hline 1 & $1.0 \pm 0.01$ & $8.8 \pm 0.18$ \\
\hline 2 & $0.95 \pm 0.04$ & $3.6 \pm 0.25$ \\
\hline 3 & $0.86 \pm 0.06$ & $2.3 \pm 0.42$ \\
\hline 4 & -- & $1.5 \pm 0.123$ \\
\hline 5 & $1.05 \pm 0.06$ & $0.82 \pm 0.02$ \\
\hline Silicon & $0.67 \pm 0.02$ & $380 \pm 30$ \\
\hline
\end{tabular}

\subsection{Discussion of the Results}

The investigations of $\mathrm{BCN}$ coatings were intended to contribute to the development of new compound coatings. However, the results from this research seems to be very complicated. In addition, the data was very limited. Thus, the experimental data was not sufficient for a complete the analysis of the tribological and mechanical behavior of these coatings. More experiments need to be carried out in order to isolate and identify the wear mechanisms against the ruby slider under the present testing conditions.

The nanohardness data indicated that an amorphous coating with a composition close to stochiometric $\mathrm{B}_{4} \mathrm{C}$ had a hardness of $41 \mathrm{GPa}$ and an elastic modulus of 367 $\mathrm{GPa}$. The reported values of hardness and modulus for a polycrystalline $\mathrm{B}_{4} \mathrm{C}$ prepared 


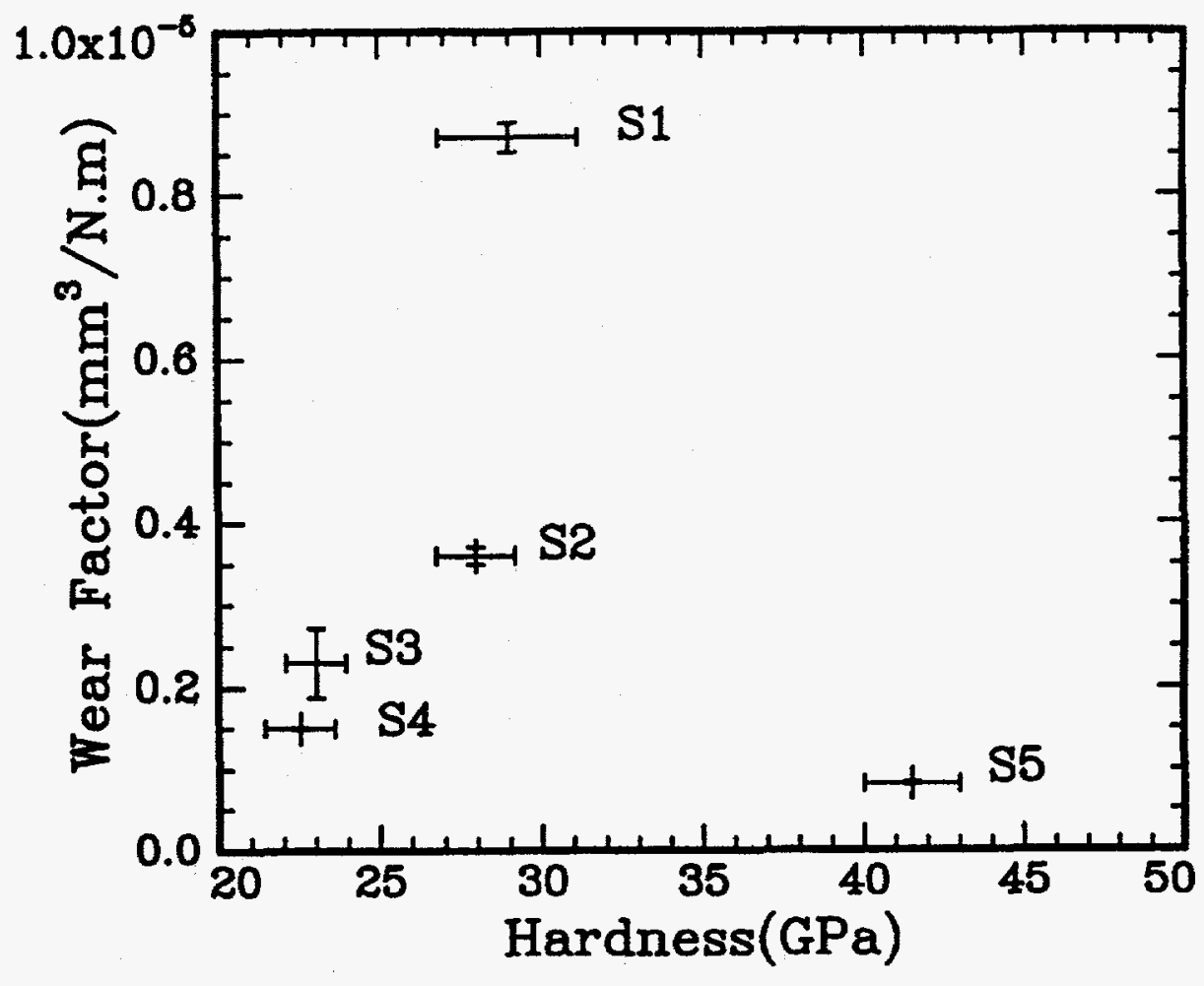

Figure 7.11 Wear factor as a function of hardness. 
by sintering were $44.15 \mathrm{GPa}$ and $460 \mathrm{GPa}$, respectively [54]. The data indicated that $\mathrm{B}_{4} \mathrm{C}$ coatings synthesized in this study had a hardness equal to polycrystalline $\mathrm{B}_{4} \mathrm{C}$, whereas the elastic modulus of the coating was $25 \%$ less than polycrystalline $\mathrm{B}_{4} \mathrm{C}$. Thus, the measured hardness for the amorphous boron carbide coating was in agreement with the value reported for polycrystalline $\mathrm{BCN}$ in the literature [54].

Samples 3 and 4 had approximately the same coating hardness and moduli, where the atomic percent (at.\%) of nitrogen in these coatings was greater than 40 . The hardnesses of these two coatings (samples 3 and 4) were half the hardness of the coating with no nitrogen (sample 5). The data also suggested that both pure amorphous boron carbide and coatings with 9 at.\% of nitrogen were hard.

The only studies that can be compared with these results are by Dekempeneer et al. [177]. Dekempeneer et al. synthesized amorphous BCN coatings by adding boron and /or nitrogen to hard DLC coatings. In that study, Dekempeneer et al. [142] observed that the hardness of DLC coatings decreased with the addition of boron and/or nitrogen. They reported the hardness for a pure DLC and for a coating with 42 at. $\%$ of nitrogen, $43 \%$ carbon, and $15 \%$ boron to be 20 and $9 \mathrm{GPa}$, respectively. The coatings in this investigation were harder than the carbon-rich $\mathrm{BCN}$ coatings synthesized by Dekempeneer et al [177].

The large amount of wear debris that was found in the wear track (Figures 7.8a-c) might have been responsible for the fluctuations in the friction coefficient. Fracture of the coatings might have caused the fluctuations in the friction coefficient. The hardness of $\mathrm{BCN}$ coatings was greater than the ruby pin ( $20 \mathrm{GPa}$ ). In the friction and wear tests of samples 1,2, and 5, wear of the pin might have been expected. In this study, the pin did not show any visible wear.

The SEM micrographs (Figure 7.9) of the wear tracks indicated parallel grooves running along the wear track, suggesting that abrasive wear was one of the dominant wear mechanism. Wear morphology observed for all the coatings was 
similar. The large amount of loose wear debris found in the wear track suggested that brittle fracture was another possible mechanism of wear.

Adhesion of the coating to the substrate was strong since delamination of the coatings from the substrate was not observed. Details of the other wear mechanisms, such as tribochemical wear were not investigated. No transfer layer was observed on the wear track. Gogotosi et al. [178] discussed tribochemcial wear as a possible mechanism for wear in boron carbides against steel. Their studies indicated that the oxidation process in air or during the friction tests formed $\mathrm{B}_{2} \mathrm{O}_{3}$. This oxide layer on the boron carbide surface might have helped in reducing the friction.

Tables 7.5 summarizes the heats of formation of $\mathrm{B}_{4} \mathrm{C}, \mathrm{BN}$, and $\mathrm{B}_{2} \mathrm{O}_{3}$, whereas.Table 7.6 summarizes the heat of the reaction to form boron oxide.

Table 7.5 Heat of formation data for boron nitride, boron carbide, and born oxide.

\begin{tabular}{|c|c|}
\hline Compound & $\begin{array}{c}\text { Heat of formation } \\
\mathrm{kCal}^{-m o l e}\end{array}$ \\
\hline $\mathrm{B}_{4} \mathrm{C}$ & -62.7 \\
\hline $\mathrm{BN}$ & -250.9 \\
\hline $\mathrm{B}_{2} \mathrm{O}_{3}$ & -1271.9 \\
\hline
\end{tabular}


Table 7.6 Thermodynamic stability of the compound coatings.

\begin{tabular}{|c|c|}
\hline Reaction & $\begin{array}{c}\text { Heat of reaction } \\
\text { kCal.mole }\end{array}$ \\
\hline $2 \mathrm{~B}_{4} \mathrm{C}+6 \mathrm{O}_{2} \rightarrow 4 \mathrm{~B}_{2} \mathrm{O}_{3}+2 \mathrm{C}$ & -5087.6 \\
\hline $4 \mathrm{BN}+3 \mathrm{O}_{2} \rightarrow 2 \mathrm{~B}_{2} \mathrm{O}_{3}+2 \mathrm{~N}_{2}$ & -1540.2 \\
\hline
\end{tabular}

In the compound coatings that were investigated in this current study, the hardness decreased with an increase in the atomic percent of nitrogen, whereas the wear factor decreased with an increase in hardness, i.e., with an increase in the atomic percent of nitrogen. Tables 7.5 and 7.6 suggest that thermodynamically, coatings with a high atomic percent of nitrogen are susceptible to oxidization as compared to pure boron carbide.

The wear factor of uncoated silicon is greater by three orders of magnitude than that of to $\mathrm{BCN}$ coatings. This investigation on $\mathrm{BCN}$ coatings indicated high friction (0.7) with a ruby slider. As discussed in Chapter 2, the maxima of von Mises stress is located on the surface for high friction, suggesting a higher stress state, which can result in larger plastic deformation or fracture. For example, the tensile stress at the trailing edge, estimated by using Eqn.[2.16], is about $1.2 \mathrm{GPa}$. When comparing the wear behavior of the coatings, it is important to note that all five coatings have a different chemical composition. The present study revealed that abrasive wear and fracture were the mechanisms responsible for material loss in all the coatings.

Although the apparent fracture toughness of the $\mathrm{BCN}$ coating was higher than the silicon substrate, the wear morphology indicated wear by brittle fracture (Figures $7.8 \mathrm{~b}-\mathrm{c}$ ) might have been responsible for the loss of material. The current data was limited to one humidity and one dry sliding condition. The tribological properties of amorphous BCN coatings have not been investigated in the literature. This limits the direct comparison of the current results. Nastasi et al. [120] showed that nitrogen implantation into polycrystalline boron carbide reduced the friction. 
Their tests were done using a chrome steel pin. Was and Dekoven[179] found increased friction when a polycrystalline nitrogen-implanted boron carbide was rubbed against boron carbide in a vacuum. Dekempeneer et al. [177] tested BCN coatings at loads of 1 and $5 \mathrm{~N}$, with a steel pin under $50 \%$ relative humidity. Their sliding speeds were approximately the same as in this study. Their wear data indicated that the BCN coatings with greater than 50 at.\% carbon showed low friction and low wear factors relative to the silicon substrate. However, their results indicated that the coatings with intermediate compositions failed. Some of the coatings that they tested also showed high friction, as was observed in this study.

\subsection{Conclusions}

The metastable $\mathrm{BCN}$ coatings had high hardness of 20 to $40 \mathrm{GPa}$. The hardness decreased with nitrogen content in the coating. The reason for this hardness behavior is not clear at this point, but it is speculated that formation of a phase with

short range order is favored with an increase in nitrogen content in $\mathrm{BCN}$ coatings. The apparent fracture toughness of the coatings is about $30 \%$ greater than the silicon substrate. However, no significant difference in the apparent fracture toughness of different coatings was observed.

Abrasive wear seems to be the dominant wear mechanism in these coatings. Large amounts of wear debris were found in the wear track, which suggests wear by third body wear and brittle fracture of the coatings. These loose wear debris might have been responsible for the high friction against the ruby slider that was observed under the test conditions. These results are only preliminary. In order to evaluate the BCN coatings, more detailed experiments should be carried out. These experiments are discussed in the next chapter. 


\section{Chapter 8 Summary and Future ProposedWork}

The intent of this research was to broaden the application of well-established surface modification techniques and to elucidate the various wear mechanisms that occur in the sliding contact of ion-beam processed surfaces. The investigation included modifying surfaces by three surface engineering methods; namely, beam-line ion implantation, plasma-source ion implantation, and DC magnetron sputtering. The synthesized coatings were characterized for composition and microstructure, and evaluated for mechanical and tribological properties.

Surface modifications and surface coatings can alter the near-surface mechanical properties of a coating-substrate system. For example, changes in surface hardness and fracture toughness can influence the friction and wear behavior of the modified surfaces. Therefore, correlation among the surface hardness, fracture toughness, and wear behavior was also examined. The following sections summarize the three systems that were investigated in Chapters 5, 6, and 7 .

\section{Beam-Line Ion-Implantation Studies of Silicon}

Beam-line ion-implantation was used to synthesize metastable phases with unique composition and structures, i.e., exceeding equilibrium solid solubility. For example, the solubility of carbon in silicon is 50 atomic percent. For the sample implanted with a high dose of carbon, the ion beam analysis indicated 72 atomic percent of carbon was incorporated into the silicon sample.

Previous studies on carbon implantation into silicon indicated the formation of $\mathrm{Si}-\mathrm{C}$ bonds, whereas nitrogen implantation resulted in $\mathrm{N}-\mathrm{N}$ bonds. The changes in surface hardness had a correlation with the strength of the bonds that might have resulted from the implantation. For example, in the case of nitrogen implantation, when the $\mathrm{N}-\mathrm{N}$ bonds reached a critical density, nitrogen molecules were formed. 
These nitrogen molecules were trapped as gas bubbles. Thus, surface softening was observed in this sample.

The carbon-implanted samples were found to be amorphous. During the first 100 to 200 cycles of testing these samples exhibited low friction before the modified layer wore off. The reason for the low friction is speculated to be surface graphitization. The wear factor of the carbon-implanted samples was lower than the unimplanted crystalline silicon. The hardness was observed to increase with dose, and the wear factor of carbon-implanted samples also scaled with surface hardness. The improved wear behavior of the carbon-implanted samples is attributed to the presence of amorphous $\mathrm{SiC}$ and excess $\mathrm{C}$ in the modified layer.

The microstructure of nitrogen-implanted samples was also found to be amorphous. At a lower dose of nitrogen, no significant improvement was observed in the surface hardness. In contrast, at a high dose of nitrogen, the surface hardness was lower than the unimplanted silicon due to the formation of bubbles as was revealed from TEM studies. A high coefficient of friction was observed in the nitrogeninplanted samples relative to the unimplanted sample. The wear factor of the sample implanted with a low dose of nitrogen was not significantly different from the wear factor of the unimplanted sample. For the sample implanted to a high dose of nitrogen, a large number of profilometry traces were measured at different positions on the wear track. The wear area of these traces were scattered in a wide range. Thus, no conclusion could be drawn other than speculating that nitrogen bubbles in the sample might have been responsible for the scattered data.

The sample implanted with both carbon and nitrogen had a high coefficient of friction compared to the unimplanted silicon. The incorporation of nitrogen into the carbon-implanted silicon lattice was different from nitrogen incorporation into the silicon lattice. For example, carbon could have inhibited the formation of N-N bonds, as a result, surface hardness of this sample improved significantly compared to the 
unimplanted sample. However, the improvement in the wear factor for the samples implanted with both carbon and nitrogen was not as significant as the carbonimplanted samples. The wear factor value of this mixed sample was found to be the average value of the wear factor of the sample implanted at a low dose of nitrogen and a low dose of carbon. This suggested that both the carbon and nitrogen ions influenced the wear factor of the mixed implant sample.

\section{Future Proposed Work on Beam-Line Ion-Implantation Studies of Silicon}

Proposed future work should include X-ray photoelectron spectroscopy (XPS) or Auger electron spectroscopy (AES) to confirm that the chemical state of the elements in the modified layer resulted from implantation. The information obtained from these techniques would help to identify the possible phases responsible for the changes in the mechanical properties.

One should investigate carbon and nitrogen implantation at high temperatures to explore whether the crystalline phases show any changes in mechanical and tribological properties. Implantation at a high temperature can provide thermodynamic driving forces to form crystalline phases. Thus, implantation experiments at high temperature should be carried out.

Investigating the mechanical and tribological properties of the samples where nitrogen implantation is followed by carbon implantation is another possible experiment. Thermodynamically, formation of silicon carbide by implanting carbon in $\mathrm{N}$-implanted silicon seems to be unfavorable because the reaction is endothermic: $\mathrm{Si}_{3} \mathrm{~N}_{4}+3 \mathrm{C} \rightarrow \mathrm{SiC}+2 \mathrm{~N}_{2}+130 \mathrm{kcal} . \mathrm{mole}^{-1}$. Thus, the incorporation of carbon in nitrogenimplanted silicon might result in carbon-rich regions in the modified region. Depending on the nature of the carbon, i.e., whether carbon is graphitic or amorphous in form, the mechanical and tribological properties of the resulting modified layer might be different. 
One should also explore synthesis of a mixture of crystalline and amorphous structures. This can be achieved by implanting a certain atomic fraction of a species at a high temperature and then the remainder of the desired fraction at liquid nitrogen temperature. The mechanical properties of modified layers achieved by combining both hot and cold implantation could depend on the fraction of crystalline and metastable strutures. Thus, prediction of the hardness, friction, and wear behavior of these systems is complicated. However, a systematic set of experiments on these line of thoughts would provide an empirical model that can relate hardness and fraction of crystalline or amorphous phase.

Because the carbon- and nitrogen-modified layer in the present study was investigated with only one slider and at one contact stress, one should also investigate the tribological performance with other sliders and at other contact stresses. For example, the results of the tests performed with a ruby slider can be different from those that result from a metallic slider because of the difference in transfer layers and tribochemical reactions. Thermodynamic data show that the heat of reaction for the reduction of ruby with silicon is positive:

$2 \mathrm{Al}_{2} \mathrm{O}_{3}+3 \mathrm{Si} \rightarrow \mathrm{SiO}_{2}+4 \mathrm{Al}+366.36 \mathrm{kcal} \cdot \mathrm{mole}^{-1}$. Thus, one can conclude that ruby pin is chemically inert when sliding against silicon. On the other hand, a titanium pin against silicon is chemically reactive under certain testing conditions. Similarly, extending the work with a metallic slider such as nickel would produce information of interest to the micromechanical device industry that is concerned with the wear of devices.

\section{Investigations of Diamond-like Carbon Coatings}

Coatings that are applied to improve the friction and wear properties of a substrate can fail either by delamination from the substrate or by fracture within the coating. By using PSI, a graded interface between substrate and coating is formed. This interface reduces the possibility of wear by delamination. However, these 
coatings can fail by defect generation and fracture in the coating. Thus, it is advantageous to know the fracture toughness of the coatings. There are no standardized tests to measure fracture toughness of thin coatings. In this study, the fracture toughness of DLC coatings was evaluated using an indentation technique.

The DLC coatings processed by PSI, with thicknesses ranging from $0.7 \mu \mathrm{m}$ to $7 \mu \mathrm{m}$, were investigated for composition, hardness, fracture toughness, friction, and wear behavior. These DLC coatings had less than 30 atomic percent hydrogen, and the hardness of these coatings was found to be in the range of $11 \mathrm{GPa}$ to $19 \mathrm{GPa}$. No correlation was observed between the hardness and residual stress in the coatings. The coating hardness increased with coating thickness.

The fracture toughness of DLC coatings has been estimated for the first time in the literature. Based on elastic fracture, the calculated fracture toughness of the DLC coatings was found to range between 1.12 to $1.45 \mathrm{MPa} \cdot \mathrm{m}^{1 / 2}$. These values are less than diamond, but greater than silicon. The experiments provided the insight that the coating thickness, coating hardness, and residual stresses due to processing are three important parameters in evaluating the fracture toughness of a coating-substrate system. Thickness and hardness of the coating appeared to influence the initiation of the cracks, whereas the compressive stress in the film should influence crack propagation. For example, no cracks were observed at a load of $3000 \mathrm{gms}$ for a $7 \mu \mathrm{m}-$ thick DLC coating.

The values of the apparent fracture toughness of the coating-substrate system measured in this study were similar to that reported in the literature for polycrystalline diamond coatings grown on silicon substrates. The apparent fracture toughness of the coating-substrate system was also estimated at a lower limit using the Griffith model for the elastic fracture. The apparent fracture toughness determined experimentally was found to range between 0.77 and $1.52 \mathrm{MPa} . \mathrm{m}^{1 / 2}$. Values of the estimated apparent 
fracture toughness for a $1.36 \mu \mathrm{m}$ thick sample was found to be $0.82 \mathrm{MPa} \cdot \mathrm{m}^{1 / 2}$ compared to the experimentally determined value of $1.14 \mathrm{MPa} \cdot \mathrm{m}^{1 / 2}$. Thus, the experimentally determined values for apparent fracture toughness were 25 to $30 \%$ greater than the estimated values of apparent fracture toughness. Residual stresses due to processing and/or plastic deformation during indentation can influence the measured values. For example, in this study, the residual compressive stresses were found to inhibit the crack propagation. Similarly, the uncertainties associated with surface energies may affect the predicted fracture toughness values.

The data indicated a correlation between the mechanical properties, such as hardness and apparent fracture toughness, and wear factor. The DLC coatings were found to have low friction $(0.1)$ and low wear factors $\left(10^{-7} \mathrm{~mm}^{3} / \mathrm{N} . \mathrm{m}\right)$ even at the high contact stress of about $1 \mathrm{GPa}$.

\section{Future Proposed Work on Investigations of Diamond-like Carbon Coatings}

Investigations on DLC coatings revealed that the apparent fracture toughness of the coatings-substrate system is governed by coating thickness, coating hardness, and residual stresses due to processing.

More systematic experiments have to be designed in order to develop a method and an understanding towards the fracture toughness of nonuniform structures. For example, variations of coating thickness at a constant coating hardness and residual stress can help to identify the role of coating thickness on the initiation of cracks. By mechanical manipulation of substrates before deposition, processing coatings of the same thickness and hardness with varying residual stress should be explored.

Experiments on these coatings can also reveal the role of residual stress on the propagation of cracks.

When indentation experiments were performed, it was realized that the loads available to create well-defined cracks were limited because of the automation of the microindenters. Thus, the number of data points that could be obtained on a particular 
system under investigation were limited. This further adds constraints on developing any models based on experimental data. However, an older version of a microindenter can be modified to obtain additional data points. In addition, this analysis was based on the assumption that the induced cracks are half-penny shape. In reality, the shape of the cracks might deviate from the half-penny shape. Since silicon is transparent to infrared radiation, cracking in coatings on silicon substrate can be examined by infrared spectroscopy. Another approach to determine the shape of the cracks optically is by depositing the coatings on a transparent substrates such as glass.

\section{Investigations of Boron-Carbon-Nitrogen Coatings}

Boron-carbon-nitrogen coatings with varying compositions were synthesized using DC magnetron sputtering. The composition varied along the composition line between $\mathrm{B}_{4} \mathrm{C}$ and $\mathrm{N}$. The hardness of the coatings varied from 40 to $30 \mathrm{GPa}$, with an increase in nitrogen content. The measured hardness of amorphous boron carbide was $40 \mathrm{GPa}$, which is comparable to the hardness of a polycrystalline boron carbide that has been reported in the literature. The hardness decreased with increased nitrogen content, which suggested formation of a softer phase. Boron nitride exists in hexagonal and cubic structures. The reported hardness of hexagonal boron nitride is $9 \mathrm{GPa}$ and cubic boron nitride is $50 \mathrm{GPa}$. It is not clear at this point whether the metastable $\mathrm{BCN}$ coatings are composed of hexagonal or cubic boron nitride or a mixture of both forms.

\section{Future Proposed Work on Investigations of Boron-Carbon-Nitrogen Coatings}

$\mathrm{X}$-ray diffraction, TEM, Auger spectroscopy, or $\mathrm{x}$-ray photoelectron spectroscopy experiments should be carried out to obtain the information on the phases, bonding, and structure of metastable BCN compounds. Future experiments should include varying the deposition parameters to form $\mathrm{h}$-BN or any other possible phases that can act as lubricants. Post-annealing of the samples investigated in this study or synthesizing the samples with the same composition at high temperature are 
likely to result in varying proportion of two different phases. These samples are also likely to exhibit interesting mechanical and tribological properties.

The present study investigated the friction and wear behavior of metastable $\mathrm{BCN}$ coatings with a ruby slider under contact stresses ranging from 0.8 to $1 \mathrm{GPa}$. Under these test conditions, these coatings had high friction. Additional work is required to resolve whether the high friction was due to the absence of the lubricant form of h-BN or due to brittle fracture in the coatings. One would expect the h-BN phase to be more ductile and less prone to fracture. If brittle fracture was responsible, then one has to be careful in choosing hard coatings with high friction since brittle fracture may sometimes lead to the catastrophic failure of the coating.

The response of the $\mathrm{BCN}$ coatings to different testing should also be investigated. For example, the coatings should be tested with the same slider, but over a range of contact stresses to see if additional wear regimes, such as mild abrasion or plastic deformation occur. If other regimes are observed, this work could lead to the establishment of wear maps for this material system.

For practical applications, the behavior of $\mathrm{BCN}$ systems in the presence of lubricants should also be investigated. Although the high friction and brittle fracture observed in these limited unlubricated studies is somewhat discouraging, it is possible that in the presence of a lubricant, fracture may be suppressed and the benefits of the high hardness may be realized, particularly for low-angle erosion or abrasion-resistant applications.

\section{Summary}

The major contributions of this dissertation are:

- Demonstrated the ability to develop novel nonequilibrium structures using well-established processing techniques. For example, beam-line implantation was used to investigate the mixed implantation of carbon and nitrogen ions into single crystal silicon for tribological applications. 
- Initiated the research to develop and evaluate new metastable BCN coatings by $\mathrm{DC}$ magnetron sputtering for tribological applications.

- Estimated the fracture toughness of the DLC coatings using the Griffith model for elastic fracture for the first time in the literature.

- Developed a foundation for a model that could be used to determine the fracture toughness of thin coatings when the residual stresses in the coatings are known.

In summary, part of this work contributed to novel ideas in coating processes by using well-established techniques, and the other part contributes to the foundation for a potential model to measure the fracture toughness of thin coatings with known residual stresses. 


\section{REFERENCES}

1. Karl-Heinz Zum Gahr, Microstructure and Wear, Tribology Series, 10, Elsevier Science Publishers, Amsterdam, (1987).

2. N. Suh, Tribophyiscs, Prentice-Hall, New Jersey, (1986).

3. J. A. Williams, Engineering Tribology, Oxford Science Publications, New York (1994).

4. I. M. Hutchings, Tribology, CRC Press, Florida (1992).

5. E. Rabinowicz, Friction and Wear of Materials, John Wiley \& Sons, New York (1995).

6. J. Halling, Principles of Tribology, Maxmillan Press, London (1978).

7. R. D. Arnell, P. B. Davies, J. Halling, and T. L. Whomes, Tribolgy, SpringerVerlag, New York (1993).

8. C. Lim and M. F. Asby, "Wear-Mechanism Maps," Acta. Metall. 35(1):1-24 (1987).

9. J. Krim, "Friction at the Atomic Scale," Scientific American, p.74 (October 1996).

10. M. Hsu, P. I. Lacey, Y. S. Wang, and S. W. Lee, "Wear Mechanism Maps of Ceramics," Advances in Engineering Tribology, Eds. Y. Chung and H. S. Chung, Society of Tribology and Lubrication Engineering, SP-31:123-131, (1991).

11. S. W. Lee, S. M. Hsu, and M. C. Shen, "Ceramic Wear Maps," Zirconia, J. Amer, Ceram. Soc. 76(8):1937-1947 (1993).

12. Y. S. Wang, S .M. Hsu, and R. G. Munro, "Ceramic Wear Maps: SiC Whisker Reinforced Alumina," J. Society of Tribolgy and Lubrication Engineers 47(1): 63-69 (1990).

13. Y. S. Wang and S. M. Hsu, "Wear and Wear Transition Modeling of Ceramics," Wear 195(1-2):112-122 (1996).

14. S. W. Lee, M. Shen, and S. M. Hsu, "Ceramic Wear Maps: Silicon Nitride," Key Engineering Materials 89-9:751-755 (1994).

15. S. Wang and S. M. Hsu, "Wear and Wear Transition Modeling of Ceramics," Wear 195(1-2):35-46 (1996).

16. M. Nastasi, J. W. Mayer, and J. K. Hirvonen, Ion-solid Interactions, Cambridge University Press, New York (1996).

17. W. C. Oliver, R. Hutchings, and J. B. Pethica, "The Wear Behaviour of Nitrogen Implanted Metals," Metall. Trans. 15A:2221-2229 (1984). 
18. R. Hutchings and W. C. Oliver, "A Study of the Improved Wear Performance of Nitrogen Implanted Ti6A14V," Wear 92:143-153 (1983).

19. T. Hoike, A. Itoh, S. Noda, H. Doi, J. Kawamoto, and J. Kamigaito, "Strengthening of $\mathrm{Al}_{2} \mathrm{O}_{3}$ and Ion Implantation," J. Mater. Sci. Lett. 3:1099-1101 (1981).

20. S. Eskildsen and G. Sorensen, "On the Use of Reactive Ion Beam Mixing for Surface Modifications," Surface Engineering, pp. 48-60, Martinus Nijhoff Publishers, Dordrecht, Netherlands (1984).

21. G. Dearnaley, "Improvement of Wear Resistance in Cemented Tungsten Carbide by Ion Implantation," Proc. Int. Conf. on Science of Hard Materials, pp. 467-484 (1983).

22. M. Wantanabe, H. Shimura, and Y. Enomoto, "Effect of Nitrogen Ion Implantation on the Friction and Wear Properties of Some Plastics," Proceedings of 16th Leeds-Lyon Symposium on Tribology, Elsevier Science Publishers, Amsterdam (1989).

23. J. Lekki, Z. Statchura, N. Preikschas, B. Cleff, M. Cholewa, and G. J. F. Legge, "Friction and Wear of Argon Implanted Silicon Crystals," J. Mater. Res. 9(1):9195 (1994).

24. J. Burnett and T. F. Page, "Surface Softening in Silicon by Ion Implantation," J. Mater. Res. 9(1): 91-96 (1984).

25. T. Miyamoto, S. Miyake and R. Kaneko, "Wear resistance of C+ Implanted Silicon Investigated by scanning Probe Microscopy," Wear. 162-164:733-738 (1993).

26. B. K. Gupta, J. Chevallier, and B. Bhushan, "Tribology of Ion Bombarded Silicon for Micromechanical Applications," Transaction of ASME 115: 392-399 (1993).

27. R. Conrad, "Methods and Apparatus for Plasma Source Ion Implantation," US patent 4,764,394 (1988).

28. R. Conard, R. A. Dodd, and F. J. Worzala and X. Qiu, "Plasma Source Ion Implantation: A New, cost-effective, Non-Line-Of-Sight Technique for Ion Implantation of Materials," Surf. Coat. Technol, 36:927-937 (1988).

29. R. Conard R. A. Dodd, S. Han, M. Madapura, J. Scheuer, K. Sridharan, and F. J. Worzala, "Manufacturing Aspects of Plasma Source Ion Implantation," ASME, High Energy Beam Manufacturing Technologies, 41 :65-70 (1989).

30. R. Conard, "Plasma Source Ion Implantation: A New Approach to Ion Beam Modification of Materials," Material Science and Engineering, A116:197-203 (1989).

31. R. Conard, J. L. Radtke, R. A. Dodd, F. J. Worzala, and N. C. Tran, "Plasma Source Ion Implantation Technique for Surface Modification of Materials," Appl. Phys. 62:4591-4596 (1987). 
32. K. C. Walter, M. Nastasi, H. Kung, P. Kodali, C. Munson, I. Henins, and B. P. Wood, "Diamond-Like Carbon Deposition for Tribological Applications at Los Alamos National Laboratory," Mat. Res. Soc. Symp, Proc., 383:411-422 (1995).

33. M. Samandi, A. Pauza, G. Hatziamdoniou, H. Yasbandha, R. Hutchings, G.A. Collins, and J. Tendys, "Microstructure and Tribological Behavior of Plasma Immersion Ion-Implanted Tool Steels," Surf. Coat. Technol, 54/55:447-452 (1992).

34. M. Samandi, B. A. Shedden, D. J. Smith, G. A. Collins, R. Hutchings, and J. Tendys, "Microstructure, Corrosion, and Tribological Behavior of Plasma Immersion Ion-Implanted Austenitic Stainless-Steel," Surf. Coat. Technol. 59:261-265 (1993).

35. M. Redsten, K. Sridharan, F. J. Worzala, and J. R. Conrad, "Nitrogen Plasma Source Ion-Implantation of AISI S1 Tool Steel," J. Mater. Process. Technol. 30:253 (1992).

36. G. A. Collins, R. Hutchings, and J. Tendys, "Plasma Immersion Ion-Implantation of Steels," Mat. Sci. Eng. A 139:171-178(1991).

37. J. V. Mantase, I. G. Brown, N. W. Cheung, and G. A. Collins, "Plasma Immersion Ion-Implantation," Mat. Res. Soc. Bull., 52-56 (August, 1996).

38. K. C. Walter, "Nitrogen Plasma Source Immersion Ion-Implantation of Aluminum," J. Vac. Sci. Technol. B12:945-950 (1994).

39. J. N. Matosssian, J. J. Vajo, J. A. Wysocki, and M. E. Bellon, "Plasma Immersion Ion-Implantation (PII) to Improve the Wear Life of Tungsten Carbide Drill Bits Used in Printed Wiring Board (PWB) Fabrication," Surf. Coat. Technnol. 62:595599 (1993).

40. L. Xie, F. J. Worzala, J. R. Conrad, and R. A. Dodd, "Influence of Temperature on Nitrogen Ion Implantation of Incology Alloys 908 and 909," Mater. Sci. Eng. A139:179-184 (1991).

41. N. C. Horswill, K. Sridharan, and J. R. Conrad, "A Fretting Wear Study of Nitrogen-Implanted Titanium-Alloy,” Mater. Sci. Lett. 14:1349-1351 (1995).

42. K. C. Walter, J. T. Scheuer, P. C. McIntyre, P. Kodali, N. Yu, M. Nastasi, "Increased Wear-Resistance of Electrodeposited Chromium through Applications of Plasma Source Immersion Ion-Implantation Techniques," Surf. Coat. Technol. 85(1): 1-6 (1996).

43. L. A.Thomson, F. C. Law, N. Ruchton, and J. Franks, "Biocompatability of Diamond-Like Carbon Coating," Biomaterials 12:37-40 (1991).

44. J. P. Hirvonen, R. Lappalainen, J. Koskinen, A. Antilla, T. R. Jervis, and M. Trkula, "Tribological Characteristics of Diamond-Like Carbon Films Deposited with an Arc-Discharge Method," J. Mater. Res. 5:2524-2530 (1990).

45. K. Enke, H. Dimigen, and H. Hubsch, "Frictional Properties of Diamond-Like Carbon Layers,” Appl. Phys. Lett. 36:291-292 (1980). 
46. R. Wei, P. J. Wilbur, A. Erdemir, and F. M. Kustas, "The Effects of Beam Energy and Substrate Temperature on the Tribological Properties of Hard-Carbon Films on Aluminium," Surf. Coat. Technol. 51:139-145 (1992).

47. R. L. C. Wu, "Synthesis and Characterization of Diamond-Like Carbon Films for Optical and Mechanical Properties," Surf. Coat. Technol. 57:258-266 (1992).

48. A. Itoh, S. Hibi, T. Hioki, and J. Kawamoto, "Tribological Properties of Metals Modified by Ion-Beam Assisted Deposition of Silicone Ion," J. Mater. Res. 6(4):871-874 (1991).

49. B. Andre, J. P. Nabot, L. Lombard, P. Martin, "From Amorphous Carbon to Diamond-Like Carbon," NATO ASI Series E, 266:313-316 (1991).

50. K. C. Walter, H. Kung, T. Levine, J. T. Tesmer, P. Kodali, B. P. Wood, D. J. Rej, M. Nastasi, J. Koskinen, and J. P. Hirvonen, "Characterization and Performance of Carbon Films Deposited by Plasma and Ion Beam Based Techniques," Mat. Res. Soc. Symp. Proc. 354:87-92 (1995).

51. K. C. Walter, H. Kung, T. Levine, J .T. Tesmer, P. Kodali, B. P. Wood, D. J. Rej, M. Nastasi, J. Koskinen, and J. P. Hirvonen, "Characterization and Performance of Diamond-Like Carbon Films Synthesized by Plasma- and Ion-Beam-Based Techniques," Surf. Coat. Technol. 74-75:734-738 (1995).

52. M. Tamor, "Synthesis, Properties and Applications of Superhard Amorphous Coatings," Applications of Diamond Coatings and Related Materials, Eds. A. Feldman, Y. Tzeng, W. A. Yarbrough, M. Yoshikawa, and M. Muruakawa, pp. 691-702 (1995).

53. M. Nastasi, A. A. Elmoursi, R. J. Faehl, A. H. Hamdi, I. Henins, G. W. Malaczynski, J. V. Mantese, C. Munson, X. Qui, W. A. Reass, D. J. Rej, J. T Scheuer, C. E. Speck, K. C. Walter, and B. P. Wood, "Material Issues in Plasma Source Ion Implantation," Mat. Res. Soc. Symp. Proc. 396:455 (1995).

54. L. J. McColm, Ceramic Hardness, Plenum Publishing Corporation, New York (1986).

55. K. Holmberg, A. Mathews, "Coatings Tribology: Properties, Techniques and Applications," Surface Engineering, Ed. D. Dowson, Elsevier Science Publishers, Amsterdam, 28:214-215 (1994).

56. H. A. Sundquist, E. H. Sirvio, and T. M. Kurkinen, "Wear of Metal-Working Tools Ion-Plated with Titanium Nitride," Metals Technology 10:130-134(1983).

57. J. Franks, K. Enke, and A. Richardt, "Diamond-Like Carbon--Properties and Applications," Metals and Materials, pp. 695-700 (Nov. 1990).

58. J. A. Greenwood and J. B. P. Williamson, "Contact of Nominally Flat Surfaces," Proc. R. Soc. Lond., A295:300-319 (1966).

59. F. P. Bowden, P. Moore, and D. Tabor, "The Ploughing and Adhesion of Sliding Metals," J. Appl. Phys. 14:80-91 (1943). 
60. F. P. Bowden and D. Tabor, The Friction and Lubrication of Solids, Part I, Clarendon Press, Oxford (1950).

61. A. P. Green, "Plastic Yielding of Metal Junctions Due to Combined Shear and Pressure," J. Mechanics and Physics of Solids 2:197-211 (1955).

62. A. P. Green, "Friction Between Unlubricated Metals," Proc. R. Soc. Lond. A228:191-204 (1955).

63. R. T. Spurr, "The Role of Adhesion in Friction, Friction and Traction," Proceedings of 7th Leeds-Lyon Symposium on Tribology, edited by D. Dowson, C. M. Taylor, M. Godet, and D. Berthe, Westbury House, London, pp. 34-38 (1981).

64. K. L. Johnson, "Aspects of Friction, Friction and Traction," Proceedings of 7th Leeds-Lyon Symposium on Tribology, edited by D. Dowson, C. M. Taylor, M. Godet, and D. Berthe, Westbury House, London, pp. 34-38 (1981), pp. 3-12 (1981).

65. D. H. Buckley, Surface Effects in Adhesion, Friction, Wear, and Lubrication, 315-427, Elsevier Science Publishers, Amsterdam (1981).

66. S. T. Okaty and N. P. Suh, "WearDebris Formation and Agglomeration," J. Tribology, Transaction of ASME 114:379-393 (1992).

67. J. M. Challen and P. L. B. Oxley, "An Explanation of Different Regimes of Friction and Wear Using Asperity Deformation Models," Wear 53:229-243 (1979).

68. J. P. Hirth and D. A. Rigney, "Crystal Plasticity and the Delamination Theory of Wear," Wear 39:133-141 (1976).

69. D. A. Rigney and J. P. Hirth, "Plastic Deformation and Sliding Friction of Metals,"Wear 53:345-370 (1979).

70. D. A. Hills and D. W. Ashelby, "On the Application of Fracture Mechanics to Wear," Wear 54:321-330 (1979).

71. Fundamentals of Friction, Eds. I. R Singer and H. M. Pollock, Kluwer Academic Publisheres, Dordrecht, Netherlands (1992).

72. B. Bhushan, J. N. Israelachvili, and U. Landman, "Nanotribology: Friction, Wear and Lubrication at the Atomic Scale," Nature 374:607-616(1995).

73. Handbook on Micro/Nanotribology, Ed. Bharat Bhushan, CRC Press, Florida (1995).

74. T. E. Fischer and H. Tomizawa, "Interaction of Tribochemistry and Microfracture in the Friction and Wear of Silicon Nitride," Wear 105:29-45 (1985).

75. J. F. Archards, "Elastic Deformation and Laws of Friction," Proc. R. Soc. A, 243:190-205 (1957). 
76. J. F. Archards, "Contact and Rubbing of Flat Surfaces," J. Appl. Phys. 24:981988 (1953).

77. N. P. Suh, "The Delamination Theory of Wear," Wear 25:111-124 (1973).

78. S. Jahanmir and N. P. Suh, Mechanics of Subsurface Void Nucleation in Delamination Wear, Wear 44:17-38 (1977).

79. A. G. Atkins, "Fracture Toughness and Cutting," Int. J. Prod. Res. 12:263-274 (1974).

80. E. Hornbogen, "The Role of Fracture Toughness in the Wear of Metals," Wear 33:251-259 (1975).

81. M. A. Moore and F. S. King, "Abrasive Wear of Brittle Solids," Wear 60:123-140 (1980).

82. A. R. Rosenfield, "A Fracture Mechanics Approach to Wear," Wear 61:125-132 (1980).

83. A. G. Evans, Abrasive Wear in Ceramics, Science Ceramic Machining and Surface Finishing II, National Bureau of Standards Special Publication 562, pp.1-14 (1979).

84. S. M. Hsu, K. Klaus, and H. S. Cheng, "A Mechano-Chemical Descriptive Model For Wear Under Mixed Lubrication," Wear 128:307-323 (1988).

85. J. Halling, “A Contribution to Theory of Mechanical Wear," Wear 34:239-249 (1975).

86. K. Kato, "Micro-Mechanics of Wear-Wear Modes," Wear 153:277-295 (1992).

87. H. Hertz, "Miscellaneous Papers by Hertz," Eds. Jones and Schott, Macmillan, London (1896).

88. K. L. Johnson, Contact Mechanics, Cambridge University Press, New York (1985).

89. G. E. Dieter, Mechanical Metallugy, 3rd. edn., McGraw-Hill, New York (1986).

90. G. M. Hamilton and L. E.Goodman, "The Stress Field Created by a Circular Sliding Contact," J. Applied Mechanics 33:371-376 (1966).

91. G. M. Hamilton, "Explicit Equations for the Stresses Beneath a Sliding Spherical Contact," Proc. Inst. Mech. Eng. 197c:53-59(1983).

92. D. M. Burmister, "The General Theory of Stresses and Displacements in Layered Systems," J. Appl. Physics, 16:89-94 (1945).

93. W. T. Chen, "Computation of Stresses and Displacements in a Layered Elastic Medium," Int. J. of Eng. Science 9:775-799 (1971).

94. P. K. Gupta and J. A. Walowait, "Contact Stress between an Elastic Cylinder and Layered Elastic Solid," J. Lubr. Technon. 96:250-257 (1974). 
95. S.Van Der Zwagg and J. E. Field, "The Effect of Thin Hard Coatings on the Hertzian Stress Field," Phil. Mag. A 46:133-150 (1982).

96. H. Djabella and R. D. Arnell, Finite Element Analysis of the Contact Stresses in Elastic Coating on an Elastic Substrate, Thin Solid Films 213:205-219(1992).

97. K. Komvopolus, "Finite Element Analysis of a Layered Elastic Solid in Normal Contact with a Rigid Surface," J. of Tribology 110:477-484 (1988).

98. R. B. King and T. C. O'Sullivan, "Sliding Contact Stresses in a Two-Dimensional Layered Elastic Half-Space," Int. J. Solids and Structures 23(5):581-595 (1987).

99. T. C. O'Sullivan and R. B. King, "Sliding Contact Stress Field Due to a Spherical Indenter on a Layered Elastic Half-Space," J. of Tribology 110:235-240 (1988).

100. H. Djabella and R. D. Arnell, "Finite Element Analysis of the Contact Stresses in Elastic Coating/Substrate under Normal and Tangential Load," Thin Solid Films 223:87-97 (1993).

101. J. M. Leroy and B. Villechaise, "Stress Determination in Elastic Coatings and Substrate Under Both Normal and Tangential Loads," Tribolgy Series 17, Leed's-Lyon Symp., pp.195-201 (1989).

102. H. Djabella and R. D. Arnell, "Finite Element Comparative Study of Elastic Stresses in Single, Double Layer, and Multilayered Coated Systems," Thin Solid Films 235:156-162 (1993).

103. H. Djabella and R. D. Arnell, "Finite Element Analysis of Elastic Stresses in Multilayered Systems," Thin Solid Films 245:27-33 (1994).

104. K. G. Budinski, Surface Engineering for Wear Resistance, Prentice-Hall, New Jersey (1988).

105. J. F. Zeigler, J. P. Biersack, and U. Liimark, The Stopping Range of Ions in Solids, Pergmon Press, New York (1985).

106. J. Lindahrd, M. Scharff, and H. E. Schiott, "Range Concepts in Hevay Ion Ranges," Mat. Fys. Medd. Dan Vid Selsk., 33(14):3-40 (1963).

107. J. W. Mayer, L. Eriksson, and J. A. Davies, Ion Implantation in Semiconductors, Academic Press, New York (1970).

108. K. B. Winterborn, P. Sigmund, and J. B. Sanders, "Spatial Distribution of Energy Deposited by Atomic Particles in Elastic Collisions," Mat. Fys. Medd. Dan Vid Selsk. 37(14):5-40 (1970).

109. G. Dearnaley, "Applications of Ion-Implantation in Metals," Thin Solid Films 107(3):315-326 (1983).

110. H. Dimigen, K. Kobs, R. Leutenecker, H. Ryssel, and P. Eichinger, "Wear Resistance of Nitrogen Implanted Steels," Mater.Sci. Eng. 69:181-190(1985). 
111. D. M. Follstaedt, L. E. Pope, J. A. Knapp, S. T. Picraux, and F. G. Yust, "The Microstructure of Type-304 Stainless-Steel Implanted with Titanium and Carbon and its Relation to Friction and Wear Tests," Thin Solid Films 107(3):259-267 (1983).

112. N. E. W. Hartley, "The Tribology of Ion Implanted Metal Surfaces," Tribol. Int. 8:65-72 (1975).

113. N. E. W. Hartley, "Ion Implantation and Surface Modification in Tribology," Wear 34:427-438 (1975).

114. G. K. Hubler and F. A. Smidt, "Application of Implantation to Wear Protection of Materials," Nucl. Instrum. Meth. Phys. Res. B7/8:151-157(1985).

115. A. Itoh, T. Hioki, and J. Kawamoto, "Tribological Properties of Ion Implanted Sic Ceramics," Nucl. Instrum. Meth. Res. B37/38:692-695 (1989).

116. M. Iwaki, "Surface Layer Characteristics of Ion Implanted Metals," Thin Solid Films 101:223-231 (1983).

117. W. R. Jones Jr. and J. Ferrante, "Tribological Characterstics of Nitrogen $\left(\mathrm{N}^{+}\right)$ Implanted Iron," ASLE Trans. 26:351-359 (1983).

118. A. Kluge, K. Langguth, R. Ochsner, K. Kobs, and H. Ryssel, "A Comparison of the Wear Behavior of Ag, B, C, N, Pb and Sn Implanted Steels with 1.5-percent to 28 percent Chromium," Nucl. Instrum. Meth. Phys. Res. B39:531-534(1989).

119. P. B. Madakson, "Mechanical Properties of Nitrogen Implanted $18 \mathrm{~W}-4$ Cr-1V Bearing Steel," Mater. Sci. Eng. 90:287-290(1987).

120. M. Nastasi, R. Kossowsky, J. P. Hirvonen, and N. Elliott, "Friction and Wear Studies in $\mathrm{N}$-implanted $\mathrm{Al}_{2} \mathrm{O}_{3}, \mathrm{SiC}, \mathrm{TiB}_{2}$, and $\mathrm{B}_{4} \mathrm{C}$ Ceramics," J. Mater. Res. 3(6):1127-1133 (1989).

121. I. Singer, R. N. Bolster, and C. A. Carosella, "Abrasive Wear Resistance of Titanium- and Nitrogen-Implanted 52100 Steel Surfaces," Thin Solid Films 73:283-289 (1980).

122. A. K. Suri, R. Nimmagadda, and R. F. Bunshah, "Influence of Ion-Implantation and Overlay Coatings on Various Physico-Mechanical and Wear Properties of Stainless-Steel, Titanium and Aluminum," Thin Solid Films 64(2):191-203 (1979).

123. J. Chen, J. R. Conrad, and R. A. Dodd, "Structure and Properties of Amorphous Diamond-Like Carbon Films Produced by Ion-Beam Assisted Plasma Deposition," J. Mat. Engin. Perform. 2:839-843 (1993).

124. Handbook of Modern Ion Beam Materials Analysis, Eds. J. T. Tesmer and M. Nastasi, Materials Research Society, Pittsburgh (1995).

125. L. R. Doolittle, "Algorithms for Rapid Simulation of Rutherford Backscattering Spectra," Nucl. Instr. Methods. B9:344-351 (1985). 
126. C.A. Volkert, "Stress and Plastic Flow in Silicon During Amorphization by Ion Bombardment," J. App. Phyiscs 70:3521-3527 (1991).

127. M. F. Doerner and W. D. Nix, "A Method of Interpreting the Data from the Depth Sensing Indentation Instruments," J. Mat. Res. 1(4):601-609 (1986).

128. S. J. Zinkle and J. S. Huang, "Mechanical Properties of Carbon-Implanted Niobium," Mat. Res. Soc. Symp. Proc. 188:121-128 (1989).

129. T. E. Schlesinger, R. C. Camarata, C. Kim, S. B. Qadri, and A. S. Edelstein, "Mechanical Properties of Multilayered Copper-Nickel Thin Films Measured by Indentation Techniques," Mat. Res. Soc. Symp. Proc. 188:295-300 (1989).

130. G. R. Anstis, P. Chantikul, D. B. Marshall, and B. R. Lawn, A Critical "Evaluation of Indentation Techniques for Measuring Fracture Toughness: I. Direct Cracks Measurements, II. Strength Method," J. Amer. Ceram. Soc. 64(9):533-538 (1981).

131. W. D. Kingry, H. K. Brown, and D. R. Uhlmann, Introduction to Ceramics, John Wiley \& Sons, New York (1976).

132. R. G. Rhodes, Imperfections and Active Centers in Semiconductors, MacMillan, New York (1964).

133. R. C. Newman and J. B. Willis, "Vibrational Absorption of Carbon in Silicon," J. Phys. Chem. Solids 26:373-379 (1965).

134. R. C. Newman and J. Wakefield, "The Diffusivity of Carbon in Silicon," J. Phys. Chem. Solids 19:230-234 (1961).

135. F. Shiumura, Semiconductor Silicon Crystal Technology, Academic Press, San Diego (1989).

136. A. M. Brown and M. F. Ashby, "Correlations for Diffusion Constants," Acta Metallrgica 28:1085-1101 (1980).

137. K. Kijima and S. Shirasaki, "Nitrogen Self-Diffusion in Silicon Nitride," $J$. Chem. Phys. 65(7):2668-2671 (1976).

138. B. Cantor, "Diffusion in Amorphous Alloys," Rapidly Quenched Metals, Proceedings of Fifth International Conference, edited by S. Steeb and H. Warlimont, Vol. 1:595-602 (1985).

139. K. Nordlund and R. S. Averback, "Atomic Displacement Processes in Irradiated Amorphous and Crystalline Silicon," Appl. Phys. Lett., accepted.

140. H. J. Stein, "Nitrogen in Crystalline Si," Mater. Res. Soc. Sym. Proc. 59:523-535 (1986).

141. T. Abe, H. Harada, N. Ozawa, and K. Adomi, “Deep Level GenerationAnnihilation in N-doped FZ Crystals," Mater. Res. Soc. Sym. Proc., 59:537-551 (1986). 
142. R. J. Dexter, S. B. Watelski, and S. T. Picrauz, "Epitaxial Silicon Layers Grown on Ion-Implanted Silicon Nitride Layers," Appl. Phys. Lett. 23:455-458 (1973).

143. T. Kimura, S. Kagiyama, and S. Yugo, "Structure and Annealing Properties of Silicon Carbide Thin Layers Formed by Implantation of Carbon Ions in Silicon," Thin Solid Films 81:319-327 (1981).

144. T. Kimura, S. Kagiyama, and S. Yugo, "Characteristics of the Synthesis of $\beta-$ SiC by the Implantation of Carbon Ions into Silicon," Thin Solid Films 94:192198 (1982).

145. O. Kubaschewski and C. B. Alcock, Metallurgical Thermochemistry, 5th edition, Pergamon, Oxford (1979).

146. J. Dean, Lange's Handbook of Chemistry, 14th Edition, McGraw-Hill, New York (1976).

147. J. E. Field, Propeties of Diamond, Academic Press, New York (1979).

148. C. R. Brookes, "Scratch and Indentation of Crystals," Phil. Mag. 43(3):529-543 (1981).

149. A. Kelly and N. H. Macmillan, Strong Solids, 3rd Edition, Clarendon Press, Oxford Press, New York (1986).

150. H. J. Mcskimin, P. Andereatch, and P. Glynn, "Elastic Stiffness Moduli of Diamond," J. Appl. Phys. 43:985-987 (1972).

151. X. Jiang, J. W. Zou, K. Reichelt, P. Grunberg, "The Study of Mechanical Properties of A-C-H Films by Brillouin-Scattering and Ultra Load Indentation," J. Appl. Phys. 66(10):4729-4735 (1989).

152. X. Jiang, J. W. Zou, B. Stritzker, "The Hardness and Young Modulus of Amorphous Hydrogenated Carbon and Silicon Films Measured with an Ultralow Load Indenter," J. Appl. Phys. 66(12):5805-5808 (1989).

153. N. H. Cho, K. M. Krishnan, D. K. Veirs, M. D. Rubin, C. B. Hopper, B. Bhushan, and B. G. Bogy, "Chemical Structure and Physical Properties of Diamond-Like Amorphous Carbon Films Prepared by Magnetron Sputtering," J. Mater, Res. 5(11):2543-2554 (1990).

154. P. J. Martin, S. W. Fillpczuk, R. P. Netterfield, J. S. Field, D. F. Whitall, and D. R. Mckenzie, "Structure and Hardness of Diamond-Like Carbon Films Prepared by Arc Evaporation," J. Mat. Sci. Lett. 7:410-412 (1988).

155. K. Koskinen, "Abarsive Wear Resistance of Ion-Deposited Hard Carbon Films as a Function of Deposition Energy," J. Appl. Phys. 63(6):2094-2097 (1988).

156. J. X. Zhao, R. C. Bradt, P. L. Walker, "Effect of Air Oxidation at $873-\mathrm{K}$ on the Mechanical Properties of a Carbon Carbon Composite," Carbon 23(1):9-13 (1985). 
157. H. M. Hawthrone, The Microindentation of Hardness Behavior of Carbon Filaments, Glassy Carbons, And Pyrolytic Graphites," Carbon 13:215-233 (1975).

158. X. Jiang, J. W. Zou, B. Stritzker, "Mechanical Properties of a-C:H Films Prepared by Plasma Decomposition of $\mathrm{C}_{2} \mathrm{H}_{2}$," J. Appl. Phys. 68:1018-1022 (1990).

159. J. J. Mecholsky, Y. L. Tsai, and W. R. Drawl, "Fracture Studies of Diamond Films on Silicon," J. Appl. Phys. 71:4875-4881 (1992).

160. A. A. Griffith, The Phenomena of Rupture and Flow of Solids," Phil. Trans. Roy. Soc. Lond A221:163-198 (1921).

161. J. J. Gilman, "Direct Measurements of the Surface Energies of Crystals," J. App. Phys. 31:2208-2218 (1960).

162. R. J. Jaccodine, "Surface Energy of Germanium and Silicon," J. Electroche. Soc. 110:524-527 (1963).

163. J. E. Field and C. J. Freeman, "Strength and Fracture Properties of Diamond," Phil. Mag. 43:595-618 (1981).

164. S. Ramaseshan, "The Cleavage Properties of Diamond," Proc. Ind. Aca. Sci. A24:114-121 (1946).

165. E. Orowan, "Fracture and Strength of Solids," Rep. Prog. Phy. 12:185-232 (1949).

166. M. Polyani, Uber die Natur des Zerreibvorganges, Z. Phys. 7:323-327 (1921).

167. B. Lawn, Fracture of Brittle Solids, Cambridge University Press, New York (1995).

168. S. M. Ohja, H. Nostrom, and D. Mcculch, "The Growth Kinetics and Properties of Hard and Insulating Carbonaceous Films Grown," Thin Solid Films 60:213225 (1979).

169. R. L. Wu, K. Miyoshi, R. Vuppuladhadium, and H. E. Jackson, "Physical and Tribological Properties of Rapid Thermal Annealed Diamond-Like Carbon Films," Surf. Coat. Technol. 54/55:576-580 (1992).

170. R. Wei, and P. J. Wilbur, M-J. Liston, and G. Lux, "Rolling-Contact-Fatigue Wear Characteristics of Diamond-Like Hydrocarbon Coatings on Steel,"Wear 162-164:558 (1993).

171. A. Erdemir, M. Switala, R. Wei, and P. J. Wilbur, “A Tribological Investigation of the Graphite To Diamond-Like Behavior of Amorphous Carbon Films Deposited on Ceramic Substrates," Surf. Coat. Technol., 50:17-23 (1991).

172. Y. Liu, A. Erdemir, E. Meletis, "An Investigation of the Relationship Between Graphitization and Frictional Behavior of DLC Coatings," Surf. Coat. Technol. 82:48-56 (1996). 
173. J. Robertson, "Properties of Diamond-Like Carbon," Surf. Coat. Technol. 50:185-203 (1992).

174. R. D. Arnell, "The Mechanisms of Tribology of Thin Film Systems," Surf. Coat. Technol. 43/44:674-687 (1990).

175. T. M. Besman, "Chemical Vapor Deposition in the Boron-Carbon-Nitroegn System," J. Am. Ceramic. Soc. 73(8):2498-2501 (1990).

176. B. M. Devkovven, P. L. Hagans, and J. Leddy And, K. Kar, "Formation of a BN Layer on the Surface of B4C Using Nitrogen Implantation," Surf. Coat. Tech. 36:207-218 (1988).

177. E. H. A. Dekemppeneer, J. Meneve, S. Kuyers, and J. Smeets, "Tribological Properties of r.f. PACVD Amorphous B_N_C Coatings," Thin Solid Films 281/ 282:331-333 (1996).

178. Yu. G. Gogotsi, A. M. Koval'chenko, and I. A. Kossoko, "Tribochemical Interactions of Boron Carbides Against Steel," Wear 154:133-140 (1992).

179. G. Was and B. M. Dekoven, "Hardness and Friction of $\mathrm{N}_{2}^{+}$and $\mathrm{Al}^{+}$Implanted $\mathrm{B}_{4} \mathrm{C}$," Nucl. Instrum. Meth. Res. B59/60:802-805 (1991).

180. R. W. Armstrong, A. W. Ruff, H. Shin, "Elastic, Plastic, and Cracking Indentation Behavior of Silicon Crystals," Material Science and Engineering, A209:91-96(1996). 